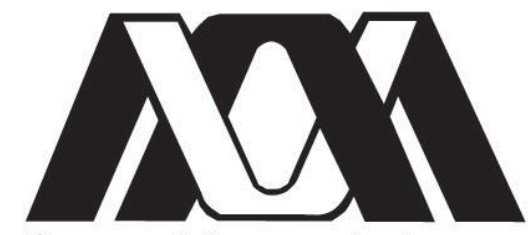

Casa abierta al tiempo

UNIVERSIDAD AUTÓNOMA METROPOLITANA
UNIVERSIDAD AUTÓNOMA METROPOLITANA UNIDAD

IZTAPALAPA

POSGRADO DE ENERGÍA Y MEDIO AMBIENTE

\title{
DISEÑO Y CARACTERIZACIÓN DE UN SISTEMA DE MICRO-COGENERACIÓN CON TURBINA DE GAS Y RECUPERACIÓN DE CALOR PARA GENERACIÓN DE VAPOR
}

\section{TESIS}

Que para obtener el grado de

MAESTRO EN CIENCIAS (ENERGÍA Y MEDIO AMBIENTE)

PRESENTA:

Ing. Adriana Santamaria Padilla

Director:

Dr. Hernando Romero Paredes Rubio 


\section{AGRADECIMIENTOS}

La Maestría en Ciencias (Energía y Medio Ambiente) de la Universidad Autónoma Metropolitana Unidad Iztapalapa, pertenece al Padrón Nacional de Posgrados de Calidad del CONACyT y cuenta con apoyo del mismo Consejo con el convenio 003893; por lo que agradece la beca recibida, a través del convenio No. 814358 para el desarrollo de esta tesis.

Esta tesis fue desarrollada con el apoyo parcial recibido a través del Centro Mexicano de Innovación en Energía Solar (CeMIE-Sol), en el marco de la Convocatoria 2013-02, del Fondo SECTORIAL CONACYT-SENERSUSTENTABILIDAD ENERGÉTICA, dentro del Proyecto Estratégico No. 10, con título: “COMBUSTIBLES SOLARES Y PROCESOS INDUSTRIALES”, por medio del cual fue posible desarrollar la investigación. 


\section{AGRADECIMIENTOS PERSONALES}

"Hay una fuerza motriz más poderosa que el vapor, la electricidad y la energía atómica:

la voluntad"

-Albert Einstein-

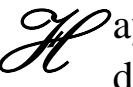

ay una cosa llamada tiempo, es una magnitud física elemental, con la cual podemos medir la duración de las cosas. Se puede medir la duración de las personas en nuestra vida. Cada persona de alguna u otra manera aporta su granito de arena para formar la persona que hoy en día somos, en este caso, de quien soy yo. Hay quienes llegaron como estrellas fugaces, y aunque corta su estancia me dejaron muchas enseñanzas. También hay quienes llegaron para quedarse un tiempo más prolongando y me acompañan a cada paso que doy, algunos un poco en la distancia y otros tan cerca que a veces no me doy cuenta de que ahí están.

En la forma que estén no importa, lo importante es que me han apoyado a lo largo de este camino, el cual es una carretera sin fin. No sé con exactitud a donde voy a llegar, pero si puedo elegir con quien recorrer ese camino. Habrá veces que el camino parezca angosto, pero siempre habrá espacio para que alguien me acompañe. La compañía nos hace ser mejores personas. Compartir nuestros logros y fracasos nos sirve para ser mejores cada día. Siempre hay algo nuevo que aprender, si nos caemos nos levantamos y si no podemos por nosotros mismos para eso están la familia y los amigos; para extender su mano cuando lo necesitamos. Este trabajo no hubiera sido posible sin ustedes.

Razón por la cual no necesito hacer alguna dedicatoria para cada uno de ustedes, nunca acabaría, y cada uno sabe que aprecio y valoro el tiempo compartido conmigo. Mi gratitud hacia ustedes es infinita y amo todos los momentos compartidos: alegrías, tristezas, frustraciones y miedos.

No siempre sabemos lo que tenemos hasta que lo perdemos, el tiempo a veces también nos separa de personas que amamos, a mis amigos y familiares que he perdido en el camino (físicamente) también les agradezco su apoyo, en especial a mi abuelita Guille, sus últimas palabras fueron, "mucho éxito hija, eres mi chingona"; jamás voy a olvidar esas palabras, me dan fuerza para cada día ser una mejor persona. El tiempo a veces puede ser cruel, pero también nos enseña, seamos amigos del tiempo y disfrutemos el tiempo que pasemos juntos.

Y por eso no me queda más que decir, Gracias, y que siga la aventura... 


\section{COMITÉ TUTORIAL}

\section{Director:}

Dr. Hernando Romero Paredes Rubio. Departamento de Ingeniería de Procesos e Hidráulica. División de Ciencias Básicas e Ingeniería. Universidad Autónoma Metropolitana. 
El jurado designado por la

Comisión Académica del Posgrado en Energía y Medio Ambiente de la Unidad Iztapalapa, aprobó la tesis que presentó

Adriana Santamaria Padilla

El día 30 de mayo del año $\mathbf{2 0 1 9}$

Miembros del Jurado

M. en I. Jorge Gutiérrez Vera

Presidente

Dr. Rodolfo Vázquez Rodríguez

Secretario

M. en I. Alberto Quílez Parreño

Vocal

M. en IQ. Raimon Argemí

Puigdomènech

Vocal 
DISEÑO Y CARACTERIZACIÓN DE UN SISTEMA

MICRO-COGENERACIÓN CON

TURBINA DE GAS Y

RECUPERACIÓN DE CALOR PARA

GENERACIÓN DE VAPOR.
En la Ciudad de México, se presentaron a las 9:30 horas del día 30 del mes de mayo del año 2019 en la Unidad Iztapalapa de la Universidad Autónoma Metropolitana, los suscritos miembros del jurado:

M. EN C. JORGE GUTIERREZ VERA

M. EN C. JORGE GUTIERREZ PUIGDOMENECH

M.EN G.E. ALBERTO QUILEZ PARREÑO

DR. RODOLFO VAZQUEZ RODRIGUEZ

Bajo la presidencia del primero y con carácter de Bajo la proceder al Examen de Grado cuya denominación aparece al margen, para la de Grado cuya denominac

MAESTRA EN CIENCIAS (ENERGIA Y MEDIO AMBIENTE)

DE: ADRIANA SANTAMARIA PADILLA

y de acuerdo con el articulo 78 fracción III del Reglamento de Estudios Superiores de la Universidad Autónoma Metropolitana, los miembros del jurado resolvieron:

\section{APROBAR}

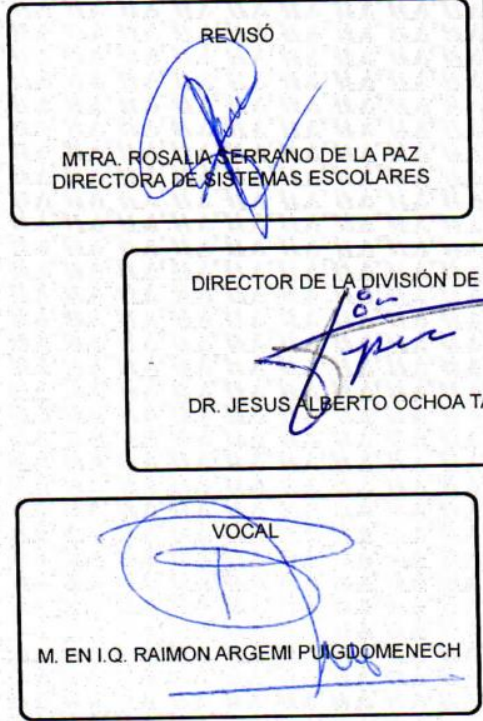

Acto continuo, el presidente del jurado comunicó a la interesada el resultado de la evaluación $y$, en caso aprobatorio, le fue tomada la protesta. 


\section{ÍNDICE GENERAL}

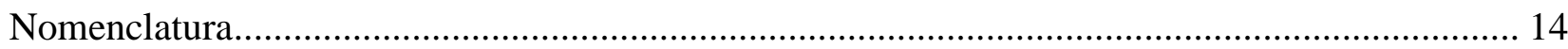

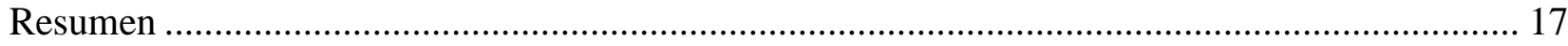

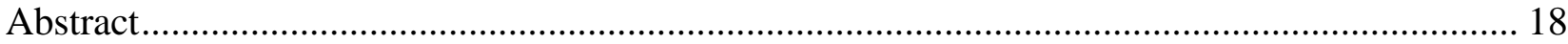

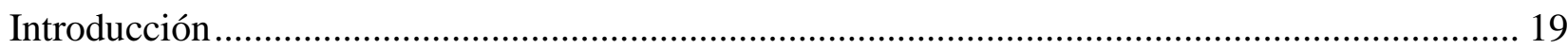

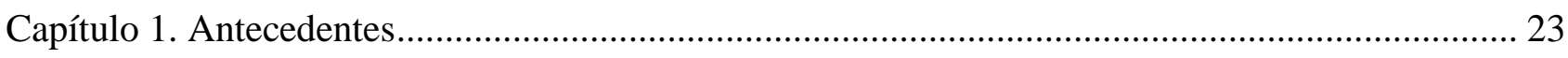

Capítulo 2. Pregunta de investigación y objetivos ........................................................................... 30

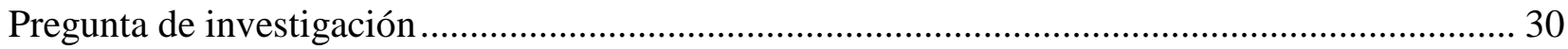

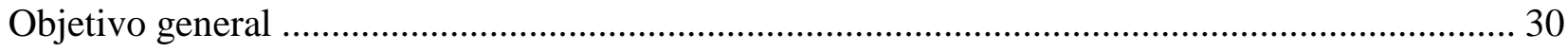

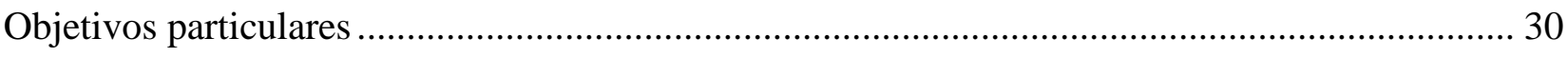

Capítulo 3. Tecnologías disponibles para Micro-cogeneración (MCHP) ............................................ 31

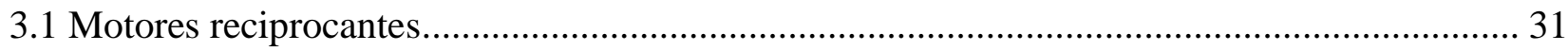

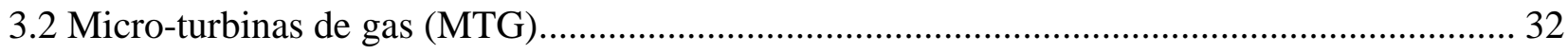

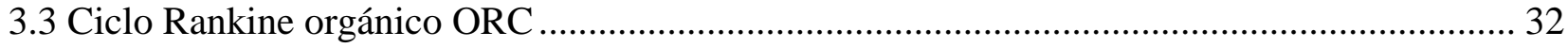

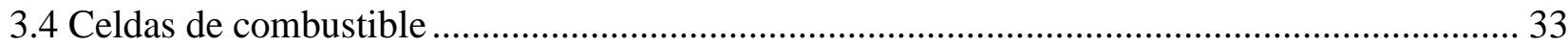

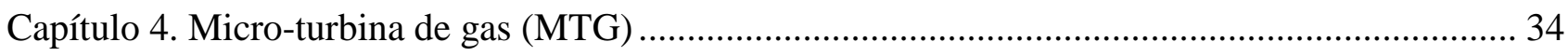

4.1 Análisis termodinámico Ciclo Brayton regenerativo ............................................................ 34

4.2 Diseño del sistema de micro-cogeneración (MCHP) con turbina de gas ................................. 36

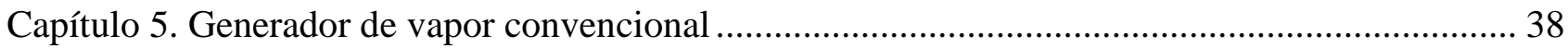

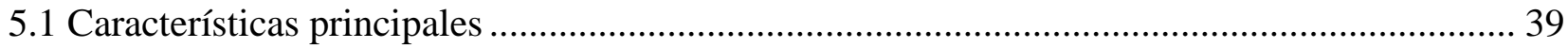

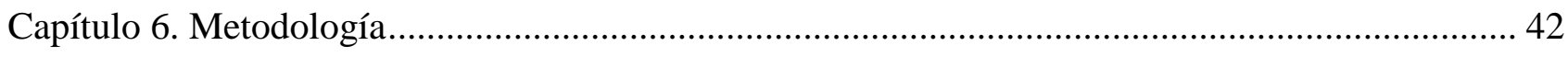

6.1 Analizar la cantidad y calidad de energía térmica disponible de las MTG de 30 y $65 \mathrm{~kW}$ con 3

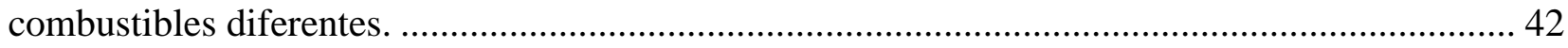

6.1.1 Gas natural y gas LP .......................................................................................... 43

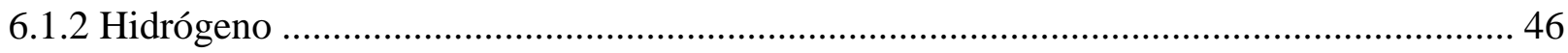

6.2 Análisis térmico de un generador de vapor convencional ..................................................... 48

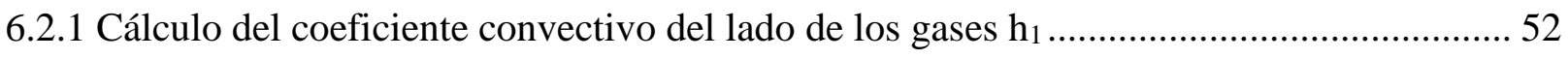

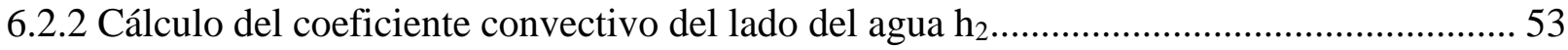

6.2.3 Cálculo del coeficiente global de transferencia de calor U ................................................ 54

6.2.4 Determinación de la caída de presión en el haz de tubos .................................................. 56

6.3 Análisis térmico de un generador de vapor que opera con los gases de combustión de una

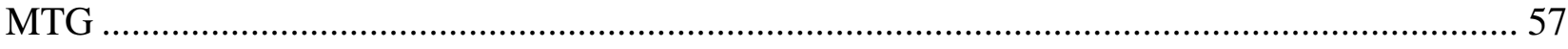

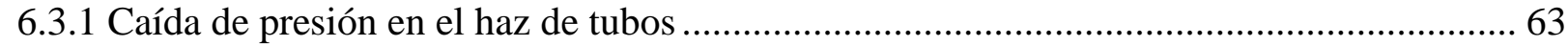




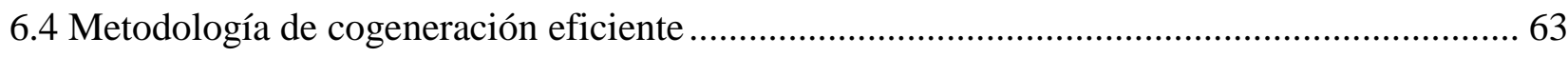

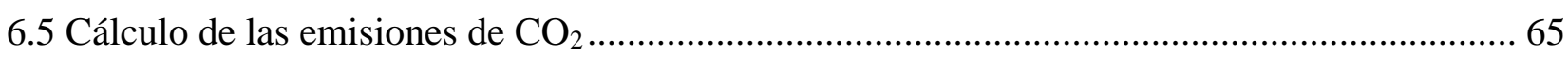

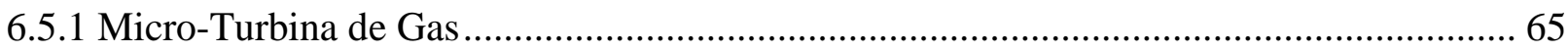

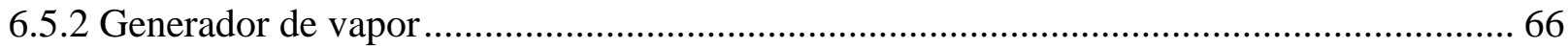

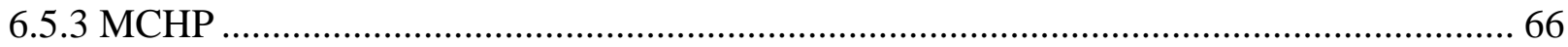

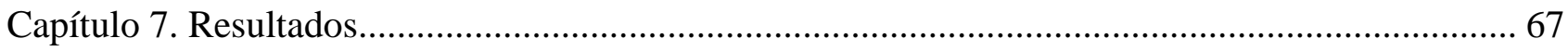

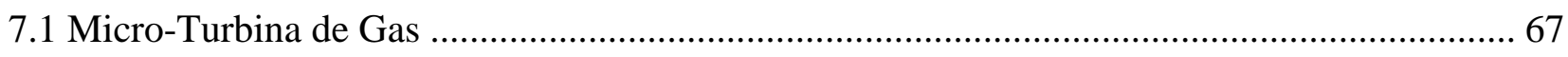

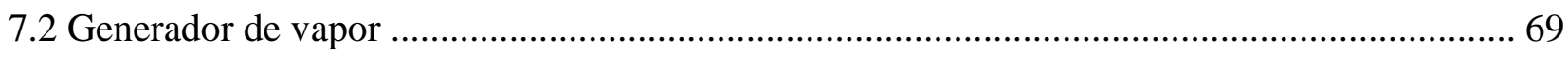

7.3 Acoplamiento de gases de combustión en un GV ............................................................... 71

7.4 Aplicación de la metodología de cogeneración eficiente a las Micro-Turbinas de Gas............ 79

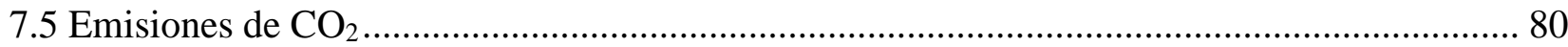

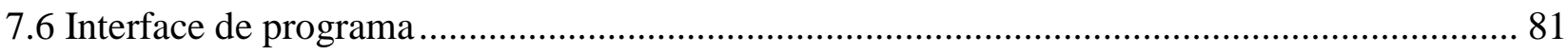

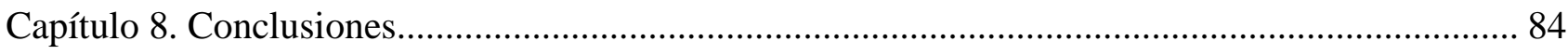

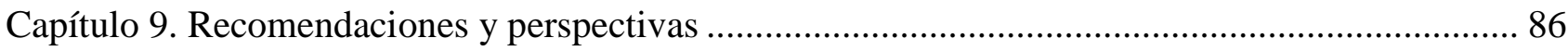

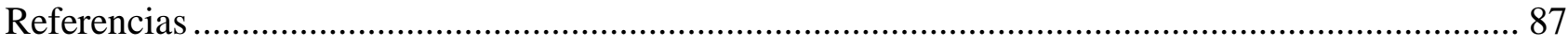

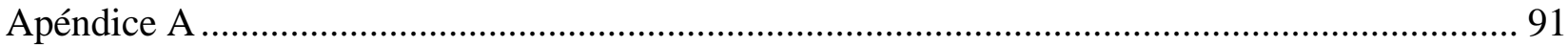

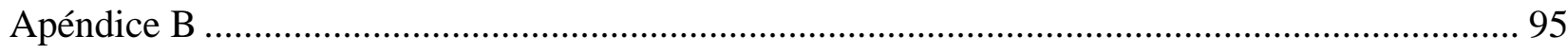

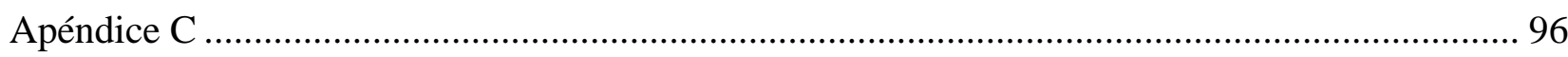

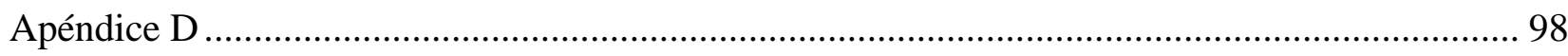

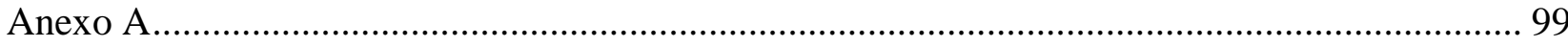

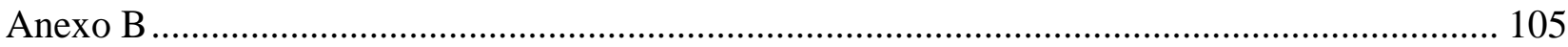

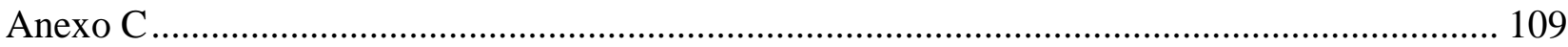

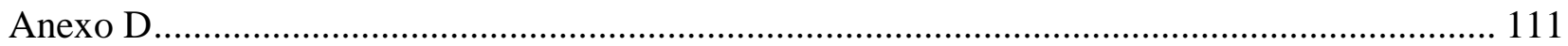




\section{ÍNDICE DE FIGURAS}

Figura 1.- Generación de $\mathrm{CO}_{2}$ equivalente en México por cada sector [7]....................................... 19

Figura 2.- Tipos de combustible y tecnología a emplear en un sistema de CHP .............................. 20

Figura 3.- Resumen de los impactos del cambio climático ............................................................... 23

Figura 4.- Generación de energía eléctrica en el año 2016 .......................................................... 24

Figura 5.- Sistema de generación de electricidad y calor convencional. .......................................... 25

Figura 6.- Sistema de generación de electricidad y calor con un sistema de cogeneración................ 25

Figura 7.- Prospectiva de la generación de energía al año 2031 ..................................................... 25

Figura 8.- Participación de la CHP en la producción total de energía nacional.................................. 27

Figura 9.- Evolución histórica de la capacidad instalada de cogeneración en México ........................ 28

Figura 10.- Diagrama esquemático de un motor de combustión interna ............................................. 31

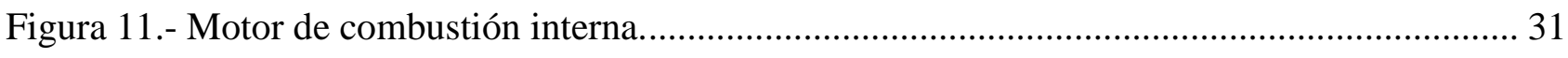

Figura 12.- Diagrama esquemático de las partes de una micro-turbina (Capstone). .......................... 32

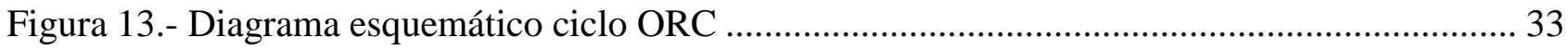

Figura 14.- Principio básico de funcionamiento de una celda de combustible................................... 33

Figura 15.- Diagrama esquemático del ciclo Brayton con regeneración ............................................. 34

Figura 16.- Infraestructura nacional de gas natural 2017 [39] .......................................................... 37

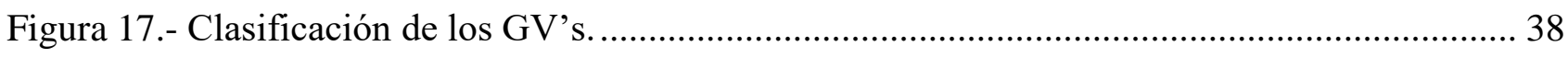

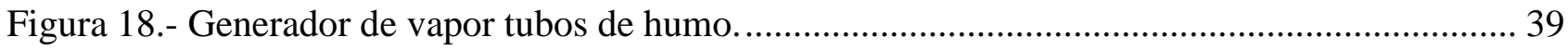

Figura 19.- Diagrama esquemático GV de 2 pasos Dry-Back ........................................................... 40

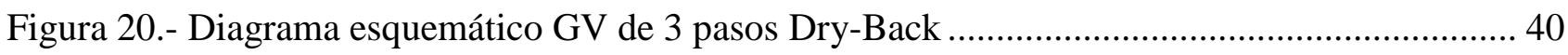

Figura 21.- Diagrama esquemático GV de 4 pasos Dry-Back ............................................................... 40

Figura 22.- Diagrama esquemático GV de 3 pasos Wet-Back........................................................... 40

Figura 23.- Diagrama esquemático GV de 4 pasos Wet-Back.............................................................. 40

Figura 24.- Temperatura de flama adiabática en función del exceso de aire ........................................ 43

Figura 25.- Diagrama Temperatura-entropía ciclo Brayton regenerativo ………………………....... 45

Figura 26.- Tipos de arreglo de tubos ......................................................................................... 49

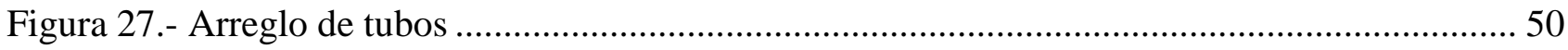

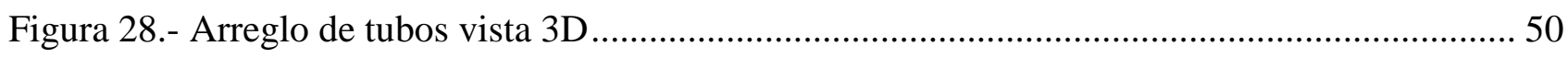

Figura 29.- Vista de los pasos de flujo ...................................................................................... 50

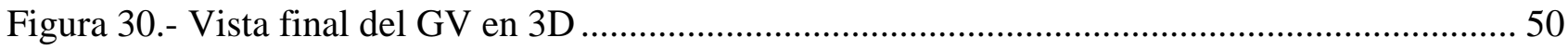

Figura 31.- Diagrama esquemático de un GV de 4 pasos Dry-Back ................................................. 51 
Figura 32.- Curva típica de ebullición del agua a 1 atm.

Figura 33.- Resistencias térmicas en coordenadas cilíndricas. .................................................... 54

Figura 34.- Ejemplo de arreglo de tubos de 4 pasos. ................................................................ 56

Figura 35.- Diagrama esquemático del acoplamiento de gases de combustión de una MTG a un GV.

Figura 36.-Diagrama de flujo para el cálculo del coeficiente convectivo de gc ............................ 62

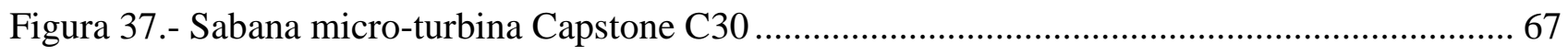

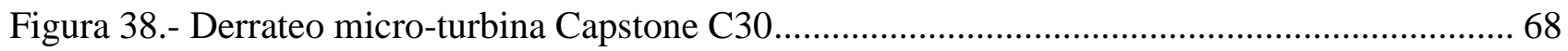

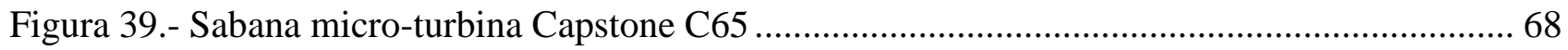

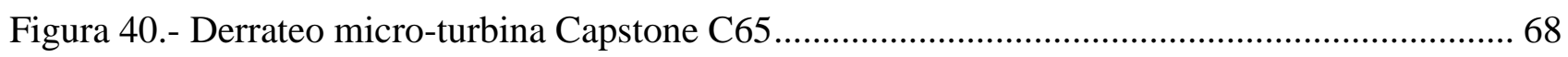

Figura 41.- Alfa en función de los GV's de estudio y \% de $\mathrm{O}_{2}$ en los gases de la MTG C30.......... 70

Figura 42.- Alfa en función de los GV's de estudio y \% de $\mathrm{O}_{2}$ en los gases de la MTG C65 .......... 70

Figura 43.- Flujo de gc del GV 80CB convencional con GN, GLP e $\mathrm{H}_{2}$ en función del exceso de aire.

Figura 44.- Coeficiente convectivo del GV 80CB convencional con GN, GLP e $\mathrm{H}_{2}$ en función del

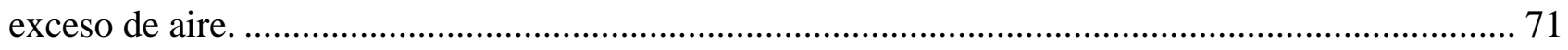

Figura 45.- Flujo de ge del GV 80CB con y sin gases provenientes de la MTG C30 con GN......... 72

Figura 46.- Flujo de gc del GV 80CB con y sin gases provenientes de la MTG C30 con GLP....... 72

Figura 47.- Flujo de ge del GV 80CB con y sin gases provenientes de la MTG C65 con GN......... 72

Figura 48.- Flujo de gc del GV 80CB con y sin gases provenientes de la MTG C65 con GLP ........ 72

Figura 49.- Flujo de ge del GV 80CB con y sin gases provenientes de la MTG C30 conH2 ........... 73

Figura 50.- Flujo de gc del GV 80CB con y sin gases provenientes de la MTG C65 conH2 ........... 73

Figura 51.- Coeficiente convectivo del GV 80CB con y sin gases provenientes de la MTG C30 con

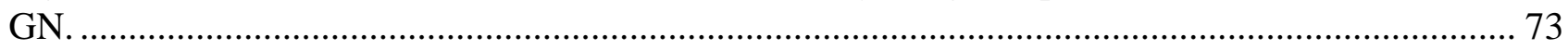

Figura 52.- Coeficiente convectivo del GV 80CB con y sin gases provenientes de la MTG C30 con GLP. 73

Figura 53.- Coeficiente convectivo del GV 80CB con y sin gases provenientes de la MTG C65 con

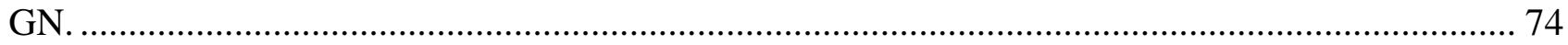

Figura 54.- Coeficiente convectivo del GV 80CB con y sin gases provenientes de la MTG C65 con

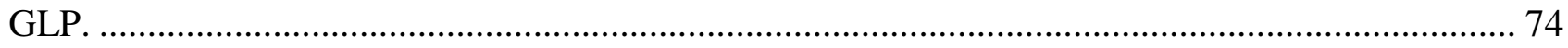

Figura 55.- Coeficiente convectivo del GV 80CB con y sin gases provenientes de la MTG C30 con

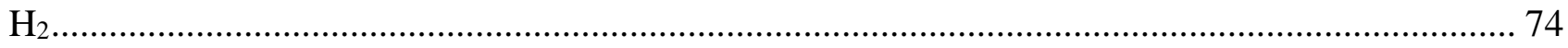

Figura 56.- Coeficiente convectivo del GV 80CB con y sin gases provenientes de la MTG C65 con

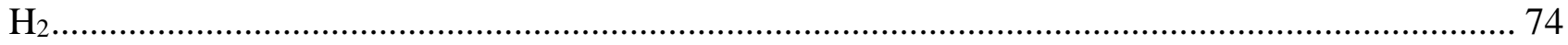


Figura 57.- Coeficientes convectivos $\mathrm{h}_{1} \mathrm{y} \mathrm{h}_{2}$ para cada $\mathrm{GV}$ de estudio con los gases provenientes de la MTG C30 con GN.

Figura 58.- Coeficientes convectivos $\mathrm{h}_{1} \mathrm{y} \mathrm{h}_{2}$ para cada $\mathrm{GV}$ de estudio con los gases provenientes de la MTG C650 con GN.

Figura 59.- Coeficiente global de transferencia de calor del GV 80CB con y sin gases provenientes de la MTG C30 con GN. 75

Figura 60.- Coeficiente global de transferencia de calor del GV 80CB con y sin gases provenientes de la MTG C30 con GLP 75

Figura 61.- Coeficiente global de transferencia de calor del GV 80CB con y sin gases provenientes de la MTG C65 con GN 75

Figura 62.- Coeficiente global de transferencia de calor del GV 80CB con y sin gases provenientes de la MTG C65 con GLP 75

Figura 63.- Coeficiente global de transferencia de calor del GV 80CB con y sin gases provenientes de la MTG C3O con $\mathrm{H}_{2}$... 76

Figura 64.- Coeficiente global de transferencia de calor del GV 80CB con y sin gases provenientes de la MTG C65 con $\mathrm{H}_{2}$. 76

Figura 65.- Coeficiente convectivo para la gama de GV's usando los gases provenientes de la MTG C30 con GN......

Figura 66.- Coeficiente convectivo para la gama de GV's usando los gases provenientes de la MTG C65 con GN..

Figura 67.- Coeficiente global de transferencia de calor para la gama de GV's usando los gases provenientes de la MTG C30 con GN.

Figura 68.- Coeficiente global de transferencia de calor para la gama de GV's usando los gases provenientes de la MTG C65 con GN.

Figura 69.- Caída de presión en el GV 80CB empleando los gases efluentes de la MTG C30........ 77

Figura 70.- Caída de presión en el GV 80CB empleando los gases efluentes de la MTG C65......... 77

Figura 71.- Interface del programa realizado en Excel ..................................................... 82

Figura 72.- Interface de cálculo para la metodología de cogeneración eficiente........................... 83

Figura 73.- Diagrama de flujo para el cálculo de la viscosidad de los gc ................................... 91

Figura 74.- Diagrama de flujo para el cálculo de la conductividad térmica de los gc .................... 91

Figura 75.- Integral de colisión $\Omega v$ para la viscosidad y de la función de temperatura y viscosidad.94

Figura 76.- Nusselt con GN en función del exceso de aire usando la MTG C30 .......................... 96

Figura 77.- Conductividad térmica con GN en función del exceso de aire usando la MTG C30...... 96

Figura 78.- Nusselt con GN en función del exceso de aire usando la MTG C65 .......................... 97

Figura 79.- Conductividad térmica con GN en función del exceso de aire usando la MTG C65...... 97 


\section{ÍNDICE DE TABLAS}

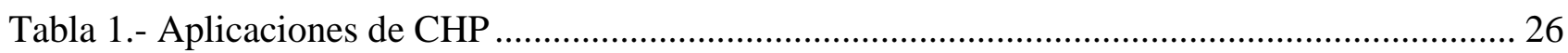

Tabla 2.- Ventajas y desventajas de los GV's tubo de humo............................................................ 39

Tabla 3.- Especificaciones de las micro-turbinas de estudio. .............................................................. 42

Tabla 4.- Composición de cada uno de los combustibles ................................................................... 42

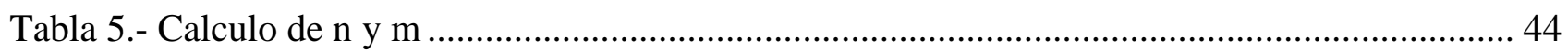

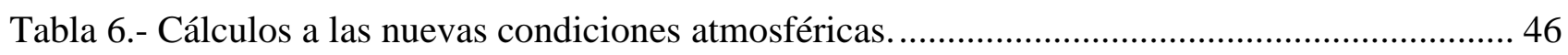

Tabla 7.- Cálculos a las nuevas condiciones atmosféricas usando $\mathrm{H}_{2}$.................................................. 48

Tabla 8.- Especificaciones de GV's Cleaver Brooks de 4 paso Dry-Back de 15 a 100 BHP ............ 50

Tabla 9.- Correlaciones del factor de fricción [49]......................................................................... 57

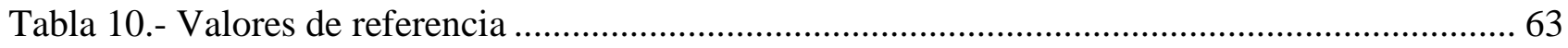

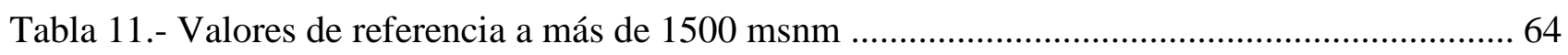

Tabla 12.- Factor de perdida de acuerdo al nivel de tensión.................................................................... 64

Tabla 13.- Resultados con los 3 combustibles en condiciones ISO ................................................... 69

Tabla 14.- Resultados con los 3 combustibles en condiciones atmosféricas de la CDMX................. 69

Tabla 15.- Evaluación de $\alpha$ para cada MTG y GV de estudio ........................................................... 70

Tabla 16.- Potencia del soplador mínima requerida en el GV. ........................................................ 77

Tabla 17.- Eficiencias globales del sistema convencional vs MCHP ................................................. 78

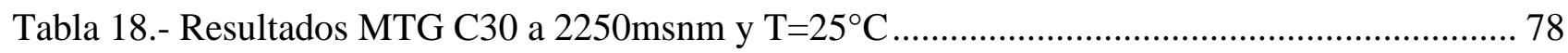

Tabla 19.- Resultados del GV 20CB usando los gases de combustión de la MTG C30..................... 78

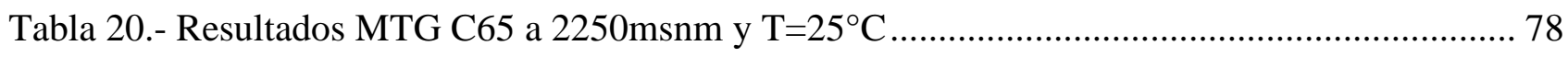

Tabla 21.- Resultados del GV 20CB usando los gases de combustión de la MTG C65...................... 78

Tabla 22.- Resultados de las eficiencias del sistema convencional vs MCHP usando el GV 20 CB. 79

Tabla 23.- Resultados obtenidos de la metodología de cogeneración eficiente para la MTG C30 con 3

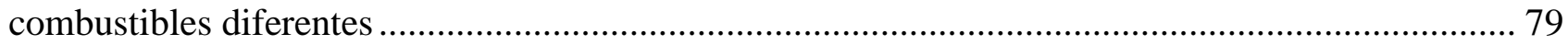

Tabla 24.- Resultados obtenidos de la metodología de cogeneración eficiente para la MTG C65 con 3

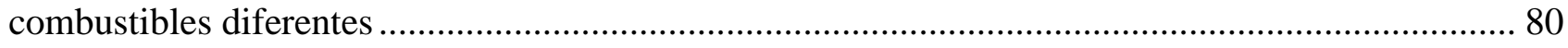

Tabla 25.- Toneladas de $\mathrm{CO}_{2}$ por equipo y combustible emitidas (MTG C30) ................................. 81

Tabla 26.- Toneladas de $\mathrm{CO}_{2}$ por equipo y combustible emitidas (MTG C65) ................................. 81

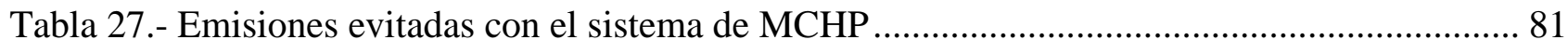

Tabla 28.- Propiedades de Lennard-Jones determinados a partir de datos de viscosidad................... 92

Tabla 29.- Valores de la integral de colisión $\Omega$ v para la viscosidad y de la función de temperatura y viscosidad. 
Tabla 30.- Capacidad calorífica de diferentes compuestos....................................................... 95

Tabla 31.- Variación del Nusselt y la Conductividad térmica con respecto al exceso de aire con MTG

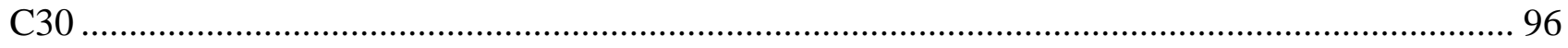

Tabla 32.- Variación del Nusselt y la Conductividad térmica con respecto al exceso de aire con MTG

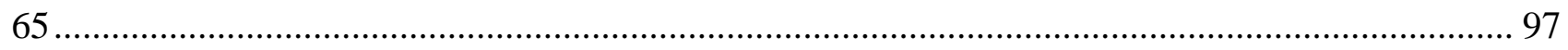

Tabla 33.- Valores de referencia de Gorenflo (1993) con $\alpha_{0}$ en $\mathrm{W} / \mathrm{m}^{2} \mathrm{~K}$ a $\mathrm{P}_{\mathrm{r} 0}=0.1, \mathrm{q}_{0}=20,000 \mathrm{Wm} 2 \mathrm{y}$

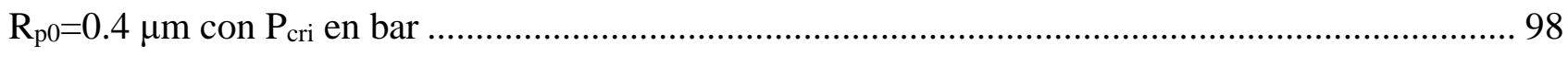

Tabla 34.- Antecedentes de micro cogeneración en el mundo................................................. 103 


\section{Nomenclatura}

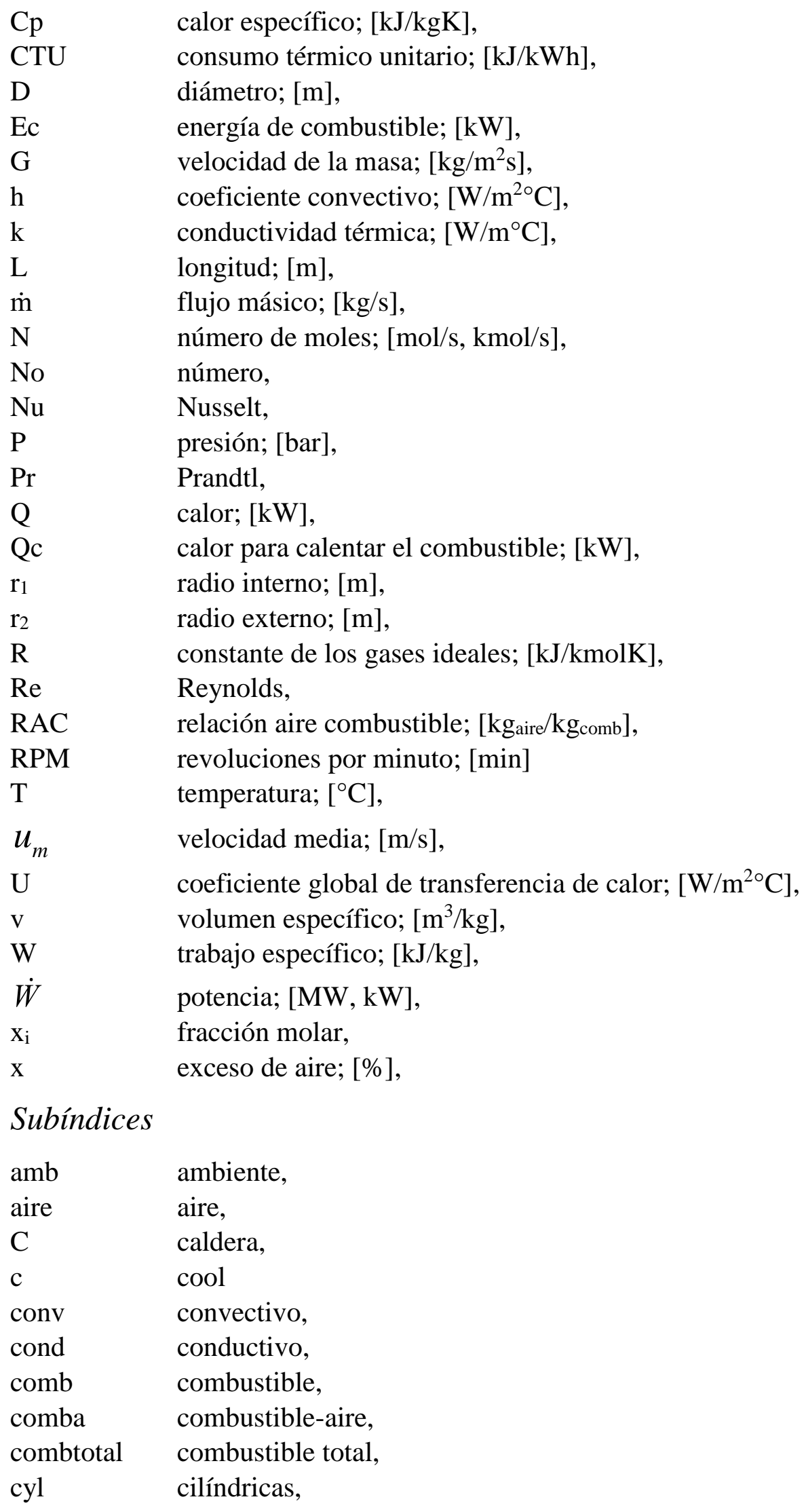




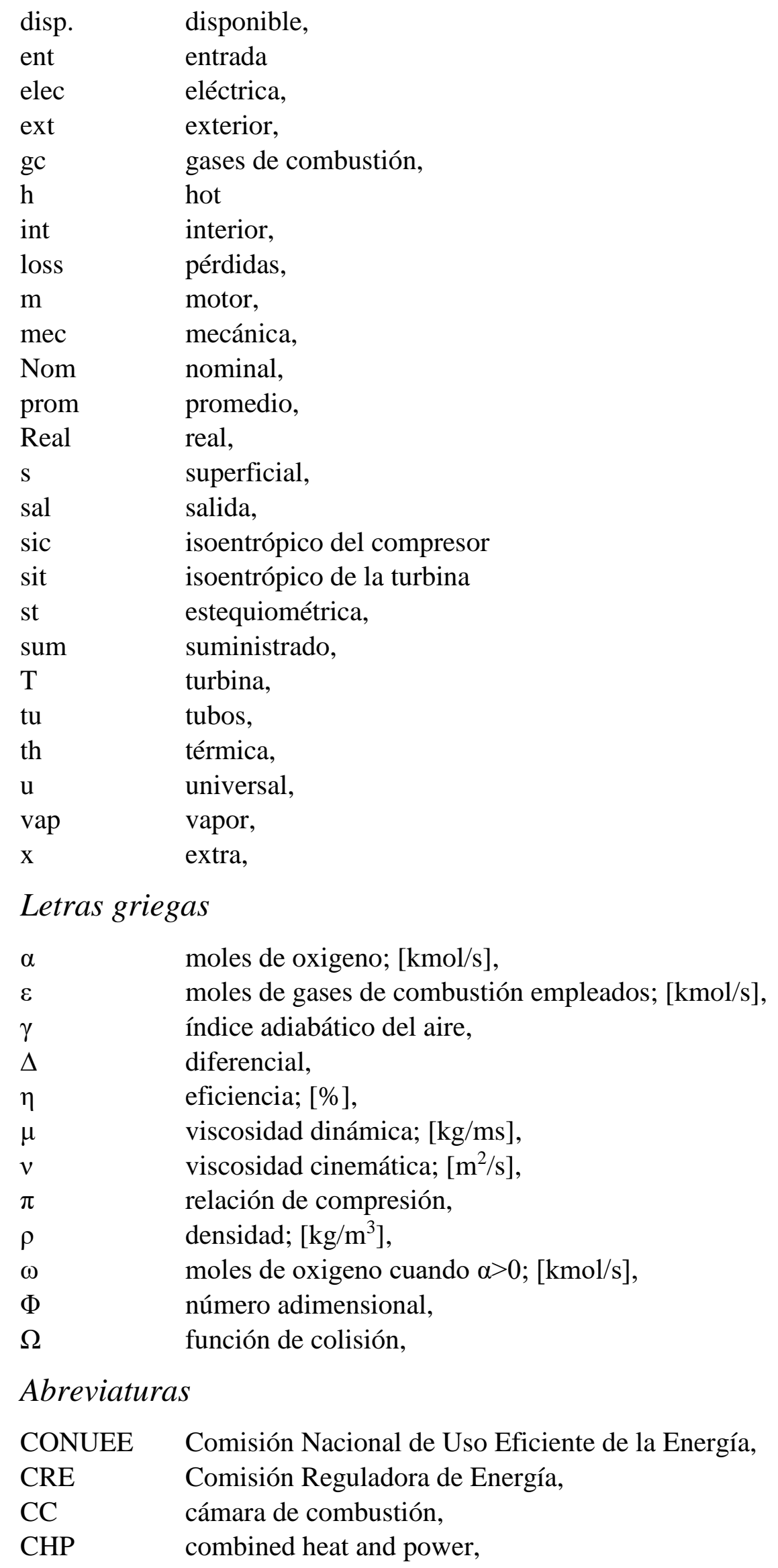

\section{Abreviaturas}

CONUEE Comisión Nacional de Uso Eficiente de la Energía,

CRE Comisión Reguladora de Energía,

CC cámara de combustión,

CHP combined heat and power, 
CCGT combined cycle gas turbine,

DHC District Heating and Cooling,

GIZ Deutsche Gesellschaft für Internationale Zusammenarbeit,

GE generador eléctrico,

GEI gases de efecto invernadero,

GN gas natural,

GLP gas licuado de petróleo,

GV generador de vapor,

$\mathrm{H}_{2} \quad$ hidrógeno,

IPCC Intergovernmental Panel on Climate Change,

LIE Ley de la industria eléctrica,

LGCC Ley General de Cambio Climático,

LTE Ley de Transición Energética,

MCHP micro combined heat and power,

MTG micro turbina de gas,

MM masa molecular,

OCDE Organización para la Cooperación y el Desarrollo Económicos, PCI poder calorífico inferior,

RENE Registro Nacional de Emisiones,

SENER Secretaria de Energía

SEMARNAT Secretaría de Medio Ambiente y Recursos Naturales,

TET temperatura de entrada en la turbina, 


\section{Resumen}

En este proyecto se propone abordar la problemática de las emisiones de GEI emitidas por los sectores industrial y generación de energía eléctrica. En la actualidad, la generación de energía en nuestro país se basa fundamentalmente en el consumo de combustibles fósiles. Es por ello que se busca el desarrollo de una política energética integral que haga especial énfasis en los programas de eficiencia energética a nivel nacional y regional y, a la vez, promueva la diversificación y la utilización de energías alternas y renovables [1].

La cogeneración, o producción combinada de calor y electricidad (CHP), es "el proceso de producir electricidad y energía térmica utilizable (calor y / o refrigeración) con alta eficiencia y cerca del usuario"[2]. Incorpora cuatro elementos: 1) la producción simultánea de electricidad y calor; 2) un criterio de rendimiento de alta eficiencia; 3) la reducción de emisiones de GEI; y 4) un criterio de localización relativo a la proximidad de la unidad de conversión de energía a un usuario [3]. Este tipo de sistemas pueden llegar a tener eficiencias superiores al 80\% [4] lo cual hace que sean una alternativa tecnológica atractiva. La micro-cogeneración es llamada así, por tratarse de pequeñas unidades de generación eléctrica $(<200 \mathrm{~kW})$ en las que, frecuentemente, se les acopla una caldera de recuperación de calor. Es posible reducir hasta 1000 toneladas de $\mathrm{CO}_{2}$ por $\mathrm{GWh}$ de energía generada, dependiendo de las fuentes de calor y electricidad desplazadas y el tiempo de uso [3].

El empleo de los gases de escape de una micro-turbina de gas (MTG) pueden ser usados para la alimentación de aire primario de un generador de vapor convencional $(\mathrm{GV})$, lo cual es factible debido a que estos vienen con un elevado exceso de aire y alta temperatura que puede sustituir el aire normal de alimentación para la combustión y en principio no perturban el proceso de combustión. Bajo esta premisa se reduce el consumo de combustible. Para ello se requieren hacer estudios de evaluación teórica que permitan conocer si al hacer estos cambios en el GV, el proceso de la combustión se ve afectado, el quemador soporta las nuevas condiciones de operación y determinar los efectos que se tendrán en los coeficientes de transferencia de calor en el haz de tubos y en la eficiencia del GV.

Por lo tanto, el objetivo general de esta tesis es: Diseñar y evaluar un sistema de microcogeneración cuando los gases efluentes de la MTG se inyectan a un GV pirotubular pre-existente.

En este trabajo se evaluaron dos MTG la C30 y C65, ambas de la marca Capstone y la gama de GV's 15-100 CB de Cleaver Brooks. 


\section{Abstract}

In this project, it is proposed to address the problem of GHG emissions emitted by the industrial and electric power generation sectors. At present, the power generation in our country is based fundamentally on fossil fuels. For this reason, the development of an integral energy policy that makes special emphasis on energy efficiency programs at the national and regional level is sought and, at the same time, promotes the diversification and the use of alternative and renewable energies [1]. Cogeneration, or combined production of heat and power (CHP), is "the process of producing electricity and usable thermal energy (heat and/or cooling) with high efficiency and close to the user" [2]. It incorporates four elements: 1) the simultaneous production of electricity and heat; 2) a high efficiency performance criterion; 3) the reduction of GHG emissions; and 4) a location criterion relative to the proximity of the energy conversion unit to a user [3]. This type of systems can reach efficiencies higher than $80 \%$ [4], which makes them an attractive technological alternative. The microcogeneration is called like this, for being small units of electrical generation $(<200 \mathrm{~kW})$ in which, frequently, a heat recovery steam generator (HRSG) is coupled to it. It is possible to reduce up to 1000 tons of $\mathrm{CO} 2$ per GWh of generated energy, depending on the sources of heat and electricity displaced and the time of use [3]. The use of the exhaust gases of a micro gas turbine (MGT) can be used for the primary air supply of a conventional steam boiler, which is feasible because the gases come with a high excess air and high temperature that can replace the normal feed air for combustion. In principle, it does not disturb the combustion process. Under this premise, fuel consumption is reduced. However, theoretical evaluation studies are required to know and evaluate if combustion is affected when making these changes in the conventional steam boiler. Additionally, it will be needed to study the effects on the coefficients of heat transfer in the tube bundles and their efficiency. Therefore, the

general objective of this thesis is: Design and evaluate a micro-cogeneration system when the MTG effluent gases are injected into a pre-existing pirotubular steam boiler. In this work two MTGs were evaluated the $\mathrm{C} 30$ and C65, both of the Capstone brand and the range of steam boilers 15-100 CB by Cleaver Brooks. 


\section{Introducción}

El uso desmesurado de los combustibles fósiles y el uso ineficiente de la energía ha provocado que las emisiones de gases de efecto invernadero (GEI) se incrementen, lo que genera un aumento en la temperatura media del planeta. Entre 1880 y 2012, la temperatura anual global tuvo un incremento de $0.85^{\circ} \mathrm{C}$ con respecto al promedio del periodo de 1961-1990 [5]. Al fenómeno de incremento global de temperatura se le conoce como calentamiento global y este puede ser de origen natural y antropogénico, como consecuencia de éste se tiene la existencia del cambio climático.

El cambio climático es considerado uno de los problemas ambientales más importantes de nuestro tiempo y puede definirse como todo cambio significativo en el sistema climático del planeta que permanece por décadas o más tiempo. Además, es una amenaza para la seguridad alimentaria mundial, el desarrollo sostenible y la erradicación de la pobreza. Los GEI producidos por la actividad humana son el motor más significativo del cambio climático [6].

Las emisiones de GEI están estrechamente vinculadas a la actividad económica en los diferentes sectores industriales, comerciales y de servicios del país, que se refleja en la contribución de cada sector de la sociedad a las emisiones nacionales. En la Figura 1 se muestra que el sector transporte aporta el $24.9 \%$ seguido de la industria con un $23.2 \%$, y la generación de electricidad con un $18 \%$, como los principales emisores de GEI [7].

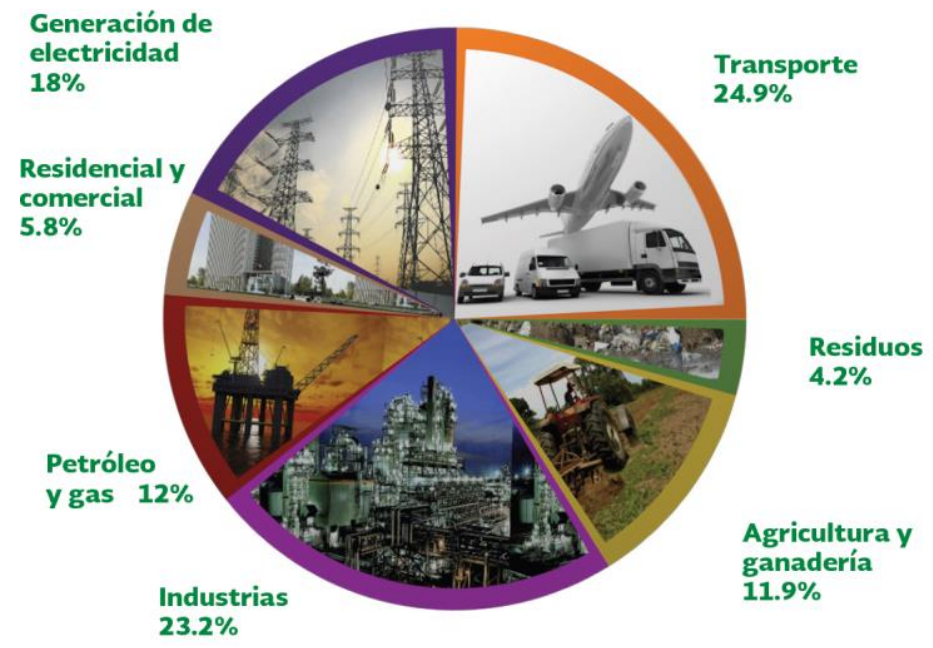

Figura 1.- Generación de $\mathrm{CO}_{2}$ equivalente en México por cada sector [7].

En la actualidad, la generación de energía en nuestro país se basa fundamentalmente en el consumo de combustibles fósiles. Es por ello que se busca el desarrollo de una política energética integral que haga especial énfasis en los programas de eficiencia energética a nivel nacional y, a la vez, promueva la diversificación y la utilización de energías alternas y renovables [1].

De acuerdo con la Ley General de Cambio Climático (LGCC), México tiene como meta reducir la emisión de los GEI en un 30\% para el 2020 y en un $50 \%$ para el 2050 respecto a las emitidas en el 2000 [8]. Para cumplir con dicha meta es necesario involucrar a los distintos sectores productivos del país, con el fin de generar acciones, proyectos, políticas y estrategias conjuntas y corresponsables que permitan atender la problemática que demanda el cambio climático [7].

La dependencia mundial de los combustibles fósiles es probable que se sitúe en torno al $90 \%$ en 2020, a menos que se adopten medidas importantes para introducir las energías renovables en el 
sistema energético [3], para fortalecer las acciones en eficiencia energética de los sistemas, procesos y equipos, entre las que destacan por su gran impacto los sistemas de cogeneración, para los sectores industrial, comercial y de servicios. El principio de cogeneración se conoce desde hace tiempo. En la primera década del siglo XX, varias unidades de cogeneración ya estaban suministrando calor y electricidad a casas y empresas. La cogeneración, o producción combinada de calor y electricidad (CHP), es "el proceso de producir electricidad y energía térmica utilizable (calor y/o refrigeración) con alta eficiencia y cerca del punto de uso" [2]. Incorpora así cuatro elementos: 1) la producción simultánea de electricidad y calor; 2) un criterio de rendimiento de alta eficiencia; 3) la reducción de emisiones de GEI; y 4) un criterio de localización relativo a la proximidad de la unidad de conversión de energía a un usuario o un cliente [3]. En la mayoría de las veces se establecen como sistemas distribuidos; aquí, usuario y generador son uno mismo. La cogeneración y particularmente a pequeña escala, llamada micro-cogeneración, está orientada al usuario para usar con mayor eficiencia los combustibles fósiles convencionales y permita con esa tecnología contribuir a la mitigación del calentamiento global, el desarrollo de un sector energético más sólido, competitivo y sustentable.

Uno de los grandes problemas de la industria nacional es la ineficiencia en el uso de la energía. Sin embargo, su generación para un uso óptimo de la energía no siempre se vincula con su uso. De ahí la importancia de los sistemas de cogeneración. En la generación distribuida se utilizan distintas tecnologías que se basan en los primotores: turbinas de gas, motores alternativos, turbinas de vapor. También pueden emplearse pilas de combustible, sistemas solares y maquinas eólicas (Figura 2).

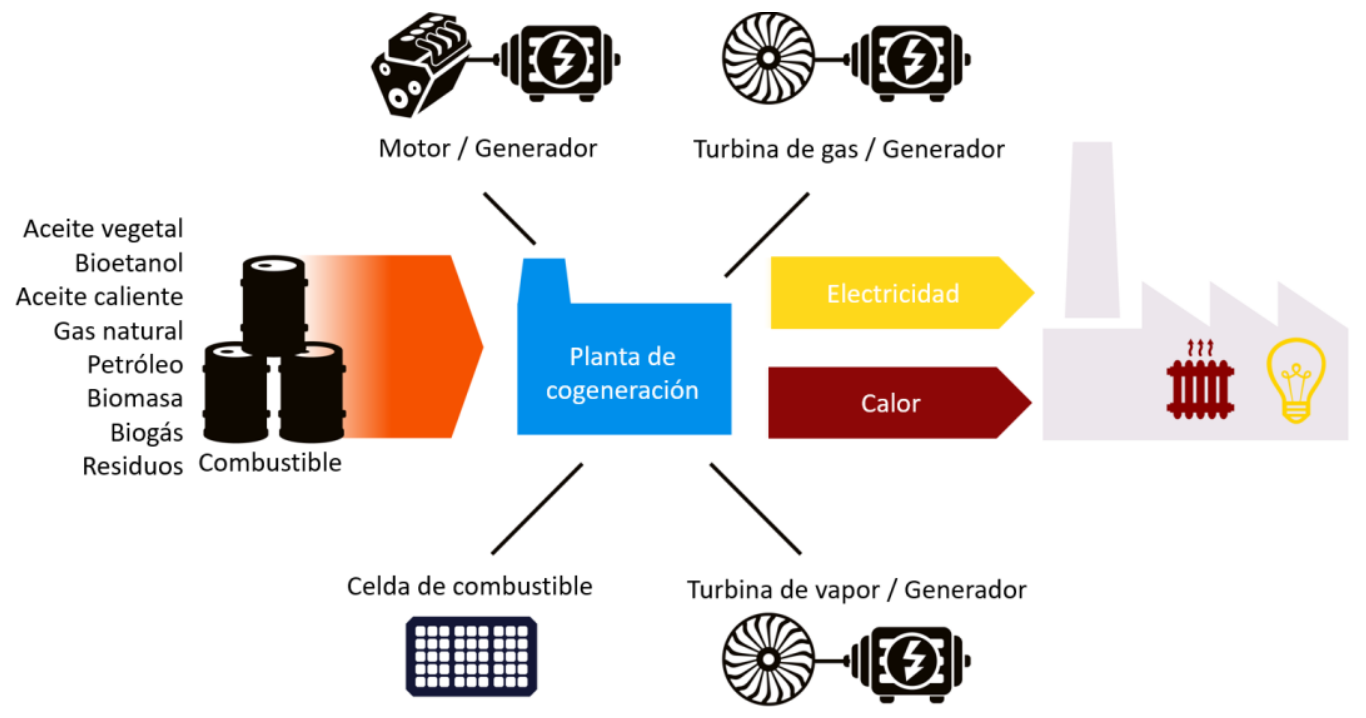

Figura 2.- Tipos de combustible y tecnología a emplear en un sistema de CHP

Existe un interés particular en el empleo potencial de tecnologías como micro-turbinas de gas (MTG) y motores alternativos. El uso de turbinas de gas para la generación de energía ha aumentado en los últimos años y es probable que continúe su incremento. La proporción de la generación de energía mediante CHP también está creciendo principalmente debido a las mejoras en las eficiencias y beneficios ambientales[9] como son:

- Disminución de los consumos de energía primaria.

- Disminución de las importaciones de combustible (ahorros en la balanza de pagos del país)

- Reducción de emisiones de GEI.

- Disminución de pérdidas en el sistema eléctrico e inversiones en transporte y distribución. 
- $\quad$ Aumento de la garantía de potencia y calidad del servicio eléctrico.

- Aumento de la competitividad industrial y de la competencia en el sistema eléctrico.

- Promoción de pequeñas y medianas empresas de construcción y operación de plantas de cogeneración.

- Adaptabilidad en zonas aisladas o ultraperiféricas.

- Motivación por la investigación y desarrollo de sistemas energéticos eficientes.

- Es posible reducir hasta 1000 toneladas de $\mathrm{CO}_{2}$ por $\mathrm{GWh}$ de potencia, dependiendo de las fuentes de calor y electricidad desplazadas [10].

En esta época en la que los ecosistemas se han visto afectados por las emisiones contaminantes de todo tipo de sustancias que los dañan y en el que la combustión de los combustibles fósiles participa en un poco más del $70 \%$ de esas emisiones y contribuyen sustancialmente en el calentamiento global, es de suma importancia que su empleo se haga con la mayor eficiencia posible. Es por ello que los sistemas de cogeneración y de micro-cogeneración que pueden llegar a tener eficiencias superiores al $80 \%$ [4] son una alternativa atractiva para hacer regiones sustentables.

Existen instalaciones comerciales, industriales de pequeña escala y en el sector residencial en donde los sistemas de micro-cogeneración (MCHP) pueden ser una solución viable para un mejor aprovechamiento de los recursos naturales. El potencial en estos sectores no ha sido valorado en toda su extensión. Existe una cierta incertidumbre en cuanto a la capacidad que puede existir en México y por ello se deben de emprender diferentes acciones que permitan estimar el potencial de esta tecnología [1]. Entre estas acciones destacan la necesidad de realizar "un mapa de calor" en el país. Éste es la descripción regional del uso de combustibles fósiles que se emplean para calentamiento; naturalmente no debe de incluir el empleado en la generación eléctrica a gran escala. Otra acción es el desarrollo de proyectos demostrativos en los que compruebe la factibilidad técnica y económica de estos sistemas de MCHP.

Para el estudio de la factibilidad técnica en una instalación determinada es fundamental el tiempo de uso de cada una de las formas de energía: eléctrica y térmica. Entre mayor tiempo anual de uso se tenga la probabilidad que pueda ser viable se incrementa para este tipo de instalaciones (entre 5,000 y 8,000 hrs/año).

La tecnología disponible en el mercado está muy acotada. En ese sentido la turbina de gas más pequeña es de $30 \mathrm{~kW}$. Anteriormente había 6 marcas de MTG disponibles: Allied, Elliot/Bowman, Capstone, Power Works, ABB/Turbec y Honeywell [11], hoy en día solo existe la Capstone y Ansaldo Energía cuya capacidad inferior es de $30 \mathrm{~kW}$ y $100 \mathrm{~kW}$. En materia de motores alternativos no hay mucha diferencia. El motor alternativo más pequeño que puede ser empleado para la microcogeneración es de $20 \mathrm{~kW}$ y las marcas disponibles en el mercado son: Caterpillar, Waukesha, Kholer Co., Jenbacher, Cummins, Detroit Diesel, Cooper Energy Services [12][13].

Una de las maneras de desarrollar los sistemas de MCHP con mayor celeridad es empleando los gases efluentes de una MTG para diferentes aplicaciones, algunas de ellas son: el calentamiento de agua, producción de vapor, refrigeración por absorción, calefacción, entre otras [14]. Las industrias que tienen el mayor potencial de mercado estimado para el uso de MTG en MCHP son: alimentos, bebidas, pulpa y papel, textiles, hospitales, hoteles y edificios institucionales.

Los gases de combustión de una MTG salen a altas temperaturas, alrededor de los 270 a 320 ${ }^{\circ} \mathrm{C}$ [15], dependiendo de la potencia de esta, los cuales en sistemas convencionales son mandados 
directamente a la atmósfera. Razón por la cual es importante realizar un análisis de factibilidad de la implementación de un sistema continuo de aprovechamiento de los gases de combustión de la MTG en un generador de vapor (GV) convencional que evitará la inversión de un recuperador de calor.

Este proyecto está orientado al análisis de factibilidad de emplear una MTG y acoplar sus gases de escape a un GV convencional debido a que esos gases vienen con un elevado exceso de aire que en principio no perturban el proceso de combustión convencional del GV. Bajo esta premisa se reduce el consumo de combustible. Se debe por lo tanto conocer la magnitud de la perturbación en los coeficientes de transferencia de calor en el haz de tubos al sustituir el aire de combustión y reducir el consumo.

Este trabajo de tesis está en concordancia con los objetivos del posgrado de Energía y Medio Ambiente, pues está orientado a los dos aspectos que dan nombre al posgrado: energía y medio ambiente. Energía porque uno de los objetivos de este trabajo es desarrollar un modelo teórico de un sistema de micro-cogeneración que emplea los gases de combustión para sustituir o, en su caso, complementar los quemadores convencionales de un calentador de agua o generador de vapor para hacer el sistema más eficiente. Medio ambiente porque se orienta a reducir las emisiones de gases efecto invernadero (GEI) por kWh generado (eléctrico y térmico) contribuyendo a la mitigación del cambio climático. En el ámbito energético se puede aprovechar diversas materias primas; combustibles fósiles y renovables como el biogás, gas de síntesis etc, y generar en conjunto energía eléctrica y térmica, haciendo que este sea un sistema energético eficiente y sustentable. 


\section{Capítulo 1. Antecedentes}

El cambio climático ha ocasionado grandes impactos en los ecosistemas, la sociedad y los sectores productivos. Uno de los principales indicios de la existencia del cambio climático es el incremento de la temperatura media anual global. En la Figura 3 se observan las principales afectaciones a nivel mundial identificados por el grupo Intergubernamental de expertos sobre el cambio climático (IPCC, por sus siglas en inglés) en los diferentes sistemas: físicos, biológicos y gestionados; muestra de que el cambio climático tiene un gran impacto [16].

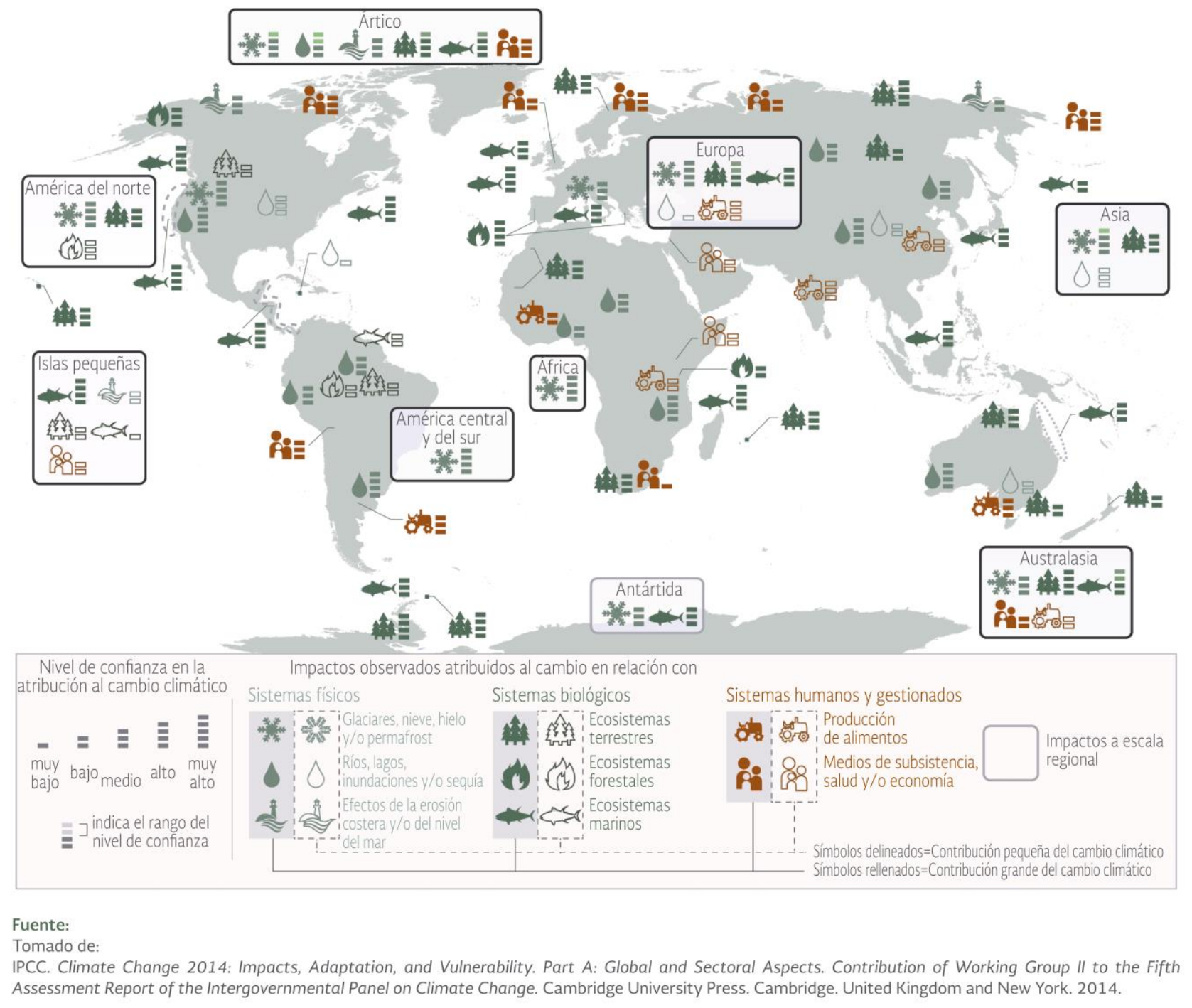

Figura 3.- Resumen de los impactos del cambio climático

En México casi el $80 \%$ de la generación de energía proviene de los combustibles fósiles (Figura 4) [17], que contribuyen de manera importante en los importantes impactos mostrados en la Figura 3. El uso de estos combustibles son por consecuencia un factor sustancial para el calentamiento global. De esta premisa se deriva la importancia de que su uso se haga de la manera más eficiente posible. 


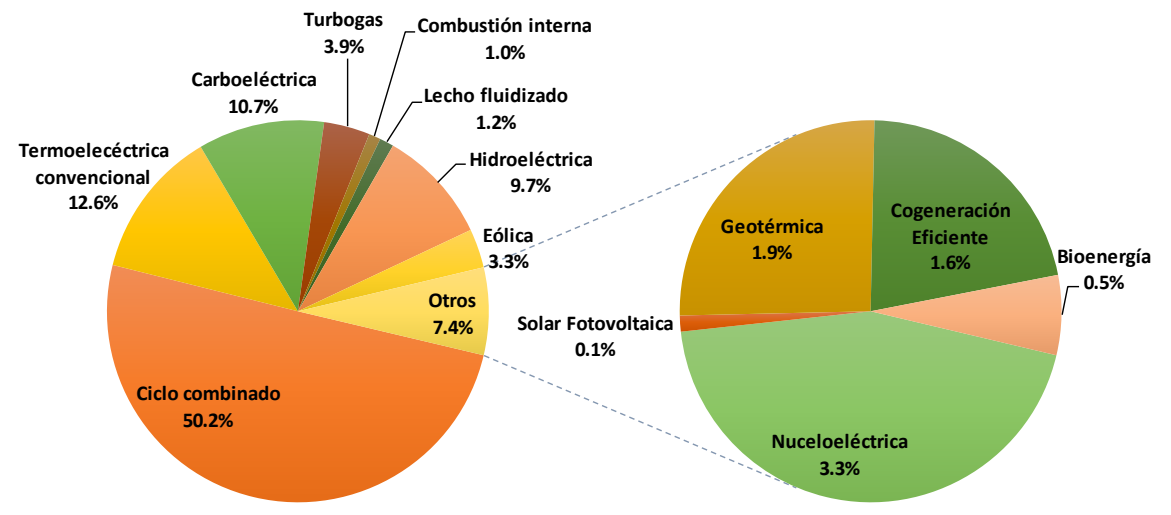

Figura 4.- Generación de energía eléctrica en el año 2016

Para llevar a cabo una transición energética exitosa se necesita tener una estrategia para promover el uso de tecnologías y combustibles más limpios en el mediano y largo plazo. Las principales componentes para hacer posible este cambio son: la eficiencia energética y el uso de energías renovables [17]. De acuerdo al reporte de avance de energías limpias para el primer semestre de 2018, estas representan el $24.12 \%$ de la generación total, menos de un punto porcentual para cumplir con la meta del 25\% de generación de energía limpia establecida por México en la Ley de transición Energética. Donde la cogeneración eficiente aporta un 2.79\% [18].

El consumo de energía térmica en el país representa alrededor de 77\% del consumo energético total. El aprovechamiento del calor residual se emplea parcialmente en algunas industrias, pero no es una práctica común.

Aunque los sistemas de cogeneración son en general muy eficientes, éstos no están muy difundidos en México, pese a que en el pasado ha habido programas para su difusión e implementación. La cogeneración eficiente de acuerdo con la Ley de transición energética, artículo 3 , fracción VI, publicada en el diario oficial de la federación el 24 de diciembre de 2015 [19]:

I. Producción de energía eléctrica conjuntamente con vapor u otro tipo de energía térmica secundaria, o ambas.

II. Producción directa o indirecta de energía eléctrica a partir de energía térmica no aprovechada en los procesos de que se trate.

III. Producción directa o indirecta de energía eléctrica utilizando combustibles producidos en los procesos de que se trate.

Las razones del rezago relativo pueden enumerarse y hacer una lista muy larga, sin embargo, la realidad poco se conoce. La CONAE (hoy CONUEE) hizo una evaluación del potencial de cogeneración y encontró que éste era muy alto, pero muy disperso. Aunado a esos resultados, las políticas de interconexión y generación distribuida han sido más un freno que una motivación. Las contradictorias políticas energéticas del país han sido la principal causa del fracaso de esos programas. Se tiene la certeza que el rendimiento de las instalaciones de cogeneración está alrededor del 75\% [4]; pudiendo llegar hasta el $85 \%$, razón por la que actualmente, después de la reforma energética, se están fomentando. 
En la Figura 5 se muestra, a manera de ejemplo, cuál es la eficiencia global de la generación de electricidad y calor de forma convencional y en la Figura 6 se muestra la generación de estas mismas magnitudes, pero con un sistema de cogeneración. Se observa que el sistema de cogeneración tiene un $30 \%$ mayor de eficiencia que el sistema convencional [2].

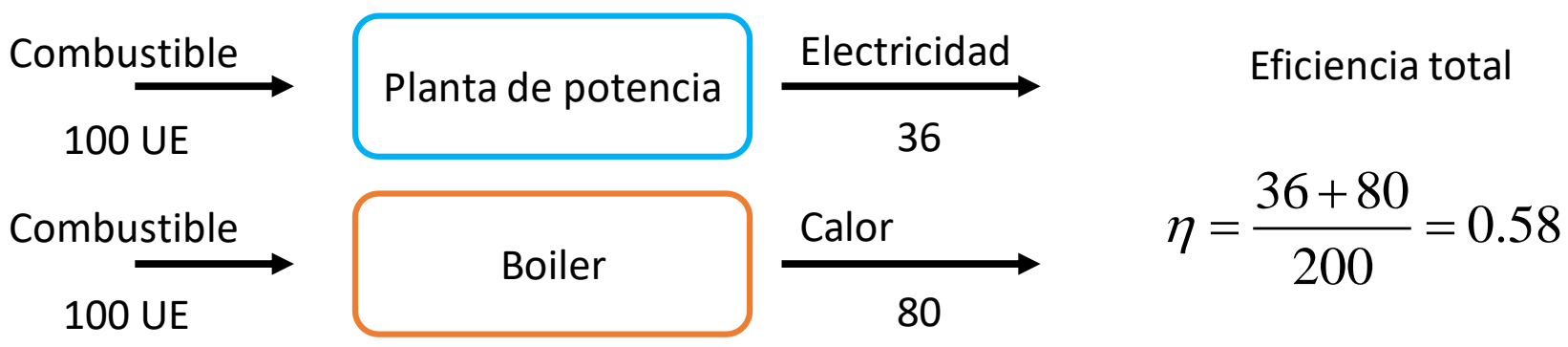

UE: unidades de energía

Figura 5.- Sistema de generación de electricidad y calor convencional.

\section{Eficiencia total}

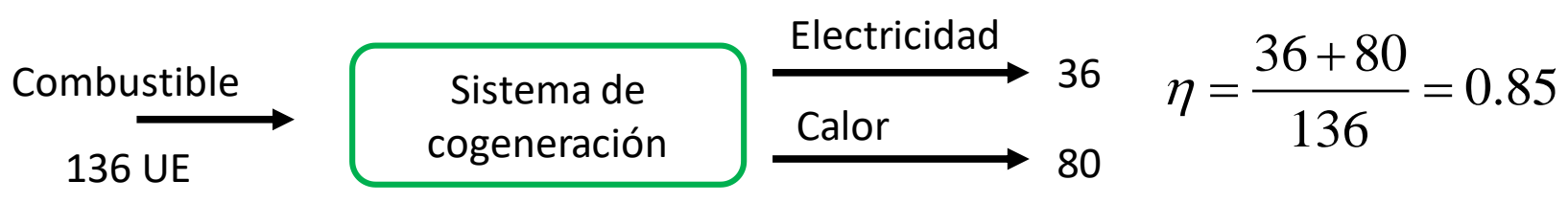

UE: unidades de energía

Figura 6.- Sistema de generación de electricidad y calor con un sistema de cogeneración.

La SENER en 2017 elaboro una prospectiva para el crecimiento de las energías renovables al año 2031 y prevé que la cogeneración eficiente aportará un 5.1\% (Figura 7), sin embargo, este porcentaje puede ser aún mayor al impulsar la micro - cogeneración (MCHP) y atacar también a los sectores de pequeña y mediana industria, que en conjunto son un porcentaje representativo en la reducción de emisiones contaminantes.

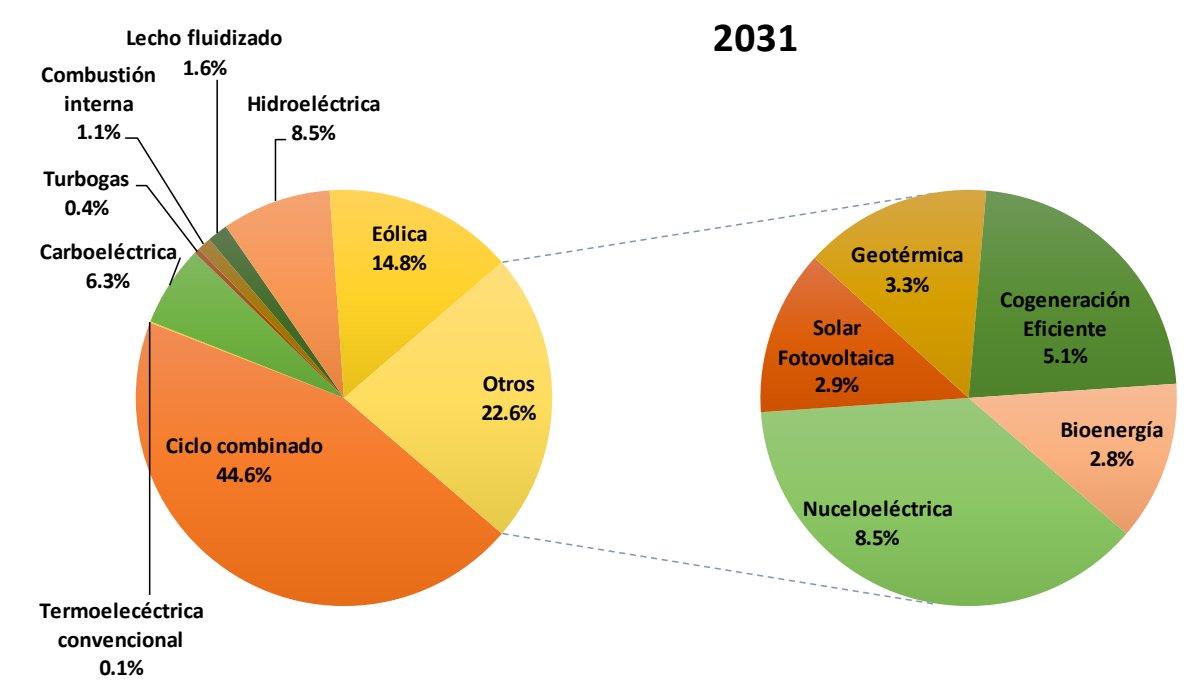

Figura 7.- Prospectiva de la generación de energía al año 2031 
La CHP tiene tres principales aplicaciones:

- Industrial

- Comercial, Institucional y Residencial.

- District Heating and Cooling (DHC)

El sector industrial tiene grandes demandas concurrentes de calor y energía, y tiene la mayor capacidad instalada (en términos de producción de electricidad) de las tres aplicaciones. La calefacción urbana también utiliza los sistemas de cogeneración de manera extensa, lo que proporciona calor para países con largas temporadas de requerimiento de calefacción y cada vez más enfriamiento durante los meses de verano. Sin embargo, los avances en el desarrollo de tecnología han llevado a la disponibilidad de sistemas de cogeneración más pequeños, con costos reducidos, emisiones reducidas y una mayor personalización. Como resultado, los sistemas de CHP se utilizan cada vez más para aplicaciones más pequeñas en los sectores comerciales e institucionales y se incorporan más a menudo en los sistemas de DHC. La Tabla 1 resume estas aplicaciones [20].

Tabla 1.- Aplicaciones de CHP

\begin{tabular}{|c|c|c|c|}
\hline Características & CHP - industrial & $\begin{array}{l}\text { CHP - comercial / } \\
\text { institucional }\end{array}$ & $\begin{array}{c}\text { Calefacción y refrigeración } \\
\text { del distrito }\end{array}$ \\
\hline Clientes típicos & $\begin{array}{l}\text { Productos químicos, pulpa y } \\
\text { papel, metalurgia, } \\
\text { procesamiento pesado } \\
\text { (alimentos, textiles, madera, } \\
\text { minerales), elaboración de } \\
\text { cerveza, hornos de coque, } \\
\text { hornos de vidrio y cerámica, } \\
\text { refinación de petróleo }\end{array}$ & $\begin{array}{l}\text { Fabricación ligera, hoteles, } \\
\text { hospitales, grandes edificios } \\
\text { de oficinas urbanas, } \\
\text { operaciones agrícolas. }\end{array}$ & $\begin{array}{l}\text { Todos los edificios al alcance } \\
\text { de la red de calefacción, } \\
\text { incluidos edificios de } \\
\text { oficinas, casas individuales, } \\
\text { campus, aeropuertos, } \\
\text { industrias }\end{array}$ \\
\hline $\begin{array}{l}\text { Facilidad de } \\
\text { integración con } \\
\text { energías renovables } \\
\text { y residuos } \\
\text { energéticos. }\end{array}$ & $\begin{array}{l}\text { Moderado - Alto } \\
\text { (particularmente flujos de } \\
\text { residuos de energía } \\
\text { industrial) }\end{array}$ & Bajo - moderado & Alto \\
\hline $\begin{array}{l}\text { Nivel de } \\
\text { temperatura }\end{array}$ & Alto & Bajo a medio & Bajo a medio \\
\hline $\begin{array}{l}\text { Tamaño típico del } \\
\text { sistema }\end{array}$ & $1-500 \mathrm{MWe}$ & $1 \mathrm{kWe}-10 \mathrm{MWe}$ & Alguna \\
\hline Primotor & $\begin{array}{l}\text { Turbina de vapor, turbina de } \\
\text { gas, motor alternativo } \\
\text { (encendido por } \\
\text { compresión), ciclo } \\
\text { combinado (sistemas más } \\
\text { grandes) }\end{array}$ & $\begin{array}{l}\text { Motor alternativo } \\
\text { (encendido por chispa), } \\
\text { motores stirling, celdas de } \\
\text { combustible, micro-turbinas. }\end{array}$ & $\begin{array}{l}\text { Turbina de vapor, turbina de } \\
\text { gas, incineración de residuos, } \\
\text { CCGT. }\end{array}$ \\
\hline $\begin{array}{l}\text { Fuente de energía / } \\
\text { combustible }\end{array}$ & $\begin{array}{l}\text { Cualquier combustible } \\
\text { líquido, gaseoso o sólido, } \\
\text { gases residuales de procesos } \\
\text { industriales (por ejemplo, } \\
\text { gases de alto horno, gases } \\
\text { residuales de hornos de } \\
\text { coque, etc.) }\end{array}$ & $\begin{array}{c}\text { Combustibles líquidos o } \\
\text { gaseosos. }\end{array}$ & Cualquier combustible \\
\hline $\begin{array}{l}\text { Patrones de carga de } \\
\text { calor / electricidad }\end{array}$ & $\begin{array}{l}\text { Específico de usuario y } \\
\text { proceso }\end{array}$ & Usuario específico & $\begin{array}{l}\text { Fluctuaciones diarias y } \\
\text { estacionales mitigadas por la } \\
\text { gestión de carga y el } \\
\text { almacenamiento de calor }\end{array}$ \\
\hline
\end{tabular}


En 2011, las instalaciones de cogeneración industrial generaron el 26\% del total de la generación eléctrica mundial a partir de la cogeneración (37\% y 15\% en los países miembros de la Organización para la Cooperación y el Desarrollo Económicos (OCDE) y las economías no miembros, respectivamente) [21].

Actualmente, la cogeneración representa alrededor del 10\% de la generación mundial de electricidad, se estima que para el 2030 haya un crecimiento del 30\% [22]. En la Figura 8 se puede observar que hay cinco países que han ampliado exitosamente el uso de la cogeneración, alrededor del 30-50\% de la generación total de energía: Dinamarca, Finlandia, Rusia, Letonia y los Países Bajos. Cada uno de estos países tiene su propio enfoque, pero su experiencia colectiva demuestra lo que se puede lograr [23].

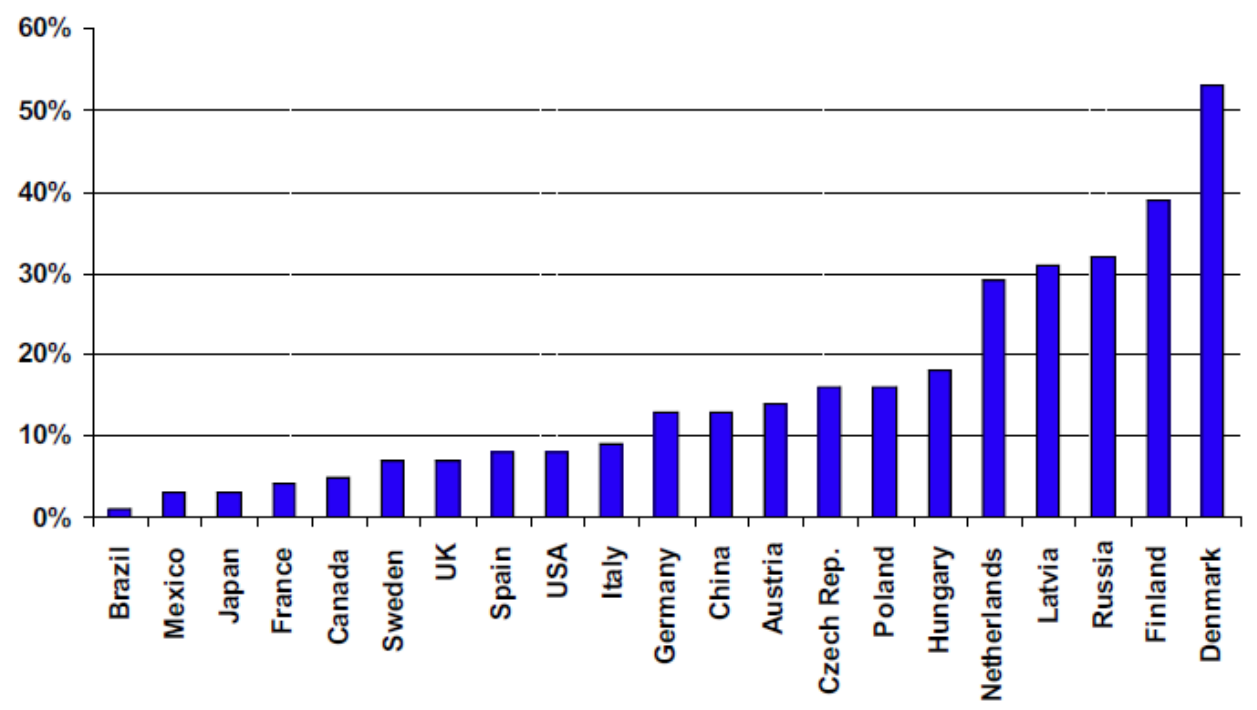

Figura 8.- Participación de la CHP en la producción total de energía nacional.

Para el caso particular de México, en la Figura 9 se muestra la evolución histórica de la capacidad instalada de cogeneración en México según la Comisión Reguladora de Energía (CRE) que tiene registrados en el período de 1994 a 2017 [24]. Se observa que en el año 2015 se otorgaron un gran número de permisos para instalaciones con esta tecnología, esto debido a la reforma energética, la cual impulso este tipo de tecnologías para la mitigación de los GEI. Para el año 2017 ya se tiene un potencial instalado de casi 7,000 MW. Sin embargo, de acuerdo con un estudio realizado por la SENER/CONUEE/GIZ en el 2009, el país puede tener un mayor potencial de generación de energía limpia a través de la cogeneración y se estima que es poco más de 10,000 MW. 


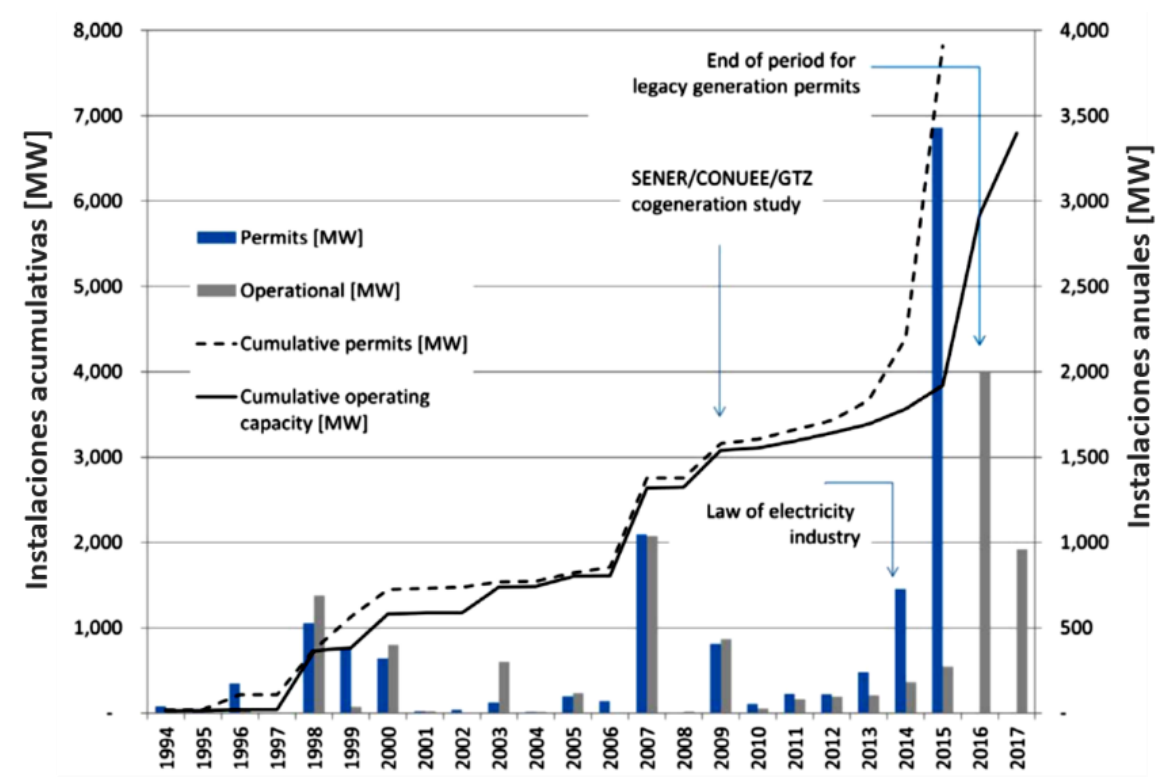

Figura 9.- Evolución histórica de la capacidad instalada de cogeneración en México

Actualmente no existe registro del potencial que pudiera llegar a tener el uso de la MCHP en México. Sin embargo, en otros países si se han implementado estos sistemas teniendo grandes beneficios. Un estudio de GIZ México (2013) reporta que en países como Reino Unido, Alemania, España y U.S.A. se tienen sistemas de MCHP y micro-trigeneración. En 1997 en Alemania se realizó la instalación de un sistema de micro-trigeneración en un centro comercial con una capacidad de 120 kWe, 214 kWth y $135 \mathrm{~kW}$ de refrigeración por absorción. En 2005 en el estado de California USA, se realizó la instalación de un sistema de micro-trigeneración en un hotel con una capacidad de 240 kWe (4 micro-turbinas de 60 kW) y 120 ton de refrigeración. En el 2010 en España se instaló un sistema de MCHP para viviendas con una capacidad de $65 \mathrm{kWe}$ y $120 \mathrm{kWth}$. En 2011 en Reino Unido se instaló un sistema de MCHP para una universidad con una capacidad de 5 kWe y $30 \mathrm{kWth}$. Para ver más detalles de los 4 casos mencionados, ver el anexo A y la Tabla 34 del mismo anexo para ver más casos de estudio de MCHP en el mundo. Todos estos proyectos son de diseño integral, es decir, que son construidos para satisfacer las necesidades eléctricas y térmicas del usuario y los equipos no son de uso comercial, son diseñados específicamente para cada usuario, pero de manera convencional.

El intervalo de potencia para decir que se trata de una MTG varía según el autor; Murugan (2016) considera que una MTG se encuentra en el intervalo de 20-300 kW, mientras que Onovwiona (2006) menciona que es de 30-100 kW y Pilavachi (2002) de 30-150 kW. Como no hay un acuerdo preciso, en este trabajo consideraremos un intervalo de 30-200 kW. Definido el intervalo, la tecnología disponible en el mercado está muy acotada. La MTG más pequeña es de $30 \mathrm{~kW}$. Resource Dynamic Corp (1999) reporta que en el año de 1999 existían 5 fabricantes de MTG: Allied signal, Elliot energy system, Capstone, Power works y ABB. Por otro lado, Giampaolo (2009) reporta que en el año 2003 solo existían 3 fabricantes de MTG: Elliot energy system, Capstone y Power works. Por razones de mercado actualmente solo quedan dos fabricantes: Capstone y Ansaldo Energía.

El calor de escape de una MTG se puede utilizar para diferentes aplicaciones, algunas de ellas son: el calentamiento de agua, producción de vapor, refrigeración por absorción, calefacción, entre otras. Algunas aplicaciones de MTG en CHP no utilizan regeneradores y por consecuencia la temperatura de gases de escape es más alta y habrá mayor calor disponible para la recuperación [14]. 
Las industrias que tienen el mayor potencial de mercado estimado para el uso de MTG en CHP son: alimentos, bebidas, pulpa y papel, textiles, hospitales, hoteles y edificios institucionales.

Normalmente el empleo de los gases de la MTG requiere de una caldera de recuperación de calor o en su caso su inyección directa a un sistema de enfriamiento por absorción. Sin embargo, se pueden hacer propuestas interesantes para reducir la inversión. De esta manera se puede emplear un GV convencional que evitará la inversión de una caldera de recuperación de calor y aprovechar los gases de combustión de la MTG. Para ello es importante realizar un análisis de factibilidad de la implementación de un sistema continuo de aprovechamiento de esos gases debido a que vienen con un elevado exceso de aire y, en principio, no perturbarían el proceso de combustión convencional en el GV. Bajo esta premisa se reduce el consumo de combustible, decrecen sustancialmente las emisiones de GEI y se reducen los costos de inversión y operación. Se debe por lo tanto conocer la magnitud de las alteraciones en los coeficientes de transferencia de calor en el haz de tubos, del lado de la coraza y el coeficiente global. 


\section{Capítulo 2. Pregunta de investigación y objetivos}

\section{Pregunta de investigación}

1) ¿Es compatible el acoplamiento de los gases efluentes de una micro turbina de gas (MTG) a un GV pirotubular pre-existente?

2) ¿El porcentaje de reducción del índice de emisiones de $\mathrm{tCO}_{2} / \mathrm{MWh}$ generado con la implementación del sistema de micro-cogeneración es atractivo para su implementación masiva en el país? ¿Y cómo puede contribuir esto en la meta nacional de reducción de emisiones?

\section{Objetivo general}

Diseñar y evaluar un sistema de micro-cogeneración cuando los gases efluentes de la MTG se inyectan a un GV pirotubular convencional pre-existente.

\section{Objetivos particulares}

1) Analizar la cantidad de energía térmica disponible y el intervalo de temperatura de los gases de combustión de las MTG de 30 y 65 kW con 3 combustibles diferentes.

2) Evaluar la factibilidad de la inyección de los gases de escape de una MTG a un GV pirotubular pre-existente.

3) Evaluar los efectos en el rendimiento térmico del sistema de micro-cogeneración en función del combustible empleado y la temperatura de los gases efluentes de la MTG.

4) Determinar la energía libre de combustible (ELC) de acuerdo con la norma de la CRE (Metodología de cálculo para determinar el porcentaje de energía libre de combustible en fuentes de energía y procesos de generación de energía eléctrica).

5) Cálculo de las emisiones de $\mathrm{CO}_{2}$. 


\section{Capítulo 3. Tecnologías disponibles para Micro-cogeneración (MCHP)}

Las tecnologías disponibles para sistemas de MCHP son: motores reciprocantes, MTG, ciclo Rankine orgánico ORC y celdas de combustible. Cada una de estas tecnologías tiene sus ventajas y desventajas para este tipo de instalaciones. Las más utilizadas son los motores reciprocantes y MTG, esto debido a que son tecnologías que llevan más tiempo de estudio en la mejora de su proceso de producción de energía térmica y eléctrica, las pequeñas celdas de combustible, se encuentran en el mercado desde hace ya algunos años, sin embargo, no se ha extendido su empleo. Las otras tecnologías aún son proyectos pilotos y se siguen realizando pruebas para ser mejoradas. El ciclo Rankine convencional es muy poco frecuentemente empleado por la ausencia de turbinas de baja potencia.

\subsection{Motores reciprocantes}

Los motores reciprocantes se basan en convertir la energía química contenida en un producto combustible en energía térmica y eléctrica (o en su defecto mecánica). Estos son un sistema adecuado para aplicaciones de MCHP debido a su pequeño tamaño y tecnología; sin embargo, necesitan mantenimiento periódico para garantizar la funcionalidad requerida. Están disponibles en el mercado y tienen una amplia gama de tamaños que van desde los $10 \mathrm{~kW}$ a más de $18 \mathrm{MW}$ [25]. En las Figura 10 y 11 se muestran las partes de un motor de combustión interna, donde se observa que tiene muchas partes móviles y por ende tiene mayores pérdidas y se tienen eficiencias de entre el 25-48\% [26].

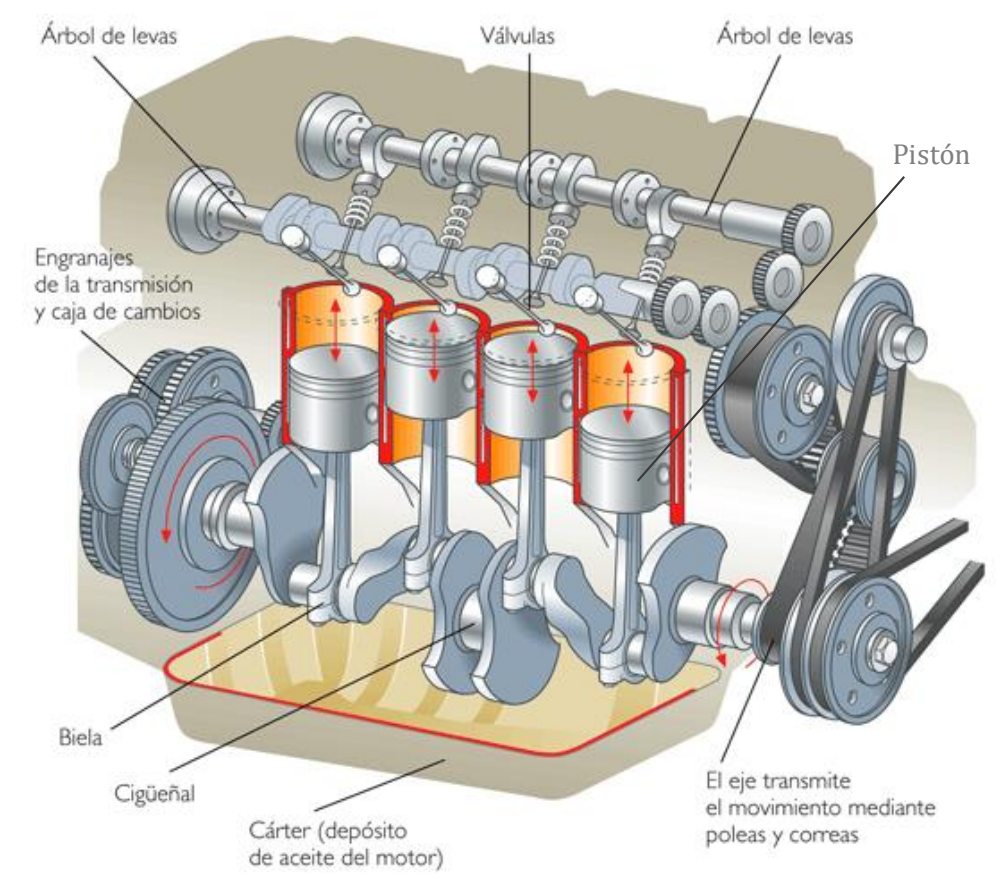

Figura 10.- Diagrama esquemático de un motor de combustión interna

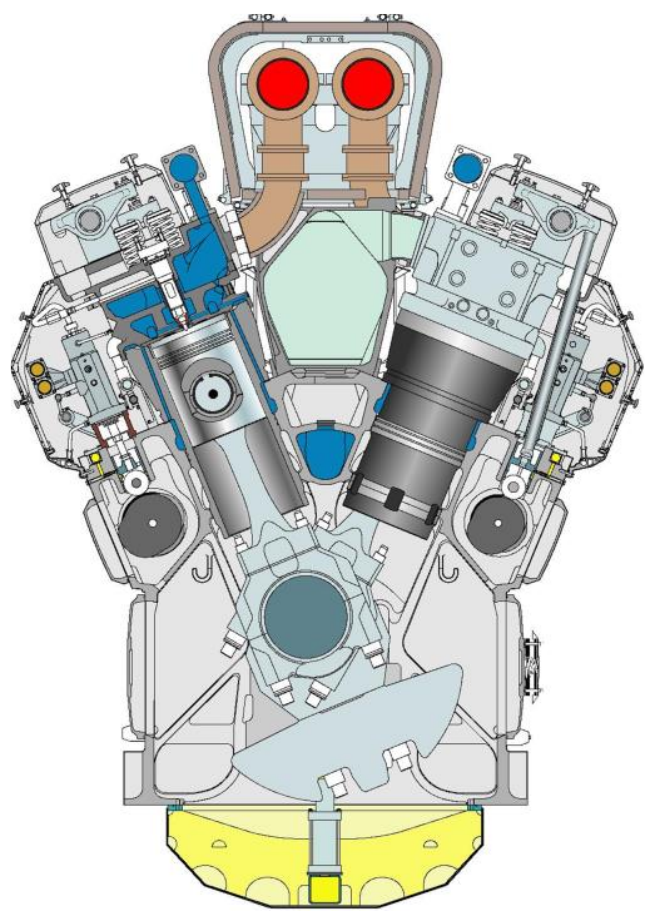

Figura 11.- Motor de combustión interna.

El fin de los intercambiadores de calor es el recuperar la mayor cantidad posible de energía térmica. La recuperación de la energía térmica generada es mediante los circuitos de refrigeración de las camisas, al aceite refrigerante (entre ambos se obtiene el $25 \%$ del total producido) y los gases de escape (65\% del total producido). El $10 \%$ se considera pérdidas. La recuperación se hace normalmente mediante uno o dos circuitos en forma de agua caliente (entrada a $70^{\circ} \mathrm{C}$ y salida a 85 $\left.90^{\circ} \mathrm{C}\right)[26]$. 


\subsection{Micro-turbinas de gas (MTG)}

La diferencia más importante respecto a las turbinas convencionales se sitúa en el hecho de tener un ciclo con regeneración para mejorar el rendimiento total y la ausencia de reductor para conectarse al alternador. Las MTG proporcionan una eficiencia eléctrica cerca del 30\% y una eficiencia térmica entre el 50-60\% [27], tienen la capacidad de ser alimentadas por diferentes tipos de combustibles, los niveles de emisiones son bajos y tienen un gran potencial de recuperación de calor y pueden alcanzar eficiencias del $80 \%$ en cogeneración.

En la Figura 12 se muestran las partes de una MTG y se observa que el rotor de la turbina es su única parte móvil. Tanto el compresor como el alternador son solidarios. Se utilizan cojinetes de aire para evitar el uso de lubricantes y al no utilizarse aceites ni agua de refrigeración, al estar refrigerado por aire, el mantenimiento mecánico es menor y hace que las pérdidas sean menores con respecto a un motor alternativo. La energía térmica generada está contenida en los gases de escape, que salen alrededor de los 270 a $320{ }^{\circ} \mathrm{C}$ [28].

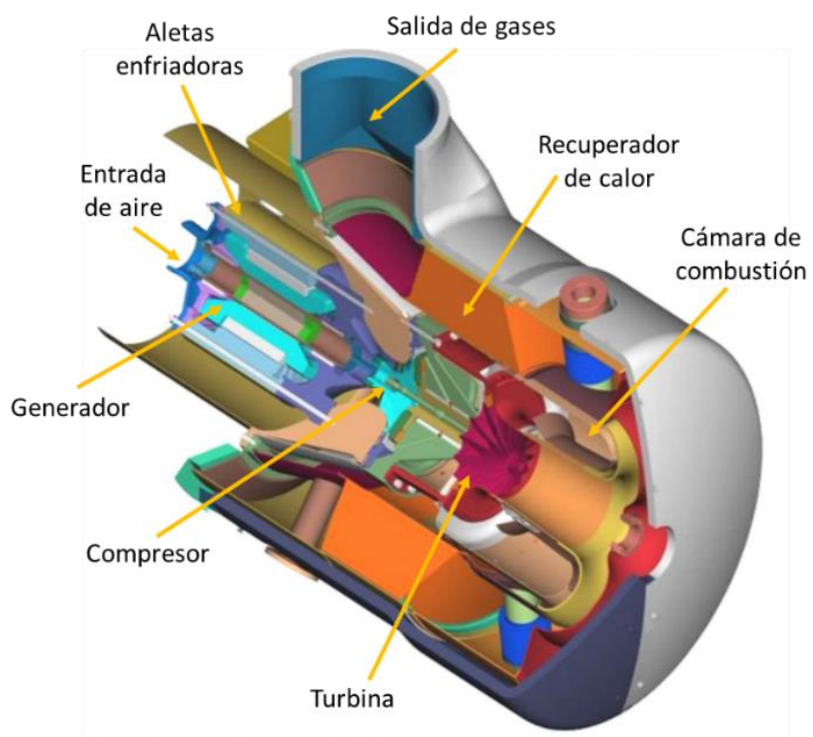

Figura 12.- Diagrama esquemático de las partes de una micro-turbina (Capstone).

\subsection{Ciclo Rankine orgánico ORC}

El esquema de funcionamiento de un ORC es idéntico al de un ciclo de Rankine tradicional como se muestra en la Figura 13, conteniendo una bomba, un GV, una turbina y un condensador. La diferencia fundamental es la sustitución del agua por un fluido orgánico con propiedades de volatilidad superiores al agua como fluido de trabajo. El uso de fluidos orgánicos posibilita el uso de fuentes de calor de temperaturas inferiores [29].

Las diferencias entre el ciclo Rankine convencional y el ORC son las siguientes:

- En el ORC se asegura que no habrá mezcla bifásica a la salida de la turbina (vapor sobrecalentado).

- La entalpia de vaporización de este tipo de fluidos es muy pequeña, ocurriendo casi todo el proceso de aceptación de calor en la zona de estado líquido.

- Hay una amplia gama de fluidos orgánicos cuya presión de condensación a temperatura ambiente es superior a la atmosférica. 


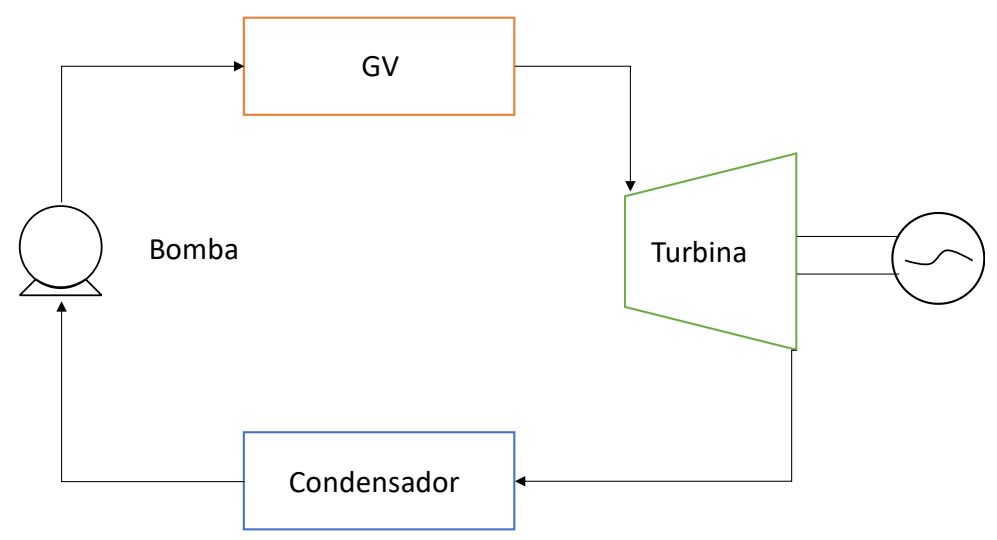

Figura 13.- Diagrama esquemático ciclo ORC

\subsection{Celdas de combustible}

La tecnología de celdas de combustible es una tecnología emergente con un gran potencial para la generación de electricidad para la micro cogeneración con un buen rendimiento y con un daño mínimo al medio ambiente. La diferencia fundamental entre las tecnologías anteriormente descritas y las celdas de combustible es la producción de electricidad, no mediante energía mecánica, sino mediante un proceso de electrolisis inversa.

Las ventajas de los sistemas de micro-cogeneración de celdas de combustible incluyen bajo nivel de ruido, mantenimiento muy bajo, excelente gestión de la carga parcial, emisiones prácticamente nulas y una eficiencia teórica del 85-90\% [4].

Las celdas de combustible pueden ser de varios tipos, aunque las más utilizadas en microcogeneración y las más comercialmente desarrolladas son del tipo PEM (Proton Exchange Membrane) [27] y constituyen las pilas (stacks) para la producción de la energía eléctrica en corriente continua y la producción de calor. El hidrógeno puede provenir de cualquier fuente, como la electrolisis del agua, la reformación de gas natural u otros hidrocarburos, fotoquímica solar, termoquímica solar etc. Se alimenta en el ánodo y el aire que contiene el oxígeno necesario para la reacción, es alimentado en el cátodo (Figura 14). Estas celdas tienen un humidificador que permite transferir el agua para la humidificación directa del vapor en la salida del cátodo que permite una recuperación térmica significativa que aumenta la eficiencia de la celda de combustible.

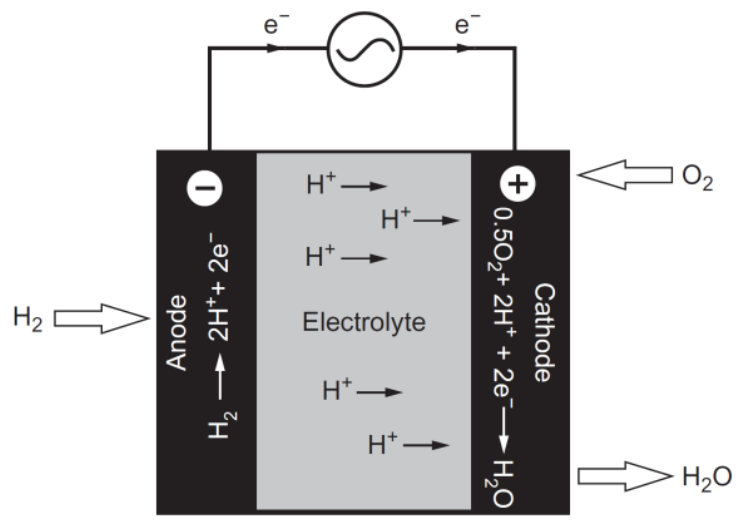

Figura 14.- Principio básico de funcionamiento de una celda de combustible. 


\section{Capítulo 4. Micro-turbina de gas (MTG)}

\subsection{Análisis termodinámico Ciclo Brayton regenerativo}

Las MTG operan bajo un ciclo Brayton regenerativo para mejorar la eficiencia del equipo pre calentando el aire antes de entrar a la cámara de combustión. Las MTG disponibles en el mercado son de paquete integral, es decir, ya vienen integradas con su recuperador de calor (normalmente de placas), su quemador y el generador eléctrico.

En la Figura 15 se muestra el diagrama esquemático de operación de la MTG. Se observa que el aire medio-ambiental es succionado por el compresor y comprimido hasta una presión dada. Éste entra a un recuperador de calor para después entrar a la cámara de combustión donde se mezcla con el combustible utilizado y se efectúa la combustión. Estos gases se expanden en la turbina de gas produciendo un trabajo y finalmente pasan por el recuperador. Una parte del trabajo obtenido se emplea la operación del compresor y la diferencia es convertida en energía eléctrica por medio del generador [30]. La temperatura de entrada de los gases de combustión a la turbina es normalmente entre 800 a $1200^{\circ} \mathrm{C}$ [31][32], a la salida de la turbina la energía térmica de los gases de combustión se utiliza para el precalentado de aire comprimido antes de entrar a la cámara de combustión y la energía térmica remanente se puede emplear para calentar agua o u otras necesidades.

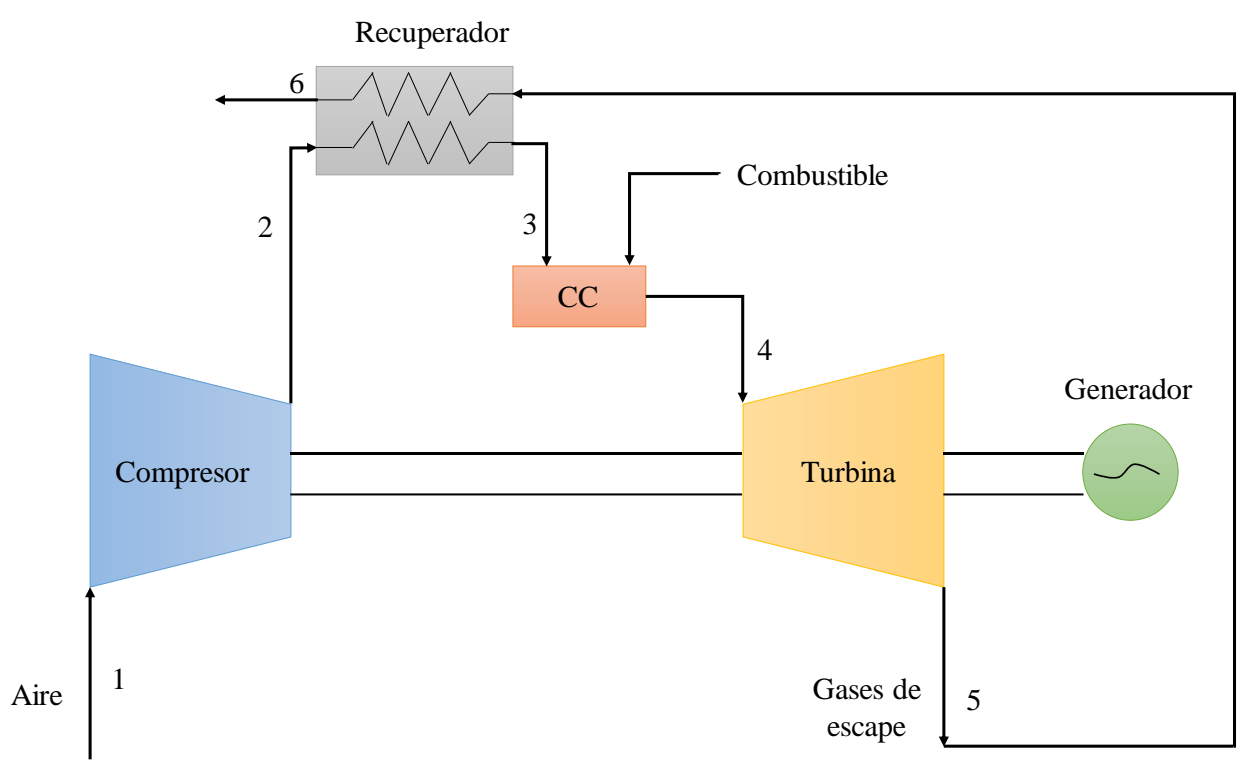

Figura 15.- Diagrama esquemático del ciclo Brayton con regeneración

Las propiedades termodinámicas de las MTG de estudio se determinan bajo las condiciones de referencia de la Internacional Standards Organization (ISO) de $15^{\circ} \mathrm{C}\left(59^{\circ} \mathrm{F}\right), 60 \%$ de humedad relativa y $101.3 \mathrm{kPa}$ (14.7 psia). Debido a esto, el desempeño de las turbinas de gas varía significativamente con las condiciones ambientales locales.

El cálculo de las propiedades termodinámicas se hace de la siguiente manera:

Estado 1.- Admisión de aire al compresor. Se toma como estado de referencia las siguientes condiciones atmosféricas: temperatura $=15^{\circ} \mathrm{C}$ y presión a nivel del mar $=1.013 \mathrm{bar}$. Con esos valores y utilizando la ecuación de gas ideal se obtiene el volumen específico por mol de aire. 


$$
v=\frac{R_{\text {aire }} T}{P}
$$

Para calcular el volumen de aire específico de cada turbina se utiliza el flujo de aire a condiciones ISO, el cual se mantendrá constante en todas las variaciones del presente análisis puesto que la turbina está diseñada para ese volumen específico sin importar las condiciones atmosféricas. Sin embargo, si cambia el flujo másico, como se observará más adelante.

$$
\dot{V}_{\text {aire }}=v_{1} \dot{m}_{\text {aireISO }}
$$

Estado 2.- Salida del aire del compresor. Con base en la relación de presiones $\pi$ se obtiene $\mathrm{P}_{2}$ y a partir de la eficiencia del compresor que se considera en primera instancia de $\eta_{c}=0.8$ se obtiene la $T_{2}$. Sin embargo, el fabricante, en su información, no proporciona la $\pi$, por tal razón se tiene que determinar de forma iterativa y de acuerdo a lo encontrado en la literatura estas pueden operar en un intervalo de $\pi$ igual a 1.5 hasta 7 [33][34], Por ello $\pi$ se iteró en ese intervalo.

$$
\begin{gathered}
T_{2}=T_{1}\left[1+\frac{1}{\eta_{s i c}}\left(\pi^{x}-1\right)\right] \\
x=\frac{\gamma-1}{\gamma}
\end{gathered}
$$

Donde: $\quad x=\frac{\gamma-1}{\gamma}$

Una vez conocido $\mathrm{T}_{2} \mathrm{Y} \mathrm{P}_{2}$ se puede obtener el volumen específico v2 usando (1).

Estado 4.- Entrada de los gases de combustión a la turbina. El fabricante no proporciona la temperatura a la entrada de los gases. Para obtener esta temperatura se buscó en la literatura el rango de temperatura en el cual operan estas turbinas para así poder acotar las iteraciones, encontrando un intervalo que va de 700 a $1300^{\circ} \mathrm{C}$ [35][36]. Además, considerando que no hay caídas de presión en el regenerador y en la cámara de combustión $\mathrm{P}_{4}=\mathrm{P}_{2}$, conocidas $\mathrm{T}_{4}$ y $\mathrm{P}_{4}$ usando (12) se obtiene $\mathrm{v}_{4}$.

Estado 5s.- Salida de la turbina isoentrópico. Considerando la expansión isoentrópica de la turbina se obtiene $\mathrm{T}_{5 \mathrm{~s}}$ con la siguiente ecuación:

$$
T_{5 s}=T_{4}\left(\frac{P_{3}}{P_{4}}\right)^{x}
$$

$\begin{array}{ll} & P_{4}=P_{3}=P_{2} \\ \text { Considerando: } & P_{5}=P_{6}=P_{1}\end{array}$

$$
T_{5 s}=T_{4}\left(\frac{1}{\pi^{x}}\right)
$$

Estado 5.- Salida de la turbina real. A partir de la eficiencia de la turbina se obtiene $\mathrm{T}_{5}$ :

$$
\eta_{s i t}=\frac{T_{4}-T_{5}}{T_{4}-T_{5 s}}
$$




$$
T_{5}=T_{4}-\eta_{s i t}\left(T_{4}-T_{5 s}\right)
$$

Estado 3.- Entrada a la cámara de combustión. La temperatura del aire a la entrada de la cámara de combustión se obtiene a partir de la definición de la eficacia del regenerador [37]:

$$
\begin{gathered}
E=\frac{T_{\mathrm{c}, \mathrm{sal}}-T_{\mathrm{c}, \text { ent }}}{T_{h, \text { ent }}-T_{c, e n t}} \\
E=\frac{T_{3}-T_{2}}{T_{5}-T_{2}} \\
T_{3}=T_{2}+E\left(T_{5}-T_{2}\right)
\end{gathered}
$$

Estado 6.- Salida del regenerador de la corriente caliente. La temperatura $\mathrm{T}_{6}$, que descargan el regenerador a proceso, se obtiene a partir de un balance de materia y energía como se muestra a continuación [38]:

$$
\begin{aligned}
& \dot{m} C p\left(T_{5}-T_{2}\right)=\dot{m} C p\left(T_{3}-T_{6}\right) \\
& T_{6}=T_{5}-\left(T_{3}-T_{2}\right)
\end{aligned}
$$

También se puede obtener a partir de la eficacia del regenerador:

$$
T_{6}=T_{5}-E\left(T_{5}-T_{2}\right)
$$

\subsection{Diseño del sistema de micro-cogeneración (MCHP) con turbina de gas}

En los sistemas de CHP normalmente se diseña e invierte en un intercambiador para la recuperación de calor para el aprovechamiento de la energía en los gases de combustión. En el diseño de un sistema de MCHP se analizan diversas alternativas para la recuperación de esa energía, sin embargo, se ha requerido del diseño e inversión de ese dispositivo. En este trabajo se hace la propuesta de utilizar un equipo pre-existente, que evitará la inversión de una caldera de recuperación de calor o bien de un intercambiador de calor y así aprovechar la energía que traen los gases efluentes de una MTG. Sin embargo, se deben tener en consideración varios aspectos, como son: las propiedades termo-físicas de los gases, materiales del GV pirotubular pre-existente, diámetros de tuberías, regímenes de operación, entre otras.

Se debe realizar, por consecuencia, un estudio de viabilidad técnica-teórica del uso de esos gases efluentes de la MTG en un GV pirotubular convencional. Es importante conocer las condiciones de operación del GV pre-existente para poder elegir la MTG a acoplar, debido a las altas temperaturas con que salen los gases de escape $\left(270\right.$ a $\left.320{ }^{\circ} \mathrm{C}\right)$ y ver si con el flujo másico de gases con que se cuenta es suficiente para el GV, en caso contrario estimar la cantidad de combustible y aire extra necesarios para alcanzar las condiciones de operación o viceversa; analizar si los gases efluentes de la turbina que cubre las necesidades eléctricas cubre los requerimientos del GV pre-existente.

Para poder hacer este análisis, se necesita conocer el funcionamiento del GV de forma convencional, conocer la cantidad y calidad de los gases provenientes de las MTG's y finalmente hacer el acoplamiento de estos a el GV. En este trabajo se estudió una gama de GV's pirotubulares de 
la marca Cleaver Brooks. Se analizó esta marca debido a que otras marcas no proporcionaban datos técnicos necesarios para hacer el estudio, y de suerte que la simulación fuera lo más cercano a las condiciones de operación reales. El análisis se realizó sin tomar en cuenta la variación de carga en el GV.

Debido a que en el país no se cuenta con una distribución homogénea de GN como se muestra en la Figura 16, se realizó el estudio empleando además GLP e hidrógeno, con la finalidad de mostrar los beneficios de un sistema de MCHP aún si no se cuenta con suministro de GN, combustible con el que normalmente operan las MTG's y tienen una mejor eficiencia.
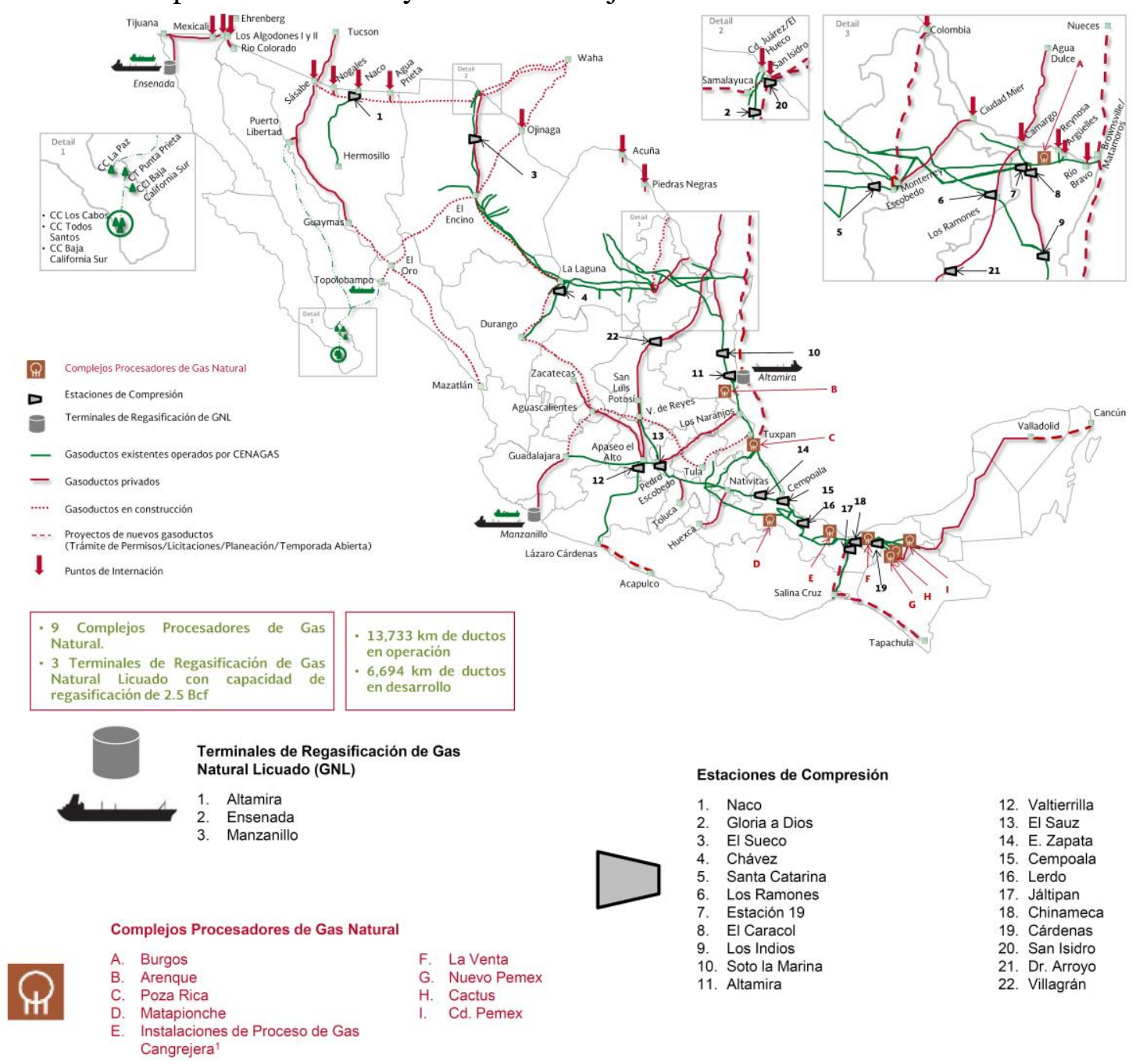

Notas:

1. Instalaciones de Proceso Gas Cangrejera (anteriormente denominado Área Coatzacoalcos) se compone de plantas y equipos de procesos distribuidos en los complejos: (i) Morelos, (ii) Pajaritos, y (iii) Cangrejera (propiedad de Pemex Transformación Industrial (TRI)).

2. Se indican como gasoductos en desarrollo a los gasoductos: (i) en fase de desarrollo de proyecto, (ii) trámite de permiso, (iii) en construcción o (iv) desarrollo de proyecto, (ii) tramite de permiso, (iii) en construccion o (iv)
proceso de temporada abierta.

3. El proyecto de suministro de gas natural a la peninsula de Baja California considera que el transportista recibirá el gas natural en algún punto del territorio nacional, lo transportará por vía marítima y lo entregará en las centrales de generación de la CFE, localizadas en la península de Baja California Sur. El transportista podrá escoger la tecnología más adecuada (convertir, regasificar y transportar por vía terrestre hasta los puntos de

Estaciones de Compresión
$\begin{array}{ll}\text { 1. Naco } & \\ \text { 2. Gloria a Dios } & \text { 12. Valtierrilla } \\ \text { 3. El Sueco } & \text { 13. El Sauz } \\ \text { 4. Chávez } & \text { 14. E. Zapata } \\ \text { 5. Santa Catarina } & \text { 15. Cempoala } \\ \text { 6. Los Ramones } & \text { 16. Lerdo } \\ \text { 7. Estación } 19 & \text { 17. Jáltipan } \\ \text { 8. El Caracol } & \text { 18. Chinameca } \\ \text { 9. Los Indios } & \text { 19. Cárdenas } \\ \text { 10. Soto la Marina } & \text { 20. San Isidro } \\ \text { 11. Altamira } & \text { 21. Dr. Arroyo } \\ \end{array}$
entrega).

Figura 16.- Infraestructura nacional de gas natural 2017 [39]. 


\section{Capítulo 5. Generador de vapor convencional}

Una caldera es el nombre que comúnmente se le da en México a un GV. Este es un equipo diseñado para transferir calor producido por combustión, o mediante electricidad, a un fluido determinado, es decir, genera y entrega vapor en la cantidad, calidad y oportunidad requeridas por el usuario final con operación económica y segura, entendiéndose la calidad del fluido por la presión, temperatura, humedad, pureza y continuidad en la generación y dentro de las tolerancias que el uso final requiera [40].

Los GV's, exceptuando los eléctricos, tienen 6 componentes básicos:

1. Quemador: Aporta el combustible y el aire de combustión, los mezcla y produce la combustión.

2. Cámara de combustión: También llamado hogar, es el espacio donde se aloja la llama, es decir, se produce la combustión y se transfiere calor por radiación.

3. Sección de convección: Zona donde se transfiere el calor de los gases de combustión al fluido a través de las superficies de los tubos.

4. Chimenea: Por donde salen los gases de combustión después de transferir el calor al fluido.

5. Soplador de aire: Proporciona el aire de combustión y lo impulsa a través del GV.

6. Instrumentación y controles: Permiten efectuar la operación con la mayor seguridad y alcanzar mayores niveles de eficiencia.

Los GV's se clasifican por diferentes criterios relacionados con la disposición de los fluidos y su circulación, el mecanismo de transmisión de calor dominante, el tipo de combustible empleado, la presión de trabajo, el tiro, el modo de operación y parámetros exteriores a el GV ligados a la implementación, ubicación, lugar de montaje y aspectos estructurales.

Algunas de las clasificaciones de los GV's se muestran en la Figura 17 según los criterios siguientes:
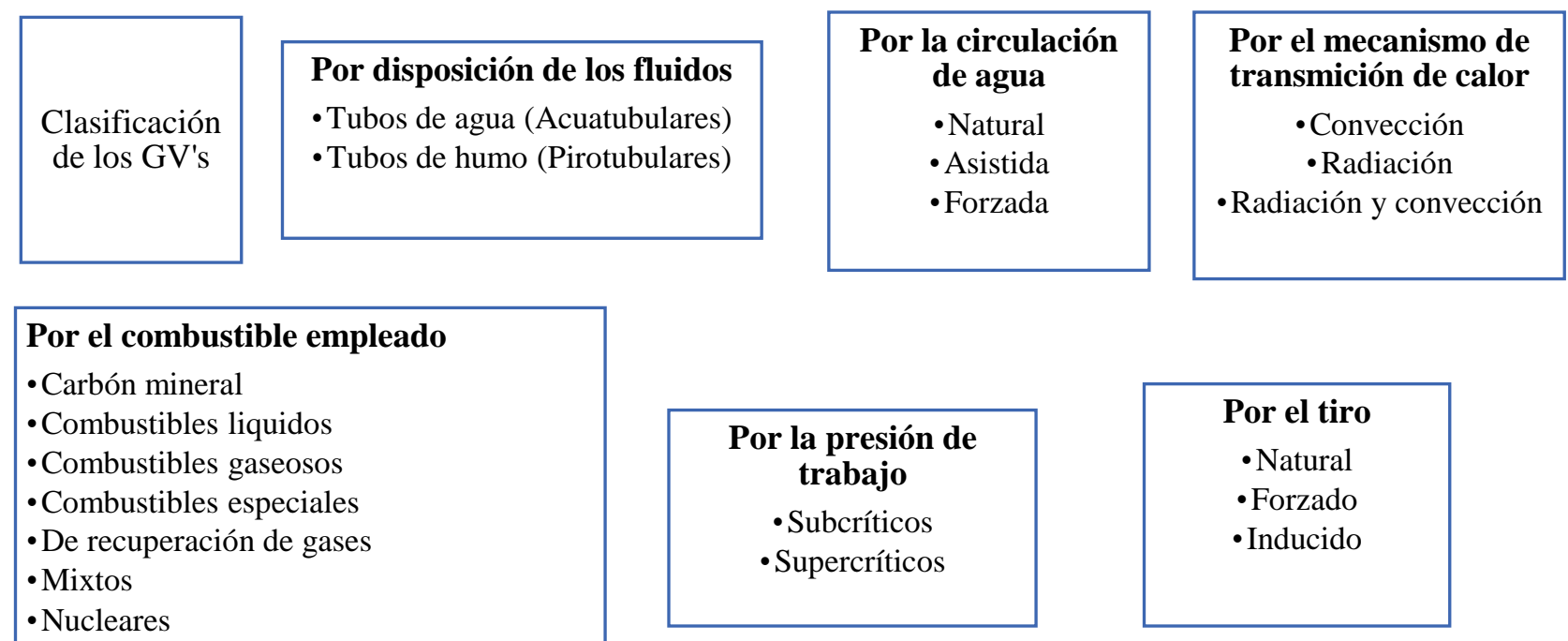
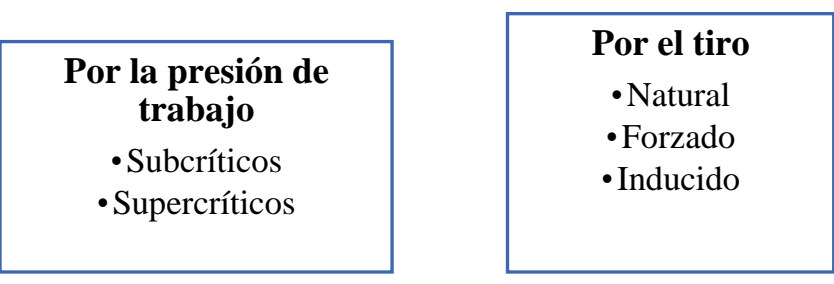

Figura 17.- Clasificación de los GV's. 
Los GV's más utilizados en la pequeña y mediana industria son por disposición de los fluidos, y en particular los de tipo tubos de humo, donde los gases calientes fluyen por el interior de los tubos que son sumergidos en agua dentro de un casco como se muestra en la Figura 18. Las presiones operativas de diseño son próximas a 10.34 bar (150 psig) y sus capacidades varían entre 7.45 a 596.55 kW (10 y $800 \mathrm{hp})$, equivalentes a producciones de vapor de 156.48 a $12519.14 \mathrm{~kg} / \mathrm{h}$ (345 a 27,600 $\mathrm{lb} / \mathrm{hr})[40]$.

Este tipo de GV son apropiados para ser empleados en sistemas de MCHP, ya que no operan bajo grandes presiones y es precisamente lo que se busca. En la Tabla 2 se muestran las ventajas y desventajas del empleo de este tipo de GV [41].

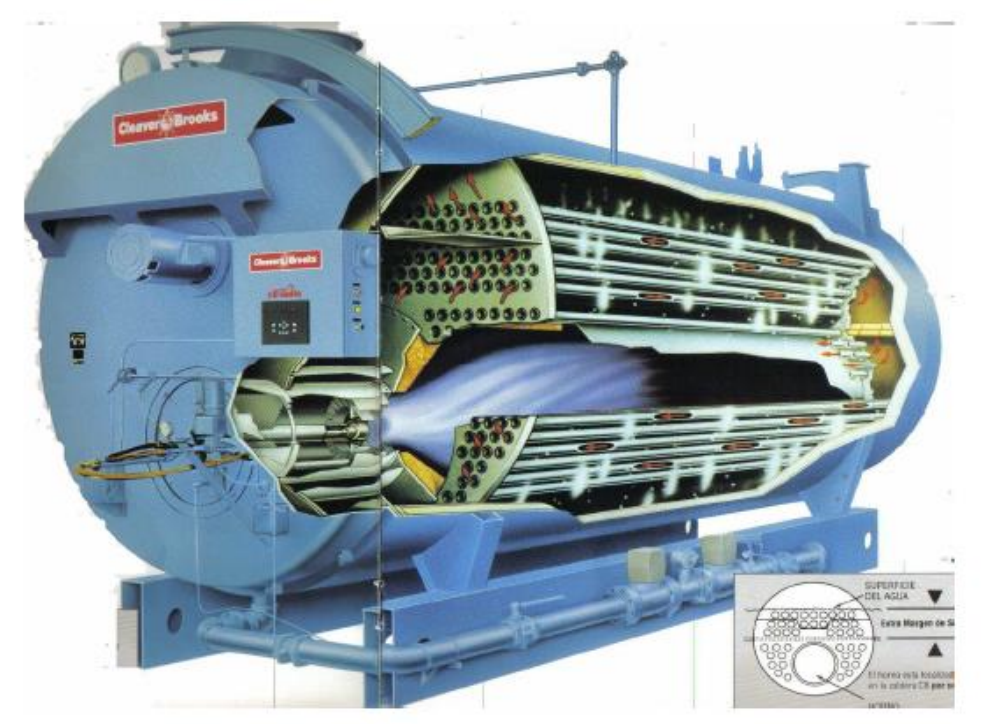

Figura 18.- Generador de vapor tubos de humo.

Tabla 2.- Ventajas y desventajas de los GV's tubo de humo.

\begin{tabular}{|c|c|}
\hline Ventajas & Desventajas \\
\hline $\begin{array}{l}\text { - Requieren bajo costo de inversión y son } \\
\text { menos costosos que los de tubos de agua. } \\
\text { - Alcanzan elevadas eficiencias }(>80 \%) \text {. } \\
\text { - Pueden absorber grandes y súbitas } \\
\text { fluctuaciones de carga con ligeras } \\
\text { variaciones de presión debido al gran } \\
\text { volumen de agua contenido en el casco. }\end{array}$ & $\begin{array}{l}\text { - Mayor tiempo para subir presión y entrar en } \\
\text { funcionamiento. } \\
\text { - Gran peligro en caso de exposición o } \\
\text { ruptura, debido al gran volumen de agua } \\
\text { almacenado. } \\
\text { - No son empleadas para altas presiones. }\end{array}$ \\
\hline
\end{tabular}

\subsection{Características principales}

\section{$\underline{\text { Potencia térmica }}$}

- La potencia térmica nominal, es la máxima energía que se puede aportar en el hogar del GV, por unidad de tiempo

- La potencia térmica útil, es la máxima energía que puede absorber el fluido caloportador en el $\mathrm{GV}$, por unidad de tiempo.

La diferencia entre ambos valores es función de las pérdidas de energía en el GV, por radiación, conducción y convección al ambiente que rodean al GV y por calor de los gases de combustión que se envían a la atmósfera. 


\section{Superficie de calefacción}

Es la superficie de intercambio de calor en contacto con el fluido caloportador. La superficie de radiación es la zona de contacto con la llama y la superficie de convección es la que se encuentra en contacto con los gases de combustión.

\section{Presión}

- Presión de diseño es la utilizada como base de cálculo al establecer la resistencia de los elementos constituyentes del GV.

- Presión de servicio, es la presión a la cual trabajara in situ.

\section{Patrones de flujo de gas}

- Dry back. - Este arreglo puede ser de 2, 3 y 4 pasos, dry back se refiere a que el último paso es en seco, no hay agua, antes de salir a la chimenea como se muestra en las Figuras 19, 20 y 21.

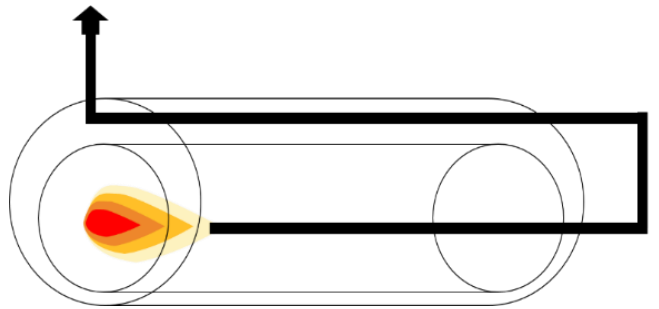

Figura 19.- Diagrama esquemático GV de 2 pasos DryBack

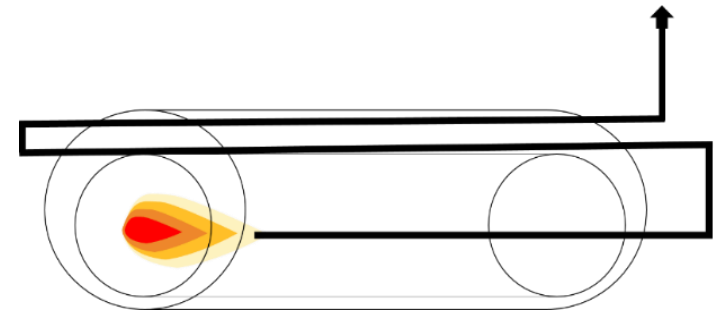

Figura 20.- Diagrama esquemático GV de 3 pasos DryBack

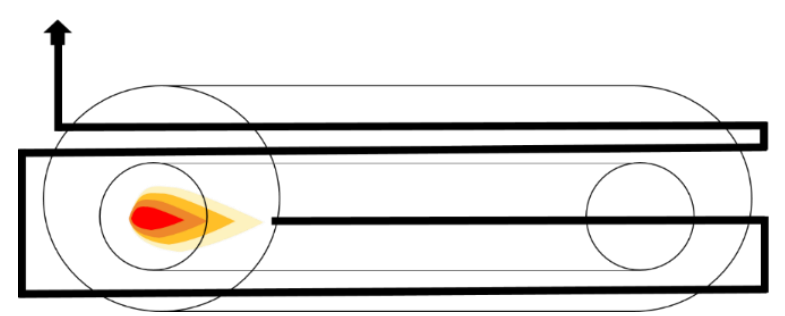

Figura 21.- Diagrama esquemático GV de 4 pasos Dry-Back

- Wet back. - Este arreglo pude ser de 3 o 4 pasos y el hogar se encuentra en el centro del GV para poder tener paso de tubos alrededor de ella como se muestra en las Figuras 22 y 23 , donde el último paso antes de salir a la chimenea está sumergida en agua.

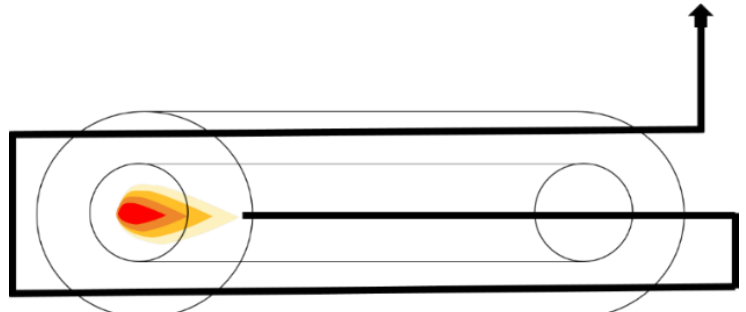

Figura 22.- Diagrama esquemático $G V$ de 3 pasos Wet-Back

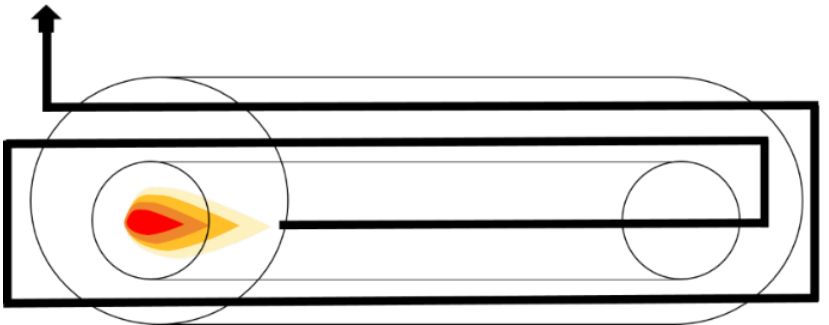

Figura 23.- Diagrama esquemático GV de 4 pasos Wet-Back 


\section{Exceso de oxigeno}

$\mathrm{El}$ porcentaje de $\mathrm{O}_{2}$ registrado en un instrumento equivale al porcentaje de exceso de aire. Por ejemplo, $3 \%$ de $\mathrm{O}_{2}$ es aproximadamente $15 \%$ de exceso de aire y $4 \%$ de $\mathrm{O}_{2}$ es aproximadamente $20 \%$ de exceso de aire. El porcentaje exacto de exceso de aire es un cálculo matemático basado en un análisis de combustible final del combustible que se está quemando. Generalmente se recomienda que las lecturas de $\mathrm{O}_{2}$ estén entre $2 \%$ a $4 \%$, en función del combustible, y se tengan menos de 400 ppm CO, en fuego alto, de acuerdo con la NORMA Oficial Mexicana NOM-085-SEMARNAT-2011, Contaminación atmosférica-Niveles máximos permisibles de emisión de los equipos de combustión de calentamiento indirecto y su medición [42].

En fuego bajo, requiere de mayor exceso de aire y la lectura de $\mathrm{O}_{2}$ de gas de chimenea debe estar entre 6-7\%. Sin embargo, con un quemador de última generación y un mejor control del proceso, el exceso de aire deberá permanecer constante en función del factor de carga del GV.

\section{Zonas de transferencia de calor}

- Transferencia de calor por convección desde los gases de combustión hacia la pared del tubo.

- Transferencia de calor por conducción desde la superficie interna del tubo hasta la externa.

- Transferencia de calor por convección desde la superficie exterior del tubo hacia el agua. 


\section{Capítulo 6. Metodología}

\subsection{Analizar la cantidad y calidad de energía térmica disponible de las MTG de 30 y $65 \mathrm{~kW}$ con 3 combustibles diferentes.}

Las MTG son maquinarias capaces de generar potencia mecánica a partir de la combustión de una mezcla de combustible y aire en determinadas condiciones de presión y temperatura [31][43]. Las condiciones ambientales juegan un papel importante en esa potencia generada, de ahí que éstas se variaron para obtener el coeficiente de derrateo para cada MTG. La presión como función de la altura SNM se varió de 0 a $3500 \mathrm{msnm}$, con intervalos de $500 \mathrm{~m}$ y para la temperatura de 0 a $50^{\circ} \mathrm{C}$, con intervalos de $5^{\circ} \mathrm{C}$. Estos intervalos se tomaron acorde a las condiciones extremas que se pueden tener a lo largo del país.

Las características de las turbinas por analizar son las mostradas en la Tabla 3, que muestran las especificaciones proporcionados por el fabricante [28] [44]. En el Anexo B se muestran las fichas técnicas.

Tabla 3.- Especificaciones de las micro-turbinas de estudio.

\begin{tabular}{|c|c|c|}
\hline Modelo & $\mathbf{C 3 0}$ & C65 \\
\hline \multicolumn{3}{|l|}{ Desempeño eléctrico } \\
\hline Potencia nominal de salida [kW] & 30 & 65 \\
\hline Tensión [VAC] & 400 a 480 & 400 a 480 \\
\hline Tipo & Trifásico & Trifásico \\
\hline \multicolumn{3}{|l|}{ Frecuencia $[\mathrm{Hz}]$} \\
\hline conectado a la red & $50 / 60$ & $50 / 60$ \\
\hline modo aislado & $10-60$ & $10-60$ \\
\hline \multicolumn{3}{|l|}{ Corriente máxima de salida [A] } \\
\hline conectada a la red & 46 & 100 \\
\hline modo aislado & 54 & 127 \\
\hline Rendimiento eléctrico [\%] & 26 & 29 \\
\hline \multicolumn{3}{|l|}{ Características del combustible } \\
\hline Presión de entrada [bar] & 3.79 a 4.14 & 5.17 a 5.52 \\
\hline Flujo de combustible HHV [MJ/h] & 457 & 888 \\
\hline CTU $[k J / k W h]$ & 13800 & 12400 \\
\hline \multicolumn{3}{|l|}{ Emisiones } \\
\hline NOx@15\% $\mathrm{O}_{2}$ ppmvd & 9 & 9 \\
\hline Caudal de gases de escape [kg/s] & 0.31 & 0.49 \\
\hline Temperatura salida de gases $\left[{ }^{\circ} \mathrm{C}\right]$ & 275 & 309 \\
\hline Calor disponible a $70{ }^{\circ} \mathrm{C}[\mathrm{kW}]$ & 65 & 105 \\
\hline
\end{tabular}

El análisis de estas MTG's se realizará usando como combustible GN, GLP e $\mathrm{H}_{2}$, con las composiciones mostradas en la Tabla 4 obteniéndose un PCI de 55,090, 46,024 y 120,011 kJ/kg respectivamente [45] [46].

\begin{tabular}{lc}
\hline \multicolumn{2}{c}{ Gas Natural } \\
\hline Compuesto & Porcentaje [\%] \\
\hline $\mathrm{CH}_{4}$ & 94 \\
$\mathrm{C}_{2} \mathrm{H}_{6}$ & 3 \\
$\mathrm{CO}_{2}$ & 1 \\
$\mathbf{N}_{2}$ & 1 \\
$\mathrm{C}_{3} \mathrm{H}_{8}$ & 0.5 \\
$\mathbf{C}_{4} \mathrm{H}_{10}$ & 0.3 \\
$\mathbf{C}_{5} \mathrm{H}_{12}$ & 0.2 \\
\hline
\end{tabular}

Tabla 4.- Composición de cada uno de los combustibles

\begin{tabular}{|c|c|c|c|}
\hline \multicolumn{2}{|c|}{ Gas LP } & \multicolumn{2}{|c|}{ Hidrógeno } \\
\hline Compuesto & Porcentaje [\%] & Compuesto & Porcentaje [\%] \\
\hline $\mathrm{C}_{3} \mathrm{H}_{8}$ & 30 & $\mathrm{H}_{2}$ & 100 \\
\hline $\mathrm{C}_{4} \mathrm{H}_{10}$ & 70 & & \\
\hline
\end{tabular}


Para cada uno de los combustibles es necesario conocer la temperatura de flama adiabática y analizar su comportamiento en función del exceso de aire. La temperatura de los gases a la entrada de la MTG, dependen de los materiales de su diseño. De esta manera, se selecciona el exceso de aire para abatir la temperatura de flama adiabática hacia las condiciones de diseño. En la Figura 24 se muestra el comportamiento de la temperatura de flama adiabática en función del exceso de aire para 3 combustibles diferentes. Cabe resaltar el caso del hidrógeno cuya temperatura de flama es sensiblemente más alta para el mismo exceso de aire de los otros combustibles.

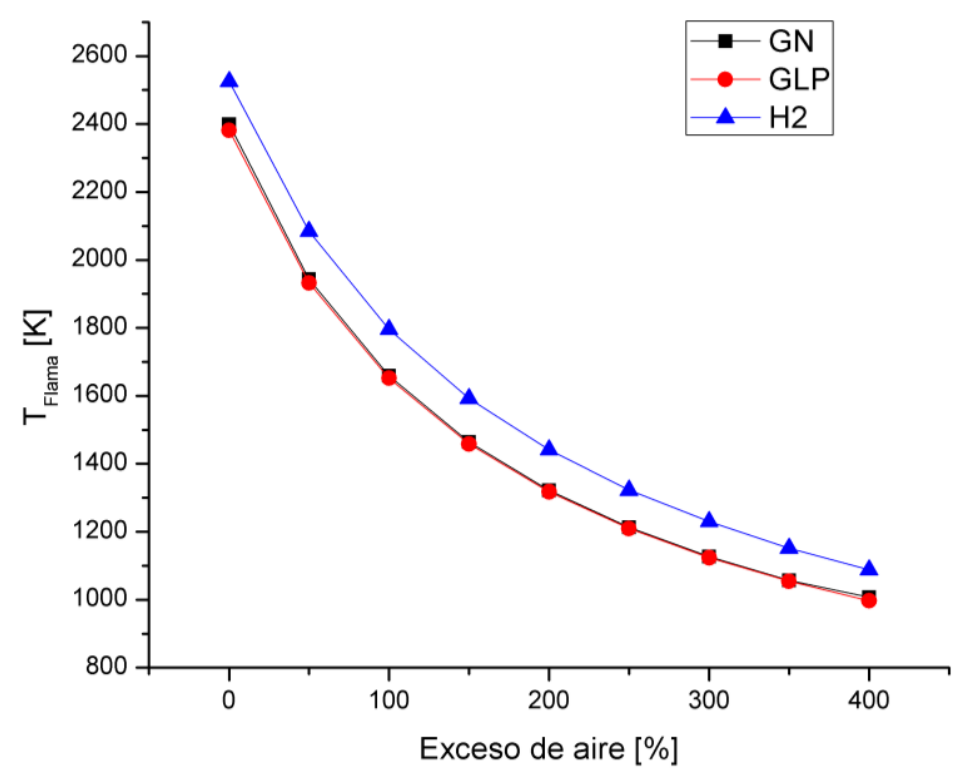

Figura 24.- Temperatura de flama adiabática en función del exceso de aire

\subsubsection{Gas natural y gas LP}

La metodología se realizó utilizando como referencia la composición del gas natural y de forma análoga se puede aplicar la metodología para el gas Lp.

Con los datos del fabricante de cada turbina gas se calculó el flujo de combustible con la siguiente ecuación:

$$
\begin{aligned}
& E_{c}=\frac{\dot{W}}{\eta_{\text {elec }}} \\
& E_{c}=\dot{m}_{\text {comb }} P C I \\
& \dot{m}_{\text {comb }}=\frac{E_{c}}{P C I}
\end{aligned}
$$

Una vez obtenido éste, se determina el flujo de aire a partir del caudal de los gases de escape:

$$
\dot{m}_{\text {aire }}=\dot{m}_{g c}-\dot{m}_{\text {comb }}
$$

Una vez conocido ambos flujos se puede conocer la $R A C_{\text {real }}$ como se muestra en (5). 


$$
R A C_{\text {real }}=\frac{\dot{m}_{\text {aire }}}{\dot{m}_{\text {comb }}}
$$

Para obtener la $R A C_{s t}$ se utiliza la ecuación de combustión en base a la composición del gas natural empleado como se mostró en la Tabla 3.

$$
C_{n} H_{m}+(1+x)\left(n+\frac{m}{4}\right)\left(O_{2}+3.76 N_{2}\right) \rightarrow n C O_{2}+\frac{m}{2} H_{2} O+x\left(n+\frac{m}{4}\right) O_{2}+(1+x)\left(n+\frac{m}{4}\right) 3.76 N_{2}
$$

Donde n y m se obtienen a partir de la composición del gas natural. En la Tabla 5 se muestran

\begin{tabular}{|c|c|c|}
\hline \multicolumn{3}{|c|}{ Tabla 5.- Calculo de $n$ y $m$} \\
\hline & $\mathrm{C}$ & $\mathrm{H}$ \\
\hline & 0.94 & 3.76 \\
\hline & 0.06 & 0.18 \\
\hline & 0.015 & 0.040 \\
\hline & 0.012 & 0.03 \\
\hline & 0.010 & 0.024 \\
\hline Total & 1.037 & 4.034 \\
\hline
\end{tabular}
los cálculos obtenidos para obtener la composición.

Con lo anterior se tiene la forma compacta siguiente:

$$
C_{1.037} H_{4.034}
$$

Donde su masa molecular es:

$$
M M=12 n+m
$$

Una vez conocido lo anterior la $R A C_{s t}$ se calcula de la siguiente manera:

$$
R A C_{s t}=\frac{\left(n+\frac{m}{4}\right) * 4.76 * 29}{M M}
$$

La $R A C_{\text {real }}$ también se puede calcular de la siguiente manera:

$$
R A C_{\text {real }}=(1+x)\left(R A C_{s t}\right)
$$

A partir de la ecuación anterior se obtiene el exceso de aire, despejando x, queda de la siguiente manera:

$$
x=\frac{R A C_{r e a l}}{R A C_{s t}}-1
$$

Una vez conocidas las condiciones ISO se procede al cálculo de los estados termodinámicos del ciclo de una MTG la cual operando bajo un ciclo Brayton regenerativo como se muestra en la Figura 25, a partir de ello se calculan sus estados termodinámicos como se muestra a continuación: 


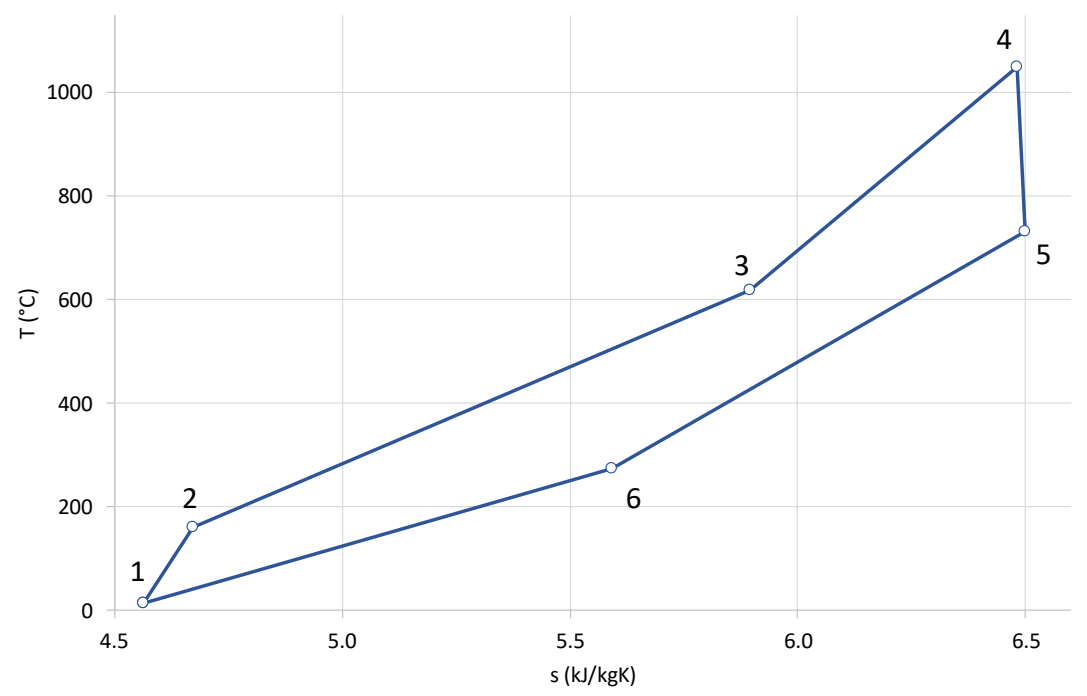

Figura 25.- Diagrama Temperatura-entropía ciclo Brayton regenerativo

Una vez conocidas las propiedades de cada estado se puede calcular el calor disponible:

$$
\dot{Q}_{\text {disp }}=\dot{m}_{g c} \bar{C} p_{g c}\left(T_{5}-T_{r e q}\right)
$$

Como se desconoce $\pi$ y $\mathrm{T}_{4}$ se tiene que iterar hasta obtener la $\mathrm{T}_{5}$ que proporciona el fabricante y el calor disponible reportado en la literatura para cada una de las MTG's y así conocer con que $\pi$ y $\mathrm{T}_{4}$ opera cada una de ellas.

Conocido lo anterior, se puede calcular el coeficiente de derrateo para cada una de las MTG's. Para ello se necesitan calcular los flujos de combustible, aire y gases de combustión reales para cada turbina en las distintas condiciones atmosféricas con el siguiente procedimiento:

1. Se calcula el calor suministrado que se requiere para calentar el aire:

$$
\dot{Q}_{\text {aire }}=\dot{m}_{\text {aire }} \bar{C} p_{\text {aire }}\left(T_{4}-T_{2}\right)
$$

2. Se calcula el flujo de combustible que se requiere para calentar el aire:

$$
\dot{m}_{\text {comba }}=\frac{\dot{Q}_{\text {aire }}}{P C I}
$$

3. Se calcula el calor que requiere el combustible para calentar el mismo combustible, tomando en cuenta el gradiente de temperatura desde la ambiental:

$$
\dot{Q}_{c}=\dot{m}_{\text {comba }} \bar{C} p_{\text {comb }}\left(T_{4}-T_{1}\right)
$$

4. Se calcula el flujo de combustible que se requiere para calentar el mismo combustible:

$$
\dot{m}_{\text {comb }}=\frac{\dot{Q}_{c}}{P C I}
$$

5. Se calcula el flujo total de combustible que se requiere tanto para calentar el aire como el combustible. 


$$
\dot{m}_{\text {combtotal }}=\dot{m}_{\text {comb }}+\dot{m}_{\text {comba }}
$$

6. Se calcula el flujo de gases de combustión:

$$
\dot{m}_{g c}=\dot{m}_{\text {combtotal }}+\dot{m}_{\text {aire }}
$$

Con los datos anteriores se calcula la potencia real de cada turbina:

$$
\dot{W}_{\text {real }}=\dot{m}_{g c} \bar{C} p_{g c}\left(T_{4}-T_{5}\right)
$$

Con esta nueva potencia se compara con la potencia ISO del fabricante y se obtiene una relación de potencias: coeficiente de derrateo a diferentes condiciones atmosféricas [47]. Además de obtenerse los trabajos y calores a las nuevas condiciones como se muestra en la Tabla 6.

Tabla 6.- Cálculos a las nuevas condiciones atmosféricas.

\begin{tabular}{lc}
\hline \multicolumn{1}{c}{ Componente } & Ecuación \\
\hline Trabajo de compresor & $\dot{W}_{c}=\dot{m}_{\text {aire }}\left(h_{2}-h_{1}\right)$ \\
\hline Trabajo de la turbina & $\dot{W}_{t}=\dot{m}_{g c}\left(h_{4}-h_{5}\right)$ \\
\hline Trabajo motor & $\dot{W}_{m}=\dot{W}_{t}-\dot{W}_{c}$ \\
\hline Calor suministrado & $\dot{Q}_{\text {sum }}=\bar{C} p_{\text {aire }}\left(T_{4}-T_{3}\right)$ \\
\hline Calor recuperado & $\dot{Q}_{\text {rec }}=\bar{C} p_{\text {aire }}\left(T_{3}-T_{2}\right)$ \\
\hline Flujo de combustible ahorrado & $\dot{m}_{\text {cahorro }}=\frac{\bar{C}_{\text {aire }} p_{\text {aire }}\left(T_{3}-T_{2}\right)}{P C I}$ \\
\hline Eficiencia & $\eta=\frac{\dot{W}_{m}}{\dot{Q}_{\text {sum }}}$ \\
\hline
\end{tabular}

\subsubsection{Hidrógeno}

El hidrógeno es el elemento más abundante, constituyendo casi tres cuartas partes de la masa del universo. Se encuentra en el agua, que cubre el $70 \%$ de la superficie terrestre, y en toda la materia orgánica. El hidrógeno es el elemento más simple del universo: se compone de un protón y un electrón. Tiene un PCI muy grande (120,011 kJ/kg) en comparación con el GN y GLP.

Para hacer uso de este combustible en una MTG se tiene que tomar en cuenta la cantidad de vapor de agua que estará presente al llevarse a cabo la reacción de hidrógeno con aire en la cámara de combustión. Al tener un elevado PCI la cantidad de combustible requerido para obtener la potencia nominal de las MTG's será menor al empleado usado con GN y GLP, y el flujo de gases de combustión en principio también decrece.

Para conocer la proporción en la que este flujo decrece se necesita hacer una metodología iterativa, partiendo en primera instancia con el flujo de gases de combustión que proporciona el fabricante (empleando GN, se toma este como referencia). A continuación, se describe la metodología propuesta para conocer los flujos de combustible, aire y gases de combustión que se requieren empleando hidrógeno. 
Partiendo de la ecuación de combustión empleando hidrógeno como combustible queda de la siguiente manera:

$$
N_{H_{2}} H_{2}+(1+x)\left(\frac{1}{2}\right) N_{H_{2}}\left(O_{2}+3.76 N_{2}\right) \rightarrow N_{H_{2}} H_{2} O+x\left(\frac{1}{2}\right) N_{H_{2}} O_{2}+(1+x)\left(\frac{1}{2}\right) N_{H_{2}} 3.76 N_{2}
$$

La potencia de la MTG está dada por:

$$
\begin{aligned}
& \dot{W}_{t}=\dot{m}_{g c} \Delta h=\dot{m}_{g c}\left(h_{4}-h_{5}\right) \\
& \dot{m}_{g c}=\dot{m}_{\text {aire }}+\dot{m}_{\mathrm{H}_{2} \mathrm{O}}
\end{aligned}
$$

Considerando que solo se tiene aire $\left(\dot{m}_{\mathrm{H}_{2} \mathrm{O}}=0\right.$ ) se puede conocer la cantidad de calor requerida en el proceso de 3 a 4 con la siguiente ecuación:

$$
\dot{Q}_{3-4}=\dot{m}_{g c}\left(h_{4}-h_{3}\right)=\dot{m}_{H_{2}} P C I_{H_{2}}
$$

A partir de los datos del fabricante se conoce el flujo de gases de combustión en condiciones ISO ( $\mathrm{P}=1.013$ bar y $\mathrm{T}=15^{\circ} \mathrm{C}$ ), por lo tanto, de la ecuación (36) se desconoce el flujo de combustible, que despejando nos queda:

$$
\dot{m}_{H_{2}}=\frac{\dot{m}_{g c}\left(h_{4}-h_{3}\right)}{P C I_{H_{2}}}
$$

Con esta primera aproximación se realiza el balance en la ecuación (33) en función del exceso de aire (x) y así obtener los flujos de agua, aire y nitrógeno que se tienen con esta cantidad de combustible. Posteriormente se calcula la cantidad de exceso de aire presente en la reacción con la siguiente ecuación:

$$
x=\frac{\dot{m}_{g c}-\dot{m}_{\mathrm{H}_{2} \mathrm{O}}-\dot{m}_{\mathrm{O}_{2}}-\dot{m}_{N_{2}}}{\dot{m}_{\mathrm{O}_{2}}+\dot{m}_{N_{2}}}
$$

Conocido el exceso de aire presente en esta primera aproximación se realiza el nuevo balance en la ecuación (33) con el valor obtenido de la ecuación (38). Con esto se calcula el porcentaje de vapor de agua (39) y aire presente en la reacción.

$$
x_{\mathrm{H}_{2} \mathrm{O}}=\frac{\dot{m}_{\mathrm{H}_{2} \mathrm{O}}}{\dot{m}_{g c}}
$$

Como existe un porcentaje de vapor de agua en la reacción, la potencia de la turbina (34) se puede re-escribir en función de esta fracción de la siguiente manera:

$$
\dot{W}_{t}=\dot{m}_{g c} x_{\mathrm{H}_{2} \mathrm{O}}\left(h_{4}-h_{5}\right)_{\mathrm{H}_{2} \mathrm{O}}+\dot{m}_{g c} x_{\text {aire }}\left(h_{4}-h_{5}\right)_{\text {aire }}
$$

La potencia obtenida en primera instancia no será la nominal, ya que partimos de que el flujo de gases de combustión era 100\% aire. Sin embargo, ahora se conoce la fracción de $\mathrm{H}_{2} \mathrm{O}$ presente en la reacción. Para conocer la proporción en la que disminuyen los flujos con respecto a los nominales 
se hace la relación de entalpias de vapor y aire (ecuación 41) en la cámara de combustión correspondiente al estado 4 del diagrama esquemático (Figura 15):

$$
R_{h}=\frac{h_{4_{H_{2} \mathrm{O}}}}{h_{4_{\text {aire }}}}
$$

Conocida la relación de entalpias $\left(\mathrm{R}_{\mathrm{h}}\right)$ se calcula el exceso de flujo de gases de combustión presente:

$$
\dot{m}_{g c_{\text {excess }}}=\frac{x_{\mathrm{H}_{2} \mathrm{O}}}{R_{h}}
$$

Por último, se resta el flujo que se tiene de exceso al flujo de gases de combustión nominal, y se obtiene un nuevo flujo de gases de combustión con el cual se recalcula la potencia de la turbina con la ecuación (40). En caso de no obtener la potencia nominal de la turbina, se requiere de hacer iteraciones hasta obtener esta. Es decir, se repite el proceso desde la ecuación (37) a (42).

Conocidos los nuevos flujos a condiciones ISO, se realizan los cálculos para el coeficiente de derrateo, siguiendo el mismo principio de la metodología planteada anteriormente cuando se utiliza GN y GLP, tomando en cuenta ahora la proporción de vapor de agua presente en los gases de combustión, así las ecuaciones de la Tabla 6 quedan como se muestran en la Tabla 7.

\begin{tabular}{|c|c|}
\hline Componente & Ecuación \\
\hline Trabajo de compresor & $\dot{W}_{c}=\dot{m}_{\text {aire }}\left(h_{2}-h_{1}\right)$ \\
\hline Trabajo de la turbina & $\dot{W}_{t}=\dot{m}_{g c} x_{\mathrm{H}_{2} \mathrm{O}}\left(h_{4}-h_{5}\right)_{\mathrm{H}_{2} \mathrm{O}}+\dot{m}_{g c} x_{\text {aire }}\left(h_{4}-h_{5}\right)_{\text {aire }}$ \\
\hline Trabajo motor & $\dot{W}_{m}=\dot{W}_{t}-\dot{W}_{c}$ \\
\hline Calor suministrado & $\dot{Q}_{\text {sum }}=\left(\dot{m}_{g c} x_{\mathrm{H}_{2} \mathrm{O}} h_{4_{\mathrm{H}_{2} \mathrm{O}}}+\dot{m}_{g c} x_{\text {aire }} h_{4_{\text {aire }}}\right)-\dot{m}_{\text {aire }} h_{3}$ \\
\hline Calor recuperado & $\dot{Q}_{\text {rec }}=\dot{m}_{\text {aire }} h_{1}-\left(\dot{m}_{g c} x_{H_{2} \mathrm{O}} h_{6_{H_{2} \mathrm{O}}}+\dot{m}_{g c} x_{\text {aire }} h_{6_{\text {aire }}}\right)$ \\
\hline Flujo de combustible ahorrado & $\dot{m}_{\text {cahorro }}=\frac{\dot{m}_{\text {aire }} \bar{C} p_{\text {aire }}\left(T_{3}-T_{2}\right)}{P C I}$ \\
\hline Eficiencia & $\eta=\frac{\dot{W}_{m}}{\dot{Q}_{\text {sum }}}$ \\
\hline
\end{tabular}

Tabla 7.- Cálculos a las nuevas condiciones atmosféricas usando $\mathrm{H}_{2}$

\subsection{Análisis térmico de un generador de vapor convencional}

Para este estudio se seleccionaron los GV's de 4 pasos Dry-Back y se eligió una gama de GV Cleaver Brooks como se muestra en la Tabla 8 [48], donde los parámetros mostrados son los fundamentales de conocer para poder hacer los cálculos correspondientes. 


\section{Arreglo y distribución de haz de tubos}

Existen 3 tipos de arreglos de tubos, como se muestran en la Figura 26, donde los haz de tubos del inciso a) son de forma matricial lineal y los haz de tubos de los incisos b) y c) son de forma matricial escalonada [49]. Normalmente los GV's pirotubulares trabajan con un arreglo matricial escalonado triangular (inciso b). Para este tipo de arreglos es necesario conocer o proponer la distancia que deben tener entre cada uno de ellos de centro a centro de forma longitudinal (X1) y transversal $(\mathrm{Xd})$, las cuales se calculan con las siguientes ecuaciones:

$$
\begin{aligned}
& X l=1.5 d+d \\
& X t=2.5 d+d \\
& X d=\sqrt{X l^{2}+\left(\frac{X t}{2}\right)^{2}}
\end{aligned}
$$

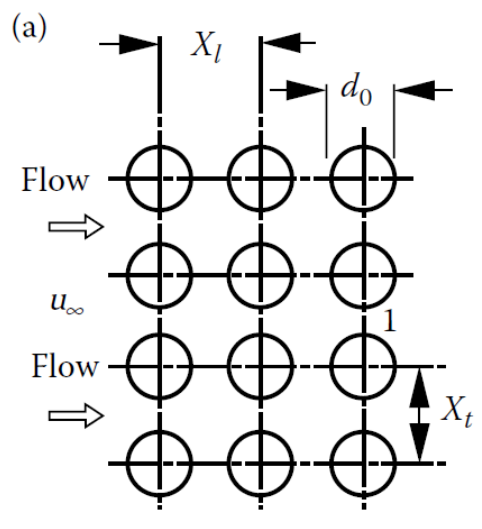

(b)

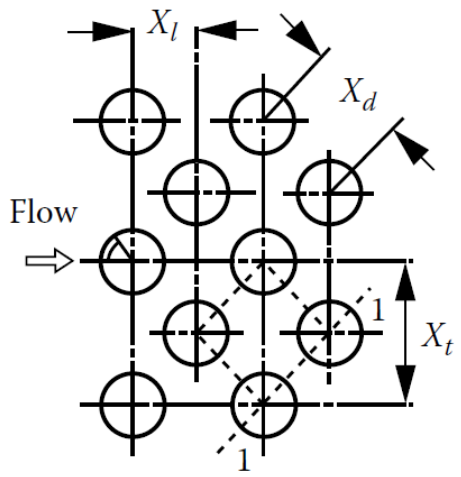

(c)

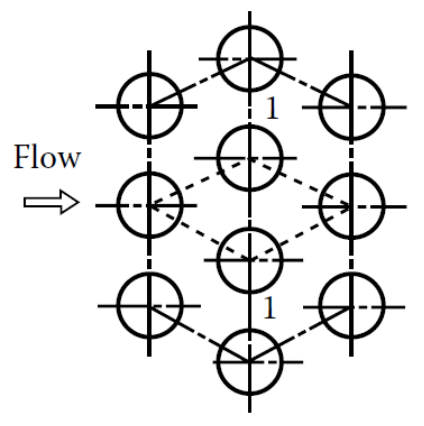

Figura 26.- Tipos de arreglo de tubos

Para calcular el número de tubos del GV se necesita conocer el área de transferencia de calor, la cual nos proporciona el fabricante, además como se conocen las dimensiones de los tubos se puede calcular el área de cada uno de ellos y finalmente obtener el número de tubos de la siguiente manera:

$$
N o_{t u}=\frac{A_{\text {fireside }}}{A_{t u}}=\frac{A_{\text {fireside }}}{\pi D_{\text {ext }} L}
$$

Conocido el número de tubos y la distancia que debe haber entre cada uno de ellos se puede establecer la distribución de los tubos en el GV, tomando en cuenta que el área donde no hay tubos es de aproximadamente 1/4D coraza. En las Figura 27 y 28 se muestra el arreglo de tubos a modo de ejemplo para el GV de 40 BHP con una distribución matricial triangular. En las Figura 29 y 30 se muestra la carcasa del GV con las entradas de flujo de agua y gases de combustión, también se muestran las mamparas que dirigen el flujo de los 4 pasos que consta este $\mathrm{GV}$. 


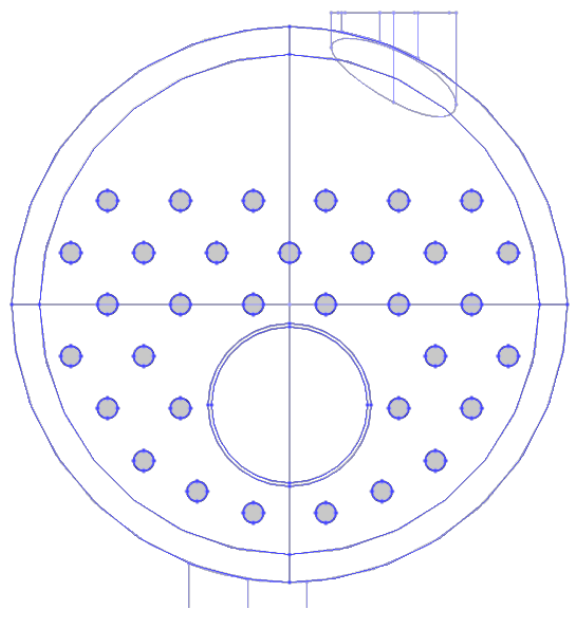

Figura 27.- Arreglo de tubos

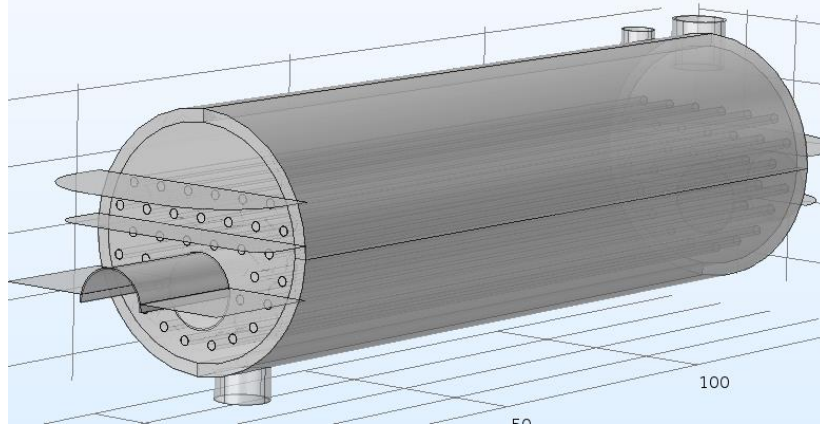

Figura 29.- Vista de los pasos de flujo

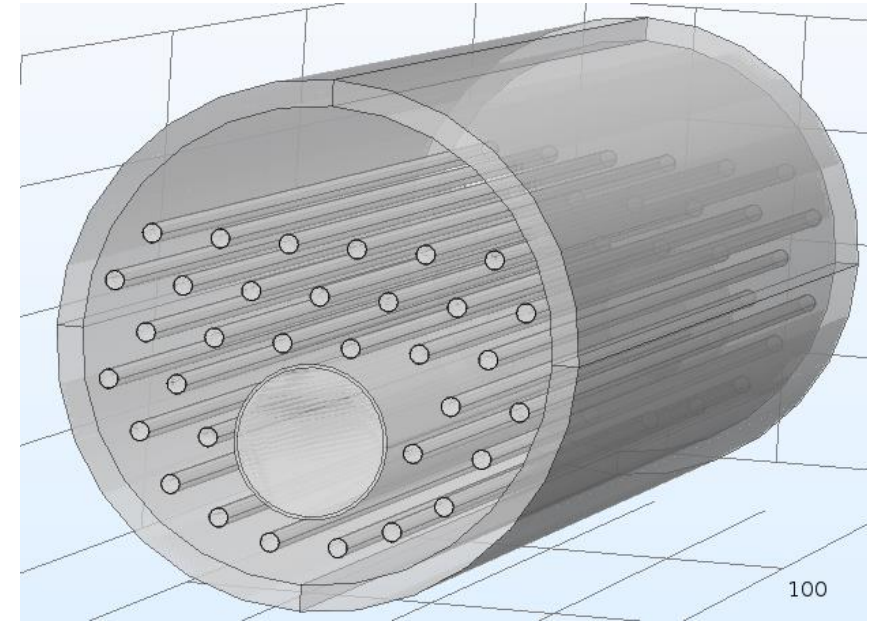

Figura 28.- Arreglo de tubos vista $3 D$

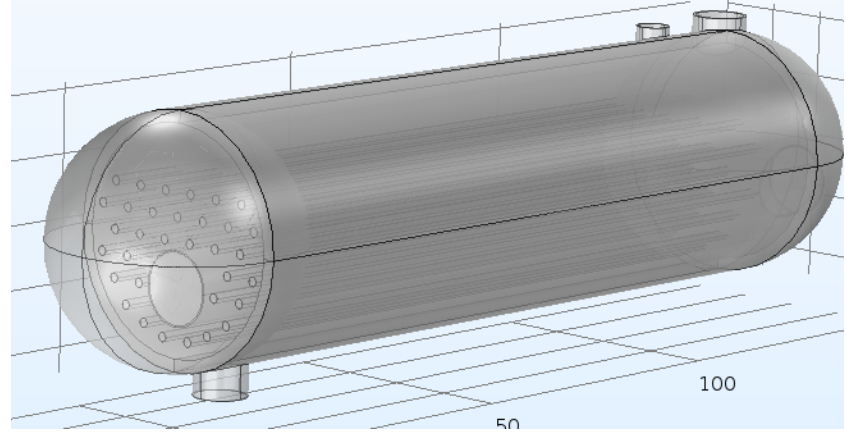

Figura 30.- Vista final del GV en $3 D$

En la Figura 31 se muestra el diagrama esquemático de un GV. Estos GV's cuentan con dos alimentaciones de aire: primario y secundario. Se pretende sustituir el flujo de aire secundario por los gases provenientes de una MTG. El objetivo es aprovechar el calor de esos gases para reducir el consumo de combustible, derivado que viene con un elevado exceso de aire no perturbaría el sistema de combustión convencional en el GV. Bajo esta premisa se debería reducir el consumo de combustible. Sin embargo, cabe hacer la pregunta si la reducción en el consumo de combustible hace rentable la inversión del acoplamiento de estos gases. También se deberá por lo tanto conocer la magnitud de la perturbación en los coeficientes de transferencia de calor. Para ello primero se necesita conocer el flujo de aire que requieren los GV's de la Tabla 8 y saber si con las MTG bajo estudio se pueden cubrir esas demandas de aire y sustituirlo, de tal forma que se evite una cantidad determinada de combustible. Para ello se desarrolló la siguiente metodología [50] [51]:

Tabla 8.- Especificaciones de GV's Cleaver Brooks de 4 paso Dry-Back de 15 a 100 BHP

\begin{tabular}{|l|c|c|c|c|c|c|c|c|c|}
\hline BHP & $\mathbf{1 5}$ CB & $\mathbf{2 0 ~ C B}$ & $\mathbf{3 0}$ CB & $\mathbf{4 0}$ CB & $\mathbf{5 0}$ CB & $\mathbf{6 0}$ CB & $\mathbf{7 0 ~ C B}$ & $\mathbf{8 0}$ CB & $\mathbf{1 0 0}$ CB \\
\hline $\begin{array}{l}\text { Producción de vapor [kg/s] } \\
\text { desde Y hasta 100 }{ }^{\circ} \mathrm{C}\end{array}$ & 0.065 & 0.086 & 0.130 & 0.173 & 0.217 & 0.260 & 0.304 & 0.347 & 0.434 \\
\hline Potencia del GV [kW] & 147.12 & 196.36 & 294.24 & 392.42 & 490.60 & 588.78 & 686.67 & 784.84 & 981.20 \\
\hline $\begin{array}{l}\text { Superficie de calentamiento } \\
\text { (Fireside) [m }{ }^{2} \text { ] }\end{array}$ & 6.97 & 9.29 & 13.94 & 18.58 & 23.23 & 27.87 & 32.52 & 37.16 & 46.45 \\
\hline Flujo de combustible [kg/s] & 0.0033 & 0.0044 & 0.0067 & 0.0088 & 0.0111 & 0.0133 & 0.015 & 0.017 & 0.022 \\
\hline Diámetro del tubo [m] & 0.04109 & 0.04229 & 0.04037 & 0.04011 & 0.05867 & 0.05867 & 0.05867 & 0.05867 & 0.05867 \\
\hline Espesor del tubo [m] & & & & & 0.00483 & & & \\
\hline Longitud de los tubos [m] & 2.16 & 2.16 & 2.62 & 3.28 & 2.97 & 2.97 & 3.96 & 3.96 & 4.45 \\
\hline
\end{tabular}




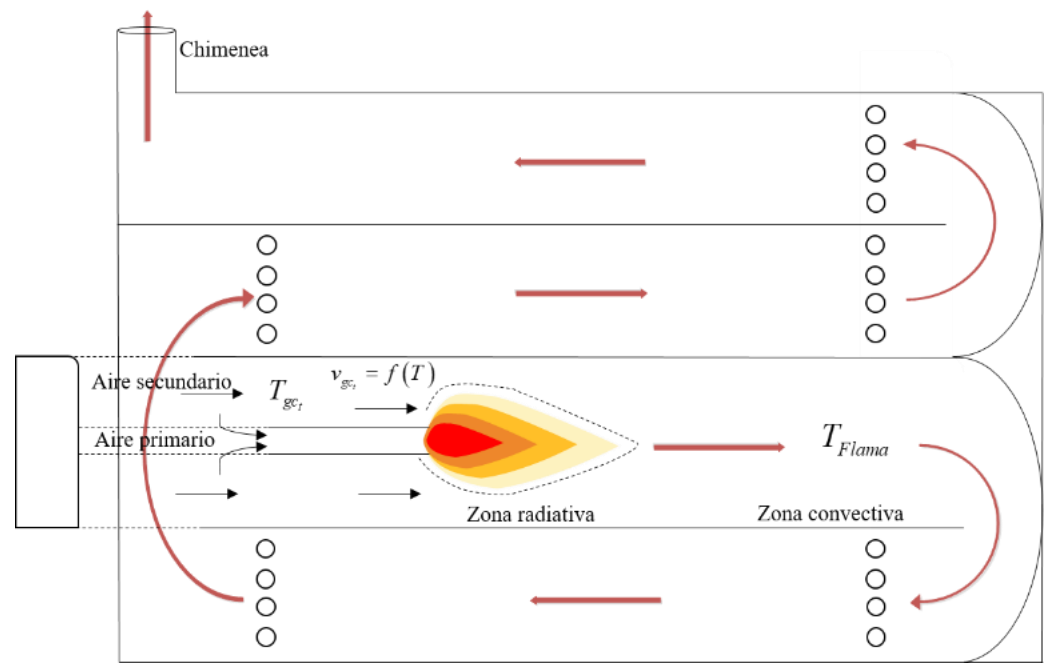

Figura 31.- Diagrama esquemático de un GV de 4 pasos Dry-Back

1. A partir de los datos del fabricante se obtiene la cantidad de vapor estándar disponible mediante:

$$
\dot{Q}_{v a p}=\dot{m}_{v a p N o m} \Delta h
$$

Donde ese $\Delta h$ está en función de las entalpias de condiciones de operación y ambientales, por lo tanto, al ser definidas para operar bajo nuevas condiciones ( $\mathrm{P}=10$ bar saturado) se recalcula el flujo de vapor real con la siguiente ecuación:

$$
\dot{m}_{\text {vap Re } a l}=\frac{\dot{m}_{\text {vapNom }}}{f e}
$$

Donde: fe es el factor de evaporación.

2. Conocido el flujo de vapor real, se puede calcular el calor suministrado por el combustible:

$$
\dot{Q}_{\text {sum }}=\frac{\dot{Q}_{\text {vap }}}{\eta}
$$

3. Se calcula el flujo de combustible:

$$
\dot{m}_{\text {comb }}=\frac{\dot{Q}_{\text {sum }}}{P C I}
$$

4. Posteriormente el flujo de gases de combustión en función del exceso de aire, el cual varia de $0-50 \%$ :

$$
\dot{m}_{g c}=\left(R A C_{\text {real }}+1\right) \dot{m}_{\text {comb }}
$$

5. Se obtiene el flujo de aire del GV de forma estequiométrica $(x=0)$ y con exceso de aire $(\mathrm{x} \neq 0)$.

$$
\dot{m}_{\text {aire }}=\dot{m}_{g c}-\dot{m}_{\text {comb }}
$$


Conocidos los flujos que requieren los GV's, se puede hacer una primera evaluación de estos con las MTG C30 y C65, y conocer la gama de GV's que puede cubrir cada una de ellas.

\subsubsection{Cálculo del coeficiente convectivo del lado de los gases h1}

Conocidos los flujos másicos en función del exceso de aire, se puede calcular el coeficiente convectivo del lado de los gases (haz de tubos) [21] con la siguiente metodología:

1. Se obtiene el flujo de gases de combustión para cada tubo:

$$
\dot{m}_{g c, t u}=\frac{\dot{m}_{g c}}{N_{T}}
$$

2. Se determina la velocidad de los gases por tubo:

$$
u_{g c, t u}=\frac{\dot{V}_{g c, t u}}{\rho_{g c\left(\bar{T}_{g c}\right)} A_{t u}}
$$

Donde la densidad de los gases de combustión está en función de una temperatura promedio que llevan éstos según:

$$
\bar{T}_{g c}=\frac{T_{\text {Flama }}-T_{p r o p, g c}}{2}
$$

3. Conocida la velocidad de los gases de combustión se determina el Reynolds:

$$
\operatorname{Re}=\frac{u_{t u} \rho D_{\text {int }}}{\mu}
$$

4. A partir del número de Reynolds se emplean correlaciones del número de Nusselt dependiendo del régimen de flujo obtenido (laminar, transitorio o turbulento). Con el número de Nusselt se obtiene el coeficiente global interno convectivo (del lado de los gases).

$$
h_{1}=\frac{N u k}{D_{\mathrm{int}}}
$$

Donde el Nusselt se determina en función del régimen de flujo [21]:

Flujo turbulento: $N u=0.22 \operatorname{Re}^{0.8} \operatorname{Pr}^{0.5}$

Flujo en transición: $N u=\frac{(f / 2)(\operatorname{Re}-1000) \operatorname{Pr}}{1+12.7 \sqrt{f / 2}\left(\operatorname{Pr}^{2 / 3}-1\right)}$

Donde: $f=(1.58 \ln \operatorname{Re}-3.28)^{-2}$ 


\subsubsection{Cálculo del coeficiente convectivo del lado del agua $\mathbf{h}_{2}$}

Para el cálculo del coeficiente convectivo del lado de la coraza se debe tomar en cuenta que hay un cambio de fase. En la Figura 32 se muestra la curva típica de la ebullición del agua. Se observa que la parte de ebullición nucleada (nucleate boiling) es donde ocurre el cambio de fase [52]. La ebullición puede ser tratada como una forma de transferencia de calor por convección debido a la formación de corrientes convectivas, producto de la formación de burbujas ascendentes que ocasionan un movimiento al fluido de trabajo.

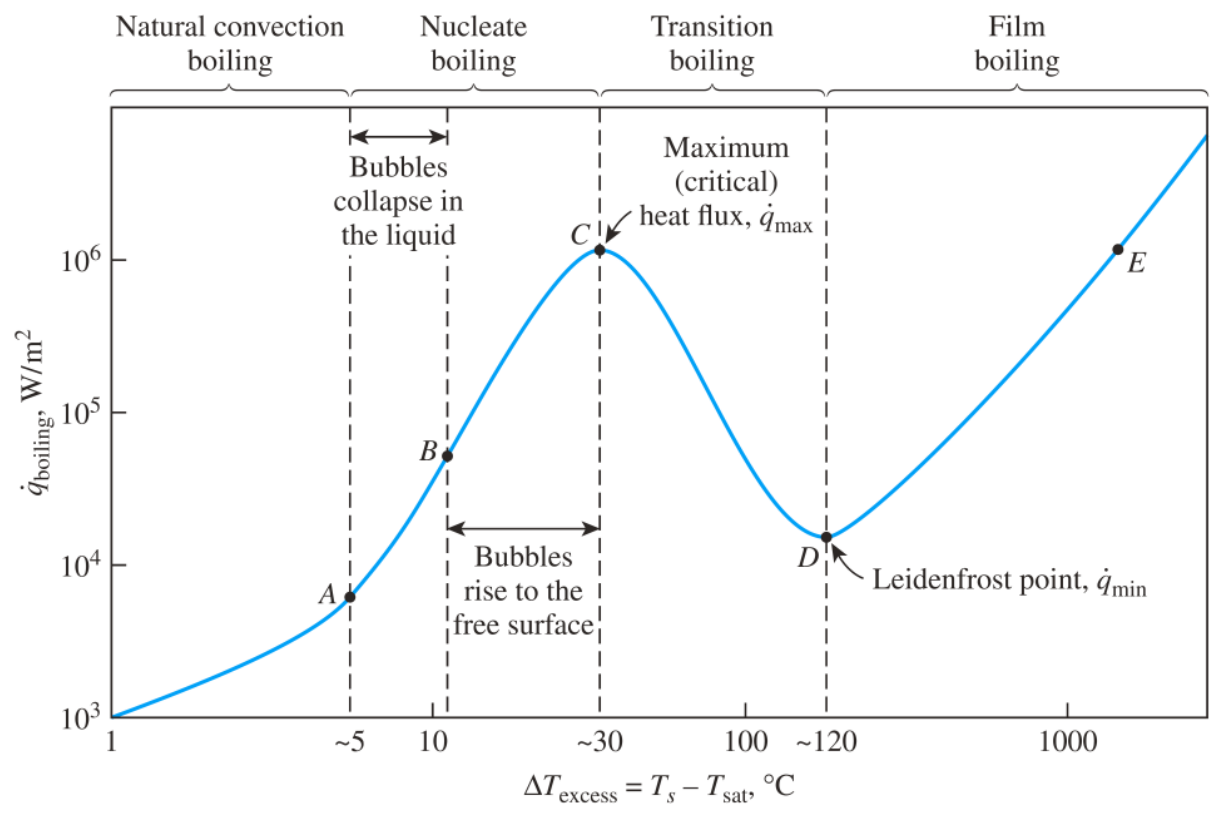

Figura 32.- Curva típica de ebullición del agua a 1 atm.

En la literatura existen distintas correlaciones en las que se toman en cuentan la zona nucleada. La utilizada en este trabajo es la propuesta por Gorenflo [53], quien propone un método para el cálculo del coeficiente de transferencia de calor en la zona de ebullición nucleada, el cual está ligado a la presión reducida del fluido de trabajo.

La presión reducida se define como la razón entre la presión real y la presión critica del agua, y está involucrada en las correlaciones para el análisis del fenómeno de ebullición junto con el efecto de la rugosidad del material.

$$
P_{r}=\frac{P_{s a t}}{P_{c r i}}
$$

Su método utiliza un coeficiente de transferencia de calor de referencia $\alpha_{0}$ especificado para cada fluido en las siguientes condiciones de referencia fijas de $\mathrm{p}_{\mathrm{r} 0}=0, \mathrm{R}_{\mathrm{p} 0}=0.4 \mu \mathrm{m}$ y q $\mathrm{q}_{0}=20,000$ $\mathrm{W} / \mathrm{m}^{2}$. Sus valores de $\alpha_{0}$ se muestra en la Tabla 33 del Apéndice C. El coeficiente de transferencia de calor de ebullición nucleado $\mathrm{h}_{2}$ (del lado del agua) en otras condiciones de presión, flujo de calor $\mathrm{y}$ rugosidad se calcula luego en relación con el coeficiente de transferencia de calor de referencia utilizando la siguiente expresión: 


$$
h_{2}=\alpha_{0} F_{P F}\left(\frac{q}{q_{0}}\right)^{n f}\left(\frac{R_{p}}{R_{p_{0}}}\right)^{0.133}
$$

Donde $F_{P F}$ es el factor de corrección de presión:

$$
F_{P F}=1.2 P_{r}^{0.27}+2.5 P_{r}+\frac{P_{r}}{1-P_{r}}
$$

El efecto de la presión reducida en su exponente $n f$ para el término de flujo de calor está dado por:

$$
n f=0.9-0.3 P_{r}^{0.3}
$$

$\mathrm{Su}$ valor disminuye al aumentar la presión reducida, lo cual es típico de los datos experimentales. La rugosidad de la superficie es $R p$ en $\mu \mathrm{m}$ y se establece en $0.4 \mu \mathrm{m}$ cuando se desconoce. El método anterior es para todos los fluidos excepto el agua y el helio; para el agua las ecuaciones correspondientes son:

$$
\begin{aligned}
& F_{P F}=1.73 P_{r}^{0.27}+\left(6.1+\frac{0.68}{1-P_{r}}\right) P_{r}^{2} \\
& n f=0.9-0.3 P_{r}^{0.15}
\end{aligned}
$$

Este método es aplicable en el intervalo de presión reducida de 0.0005 a 0.95 . Para fluidos no listados en el Apéndice C, se pueden ingresar valores experimentales en las condiciones de referencia, o emplear otra correlación para estimar $\alpha_{0}$. Para los fluidos en la lista, este método proporciona resultados precisos en un intervalo muy amplio de flujo de calor y presión, y es probablemente el más confiable [53].

\subsubsection{Cálculo del coeficiente global de transferencia de calor $\mathbf{U}$}

La tasa de transferencia de calor total será por: convección-conducción-convección, como se muestra en la Figura 33. Conocidos los coeficientes convectivos del lado de los gases $\left(\mathrm{h}_{1}\right)$ y del agua $\left(\mathrm{h}_{2}\right)$, solo hace falta calcular la parte de transferencia de calor por conducción en el haz de tubos y posteriormente poder calcular el coeficiente global, para ello se lleva a cabo el siguiente procedimiento:

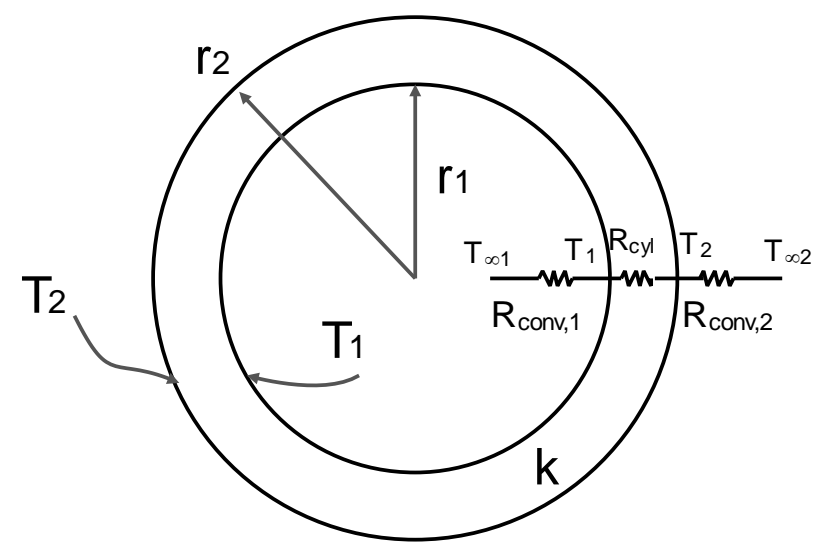

Figura 33.- Resistencias térmicas en coordenadas cilíndricas. 
Para la transferencia de calor por conducción se parte de la ecuación de Fourier, que es la transferencia de calor en una pared:

$$
\dot{Q}_{\text {cond }, c y l}=-k A \frac{d T}{d r}
$$

Donde: k es la conductividad térmica del material

A es el área de transferencia de calor

Resolviendo la ecuación por separación de variables e integrando queda:

$$
\int_{r=r_{1}}^{r_{2}} \frac{\dot{Q}_{\text {cond }, c y l}}{A} d r=-\int_{T=T_{1}}^{T_{2}} k d T
$$

Donde: $A=2 \pi r L$

$$
\dot{Q}_{\text {cond }, c y l}=2 \pi L k \frac{T_{1}-T_{2}}{\ln \left(r_{2} / r_{1}\right)}
$$

La ecuación anterior se puede reescribir en función de las resistencias térmicas de conducción del material:

$$
\dot{Q}_{\text {cond }, c y l}=\frac{T_{1}-T_{2}}{R_{c y l}}
$$

Donde: $R_{c y l}=\frac{\ln \left(r_{2} / r_{1}\right)}{2 \pi L k}$

Para la transferencia de calor por convección se parte de la ecuación de Newton de la ley de enfriamiento:

$$
\dot{Q}_{\text {conv }, c y l}=h A_{s}\left(T_{s}-T_{\infty}\right)
$$

Reescribiendo la ecuación anterior en términos de resistencia térmica por convección queda:

$$
\dot{Q}_{\text {conv }, c y l}=\frac{\left(T_{s}-T_{\infty}\right)}{R_{\text {conv }}}
$$

Donde: $R_{c o n v}=\frac{1}{h A_{s}}$

Por lo tanto, la ecuación de la tasa de transferencia de calor total queda de la siguiente manera:

$$
\dot{Q}_{\text {total }}=\frac{\left(T_{\infty_{1}}-T_{\infty_{2}}\right)}{R_{\text {total }}}
$$

Donde: $R_{\text {total }}=\frac{1}{h_{1} A_{1}}+\frac{\ln \left(\frac{r_{2}}{r_{1}}\right)}{2 \pi k L}+\frac{1}{h_{2} A_{2}}$ 
Esta última ecuación conviene ponerla en términos de la ley de enfriamiento de Newton para obtener el coeficiente de transferencia de calor global.

$$
\dot{Q}=U A_{0} \Delta T_{\text {ln }}
$$

Donde el coeficiente global de transferencia de calor $U$ también se define en términos de resistencias:

$$
U A_{0}=\frac{1}{R_{\text {total }}}
$$

Donde: $A_{0}=2 \pi r_{\text {prom }} L_{t u}$

$$
r_{\text {prom }}=\frac{r_{1}+\left(r_{2}-r_{1}\right)}{2}
$$

Sustituyendo $\mathrm{R}_{\text {total }}$ en la ecuación (74) queda:

$$
U A_{0}=\frac{1}{\frac{1}{h_{1} A_{1}}+\frac{\ln \left(r_{2} / r_{1}\right)}{2 \pi k L}+\frac{1}{h_{2} A_{2}}}
$$

Con esta última ecuación se realizan los cálculos para obtener el coeficiente global de transferencia de calor en función del exceso de aire en cada GV con y sin gases de combustión provenientes de una MTG. Sin embargo, como las condiciones de vapor son fijas: a una presión de 10 bar y saturado; el coeficiente convectivo $\mathrm{h}_{2}$ se considera en primera instancia constante. Además, al ser este mucho mayor en magnitud con respecto a $h_{1}$, el coeficiente que aporta la mayor resistencia es $\mathrm{h}_{1}$ y por lo tanto U tendrá un valor muy cercano éste.

\subsubsection{Determinación de la caída de presión en el haz de tubos}

Se sabe que siempre habrá caídas de presión a lo largo del haz de tubos, debido principalmente por la fricción. Para que se tenga una menor caída de presión en un GV el arreglo de tubos en cada paso es importante, ya que se debe reducir el área de la sección transversal para cada paso sucesivo de gas, pues al enfriarse el gas su volumen disminuye. Para ello se van reduciendo el número de tubos en cada paso como se muestra en la Figura 34 [54].

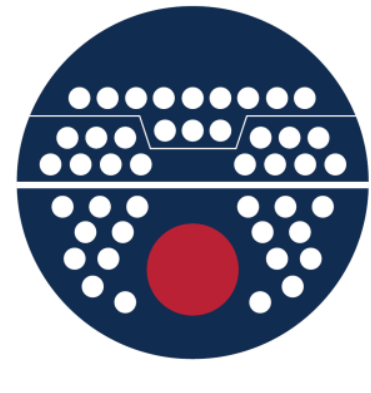

1er paso
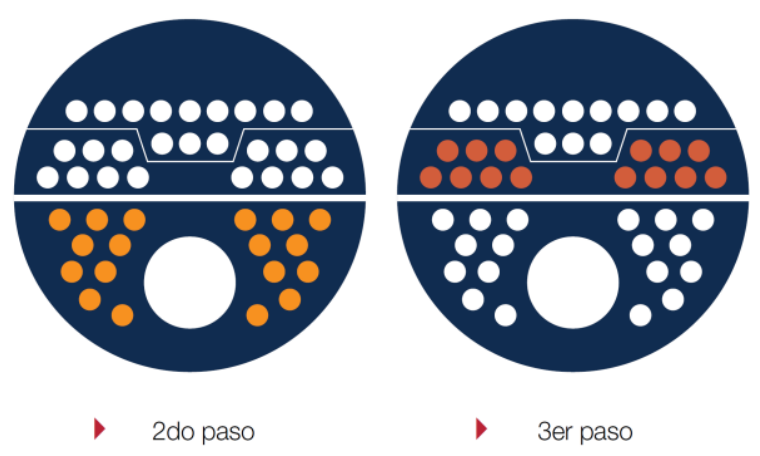

- 3er paso

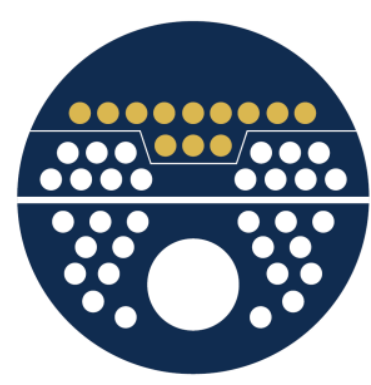

4to paso

Figura 34.- Ejemplo de arreglo de tubos de 4 pasos.

La caída de presión en el haz de tubos se calcula de la siguiente manera [49]: 


$$
\Delta p=4 f \frac{L N_{t u}}{d_{\text {int }}} \frac{G^{2}}{2 \rho}
$$

Donde:

G: es la velocidad de la masa $G=u_{m} \rho$

f: es el coeficiente de fricción, el cual está en función del régimen del Reynolds como se muestra en la Tabla 9.

Tabla 9.- Correlaciones del factor de fricción [49].

\begin{tabular}{|l|c|c|}
\hline \multicolumn{1}{|c|}{ Autor } & \multicolumn{1}{c|}{ Correlación } & Restricciones \\
\hline Blasius & $f=\frac{\tau_{w}}{\rho u_{m}^{2} / 2}=0.0791 \mathrm{Re}^{-0.25}$ & $4 x 10^{3}<\operatorname{Re}<10^{5}$ \\
\hline $\begin{array}{l}\text { Drew, Koo y McAdams Karman- } \\
\text { Nikuradse }\end{array}$ & $f=0.00140+0.125 \mathrm{Re}^{-0.32}$ & $4 x 10^{3}<\operatorname{Re}<5 \times 10^{6}$ \\
\hline Filonenko & $f=\left(3.64 \log _{10} \operatorname{Re}-3.28\right)^{-2}$ & $2 x 10^{3}<\operatorname{Re}<6 \times 10^{3}$ \\
\hline
\end{tabular}

Conocida la caída de presión se puede calcular la potencia del soplador para que los gases logren salir del GV. Este soplador debe vencer la caída de presión existente y se calcula con:

$$
\dot{W}_{p}=\frac{1}{\eta_{p}} \frac{\dot{m}}{\rho} \Delta p
$$

Donde el termino $\frac{\dot{m}}{\rho} \Delta p$ representa la potencia isoentrópica mínima requerida y $\eta_{p}$ es la eficiencia isoentrópica del soplador.

\subsection{Análisis térmico de un generador de vapor que opera con los gases de combustión de una MTG}

Los gases de combustión de una MTG salen a relativamente altas temperaturas, alrededor de los 270 a $320{ }^{\circ} \mathrm{C}$, los cuales en sistemas convencionales son mandados directamente a la atmósfera. Razón por la cual es importante realizar un análisis de factibilidad de la implementación de un sistema continuo de aprovechamiento de los gases de combustión de la MTG en un GV convencional que evitará la inversión de un recuperador de calor (Figura 35).
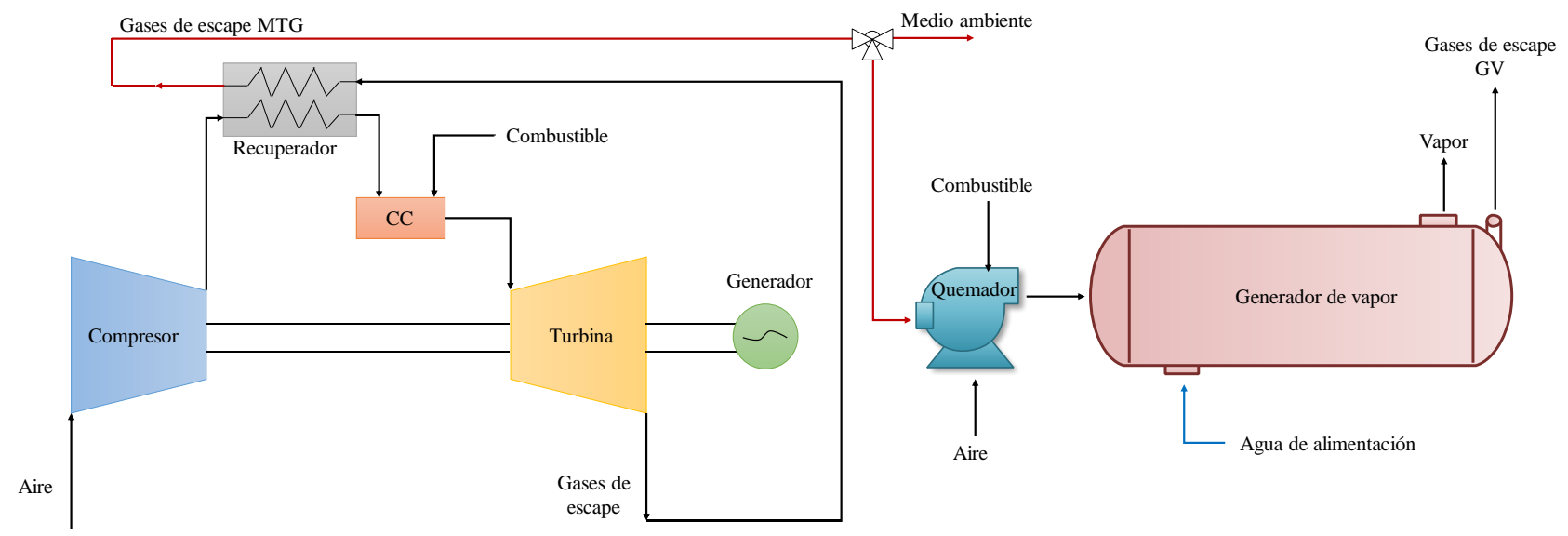

Figura 35.- Diagrama esquemático del acoplamiento de gases de combustión de una MTG a un GV. 
Para hacer el análisis del acoplamiento de los gases efluentes de una MTG a un GV, lo primero que se necesita conocer es su composición; se obtiene con la ec. (19) para cuando esta reacción se lleva a cabo con una cierta cantidad de moles $\left(N_{T}\right)$ de combustible y exceso de aire. Con los datos que proporciona el fabricante de las MTG se conoce la cantidad de combustible con la que se alimentan y se calcula el número de $N_{T}$ alimentados. Con la ecuación (21) se conoce la MM del combustible y se determina $N_{T}$ :

$$
N_{T}=\frac{\dot{m}_{c o m b T}}{M M_{c}}
$$

La ecuación (5) se puede reescribir para determinar la composición de los gases de escape y queda:

$$
N_{T} C_{n} H_{m}+\left(1+x_{T}\right) N_{T}\left(n+\frac{m}{4}\right)\left(O_{2}+3.76 N_{2}\right) \rightarrow N_{T} n C O_{2}+N_{T} \frac{m}{2} H_{2} O+x_{T} N_{T}\left(n+\frac{m}{4}\right) O_{2}+\left(1+x_{T}\right) N_{T}\left(n+\frac{m}{4}\right) 3.76 N_{2}
$$

Conocida la composición de los gases de escape y el flujo de los mismos se puede calcular el calor disponible con la ec. (25) y considerando el valor de $\mathrm{T}_{\mathrm{amb}}=25^{\circ} \mathrm{C}$. Con la cantidad de calor por los gases de combustión y el calor requerido del GV usando la ec. (49), se calcula el nuevo flujo de combustible que requiere el GV al introducir los gases de combustión a la misma:

$$
\begin{aligned}
& \dot{Q}_{\text {comb } 2}=\dot{Q}_{\text {sum }}-\dot{Q}_{g c T} \\
& \dot{m}_{\text {comb } 2}=\frac{\dot{Q}_{\text {comb } 2}}{P C I}
\end{aligned}
$$

Con este flujo de combustible del GV se calcula la cantidad de oxigeno que requiere de forma estequiométrica:

$$
N_{G V} C_{n} H_{m}+N_{G V}\left(n+\frac{m}{4}\right)\left(O_{2}+3.76 N_{2}\right) \rightarrow N_{G V} n C O_{2}+N_{G V} \frac{m}{2} H_{2} O+N_{G V}\left(n+\frac{m}{4}\right) 3.76 N_{2}
$$

Empleando la composición de los gases provenientes de la MTG se realiza el balance de masa de la combustión, de suerte de conocer si existe exceso o deficiencia de oxigeno con esos gases:

$$
\begin{aligned}
& N_{G V} C_{n} H_{m}+N_{T} n C O_{2}+N_{T} \frac{m}{2} H_{2} O+x_{T} N_{T}\left(n+\frac{m}{4}\right) O_{2}+\left(1+x_{T}\right) N_{T}\left(n+\frac{m}{4}\right) 3.76 N_{2} \\
& \rightarrow N_{T} n C O_{2}+N_{G V} n C O_{2}+N_{T} \frac{m}{2} H_{2} O+N_{G V} \frac{m}{2} H_{2} O+\alpha O_{2}+\left(1+x_{T}\right) N_{T}\left(n+\frac{m}{4}\right) 3.76 N_{2}
\end{aligned}
$$

Donde $\alpha$ es la cantidad de oxigeno presente en los gases de escape del GV.

Si: $\quad \alpha<0$ se tiene déficit de oxigeno

$\alpha=0$ es estequiométrico

$\alpha>0$ se tiene exceso de oxigeno

Cuando se tiene $\alpha<0$, al tener un déficit de oxigeno se emplean los gases de combustión provenientes de la MTG en su totalidad, pero se requiere de inyectar una cierta cantidad de aire extra para poder alcanzar las condiciones de operación deseadas. Tomando en cuanto esto, la ecuación de combustión queda: 


$$
\begin{aligned}
& N_{G V} C_{n} H_{m}+N_{T} n \mathrm{CO}_{2}+N_{T} \frac{m}{2} \mathrm{H}_{2} \mathrm{O}+x_{T} N_{T}\left(n+\frac{m}{4}\right) O_{2}+\left(1+x_{T}\right) N_{T}\left(n+\frac{m}{4}\right) 3.76 N_{2}+\alpha\left(1+x_{G V}\right)\left(O_{2}+3.76 N_{2}\right) \\
& \rightarrow N_{T} n \mathrm{CO}_{2}+N_{G V} n C O_{2}+N_{T} \frac{m}{2} H_{2} O+N_{G V} \frac{m}{2} H_{2} O+x_{G V} \alpha O_{2}+\left(1+x_{T}\right) N_{T}\left(n+\frac{m}{4}\right) 3.76 N_{2}+\left(1+x_{G V}\right) \alpha 3.76 N_{2}
\end{aligned}
$$

Cuando se tiene un valor de $\alpha>0$, al tener un exceso de oxígeno, significa que se debe desviar una parte del flujo de gases de combustión provenientes de la MTG. Como los GV's normalmente operan a un $10 \%$ de exceso de aire, se buscaría desviar la menor cantidad de gases de combustión de tal forma que no se requiera ingresar nuevo aire como en el caso anterior y aprovechar el que contienen estos gases, para ello se realiza la siguiente metodología.

La ecuación de combustión en este caso queda:

$$
\begin{aligned}
& \left(N_{x}+N_{G V}\right) C_{n} H_{m}+\varepsilon\left\{N_{T}\left[n C O_{2}+\frac{m}{2} H_{2} O+x_{T}\left(n+\frac{m}{4}\right) O_{2}+\left(1+x_{T}\right)\left(n+\frac{m}{4}\right) 3.76 N_{2}\right]\right\} \rightarrow\left[\left(N_{x}+N_{G V}\right)+\varepsilon N_{T}\right] n C O_{2} \\
& +\left[\left(N_{x}+N_{G V}\right)+\varepsilon N_{T}\right] \frac{m}{2} H_{2} O+\varepsilon N_{T}\left(1+x_{T}\right)\left(n+\frac{m}{4}\right) 3.76 N_{2}+\omega O_{2}
\end{aligned}
$$

Donde:

$\varepsilon$ : es la cantidad de flujo de gases de combustión empleado, [kmol/s]

$\omega$ : la cantidad de exceso de oxígeno, $[\mathrm{kmol} / \mathrm{s}]$

$\mathrm{N}_{\mathrm{x}}$ : cantidad de moles de combustible extras a emplear, [kmol/s]

El termino $\mathrm{N}_{\mathrm{x}}$ aparece en la ec. (85) debido a la cantidad de energía que se desvía en los gases de combustión y esta debe ser compensada ingresando más combustible para llegar a las condiciones de operación deseadas.

A partir de los datos de operación de los GV's convencionales se puede hacer un estimado del $\% \mathrm{O}_{2}$ que equivale a un determinado exceso de aire. Que en este caso se fijará a un $10 \%$ de exceso de aire. Como ya se mencionó anteriormente los GV's normalmente operan a esta condición.

En la ec. (82) se mostró la ecuación de combustión de forma estequiométrica para los GV's convencionales, reescribiendo esta ecuación en función del exceso de aire queda:

$$
\begin{aligned}
& N_{G V} C_{n} H_{m}+N_{G V}\left(1+x_{G V}\right)\left(n+\frac{m}{4}\right)\left(O_{2}+3.76 N_{2}\right) \rightarrow N_{G V} n C O_{2}+N_{G V} \frac{m}{2} H_{2} O+x_{G V} N_{G V}\left(n+\frac{m}{4}\right) O_{2} \\
& +N_{G V}\left(1+x_{G V}\right)\left(n+\frac{m}{4}\right) 3.76 N_{2}
\end{aligned}
$$

Haciendo el balance de la ec. (86) variando x de 0 a $50 \%$ de exceso de aire se obtiene el $\% \mathrm{O}_{2}$ presente para cada valor de $\mathrm{x}$ con la siguiente ecuación:

$$
\% O_{2}=\frac{x_{G V} N_{G V}\left(n+\frac{m}{4}\right) O_{2}}{N_{G V} n C O_{2}+N_{G V} \frac{m}{2} H_{2} O+x_{G V} N_{G V}\left(n+\frac{m}{4}\right) O_{2}+N_{G V}\left(1+x_{G V}\right)\left(n+\frac{m}{4}\right) 3.76 N_{2}}
$$


Conocido el $\% \mathrm{O}_{2}$ equivalente a un $10 \%$ de aire en exceso con la ec. (87), se puede conocer cuánto vale $\varepsilon$ y $\omega$ de la ec. (85). Primero haciendo el balance de oxigeno presente y despejando $\omega$ se tiene:

$$
\begin{gathered}
\omega=\frac{2 \varepsilon N_{T}\left(n+\frac{m}{4}\right)-\left(N_{x}+N_{G V}\right)\left(2 n+\frac{m}{2}\right)}{2} \\
N_{x}=\frac{(1-\varepsilon) \dot{m}_{g c} \bar{C} p \Delta T}{M M_{c} P C I}
\end{gathered}
$$

Por lo tanto, el $\% \mathrm{O}_{2}$ presente se obtiene con:

$$
\% O_{2}=\frac{\omega O_{2}}{\left[\left(N_{x}+N_{G V}\right)+\varepsilon N_{T}\right] n C O_{2}+\left[\left(N_{x}+N_{G V}\right)+\varepsilon N_{T}\right] \frac{m}{2} H_{2} O+\varepsilon N_{T}\left(1+x_{T}\right)\left(n+\frac{m}{4}\right) 3.76 N_{2}+\omega O_{2}}
$$

Se observa que se tienen tres incógnitas y tres ecuaciones, así que se puede resolver el sistema fijando el $\% \mathrm{O}_{2}$ equivalente al $10 \%$ de exceso de aire. Con ayuda de la herramienta Solver, se obtiene el valor de $\varepsilon$, es decir, la cantidad empleada de gases de combustión proveniente de la MTG y al mismo tiempo la cantidad de combustible extra que se debe inyectar al desviar una cierta cantidad de estos gases a el medio ambiente.

Finalmente, conocido el valor de $\varepsilon$ se puede realizar el balance en la ec. (85) para cada una de los GV's de estudio empleando los gases de combustión de una MTG.

Las ec. 84 y 85 se emplean para determinar el flujo de gases de combustión al variar el exceso de aire. Con estos flujos se determina el número de Reynolds, número de Nusselt y el coeficiente convectivo de transferencia de calor del lado de los gases [55]. Además, para poder calcular estos parámetros se necesitan conocer las propiedades termo-físicas de los gases de combustión (viscosidad, conductividad térmica, capacidad calorífica y Prandtl) de forma convencional y con la inyección de los gases de la MTG.

El coeficiente de viscosidad de un gas monoatómico puro de masa molecular (MM), a la temperatura $(\mathrm{T})$, viene dado en función de los parámetros $\sigma$ y $\varepsilon$ por la siguiente ecuación:

$$
\mu=2.6693 \times 10^{-5} \frac{\sqrt{M M T}}{\sigma^{2} \Omega_{\mu}}
$$

En la que $\mu[=] \mathrm{g} \mathrm{cm}^{-1} \mathrm{seg}^{-1}, \mathrm{~T}[=] \mathrm{K}, \sigma[=] \dot{\mathrm{A}}, \varepsilon / \mathrm{k}[=] \mathrm{K}$ y $\Omega_{\mu}$ es la integral de colisión (función de colisión) es una función decreciente del número adimensional $\mathrm{kT} / \varepsilon$, cuyos valores se dan en la Tabla 29 del Apéndice A. Aunque esta fórmula se ha deducido para los gases monoatómicos, se ha encontrado que resulta también muy adecuado para los gases poliatómicos. Por otro lado, la teoría de Chapman-Enskog ha sido ampliada por Curtiss y Hirschfelder [56], para incluir las mezclas gaseosas de varios componentes. En la mayor parte de los casos resulta de gran utilidad la ecuación empírica de Wilke [57]:

$$
\mu_{\text {mezcla }}=\sum_{i=1}^{n} \frac{x_{i} \mu_{i}}{\sum_{j=1}^{n} x_{j} \phi_{i j}}
$$




$$
\phi_{i j}=\frac{1}{\sqrt{8}}\left(1+\frac{M M_{i}}{M M_{j}}\right)^{-1 / 2}\left[1+\left(\frac{\mu_{i}}{\mu_{j}}\right)^{1 / 2}\left(\frac{M M_{j}}{M M_{i}}\right)^{1 / 4}\right]^{2}
$$

Donde $n$ es el número de especies químicas existentes en la mezcla; $\mathrm{x}_{\mathrm{i}} \mathrm{y}_{\mathrm{j}}$ son las fracciones molares de las especies i y j; $\mu_{\mathrm{i}}$ y $\mu_{\mathrm{j}}$ son las viscosidades de i y j a la temperatura y presión del sistema que se obtiene a partir de la ec. (91); y $\mathrm{MM}_{\mathrm{i}}$ y $\mathrm{MM}_{\mathrm{j}}$ son las masas moleculares correspondientes. $\Phi_{\mathrm{ij}}$ es un numero adimensional y toma el valor de 1 cuando $\mathrm{i}=\mathrm{j}$. Esta aproximación de viscosidad de una mezcla gaseosa tiene una desviación del $2 \%$ con respecto a datos experimentales. En el Apéndice A se muestra el diagrama de flujo para calcular la viscosidad de los gases de combustión [57].

Para determinar la conductividad térmica de un gas volvemos a aplicar la teoría de ChapmanEnskog usada en el cálculo de la viscosidad. La fórmula de Chapman-Enskog para gases monoatómicos es la siguiente:

$$
k=1.9891 \times 10^{-4} \frac{\sqrt{T / M M}}{\sigma^{2} \Omega_{k}}
$$

En la que $\mathrm{k}[=] \mathrm{cal} \mathrm{cm}^{-1} \mathrm{seg}^{-1} \mathrm{~K}^{-1}, \sigma[=] \dot{\mathrm{A}}, \mathrm{y} \Omega_{\mathrm{k}}$ es idéntica a la función de colisión $\Omega_{\mu}$ que se empleó en la teoría de la viscosidad. En la Tabla 29 del apéndice A se dan los valores de $\Omega_{\mathrm{k}}=\Omega_{\mu}$ para el modelo potencial intermolecular de Lennard-Jones, en función del número adimensional de temperatura $\mathrm{kT} / \varepsilon$.

Para los gases poliatómicos Eucken desarrollo un método semiempírico sencillo, el cual está dado por la siguiente ecuación [58]:

$$
k=\left(\bar{C} p+\frac{5}{4} \frac{R_{u}}{M M}\right) \mu
$$

La conductividad térmica de una mezcla gaseosa se puede determinar usando de forma análoga la metodología de la viscosidad, quedando la siguiente ecuación:

$$
k_{\text {mezcla }}=\sum_{i=1}^{n} \frac{x_{i} k_{i}}{\sum_{j=1}^{n} x_{j} \phi_{i j}}
$$

Donde $\mathrm{x}_{\mathrm{i}}$ son las fracciones molares $\mathrm{y}_{\mathrm{i}}$ las conductividades de los componentes puros obtenidas usando la ec. (94). Los coeficientes $\Phi_{\mathrm{ij}}$ son los mismo que intervienen en la ecuación de la viscosidad ec. (93). En el Apéndice A se muestra el diagrama de flujo para calcular la conductividad térmica de los gases de combustión.

Para determinar la capacidad calorífica de la mezcla en función de la temperatura se utiliza la ecuación (97), donde las constantes a, b, c y d de los compuestos se obtienen de la Tabla 30 del Apéndice B

$$
\begin{aligned}
& \bar{C} p=a+b T+c T^{2}+d T^{3} \\
& \bar{C} p_{\text {mezcla }}=\sum_{i=1}^{n} \bar{C} p_{i} \frac{x_{i}}{M M_{i}}
\end{aligned}
$$


Para la estimación del número adimensional Prandtl constituye un método más sencillo, una vez conocidas las propiedades termo-físicas de la mezcla, el Prandtl se obtiene usando la fórmula de Eucken [58]:

$$
\operatorname{Pr}=\frac{\bar{C} p \mu}{k}
$$

En la Figura 36 se muestra el diagrama de flujo para el cálculo del coeficiente convectivo de los gases de combustión, en el cuál van inmersos los diagramas flujo de viscosidad y conductividad mostrados en el Apéndice A para la obtención de los números adimensionales (Re, Nu y Prandtl) los cuales son necesarios para la obtención del coeficiente convectivo.

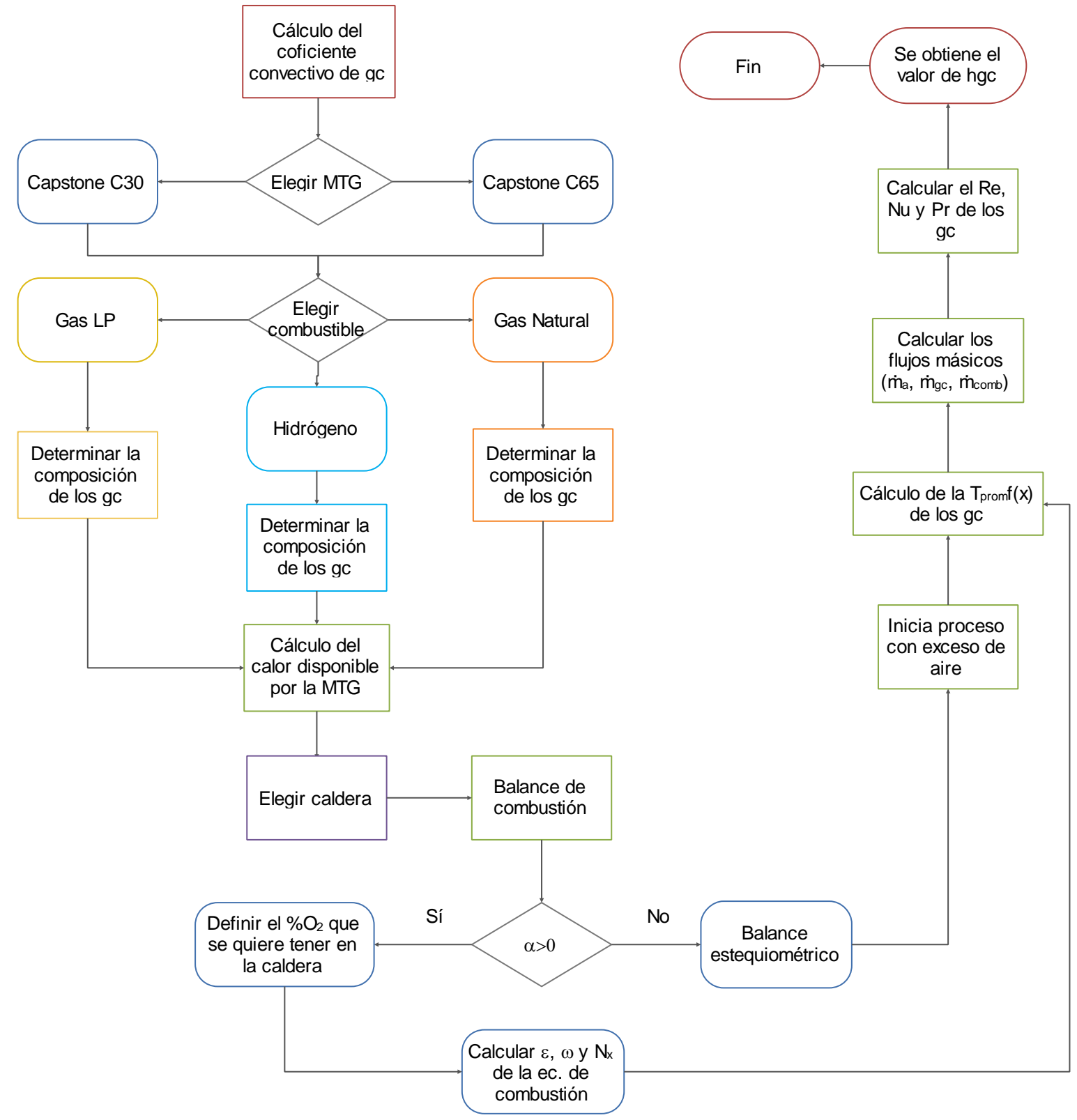

Figura 36.-Diagrama de flujo para el cálculo del coeficiente convectivo de gc 


\subsubsection{Caída de presión en el haz de tubos}

La caída de presión en el haz de tubos en principio, será mayor derivado del incremento en el flujo másico de los gases provenientes de la MTG. Estos se calculan de la misma forma que cuando opera el GV de forma convencional, como fue previamente descrito.

\subsection{Metodología de cogeneración eficiente}

La CRE evalúa el desempeño de los sistemas de cogeneración con el objeto fundamental de certificar al sistema como eficiente y a través del parámetro denominado energía libre de combustible (ELC), determinar, y en su caso, otorgar los certificados de energía limpia. Para ello desarrollo una metodología de cálculo que se presenta a continuación:

$\mathbf{E}=$ La energía eléctrica neta, medida en el punto de conexión de los generadores principales, generada en un sistema durante un año.

$\mathbf{F}=$ El combustible fósil empleado en un sistema a lo largo de un año, medido sobre poder calorífico inferior.

$\mathbf{H}=$ La energía térmica neta o el calor útil generado en un sistema y empleado en un proceso productivo durante un año.

Considerando los aspectos anteriores, el cálculo de un sistema será de la siguiente forma:

$\mathbf{R e}=$ Rendimiento eléctrico medido de un sistema, calculado como:

$$
\operatorname{Re}=\frac{E}{F}
$$

$\mathbf{R h}=$ Rendimiento térmico medio de un sistema, calculado como:

$$
\mathrm{R} h=\frac{H}{F}
$$

RefE $=$ Rendimiento de referencia para la generación eléctrica a partir de un combustible fósil en una central eficiente con tecnología actual, medido sobre la base del PCI del combustible. Se considera que la central de generación se interconecta con el SEN en media tensión.

RefH = Rendimiento de referencia para la generación térmica a partir de un combustible fósil en una central convencional eficiente de tecnología actual, medido sobre la base del PCI del combustible.

\begin{tabular}{|c|c|c|}
\hline Referencia & Capacidad de la central eléctrica (MW) & RefE \\
\hline \multirow{7}{*}{ RefE } & Capacidad $<0.5$ & $40 \%$ \\
\hline & $0.5 \leq$ Capacidad $<6$ & $44 \%$ \\
\hline & $6 \leq$ Capacidad $<15$ & $47 \%$ \\
\hline & $15 \leq$ Capacidad $<30$ & $48 \%$ \\
\hline & $30 \leq$ Capacidad $<150$ & $51 \%$ \\
\hline & $150 \leq$ Capacidad $<300$ & $52 \%$ \\
\hline & Capacidad $\geq 300$ & $53 \%$ \\
\hline \multicolumn{2}{|c|}{ RefH (con vapor o agua caliente como medio de calentamiento) } & $90 \%$ \\
\hline \multicolumn{2}{|c|}{ RefH (con uso directo de los gases de combustión) } & $82 \%$ \\
\hline
\end{tabular}

Tabla 10.- Valores de referencia 
Para las centrales eléctricas con capacidad igual o menor a $30 \mathrm{MW}$ instalados a una altura superior a $1500 \mathrm{msnm}$, generado con motores de combustión interna o con turbinas de gas, se considerarán los siguientes valores de referencia:

Tabla 11.- Valores de referencia a más de $1500 \mathrm{msnm}$

\begin{tabular}{|c|c|}
\hline Capacidad de la central eléctrica (MW) & RefE \\
\hline \hline Capacidad < 0.5 & $40 \%$ \\
\hline $0.5 \leq$ Capacidad $<6$ & $44 \%$ \\
\hline $6 \leq$ Capacidad $<15$ & $45 \%$ \\
\hline $15 \leq$ Capacidad $<30$ & $45 \%$ \\
\hline
\end{tabular}

$\mathbf{f p}=$ Factor de pérdidas de energía eléctrica debidas a la transmisión y distribución desde el nivel de alta tensión hasta el nivel de tensión al que se interconecta el sistema, calculado como:

$$
f p=1-\% \text { Pérdidas de energía eléctrica }
$$

Tabla 12.- Factor de perdida de acuerdo al nivel de tensión.

\begin{tabular}{|l|l|l|l|l|l|}
\hline Nivel de tensión & $<\mathbf{1 . 0 k V}$ & $\mathbf{1 . 0 - 3 4 . 5} \mathbf{~ k V}$ & $\mathbf{6 9 - 8 5} \mathbf{~ k V}$ & $\mathbf{1 1 5 - 2 3 0} \mathbf{~ k V}$ & $\geq \mathbf{4 0 0 k V}$ \\
\hline Factor de pérdidas & 0.910 & 0.940 & 0.960 & 0.980 & 1.000 \\
\hline
\end{tabular}

RefE' $=$ Rendimiento de referencia para la generación eléctrica a partir de un combustible fósil en una central eficiente de tecnología actual, sobre la base del PCI del combustible, medido a la tensión a la que se conecta el sistema, calculado como:

$$
\operatorname{Re} f E^{\prime}=\operatorname{Re} f E^{*} f p
$$

Fh = Combustible utilizado en el sistema atribuible a la producción de calor útil, calculado como:

$$
F h=\frac{H}{\operatorname{Re} f H}
$$

$\mathbf{F e}=$ Combustible utilizado en el sistema atribuible a la generación de energía eléctrica, calculado como:

$$
F e=F-F h
$$

$\mathbf{E E}=$ Eficiencia atribuible a la generación eléctrica calculada como:

$$
E E=\frac{E}{F \mathrm{e}}
$$

Econv $=$ Energía eléctrica generada por una central convencional eficiente, interconectada con el SEN a alta tensión, utilizando la misma cantidad de combustible que es atribuible en el sistema a la generación de energía eléctrica, calculada como:

$$
E c o n v=F e^{*} \operatorname{Re} f E
$$

$\mathbf{E P}=$ Energía primaria obtenida del análisis por separado del comportamiento del proceso de generación de energía eléctrica y del proceso térmico del sistema, calculado como:

$$
E P=\frac{E}{\operatorname{Re} f E^{\prime}}+\frac{H}{\operatorname{Re} f H}
$$


AEP = Ahorro de energía primaria, obtenida del análisis por separado del comportamiento del proceso de generación de energía eléctrica y del proceso térmico del sistema, calculado como:

$$
A E P=E P-F
$$

APEP = Ahorro porcentual de energía primaria obtenida del análisis por separado del comportamiento del proceso de generación de energía eléctrica y del proceso térmico del sistema, calculado como:

$$
A P E P=\frac{E P-F}{E P}
$$

AREL = El aporte relativo de energía limpia de una central eléctrica de cogeneración con relación a la energía generada por la central de referencia, calculado de la siguiente forma:

$$
A R E L=\frac{A E P}{F e}
$$

ELC = Energía libre de combustible, esto es, la energía eléctrica generada en la central eléctrica de cogeneración por encima de la que se generaría en una central térmica, utilizando la misma cantidad de combustible que en una central eléctrica de cogeneración (MWh), calculada como:

$$
E L C=A E P * \operatorname{Re} f E
$$

El porcentaje de energía libre de combustible se determinará conforme a lo siguiente:

$$
\% E L C=\frac{E L C}{E}
$$

\subsection{Cálculo de las emisiones de $\mathrm{CO}_{2}$}

Es importante conocer las emisiones de $\mathrm{CO}_{2}$ debido a que el sector de generación de energía aporta aproximadamente el $18 \%$ del total. De acuerdo con la LGCC, México tiene como meta reducir la emisión de los GEI en un 30\% para el 2020 y en un 50\% para el 2050 respecto a las emitidas en el 2000 [8]. Para cumplir con dicha meta es necesario usar los combustibles fósiles con la mayor eficiencia posible.

El cálculo de las emisiones de $\mathrm{CO}_{2}$ se hace primero para los sistemas convencionales y posteriormente para el sistema de MCHP.

\subsubsection{Micro-Turbina de Gas}

A partir de la metodología planteada en el capítulo 6 se obtiene el flujo de combustible que requiere cada una de las MTG's de estudio. A partir de ese flujo se obtienen los moles de combustible, al hacer el balance de la ecuación de combustión en funciones de los moles de combustible (ec. 79) se obtienen los moles de $\mathrm{CO}_{2}$, al multiplicar por la $\mathrm{MM}$ del $\mathrm{CO}_{2}$ se obtienen los kilogramos de $\mathrm{CO}_{2}$. Considerando que opera 8,390 horas al año, se hace la conversión de kg/s a toneladas/año de $\mathrm{CO}_{2}$ :

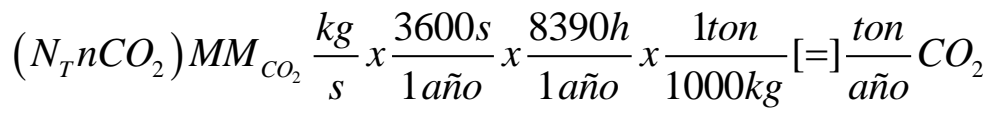

Una vez ubicada la MTG se obtiene la potencia real que entrega y al multiplicarla por el número de horas que trabaja al año se obtienen los MWh/año: 


$$
\dot{W}_{T} k W x \frac{8390 h}{1 a \tilde{n} o} \times \frac{1 M W}{1000 k g}[=] \frac{M W h}{a \tilde{n} o}
$$

Finalmente, al hacer el cociente de la ecuación 114 y 115 se obtienen las toneladas anuales de $\mathrm{CO}_{2}$ emitidas por cada MWh generado.

\subsubsection{Generador de vapor}

Siguiendo el mismo procedimiento del caso anterior se obtienen las toneladas anuales de $\mathrm{CO}_{2}$. Con la potencia del GV se calcula la energía térmica generada (MWht) con la ec. (115).

\subsubsection{MCHP}

El consumo de combustible a emplear para el sistema de MCHP se obtiene con la ecuación (81), donde se incluye los gases de combustión provenientes de la MTG. El cálculo de los moles de CO2 se obtienen al hacer el balance en la ecuación (84). Finalmente, para el cálculo de la energía eléctrica y térmica totales (MWh/año) solo se suman ambas potencias y se multiplica por el número de horas anuales que trabaje el sistema. 


\section{Capítulo 7. Resultados}

\subsection{Micro-Turbina de Gas}

Como se desconocen $\pi$ y la temperatura de entrada a la turbina (TET), se realizó la iteración de 3 variables en el intervalo que se muestra en la Figura 37, que son: trabajo motor, temperatura de entrada de los gases y la relación de presiones. Con estas variaciones se encontró que la turbina C30 opera con una $\pi=3.2$ y TET $=1050{ }^{\circ} \mathrm{C}$ utilizando como combustible Gas Natural, ya que a esas condiciones se obtiene la potencia reportada por el fabricante y el calor disponible para proceso reportado en la literatura[59][60].

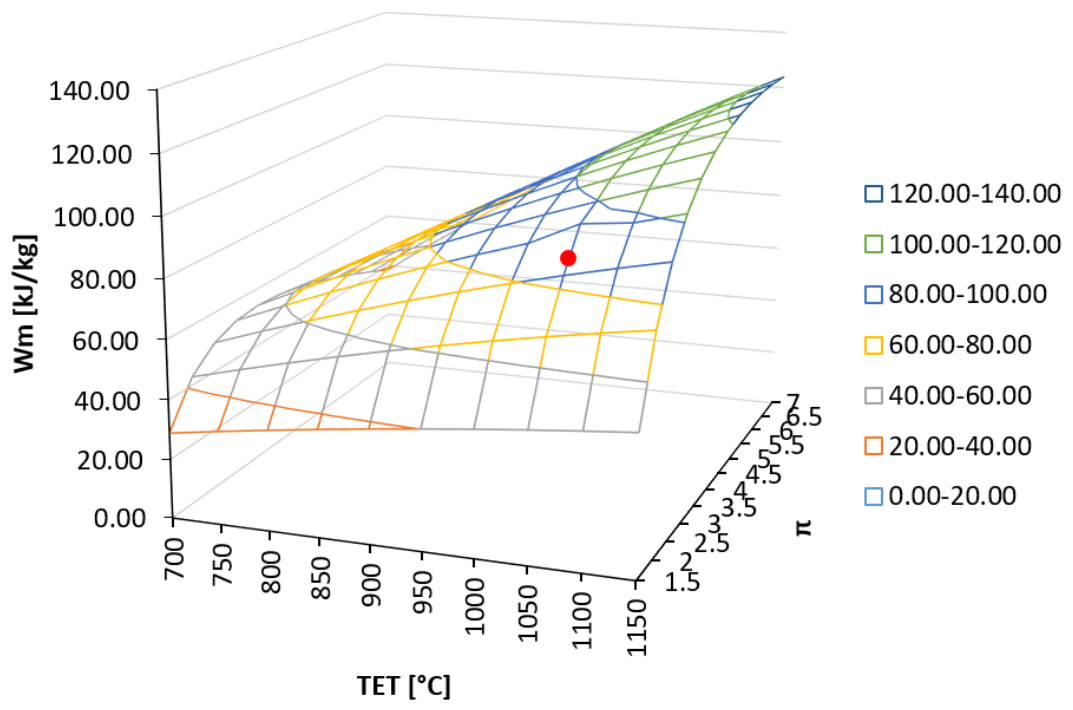

Figura 37.- Sabana micro-turbina Capstone C30

Una vez conocidos $\pi$ y TET, se obtiene el coeficiente de derrateo. En la Figura 38, se muestra el comportamiento de la MTG C30 variando las condiciones atmosféricas (T, P), se observa que tiene un decremento en la potencia del $5.52 \%$ por cada $500 \mathrm{msnm}$ y del $3.95 \%$ por cada $5^{\circ} \mathrm{C}$ que aumenta la temperatura.

En el caso de la MTG C65 al igual que en la MTG C30 se desconocen $\pi$ y TET, se realizó la iteración de 3 variables como se muestra en la Figura 39. Se encontró que la turbina C65 opera con una $\pi=3.7$ y $\mathrm{TET}=1200^{\circ} \mathrm{C}$, ya que a esas condiciones se obtiene la potencia reportada por el fabricante y el calor disponible para proceso reportado en la literatura [60].

Una vez conocidos $\pi$ y TET, se obtiene el coeficiente de derrateo. En la Figura 40, se muestra el comportamiento de la MTG C65 variando las condiciones atmosféricas (T, P), se observa que tiene un decremento en la potencia del $6.32 \%$ por cada $500 \mathrm{msnm}$ y del $3.86 \%$ por cada $5^{\circ} \mathrm{C}$ que aumenta la temperatura. 


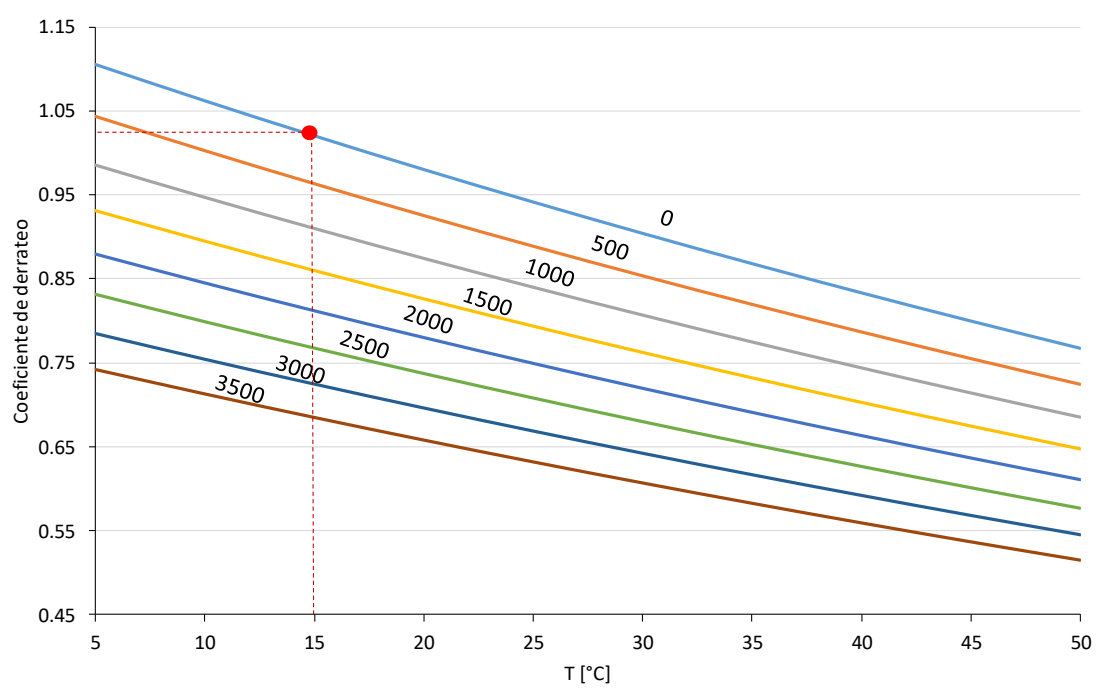

Figura 38.- Derrateo micro-turbina Capstone C30

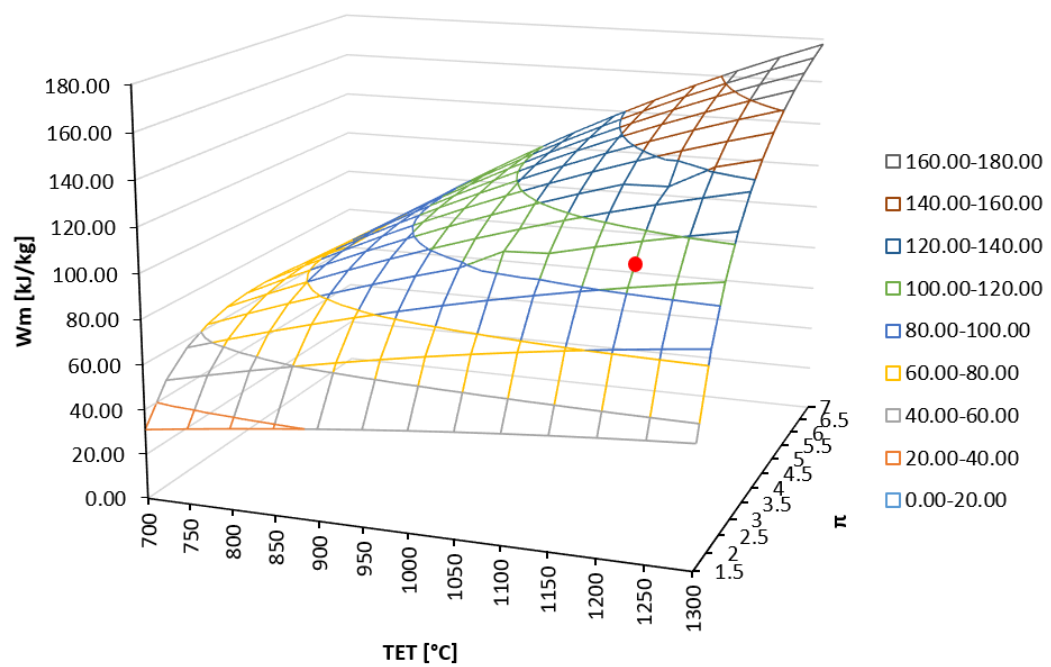

Figura 39.- Sabana micro-turbina Capstone C65

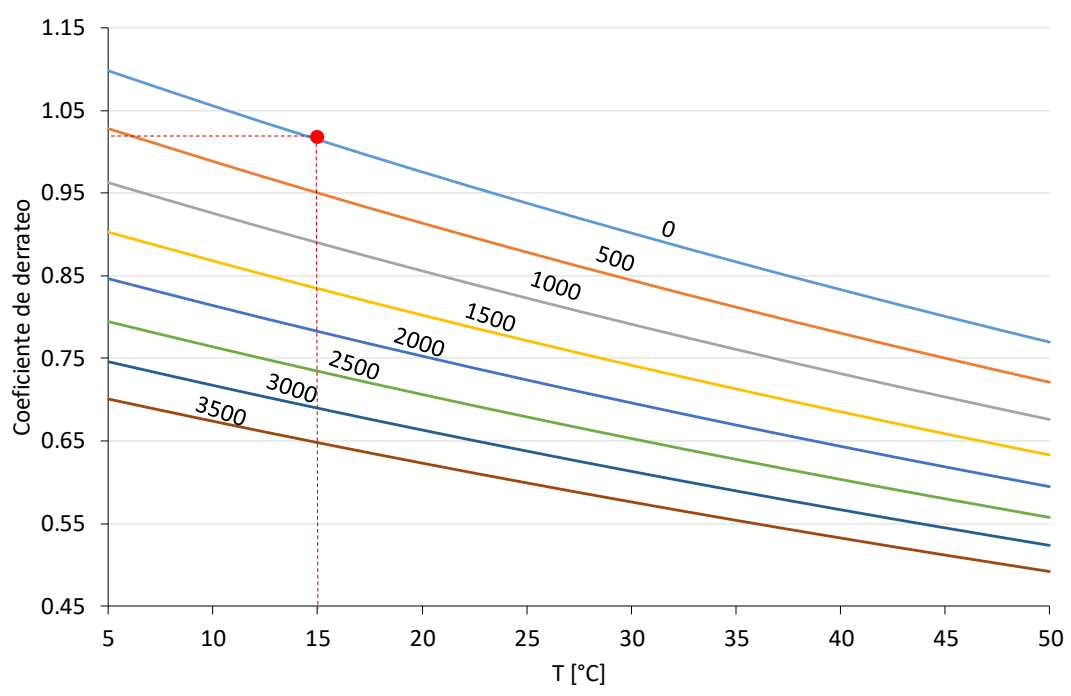

Figura 40.- Derrateo micro-turbina Capstone C65 
De igual forma se obtuvieron los resultados empleando Gas LP e hidrógeno. Como las curvas son muy similares, en las Tabla 13 y Tabla 14 se hace el comparativo para las turbinas C30 y C65. Para poder determinar la variación de potencia que entrega la turbina y en consecuencia el flujo de gases a la salida, se debe conocer las condiciones geográficas y climáticas que prevalecen en el sitio de instalación del sistema. Por ejemplo, si seleccionamos la CDMX, esta se encuentra ubicada a 2250 msnm y una temperatura media anual de $25^{\circ} \mathrm{C}$, se encontrará un decremento en la potencia de la C30 y C65 de: $27.7 \%$ y $30.38 \%$ respectivamente. Para el calor disponible se verá que, para el hidrógeno, a pesar de decaer a condiciones in situ, este sigue siendo elevado y aprovechable para el proceso.

Tabla 13.- Resultados con los 3 combustibles en condiciones ISO

\begin{tabular}{|c|c|c|c|c|c|c|}
\hline \multirow{2}{*}{$\begin{array}{l}\text { Altura }=0 \mathrm{msnm} \text { y } \\
\text { Tambiente }=15^{\circ} \mathrm{C}\end{array}$} & \multicolumn{3}{|c|}{$\mathbf{C 3 0}$} & \multicolumn{3}{|c|}{ C65 } \\
\hline & GN & GLP & H2 & GN & GLP & H2 \\
\hline$\dot{\mathrm{m}}_{\text {comb }}[\mathrm{kg} / \mathrm{s}]$ & 0.0048 & 0.0050 & 0.0008 & 0.0085 & 0.0090 & 0.0014 \\
\hline$\dot{\mathrm{m}}_{\text {aire }}[\mathrm{kg} / \mathrm{s}]$ & 0.30764 & 0.30750 & 0.29304 & 0.48541 & 0.48514 & 0.45332 \\
\hline Exceso de aire $\mathrm{x}[\%]$ & 277.43 & 292.40 & 953.80 & 233.45 & 246.64 & 820.20 \\
\hline $\mathrm{Q}_{\text {disp }}[\mathrm{kW}]$ & 66.43 & 64.34 & 76.63 & 121.92 & 118.09 & 152.07 \\
\hline $\mathrm{Q} / \mathrm{E}$ & 2.2 & 2.2 & 2.46 & 1.9 & 1.8 & 2.36 \\
\hline Eficiencia [\%] & 29.69 & 29.69 & 26.61 & 35.09 & 35.09 & 27.56 \\
\hline
\end{tabular}

Tabla 14.- Resultados con los 3 combustibles en condiciones atmosféricas de la CDMX

\begin{tabular}{|l|c|c|c|c|c|c|}
\hline \multirow{2}{*}{$\begin{array}{c}\text { Altura } \\
\text { Tambiente }=25 \mathbf{2 5}^{\circ} \mathbf{C}\end{array}$} & \multicolumn{3}{|c|}{ C30 } & \multicolumn{3}{c|}{ C65 } \\
\cline { 2 - 7 } & GN & GLP & H2 & GN & GLP & H2 \\
\hline$\dot{\mathrm{m}}_{\text {comb }}[\mathrm{kg} / \mathrm{s}]$ & 0.0035 & 0.0037 & 0.0006 & 0.0062 & 0.0066 & 0.0011 \\
\hline$\dot{\mathrm{m}}_{\text {aire }}[\mathrm{kg} / \mathrm{s}]$ & 0.23064 & 0.23053 & 0.21969 & 0.36391 & 0.36370 & 0.33985 \\
\hline Exceso de aire x $[\%]$ & 285.63 & 300.93 & 962.49 & 242.75 & 256.31 & 827.03 \\
\hline $\mathrm{Q}_{\text {disp }}[\mathrm{kW}]$ & 52.72 & 51.06 & 59.96 & 96.23 & 93.21 & 118.03 \\
\hline Q/E & 2.5 & 2.4 & 2.68 & 2.2 & 2.1 & 2.54 \\
\hline Eficiencia $[\%]$ & 28.54 & 28.54 & 25.71 & 32.66 & 32.66 & 26.75 \\
\hline
\end{tabular}

\subsection{Generador de vapor}

En la Tabla 15 se muestran los moles de oxigeno $(\alpha)$ contenidos en los gases efluentes de cada MTG para ser empleados en cada GV de estudio (sin exceso de aire). Se muestra que ambas MTG's pueden abastecer la gama de GV's de estudio, sin embargo, para que la eficiencia del sistema sea la máxima posible, $\alpha$ tiene que ser menor a cero. Esto quiere decir que requiere de oxigeno adicional y se pueden emplear en su totalidad los gases efluentes de la MTG. Cuando $\alpha$ es mayor a cero, al tener un exceso de oxigeno se tiene que desviar una parte del flujo de gases de la MTG, disminuyendo así la eficiencia del sistema. Por lo tanto, se buscaría acoplar los gases efluentes de la MTG a un GV en la que $\alpha$ sea menor a cero. 
Tabla 15.- Evaluación de a para cada MTG y GV de estudio

\begin{tabular}{|c|c|c|c|c|c|c|}
\hline \multirow{2}{*}{$\mathbf{G V}$} & \multicolumn{3}{|c}{ C30 } & \multicolumn{3}{c|}{ C65 } \\
\cline { 2 - 8 } & $\mathbf{G N}$ & $\mathbf{G L P}$ & $\mathbf{H 2}$ & $\mathbf{G N}$ & $\mathbf{G L P}$ & H2 \\
\hline $\mathbf{1 5} \mathbf{~ C B}$ & 0.00094 & 0.00097 & 0.00131 & 0.00170 & 0.00173 & 0.00224 \\
\hline $\mathbf{2 0} \mathbf{~ C B}$ & 0.00079 & 0.00082 & 0.00118 & 0.00155 & 0.00158 & 0.00212 \\
\hline $\mathbf{3 0} \mathbf{~ C B}$ & 0.00049 & 0.00053 & 0.00094 & 0.00125 & 0.00129 & 0.00187 \\
\hline $\mathbf{4 0} \mathbf{C B}$ & 0.00019 & 0.00024 & 0.00069 & 0.00094 & 0.00100 & 0.00162 \\
\hline $\mathbf{5 0} \mathbf{C B}$ & -0.00011 & -0.00005 & 0.00044 & 0.00064 & 0.00071 & 0.00137 \\
\hline $\mathbf{6 0} \mathbf{C B}$ & -0.00042 & -0.00034 & 0.00019 & 0.00034 & 0.00042 & 0.00113 \\
\hline $\mathbf{7 0} \mathbf{C B}$ & -0.00068 & -0.00060 & -0.00002 & 0.00007 & 0.00016 & 0.00091 \\
\hline $\mathbf{8 0} \mathbf{C B}$ & -0.00098 & -0.00088 & -0.00027 & -0.00022 & -0.00012 & 0.00067 \\
\hline $\mathbf{1 0 0} \mathbf{~ C B}$ & -0.00157 & -0.00145 & -0.00075 & -0.00081 & -0.00069 & 0.00018 \\
\hline
\end{tabular}

Cuando se tiene un valor de $\alpha>0$, se buscaría desviar el menor flujo posible, ya que al tener un exceso de $\mathrm{O}_{2}$ elevado, se puede determinar la cantidad de flujo a desviar fijando un porcentaje de $\mathrm{O}_{2}$ deseado en el GV como se mostró en el capítulo 6. En las Figura 41 y 42 se muestra el comportamiento de $\alpha$ en función de la gama de calderas empleando la MTG C30 y C65 respectivamente. Además, se muestra el valor de $\alpha$ de forma porcentual $\left(\% \mathrm{O}_{2}\right)$ para ser comparado con un porcentaje de $\mathrm{O}_{2}$ equivalente al $10 \%$ de exceso de aire, con el cual normalmente operan los GV's. Por lo tanto, todas las calderas que están por arriba de la línea roja $\left(\% \mathrm{O}_{2}\right.$ equivalente al $10 \%$ de exceso de aire), se tiene que desviar una parte de los gases efluentes de la MTG según sea el caso.

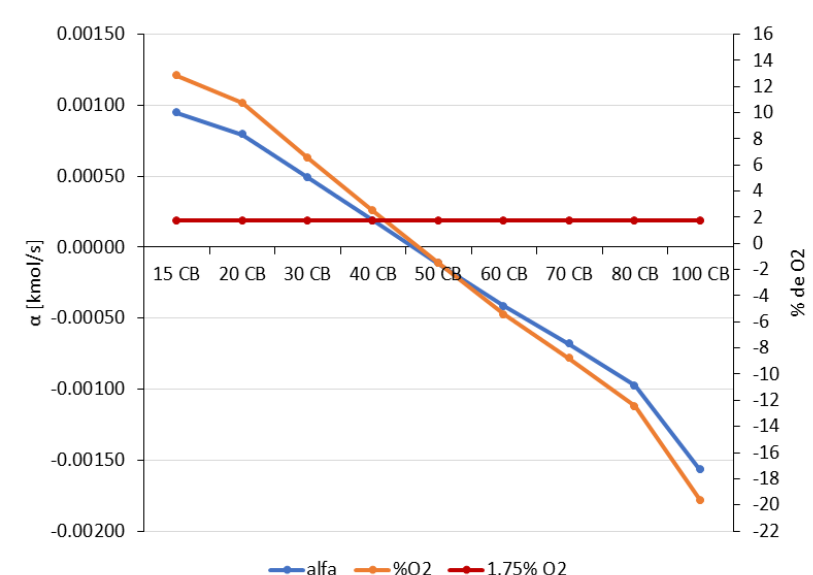

Figura 41.- Alfa en función de los GV's de estudio y $\%$ de $\mathrm{O}_{2}$ en los gases de la MTG C30

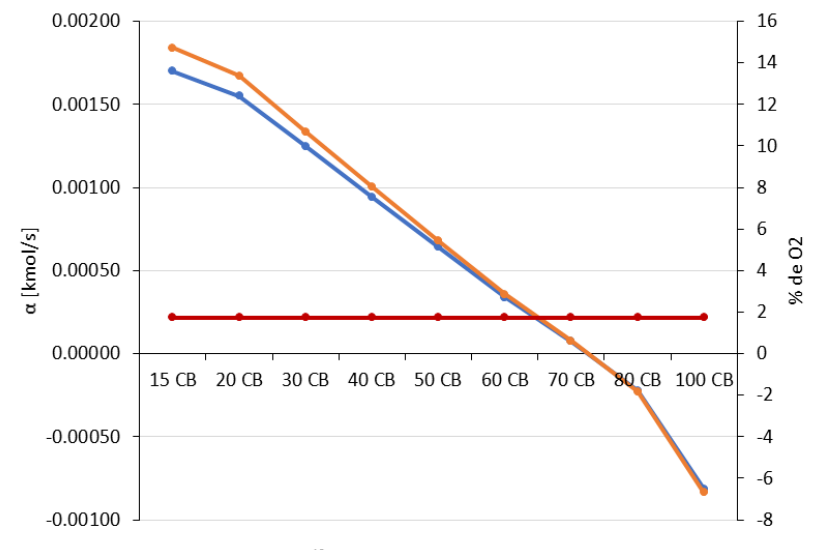

$\rightarrow$ alfa $\rightarrow \% 02 \rightarrow 1.75 \% 02$

Figura 42.- Alfa en función de los GV's de estudio y \% de $\mathrm{O}_{2}$ en los gases de la MTG C65

A modo de ejemplo, se eligió el GV 80CB, este debido a que con ambas MTG's $\alpha<0$ y se puede utilizar todo el calor disponible de los gases de escape. Con la finalidad de comparar la viabilidad de acoplar una MTG a un GV que requiere de mayor cantidad de aire que la proveniente de la turbina. Para poder hacer una comparación del GV con las MTG's, se obtiene el flujo de gases de combustión y el coeficiente convectivo de forma convencional, los cuales se muestra en las Figura 43 y 44 respectivamente, usando como combustible GN, GLP e $\mathrm{H}_{2}$.

En la Figura 43 se observa que los gases de combustión a medida que se incrementa el exceso de aire estos también se incrementan, puesto que se está inyectando una mayor cantidad de aire. Además, se observa que el flujo es mayor con GN. Debido a que la $\mathrm{MM}_{\mathrm{GLP}}>\mathrm{MM}_{\mathrm{GN}}$, implica que el número de moles de combustible que requiere el $\mathrm{GV}$ sea $\mathrm{Na}_{\mathrm{GN}}>\mathrm{Na}_{\mathrm{GLP}}$. Para el caso del hidrógeno el flujo de gases de combustión es menor debido a su PCI es mayor. Implica que se usa menor 
combustible, aunque con un elevado exceso de aire y, por ende, el coeficiente convectivo es menor en comparación con el del GN y GLP.

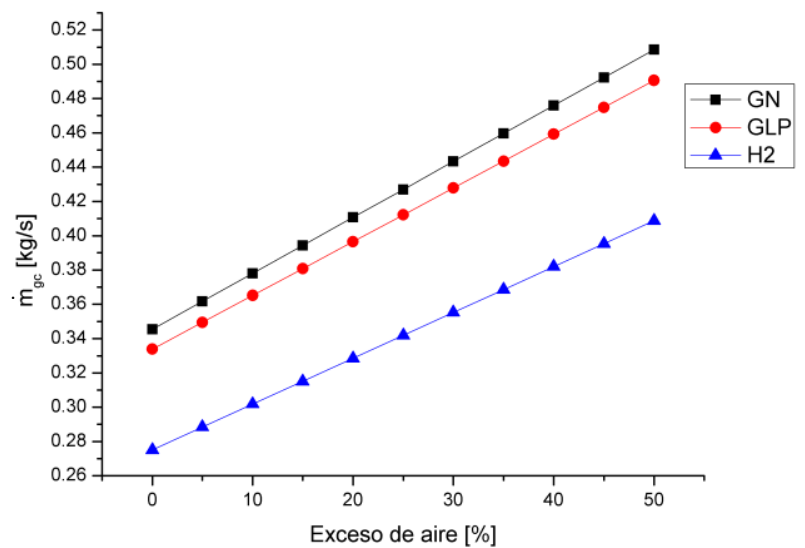

Figura 43.- Flujo de gc del GV 80CB convencional con $G N, G L P$ e $\mathrm{H}_{2}$ en función del exceso de aire.

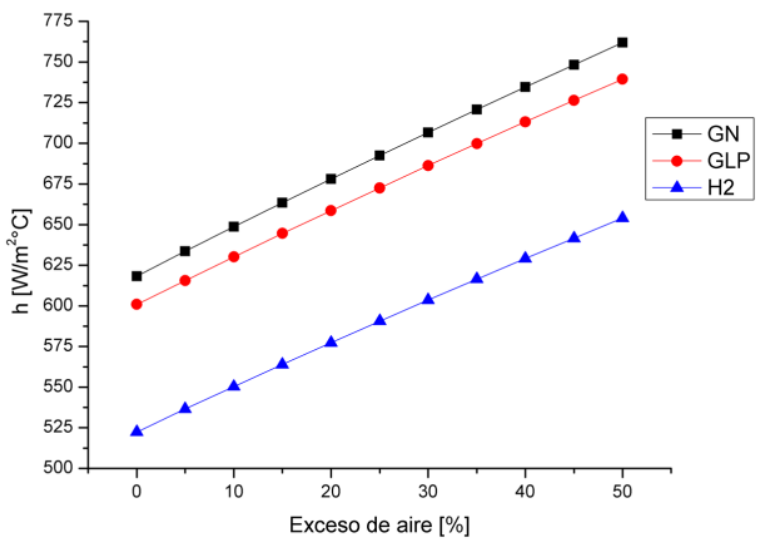

Figura 44.- Coeficiente convectivo del GV 80CB convencional con $\mathrm{GN}$, GLP e $\mathrm{H}_{2}$ en función del exceso de aire.

\subsection{Acoplamiento de gases de combustión en un GV}

Antes de hacer el acoplamiento de los gases provenientes de una MTG a un GV convencional, es necesario hacer un análisis de sensibilidad, para ver qué parámetros son los de mayor impacto en el comportamiento del coeficiente convectivo y así poder dar una mejor interpretación de los resultados obtenidos.

Puesto que el coeficiente convectivo es la facilidad de transferir calor de un medio a otro, el número de Nusselt (ec. 116), lo define en función de la transferencia de calor por convección y conducción. Este a su vez es función del número de Reynolds (ec. 117), que relaciona las fuerzas inerciales y las viscosas. El Reynolds define el régimen del flujo del fluido: laminar, transitorio o turbulento. Para cada régimen existen correlaciones empíricas para calcular el Nusselt al que se le incluye el número de Prandtl (ec. 118), que relaciona la difusividad de momento y de calor.

$$
\begin{aligned}
N u & =\frac{h D}{k} \\
\operatorname{Re} & =\frac{v D}{v}=\frac{\rho v D}{\mu} \\
\operatorname{Pr} & =\frac{v}{\alpha}=\frac{C p \mu}{k} \\
\text { Donde: } \quad \alpha & =\frac{k}{\rho C p} \quad v=\frac{\mu}{\rho}
\end{aligned}
$$

El coeficiente de transferencia de calor por convección global interna es una función de $\mathrm{Nu}$, Cp y k. La viscosidad y la capacidad calorífica son función de la temperatura. Debido a que la temperatura es inversamente proporcional al exceso de aire, el coeficiente convectivo depende de la pendiente de k y Nu y ésta podría ser positivo o negativo. Si la pendiente de $\mathrm{Nu} \gg \mathrm{k}$ la pendiente del coeficiente convectivo será positiva e inversamente. En el Apéndice C se encuentra el desarrollo detallado de ese comportamiento. 
Una vez que se conoce la variación de flujo de gases en función del exceso de aire y del tipo de combustible empleado en el GV ya sea que ésta opere de manera convencional o con los gases provenientes de la MTG (Figura 45 a 48), resulta que al emplear los gases efluentes de la MTG en el $\mathrm{GV}$ el flujo de gases de escape del sistema es mayor al empleado convencionalmente. Si el régimen de operación se mantiene laminar y, en el caso limite el coeficiente convectivo se mantiene constante, el tiempo de residencia de los gases en los tubos se reduce y la temperatura de estos en la chimenea se incrementará.

En cuanto a el combustible empleado, se tiene un mayor flujo de gases de combustión usando GN. Tal como se mencionó anteriormente, debido a que la $\mathrm{MM}_{\mathrm{GLP}}>\mathrm{MM}_{\mathrm{GN}}$, implica que el número de moles de combustible que requiere el $\mathrm{GV}$ tenga un comportamiento inverso: $\mathrm{Na}_{\mathrm{GN}}>\mathrm{Na} \mathrm{GLP}_{\mathrm{GL}}$

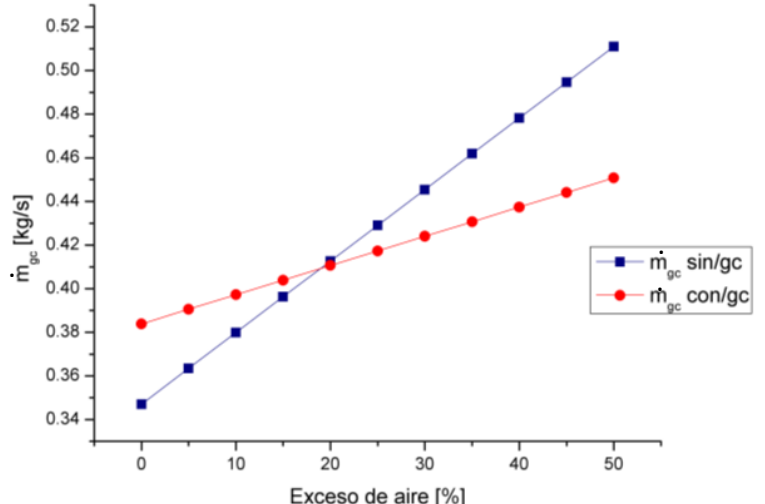

Figura 45.- Flujo de gc del GV 80CB con y sin gases provenientes de la MTG C30 con GN.

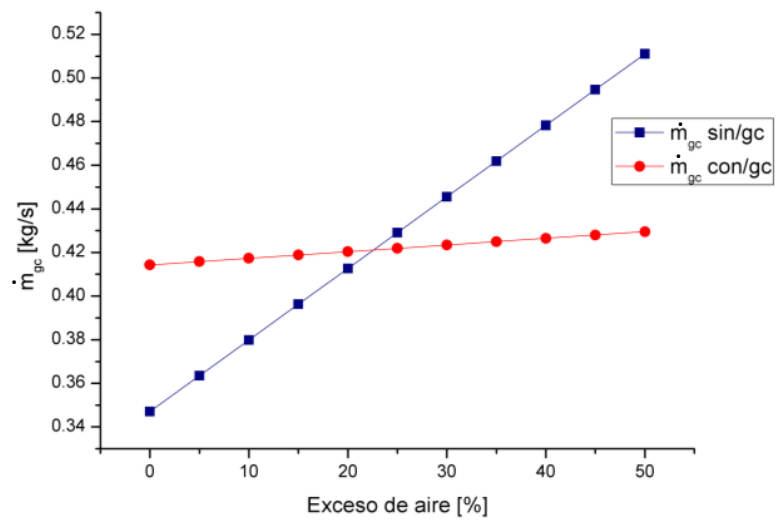

Figura 47.- Flujo de gc del GV 80CB con y sin gases provenientes de la MTG C65 con GN.

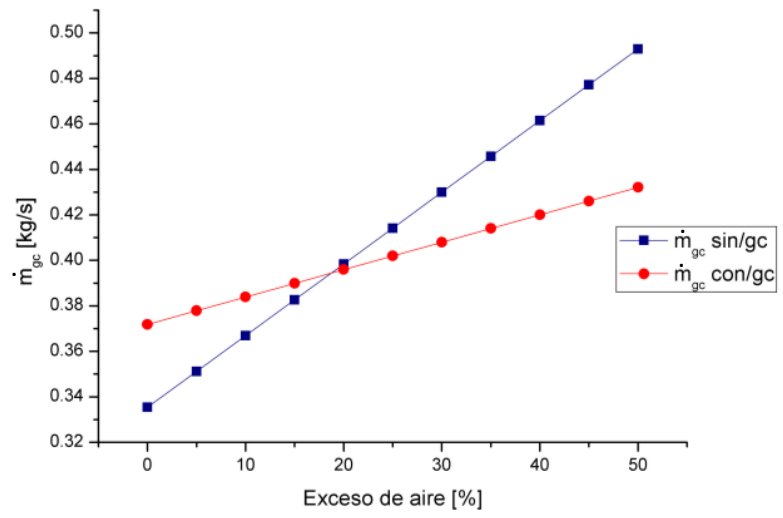

Figura 46.- Flujo de gc del GV 80CB con y sin gases provenientes de la MTG C30 con GLP.

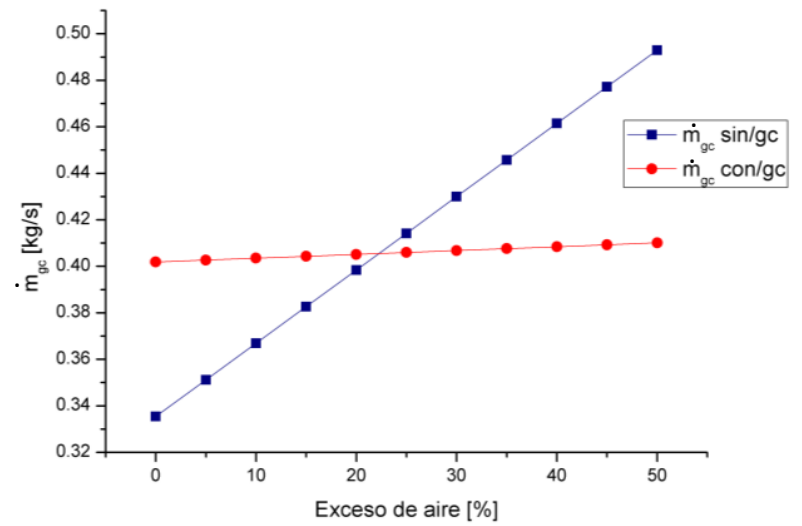

Figura 48.- Flujo de gc del GV 80CB con y sin gases provenientes de la MTG C65 con GLP

Para el caso del hidrógeno se observa que la MTG C30 tiene un flujo de gases de combustión menor al empleado de forma convencional por el GV bajo estudio (Figura 49). Esto se debe al elevado PCI del hidrógeno que es 2.17 veces mayor al del GN y 2.6 veces mayor del GLP. Por consiguiente, el flujo que requiere de combustible es menor, aunque el exceso de aire es mayor. En el caso de la MTG C65 se observa lo contrario (Figura 50). En la Tabla 15 se mostró los GV's que cubren cada una de estas MTG's sin variar el exceso de aire y ambas MTG's cubre las necesidades del GV bajo estudio. Al disminuir el flujo de combustible empleando hidrógeno, los gases de combustión siguen siendo mayor. Si se evaluara la MTG C65 con otro GV más grande obtendríamos el mismo comportamiento que con la MTG C30. 


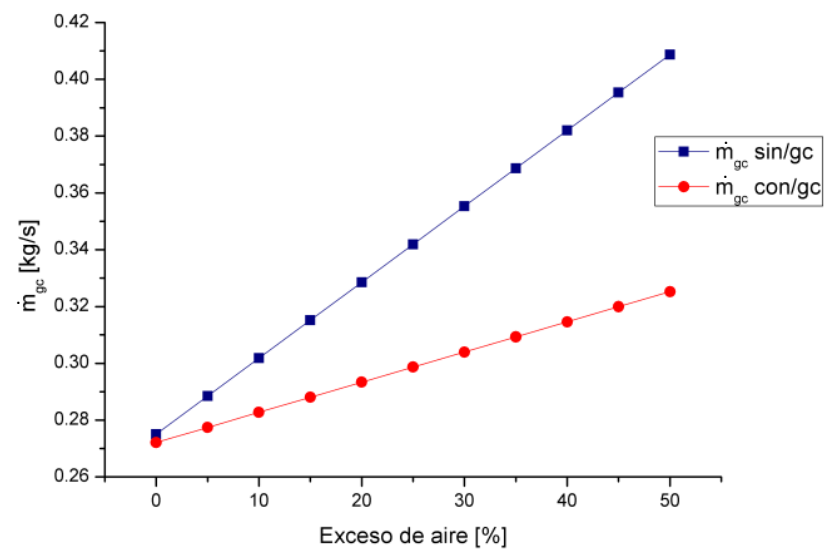

Figura 49.- Flujo de gc del GV 80CB con y sin gases provenientes de la MTG C30 conH2

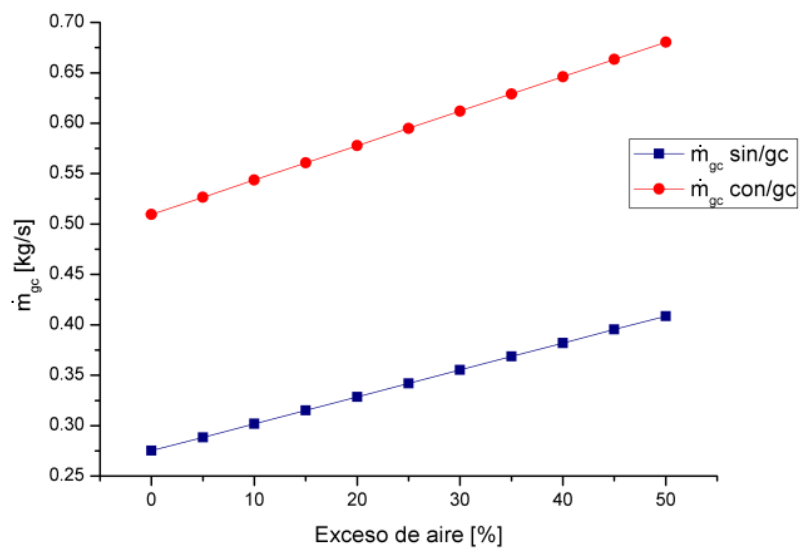

Figura 50.- Flujo de gc del GV 80CB con y sin gases provenientes de la MTG C65 conH2

Para corroborar si el comportamiento del GV se ve afectado al usar los gases provenientes de la MTG, se evalúa el coeficiente convectivo de transferencia de calor para ambos casos (Figura 51 a 54). El coeficiente convectivo de transferencia de calor será mayor cuando se suministran los gases efluentes de las MTG's C30 y C65; en un promedio de 13 y 18\% respectivamente en función del combustible empleado. La diferencia en el incremento de $\mathrm{h}_{1}$ se reduce en función del incremento en el exceso de aire. Es de notarse que un GV que opera con GN o GLP difícilmente llegará a tener un exceso de aire mayor al 20\%. De esta manera se demuestra la conveniencia del empleo de los gases de combustión de la MTG en un GV convencional.

Además, se observa que usando la MTG C30 la pendiente del coeficiente convectivo es positiva debido a que la pendiente del $\mathrm{Nu}>>$ k. Para el caso de la MTG C65 la predominancia es de la conductividad térmica sobre el Nusselt y de ahí que su pendiente negativa.

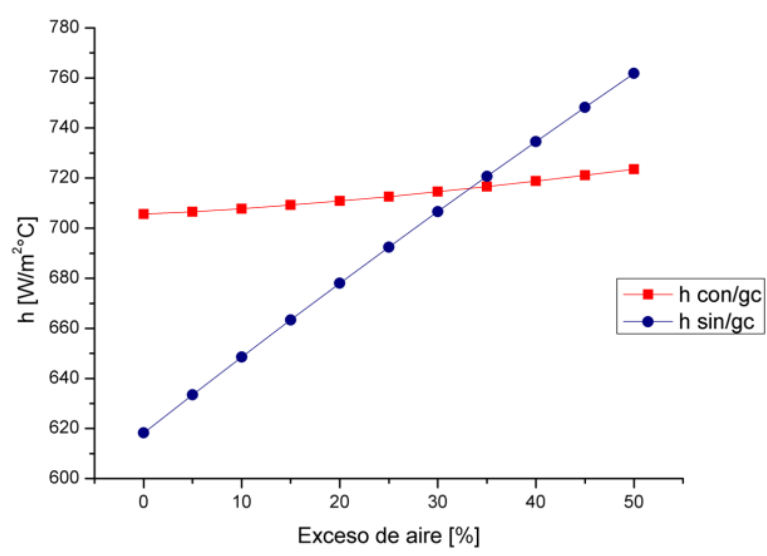

Figura 51.- Coeficiente convectivo del GV $80 C B$ con y sin gases provenientes de la MTG C30 con GN.

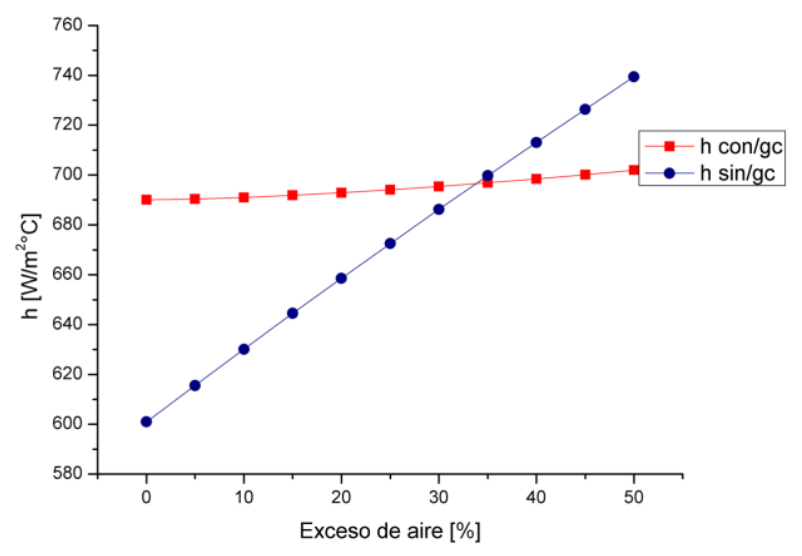

Figura 52.- Coeficiente convectivo del GV $80 C B$ con y sin gases provenientes de la MTG C30 con GLP. 


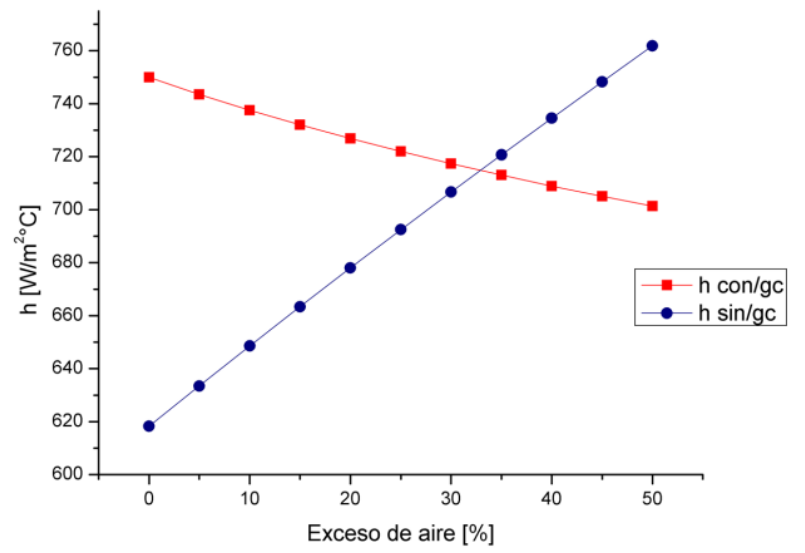

Figura 53.- Coeficiente convectivo del GV 80CB con y sin gases provenientes de la MTG C65 con GN.

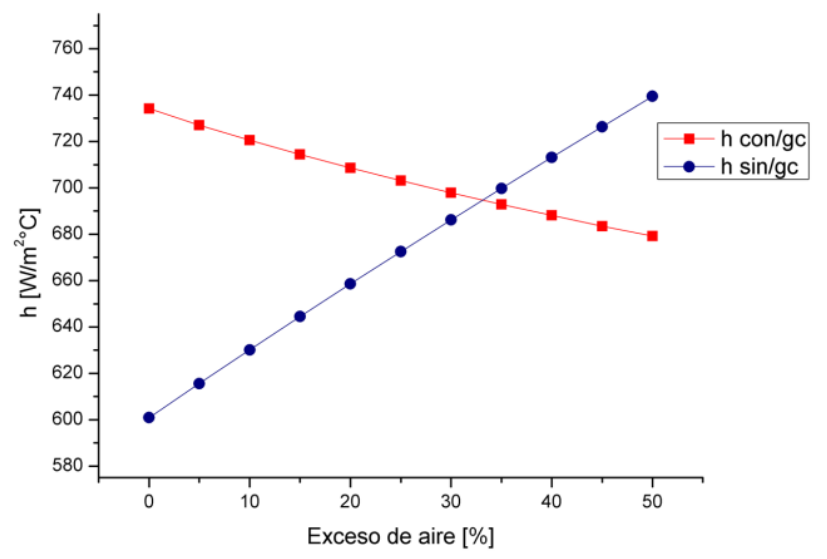

Figura 54.- Coeficiente convectivo del GV $80 C B$ con y sin gases provenientes de la MTG C65 con GLP.

Para el caso del hidrógeno el coeficiente convectivo es menor empelando la MTG C30 que la C65, debido a que el flujo de gases de combustión es menor que el requerido de forma convencional. Al no tener un cambio de régimen el coeficiente decrece (Figura 55). Para el caso de la MTG C65 que tuvo un incremento en el flujo de gases de combustión, el coeficiente convectivo tiene un incremento promedio del 9\% (Figura 56).

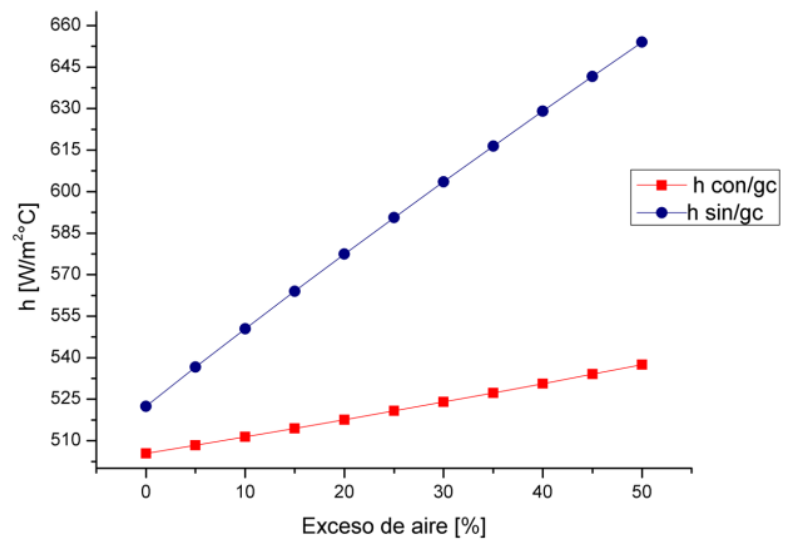

Figura 55.- Coeficiente convectivo del GV $80 C B$ con y sin gases provenientes de la MTG C3O con $\mathrm{H}_{2}$

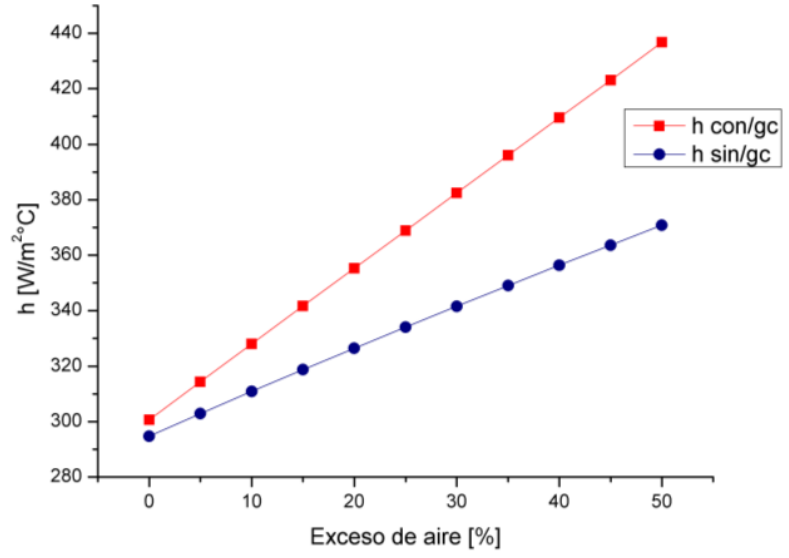

Figura 56.- Coeficiente convectivo del GV $80 C B$ con y sin gases provenientes de la MTG C65 con $\mathrm{H}_{2}$

En cuanto al coeficiente convectivo del lado del agua, al ser las condiciones de vapor constantes, el coeficiente no tiene gran cambio al variar el exceso de aire. En las Figura 57 y 58 se muestra el comportamiento del coeficiente convectivo $h_{2}$ comparado con $h_{1}$ para cada turbina y en función de la gama de GV's de estudio. Debido a que $\mathrm{h}_{2}$ es constante, independientemente de la MTG que se emplee para inyectar sus gases efluentes a un GV, este no cambia. Además, como es mucho mayor que el coeficiente $h_{1}$, este no tiene una gran resistencia para el cálculo de $\mathrm{U}$, por lo tanto, el considerarlo constante no afecta de manera sustancial al coeficiente global U. 


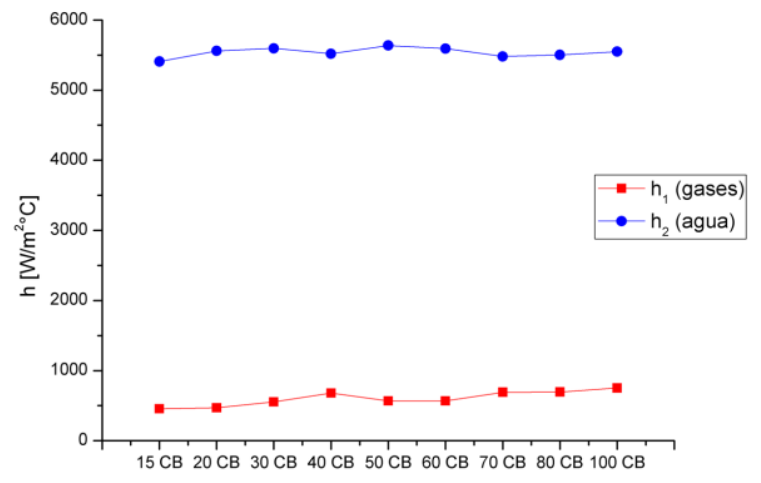

Figura 57.- Coeficientes convectivos $h_{1}$ y $h_{2}$ para cada $G V$ de estudio con los gases provenientes de la MTG C30 con $G N$.

Conocidos los coeficientes de transferencia de calor de lado de los gases y del agua, se determinó el coeficiente global de transferencia de calor. Como ya se mencionó, el coeficiente convectivo del lado de los gases es mucho menor que al del lado del agua, éste presenta la mayor resistencia en la obtención del coeficiente global. De esta manera, los valores obtenidos para U son cercanos al de $h_{1}$ como se muestra en las Figura 59 a 64 . Al ser su comportamiento muy cercano al de $\mathrm{h}_{1}$, las pendientes e incrementos o decrementos según sea el caso son debido a los mismos fenómenos con operan sobre $\mathrm{h}_{1}$.

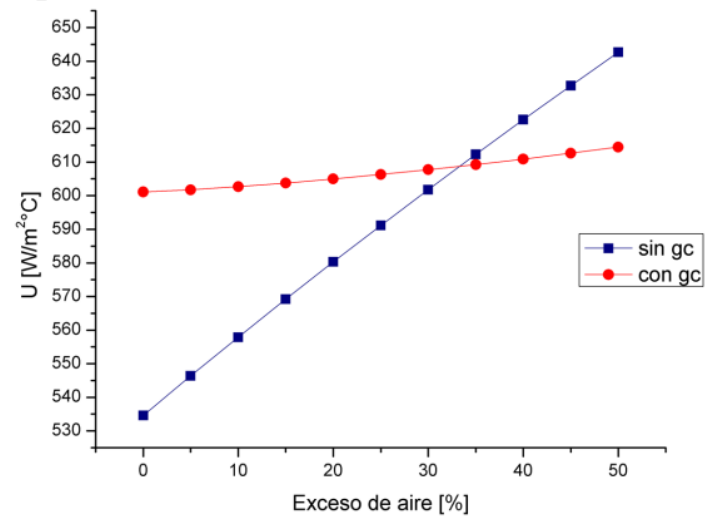

Figura 59.- Coeficiente global de transferencia de calor del GV 80CB con y sin gases provenientes de la MTG C30 con $G N$

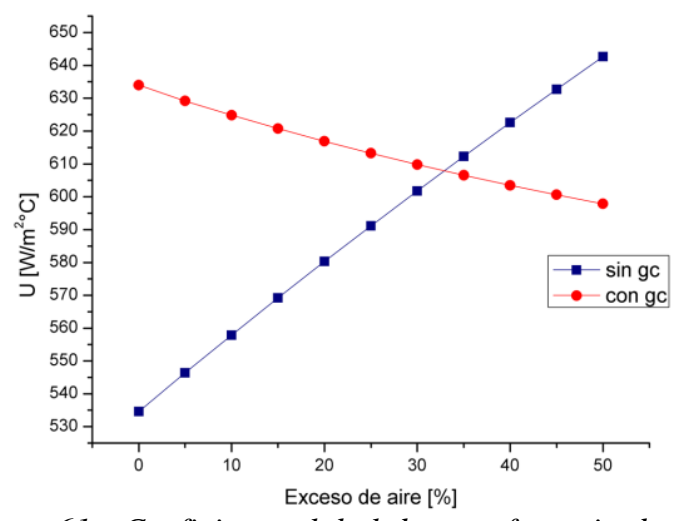

Figura 61.- Coeficiente global de transferencia de calor del GV 80CB con y sin gases provenientes de la MTG C65 con $G N$

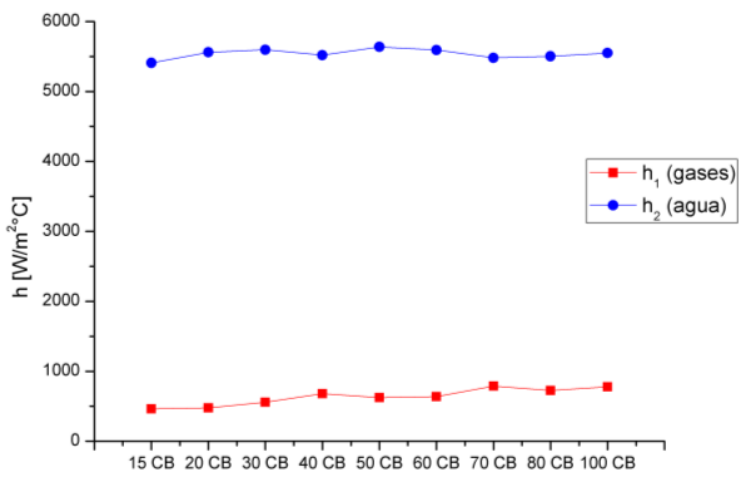

Figura 58.- Coeficientes convectivos $h_{1}$ y $h_{2}$ para cada $G V$ de estudio con los gases provenientes de la MTG C650 con GN. 


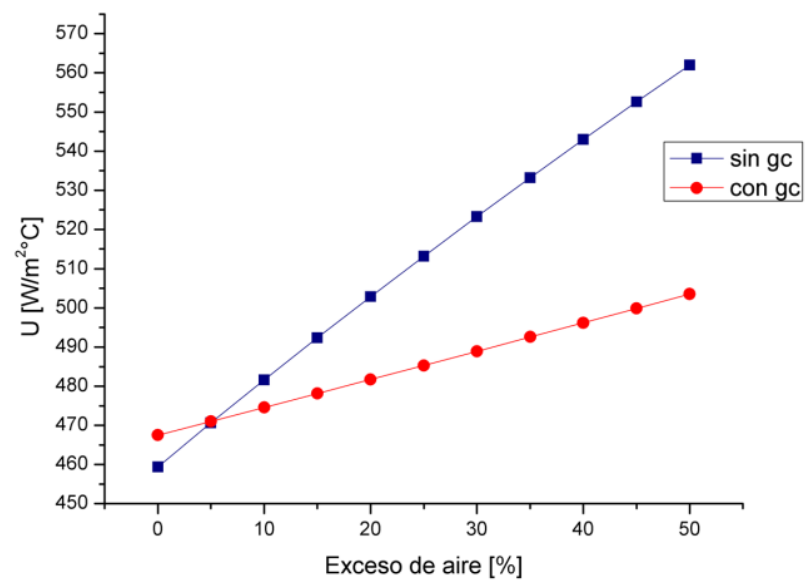

Figura 63.- Coeficiente global de transferencia de calor del GV 80CB con y sin gases provenientes de la MTG C30 con $\mathrm{H}_{2}$

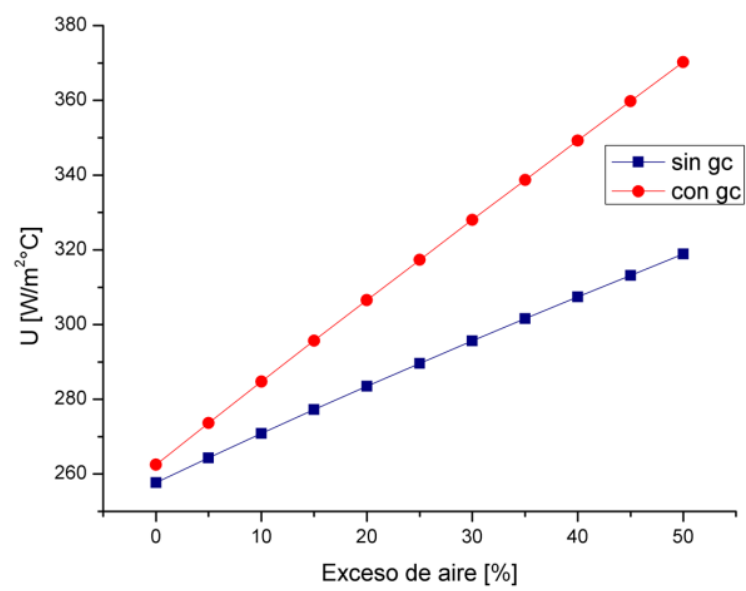

Figura 64.- Coeficiente global de transferencia de calor del GV 80CB con y sin gases provenientes de la MTG C65 con $\mathrm{H}_{2}$

De manera análoga se pueden replicar los cálculos para toda la gama de GV's mostrados en la Tabla 8. A modo de ejemplo, en las Figura 65 a 68 se muestran los resultados obtenidos para todos los GV's bajo estudio a las que se les inyecta los gases provenientes de las MTG's tanto para el coeficiente convectivo $h_{1}$ y para $U$ usando únicamente GN. Como es de esperarse la diferencia en los coeficientes de transferencia de calor $\left(h_{1}\right.$ y $U$ ) son muy parecidos, ya que $h_{1}$ es quien aporta la mayor resistencia en la obtención de U y por ende tienen el mismo comportamiento. Para la MTG C30 se tiene un aumento promedio en el coeficiente convectivo y global de transferencia de calor del $17 \%$ y para la MTG C65 del 20\%.

En cuanto a la caída de presión, al emplear los gases efluentes de la MTG se incrementa el volumen de gas que entra a el GV y más el aire de alimentación adicional, van a provocar un incremento mayor en la caída de presión respecto al sistema convencional, sin embargo este incremento se mantiene casi constante en función del exceso de aire como se muestra en las Figura 69 y 70 , siendo estas del orden de los 80 a 95 mbar.

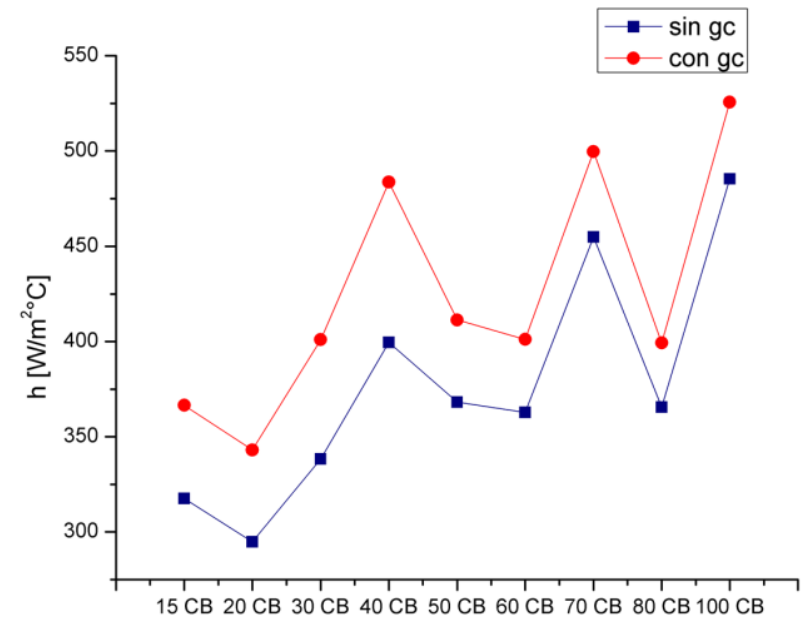

Figura 65.- Coeficiente convectivo para la gama de GV's usando los gases provenientes de la MTG C30 con GN.

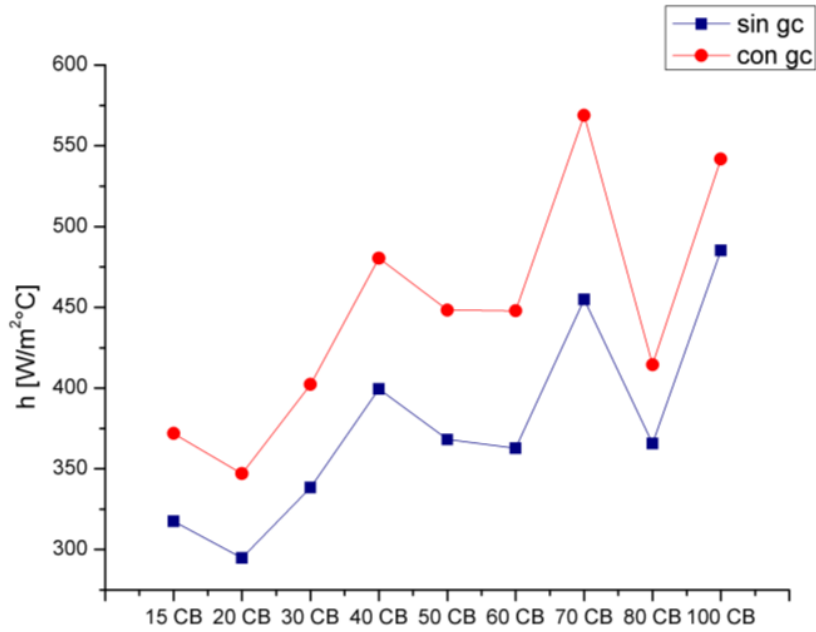

Figura 66.- Coeficiente convectivo para la gama de GV's usando los gases provenientes de la MTG C65 con GN. 


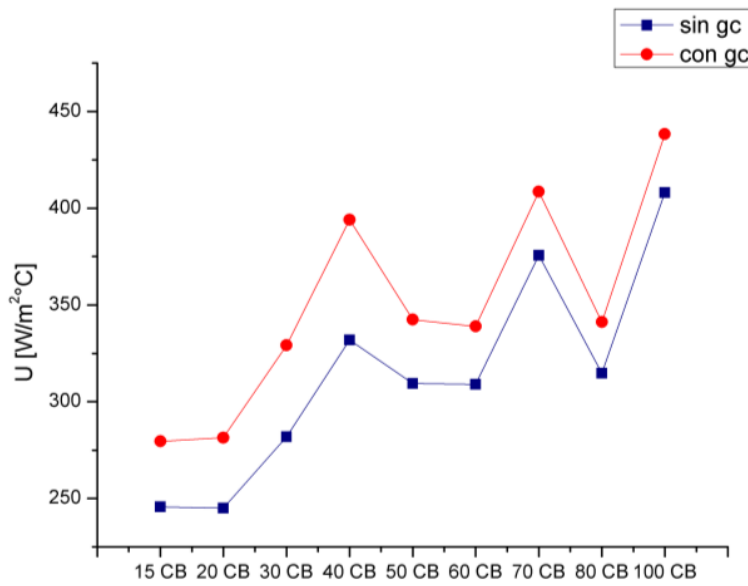

Figura 67.- Coeficiente global de transferencia de calor para la gama de GV's usando los gases provenientes de la $M T G C 30$ con $G N$.

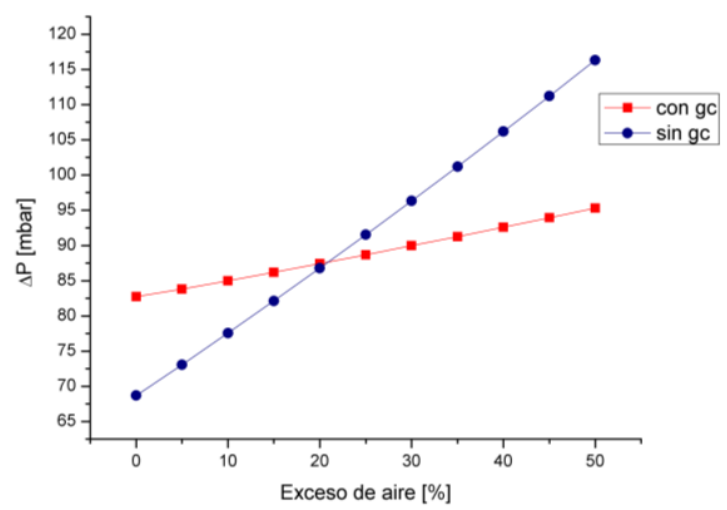

Figura 69.- Caída de presión en el GV 80CB empleando los gases efluentes de la MTG C30.

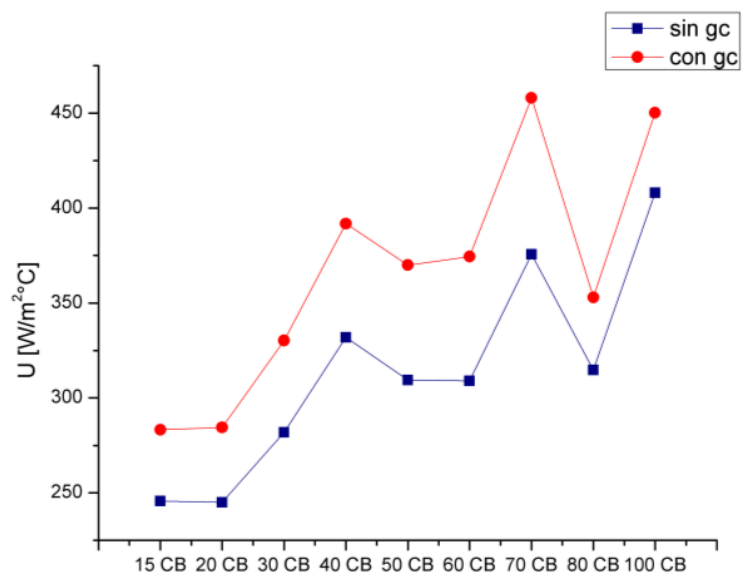

Figura 68.- Coeficiente global de transferencia de calor para la gama de GV's usando los gases provenientes de la MTG C65 con GN

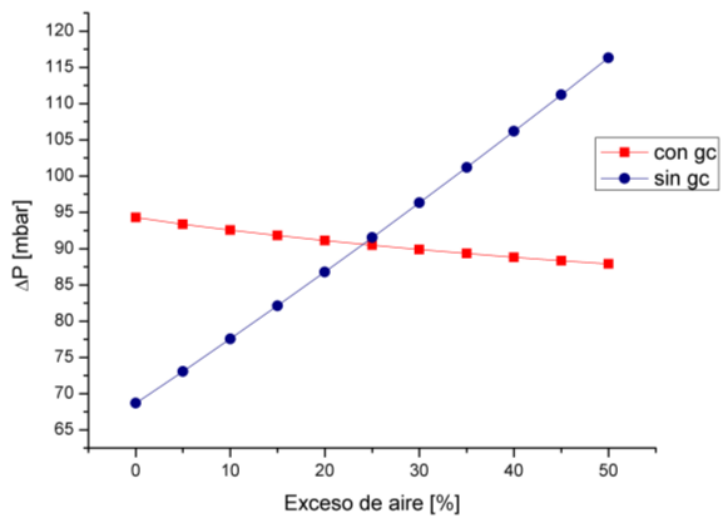

Figura 70.- Caída de presión en el GV 80CB empleando los gases efluentes de la MTG C65.

Conocida la caída de presión, en la Tabla 16 se muestran las potencias mínimas requeridas para el soplador cuando se acoplan los gases efluentes de cada MTG. De acuerdo con la ficha técnica de las calderas, en este caso específico de la $80 \mathrm{CB}$ la potencia del soplador es de $1491.399 \mathrm{~W}$, mayor a la obtenida para cada MTG. Por lo tanto, es suficiente para poder hacer fluir los gases en el GV.

Tabla 16.- Potencia del soplador mínima requerida en el GV.

\begin{tabular}{|c|c|c|}
\hline \multirow{2}{*}{$\begin{array}{c}\text { Exceso de } \\
\text { aire }[\%]\end{array}$} & \multicolumn{2}{|c|}{ Wp [W] } \\
\cline { 2 - 3 } & $\mathbf{C 3 0}$ & $\mathbf{C 6 5}$ \\
\hline $\mathbf{0}$ & 478.63 & 588.81 \\
\hline $\mathbf{5}$ & 491.80 & 583.55 \\
\hline $\mathbf{1 0}$ & 505.62 & 579.08 \\
\hline $\mathbf{1 5}$ & 519.83 & 575.04 \\
\hline $\mathbf{2 0}$ & 534.42 & 571.37 \\
\hline $\mathbf{2 5}$ & 549.38 & 568.06 \\
\hline $\mathbf{3 0}$ & 564.73 & 565.07 \\
\hline $\mathbf{3 5}$ & 580.45 & 562.38 \\
\hline $\mathbf{4 0}$ & 596.55 & 559.97 \\
\hline $\mathbf{4 5}$ & 613.02 & 557.82 \\
\hline $\mathbf{5 0}$ & 629.87 & 555.91 \\
\hline
\end{tabular}


La eficiencia global del sistema para estos casos particulares (C30-GV 80CB y C65- GV 80 CB) se muestran en la Tabla 17. Comparados con la eficiencia global de forma convencional se tenie un incremento del $5.33 \%$ empleando la MTG C30 y del $9.06 \%$ con la C65.

Tabla 17.- Eficiencias globales del sistema convencional vs MCHP

\begin{tabular}{|l|c|c|c|c|}
\hline & \multicolumn{2}{|c|}{ Convencional } & \multicolumn{2}{c|}{ MCHP } \\
\hline & $\mathbf{C 3 0}$ & $\mathbf{C 6 5}$ & $\mathbf{C 3 0}$ & $\mathbf{C 6 5}$ \\
\hline Potencia total útil $[\mathrm{kW}]$ & 807.21 & 831.35 & 807.21 & 831.35 \\
\hline Potencia total suministrada al sistema $[\mathbf{k W}]$ & 1012.57 & 1076.59 & 949.15 & 963.52 \\
\hline Eficiencia global [\%] & $\mathbf{7 9 . 7 2}$ & $\mathbf{7 7 . 2 2}$ & $\mathbf{8 5 . 0 5}$ & $\mathbf{8 6 . 2 8}$ \\
\hline
\end{tabular}

Estos resultados fueron para cuando $\alpha<0$, de la misma manera se pueden hacer los cálculos para una combinación de MTG-GV con $\alpha>0$. A modo de ejemplo se muestran en las Tabla 18 y 19 los resultados obtenidos empleando el GV 20CB con la MTG C30. En las Tabla 20 y 21 se muestran los resultados obtenido empleando el mismo GV pero ahora acoplando la MTG C65.

Tabla 18.- Resultados MTG C30 a 2250msnm y $T=25^{\circ} \mathrm{C}$

\begin{tabular}{|l|c|}
\hline \multicolumn{2}{|c|}{ Resultados MTG C30 } \\
\hline Potencia [kW] & 22.37 \\
\hline Consumo de combustible [kW] & 78.37 \\
\hline Qdisp [kW] & 63.41 \\
\hline Exceso de aire x [\%] & 285.63 \\
\hline T exhaust gasses [ $\left.{ }^{\circ} \mathbf{C}\right]$ & 285.42 \\
\hline mgc [kg/s] & 0.2341 \\
\hline $\boldsymbol{\pi}$ & 3.2 \\
\hline $\mathbf{E}$ gc usados $\boldsymbol{\alpha}>\mathbf{0}[\mathbf{k g} / \mathbf{s}]$ & 0.1131 \\
\hline Flujo desviado cuando $\boldsymbol{\alpha}>\mathbf{0}[\mathbf{k g} / \mathbf{s}]$ & $\mathbf{0 . 1 2 1 0}$ \\
\hline $\mathbf{Q}_{\text {disp } \text { usado } \boldsymbol{\alpha}>\mathbf{0}[\mathbf{k W}]}$ & 30.79 \\
\hline $\mathbf{Q}_{\text {faltante }} \boldsymbol{\alpha}>\mathbf{0}[\mathbf{k W}$ & 32.62 \\
\hline
\end{tabular}

Tabla 20.- Resultados MTG C65 a 2250msnm y $T=25^{\circ} \mathrm{C}$

\begin{tabular}{|l|c|}
\hline Resultados MTG C65 & \\
\hline Potencia [kW] & 46.50 \\
\hline Consumo de combustible [kW] & 142.39 \\
\hline Qdisp [kW] & 113.07 \\
\hline Exceso de aire x [\%] & 242.75 \\
\hline T exhaust gasses [ $\left.{ }^{\circ} \mathbf{C}\right]$ & 318.74 \\
\hline mgc [kg/s] & 0.3701 \\
\hline $\boldsymbol{\pi}$ & 3.7 \\
\hline $\boldsymbol{\varepsilon}$ gc usados $\boldsymbol{\alpha}>\mathbf{0}[\mathbf{k g} / \mathbf{s}]$ & 0.1164 \\
\hline Flujo desviado cuando $\boldsymbol{\alpha}>\mathbf{0}$ [kg/s] & 0.2537 \\
\hline Qdisp usado $\boldsymbol{\alpha}>\mathbf{0}$ [kW] & 35.75 \\
\hline Qfaltante $\boldsymbol{\alpha}>\mathbf{0}$ [kW] & 77.32048 \\
\hline
\end{tabular}

Tabla 19.- Resultados del GV 20CB usando los gases de combustión de la MTG C30

\begin{tabular}{|c|c|}
\hline \multicolumn{2}{|l|}{ Resultados GV } \\
\hline & $\alpha>0 \times 10 \%$ \\
\hline Potencia [kW] & 196.36 \\
\hline Qsum $[k W]$ & 238.08 \\
\hline$\dot{\mathbf{m}}_{\text {comb }}[\mathrm{kg} / \mathrm{s}]$ & 0.00488 \\
\hline $\mathbf{Q}_{\text {sum2 }}[\mathbf{k W}]$ & 207.29 \\
\hline$\dot{\mathbf{m}}_{\mathrm{comb} 2}[\mathrm{~kg} / \mathrm{s}]$ & 0.00425 \\
\hline hc a $10 \%$ de exceso de aire $\left[\mathrm{W} / \mathrm{m}^{2} \mathrm{~K}\right]$ & 399.47 \\
\hline$h_{\text {TG-C }}$ a $10 \%$ de exceso de aire $\left[\mathrm{W} / \mathrm{m}^{2} \mathrm{~K}\right]$ & 469.36 \\
\hline$\%$ incremento en el coef. Conv. Transf. & 17.49 \\
\hline $\mathrm{U}_{G V}$ a $10 \%$ de exceso de aire $\left[\mathrm{W} / \mathrm{m}^{2} \mathrm{~K}\right]$ & 359.35 \\
\hline $\mathrm{UTG}_{\mathrm{TG}-\mathrm{V}}$ a $10 \%$ de exceso de aire $\left[\mathrm{W} / \mathrm{m}^{2} \mathrm{~K}\right]$ & 416.97 \\
\hline \% incremento en el coef. Global Transf. & 16.04 \\
\hline
\end{tabular}

Tabla 21.- Resultados del GV 20CB usando los gases de combustión de la MTG C65

\begin{tabular}{|l|c|}
\hline \multicolumn{2}{|c|}{ Resultados GV } \\
\hline & $\boldsymbol{\alpha}>\mathbf{0}$ x=10\% \\
\hline Potencia [kW] & 196.36 \\
\hline Qsum [kW] & 238.08 \\
\hline mcomb [kg/s] & 0.00488 \\
\hline Qsum2 [kW] & 202.34 \\
\hline mcomb2 [kg/s] & 0.00415 \\
\hline hC a 0\% de exceso de aire [W/m2K] & 399.47 \\
\hline hTG-C a 0\% de exceso de aire [W/m2K] & 475.79 \\
\hline \% incremento en el coef. Conv. Transf. & $\mathbf{1 9 . 1 1}$ \\
\hline UC a 0\% de exceso de aire [W/m2K] & 359.35 \\
\hline UTG-C a 0\% de exceso de aire [W/m2K] & 422.21 \\
\hline \% incremento en el coef. Global Transf. & $\mathbf{1 7 . 4 9}$ \\
\hline
\end{tabular}

Conocidos los datos anteriores, se pueden obtener las eficiencias globales del sistema de MCHP. En la Tabla 22 se muestran las eficiencias globales operando de forma convencional y con el sistema de MCHP para cada una de las MTG's acopladas con el GV 20CB. Obteniendo un incremento empleando la MTG C30 y C65 del 7.45\% y 6.62\% respectivamente. La MTG C65 tiene el menor 
incremento debido a que se tiene que desviar más flujo de gases de combustión al medio ambiente. Por lo tanto, para este caso particular lo recomendado es acoplar la MTG C30 al GV 20CB, ya que se obtiene la mayor eficiencia y se aprovecha la mayor cantidad de energía disponible por los gases efluentes de esta.

Tabla 22.- Resultados de las eficiencias del sistema convencional vs MCHP usando el GV 20 CB.

\begin{tabular}{|l|c|c|}
\hline $\boldsymbol{\alpha}>\mathbf{0}$ & $\mathbf{C 3 0}$ & $\mathbf{C 6 5}$ \\
\hline Eficiencia de la TG & 28.54 & 32.66 \\
\hline Eficiencia de la caldera & 82.47 & 82.47 \\
\hline Eficiencia del sistema Convencional [\%] & $\mathbf{6 9 . 1 2}$ & $\mathbf{6 3 . 8 3}$ \\
\hline Potencia total útil [kW] & 218.72 & 242.86 \\
\hline Potencia total suministrada al sistema [kW] & 285.66 & 344.73 \\
\hline Eficiencia del sistema MCHP [\%] & $\mathbf{7 6 . 5 7}$ & $\mathbf{7 0 . 4 5}$ \\
\hline
\end{tabular}

\subsection{Aplicación de la metodología de cogeneración eficiente a las Micro-Turbinas de Gas}

En este trabajo la evaluación del sistema de MCHP se hace para la CDMX considerando una altura de $2250 \mathrm{msnm}$ y una temperatura media anual de $25^{\circ} \mathrm{C}$. La evaluación se hace para los 3 combustibles y las 2 turbinas bajo estudio que operan 8,390 horas al año con una disponibilidad del $95.77 \%$, ya considerando el mantenimiento mayor (Overhaul).

Para el caso del hidrógeno, se consideró como si este fuera proveniente de un combustible fósil, de lo contrario, si éste fuera por una fuente renovable el \%ELC es de $100 \%$.

En las Tabla 23 y 24 se muestran los resultados obtenidos al aplicar la metodología de cogeneración eficiente para ambas MTG's. Se observa que empleando GN se obtiene un mayor \%ELC. Para el caso del $\mathrm{H}_{2}$, este rebasa el $100 \%$ de ELC, esto debido al alto calor disponible que se tiene y que consume un flujo de combustible mucho menor que los otros dos combustibles.

Para los certificados de energía limpia (CEL), que son los bonos que otorga la LIE en función de la energía libre de combustible, y se otorga por cada MWh/año que se genera de forma limpia, se determinó que para ambas turbinas se obtiene mayor número de CEL's empleando GN (sin considerar al hidrógeno).

Tabla 23.- Resultados obtenidos de la metodología de cogeneración eficiente para la MTG C30 con 3 combustibles diferentes

\begin{tabular}{|l|c|c|c|}
\hline \multicolumn{1}{|c|}{ CAPSTONE C30 } & GN & GLP & H $_{2}$ \\
\hline Potencia ISO [kW] & \multicolumn{3}{c|}{30} \\
\hline Potencia in situ [kW] & 22.37 & 22.41 & 23.52 \\
\hline Consumo de combustible [kW] & 78.37 & 78.51 & 91.51 \\
\hline No de horas de operación anual [h/año] & $8,390.00$ & $8,390.00$ & $8,390.00$ \\
\hline E [kWh/año] & $187,649.94$ & $187,988.30$ & $197,368.85$ \\
\hline F [kWh/año] & $657,502.81$ & $658,688.37$ & $767,734.45$ \\
\hline HSG [kW] & 52.72 & 51.06 & 59.96 \\
\hline H [kWh/año] & $442,327.58$ & $428,431.58$ & $503,104.33$ \\
\hline Re [\%] & $28.54 \%$ & $28.54 \%$ & $25.71 \%$ \\
\hline Rh [\%] & $67.27 \%$ & $65.04 \%$ & $65.53 \%$ \\
\hline RefE [\%] & $44.00 \%$ & $44.00 \%$ & $44.00 \%$ \\
\hline RefH [\%] & $82.00 \%$ & $82.00 \%$ & $82.00 \%$ \\
\hline fp [\%] & $94.00 \%$ & $94.00 \%$ & $94.00 \%$ \\
\hline
\end{tabular}




\begin{tabular}{|l|c|c|c|}
\hline \multicolumn{1}{|c|}{ CAPSTONE C30 } & GN & GLP & H $_{\mathbf{2}}$ \\
\hline RefE' [\%] & $41.36 \%$ & $41.36 \%$ & $41.36 \%$ \\
\hline Fh [kWh/año] & $539,423.87$ & $522,477.54$ & $613,541.87$ \\
\hline Fe [kWh/año] & $118,078.94$ & $136,210.83$ & $154,192.58$ \\
\hline EE [\%] & $158.92 \%$ & $138.01 \%$ & $128.00 \%$ \\
\hline Econv [kWh/año] & $51,954.73$ & $59,932.76$ & $67,844.74$ \\
\hline EP [kWh/año] & $993,122.96$ & $976,994.70$ & $1,090,739.3$ \\
\hline AEP [kWh/año] & $335,620.14$ & $318,306.33$ & $323,004.83$ \\
\hline APEP [\%] & $33.79 \%$ & $32.58 \%$ & $29.61 \%$ \\
\hline AREL [\%] & $284.23 \%$ & $233.69 \%$ & $209.48 \%$ \\
\hline ELC [kWh/año] & $147,672.86$ & $140,054.79$ & $142,122.13$ \\
\hline \%ELC & $\mathbf{7 8 . 7 0 \%}$ & $\mathbf{7 4 . 5 0 \%}$ & $\mathbf{7 2 . 0 1 \%}$ \\
\hline CEL [MWh/año] & $\mathbf{1 4 7 . 6 7}$ & $\mathbf{1 4 0 . 0 5}$ & $\mathbf{1 4 2 . 1 2}$ \\
\hline
\end{tabular}

Tabla 24.- Resultados obtenidos de la metodología de cogeneración eficiente para la MTG C65 con 3 combustibles diferentes

\begin{tabular}{|l|c|c|c|}
\hline \multicolumn{1}{|c|}{ CAPSTONE C65 } & GN & GLP & H$_{2}$ \\
\hline Potencia ISO [kW] & \multicolumn{3}{c|}{65} \\
\hline Potencia in situ [kW] & 46.50 & 46.58 & 48.98 \\
\hline Consumo de combustible [kW] & 142.39 & 142.63 & 183.12 \\
\hline No de horas de operación anual [h/año] & $8,390.00$ & $8,390.00$ & $8,390.00$ \\
\hline E [kWh/año] & $390,158.49$ & $390,813.00$ & $410,940.32$ \\
\hline F [kWh/año] & $1,194,643.87$ & $1,196,647.93$ & $1,536,357.68$ \\
\hline HSG [kW] & 96.23 & 93.21 & 118.03 \\
\hline H [kWh/año] & $807,374.22$ & $782,015.18$ & $990,247.10$ \\
\hline Re [\%] & $32.66 \%$ & $32.66 \%$ & $26.75 \%$ \\
\hline Rh [\%] & $67.58 \%$ & $65.35 \%$ & $64.45 \%$ \\
\hline RefE [\%] & $44.00 \%$ & $44.00 \%$ & $44.00 \%$ \\
\hline RefH [\%] & $82.00 \%$ & $82.00 \%$ & $82.00 \%$ \\
\hline fp [\%] & $94.00 \%$ & $94.00 \%$ & $94.00 \%$ \\
\hline RefE' [\%] & $41.36 \%$ & $41.36 \%$ & $41.36 \%$ \\
\hline Fh [kWh/año] & $984,602.71$ & $953,677.05$ & $1,207,618.41$ \\
\hline Fe [kWh/año] & $210,041.16$ & $242,970.88$ & $328,739.27$ \\
\hline EE [\%] & $185.75 \%$ & $160.85 \%$ & $125.00 \%$ \\
\hline Econv [kWh/año] & $92,418.11$ & $106,907.19$ & $144,645.28$ \\
\hline EP [kWh/año] & $1,927,925.94$ & $1,898,582.75$ & $2,201,187.9$ \\
\hline AEP [kWh/año] & $733,282.08$ & $701,934.82$ & $664,830.18$ \\
\hline APEP [\%] & $38.03 \%$ & $36.97 \%$ & $30.20 \%$ \\
\hline AREL [\%] & $349.11 \%$ & $288.90 \%$ & $202.24 \%$ \\
\hline ELC [kWh/año] & $322,644.11$ & $308,851.32$ & $292,525.28$ \\
\hline \%ELC & $\mathbf{8 2 . 7 0 \%}$ & $\mathbf{7 9 . 0 3 \%}$ & $\mathbf{7 1 . 1 8 \%}$ \\
\hline CEL [MWh/año] & $\mathbf{3 2 2 . 6 4}$ & $\mathbf{3 0 8 . 8 5}$ & $\mathbf{2 9 2 . 5 3}$ \\
\hline
\end{tabular}

\subsection{Emisiones de $\mathrm{CO}_{2}$}

A partir de la metodología mostrada en el capítulo 6, se obtienen las toneladas de $\mathrm{CO}_{2}$ emitidas por cada MWh/año generado considerando que operan 8,390 horas al año. En las Tabla 25 y 26 se muestran los resultados obtenidos para la MTG C30 y C65 respectivamente. En la Tabla 27 se muestran las emisiones evitadas con el sistema de MCHP, siendo estas en promedio del $8 \%$ (dependiendo del combustible usado), con respecto a los sistemas por separado. Además, se tiene aproximadamente un $10 \%$ de ahorro en el consumo de combustible. 
Tabla 25.- Toneladas de $\mathrm{CO}_{2}$ por equipo y combustible emitidas (MTG C30)

\begin{tabular}{|l|c|c|c|c|c|c|}
\hline \multicolumn{1}{|c|}{ Equipo } & \multicolumn{2}{c|}{ MTG } & \multicolumn{2}{c|}{ GV } & \multicolumn{2}{c|}{ MCHP } \\
\hline Combustible & GN & GLP & GN & GLP & GN & GLP \\
\hline m $\mathbf{C O}_{2}[\mathbf{k g} / \mathbf{s}]$ & 0.0097 & 0.0112 & 0.0530 & 0.0614 & 0.0591 & 0.0686 \\
\hline $\mathbf{t C O} / \mathbf{a n ̃ o}$ & 291.98 & 338.62 & 1600.96 & 1855.61 & 1783.72 & 2071.61 \\
\hline $\mathbf{t C O} / \mathbf{M W h} / \mathbf{a n ̃ o}$ & 1.5560 & 1.8013 & 0.2431 & 0.2818 & 0.2634 & 0.3059 \\
\hline
\end{tabular}

Tabla 26.- Toneladas de $\mathrm{CO}_{2}$ por equipo y combustible emitidas (MTG C65)

\begin{tabular}{|l|c|c|c|c|c|c|}
\hline \multicolumn{1}{|c|}{ Equipo } & \multicolumn{2}{c|}{ MTG } & \multicolumn{2}{c|}{ GV } & \multicolumn{2}{c|}{ MCHP } \\
\hline Combustible & GN & GLP & GN & GLP & GN & GLP \\
\hline m $\mathbf{C O}_{2}[\mathbf{k g} / \mathbf{s}]$ & 0.0172 & 0.0199 & 0.0530 & 0.0614 & 0.0637 & 0.0741 \\
\hline tCO $/$ /año & 518.32 & 601.12 & 1600.96 & 1855.61 & 1924.54 & 2238.10 \\
\hline tCO$_{2} / \mathbf{M W h} / \mathbf{a n ̃ o}$ & 1.3285 & 1.5381 & 0.2431 & 0.2818 & 0.2759 & 0.3208 \\
\hline
\end{tabular}

Tabla 27.- Emisiones evitadas con el sistema de MCHP

\begin{tabular}{|l|c|c|c|c|}
\hline & \multicolumn{1}{|c|}{ tCO$/ \mathbf{a n ̃ o ~ e v i t a d a s ~}$} & \multicolumn{1}{|c|}{ tCO $/$ /MWh/año evitadas } \\
\hline Combustible & GN & GLP & GN & GLP \\
\hline C30 & 109.22 & 122.62 & 0.0161 & 0.0181 \\
\hline C65 & 194.75 & 218.63 & 0.0279 & 0.0313 \\
\hline
\end{tabular}

\subsection{Interface de programa}

Usando la metodología de cálculo presentada en este trabajo, se realizó un programa en Excel acoplado al software Steam. En este programa se puede elegir la turbina de estudio, el combustible a emplear, la altura sobre el nivel del mar, la temperatura ambiente del sitio; para obtener la potencia real que estregará el equipo. También se puede elegir el GV con la que se desea acoplar los gases provenientes de la MTG. Mostrando los resultados para la MTG y el GV seleccionado. También se muestran las emisiones de $\mathrm{CO}_{2}$ evitadas comparadas con los sistemas convencionales por separado.

Cuando se elige como combustible el hidrógeno, los resultados se muestran en una tabla y gráficos independientes, como se muestra en la Figura 71.

La metodología de cogeneración eficiente va inmersa en la misma interface, en la Figura 72 se muestra un zoom para poder observar los parámetros que se obtienen para calcular la energía libre de combustible con cada una de las turbinas, combustibles y condiciones atmosféricas que se elijan. 


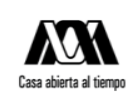
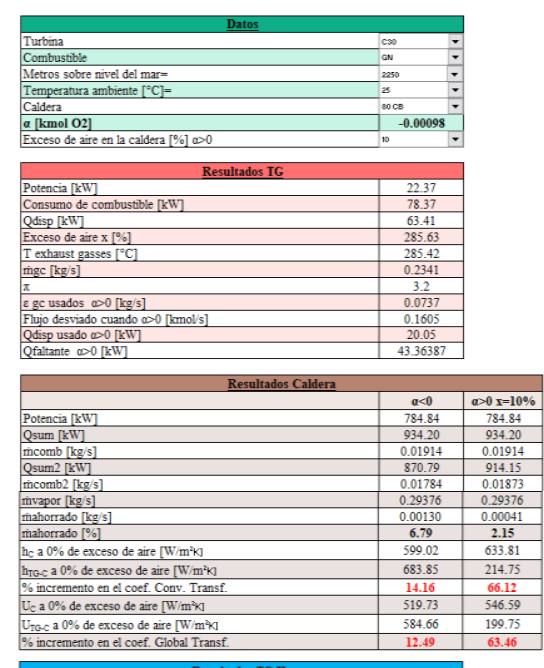

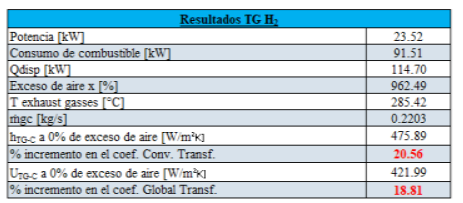
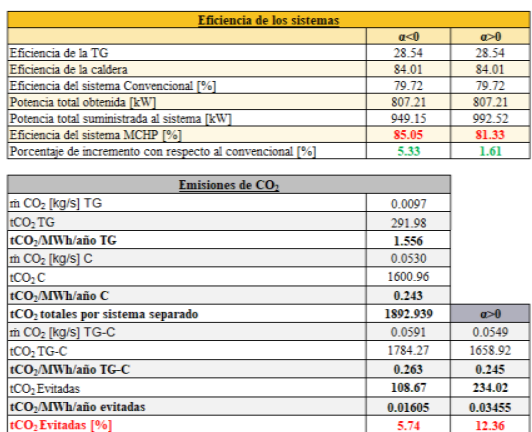

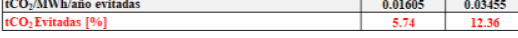

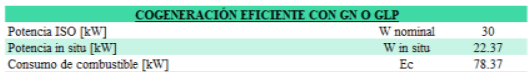

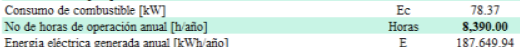

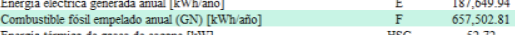
Energit témica de gases de escape [kT] Rendiniento eléctrico del sistema [\%]

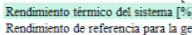

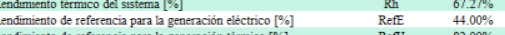

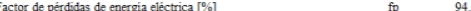

Rendimiento de referenencia para la generación eléctrica a tensiön del si

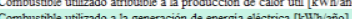
Eficiencia artibuible a la generacion eléctrica [\%]

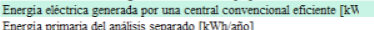

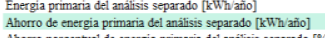
Aporte relativo de energia Iimpia de una central dectrica CHP con rest Energiga electrica thbre de combustible (de caracter renovable) [kWha

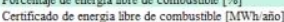

POtencia ISO [KW] COGENERACTON EFICIENIE CONH, Potencia Iso $[\mathrm{kWW}]$

Consumo de combustible $[k \mathrm{~kW}]$
No de horas de operacion anual [hanio]

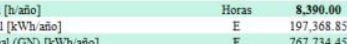
Energia termica de gases de escape [kW] Energia termica neta anual [kWh ailo] Rendimiento elictrico del sistema [\%?
Rendimiento témico del sistema $[\%]$

\begin{tabular}{ll} 
Reff & $4.00 \%$ \\
\hline
\end{tabular}

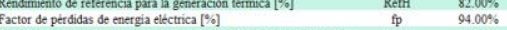

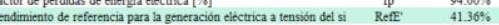

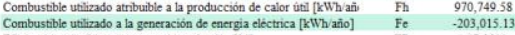
Ficencia atrbuable a la generación electrica [\%]

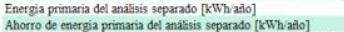
Ahorro de energia primaria del anaisisis separado [kWh ahio]
Ahoorto porcentual de energia primaria del anásisis separado [\%

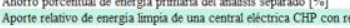
Energia electrica ibre de combustibie (de caracter renovable) [KW What

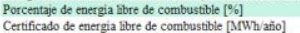

Universidad Autónoma Metropolitana-Iztapalapa Posgrado de Energia y Medio Ambiente Ing. Adriana Santamaria Padilla
Dr. Hermando Romero Paredes Rubio
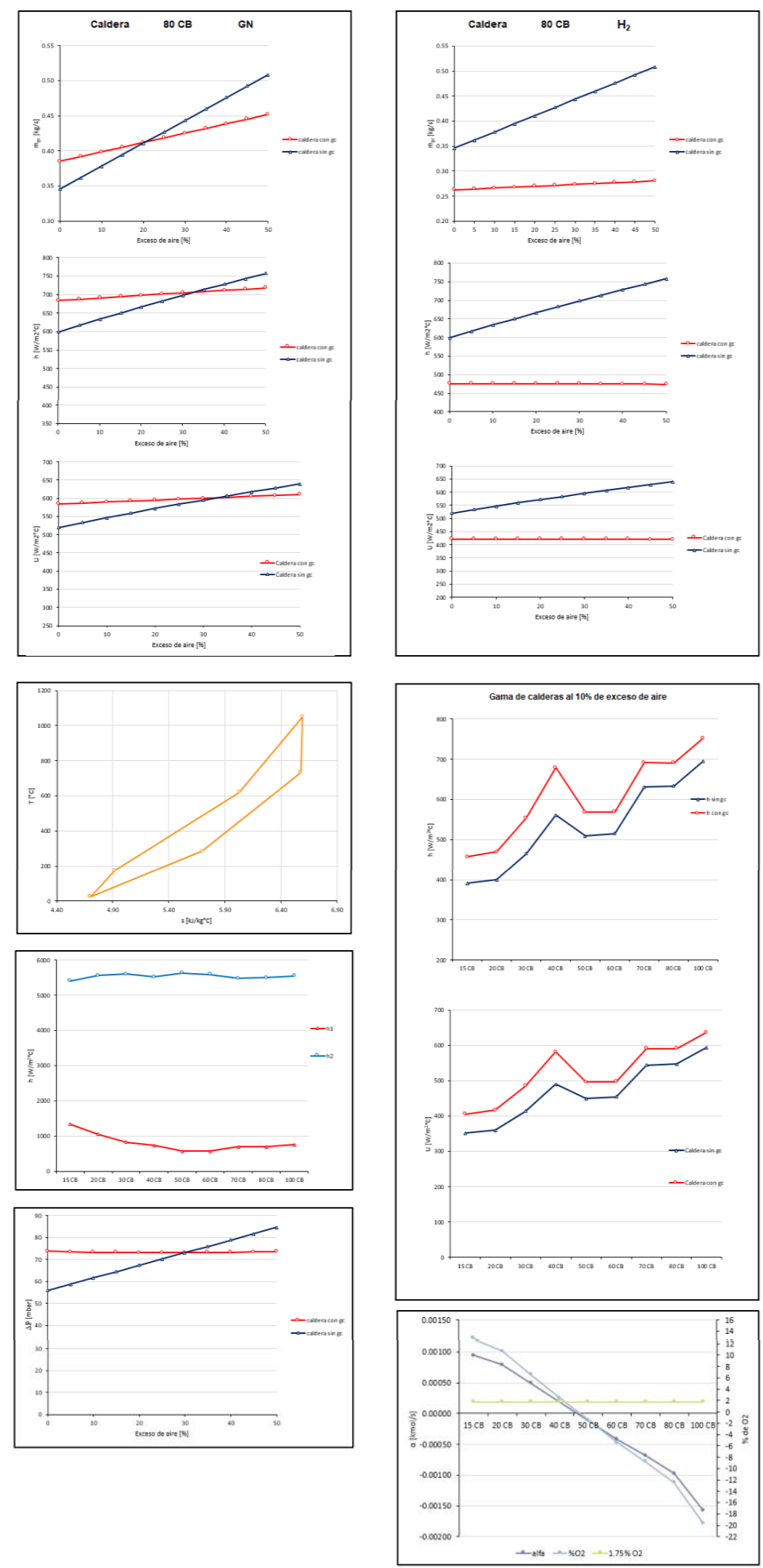

Figura 71.- Interface del programa realizado en Excel 


\begin{tabular}{|c|c|c|}
\hline \multicolumn{3}{|c|}{ COGENERACIÓN EFICIENTE CON GN O GLP } \\
\hline Potencia ISO [kW] & W nominal & 30 \\
\hline Potencia in situ $[\mathrm{kW}]$ & $\mathrm{W}$ in situ & 22.37 \\
\hline Consumo de combustible $[\mathrm{kW}]$ & Ec & 78.37 \\
\hline No de horas de operación anual [h/año] & Horas & $8,390.00$ \\
\hline Energia eléctrica generada anual [kWh/año] & E & $187,649.94$ \\
\hline Combustible fósil empelado anual (GN) [kWh/año] & F & $657,502.81$ \\
\hline Energia térmica de gases de escape $[\mathrm{kW}]$ & HSG & 52.72 \\
\hline Energia térmica neta anual [kWh/año] & $\mathrm{H}$ & $442,327.58$ \\
\hline Rendimiento eléctrico del sistema [\%] & $\operatorname{Re}$ & $28.54 \%$ \\
\hline Rendimiento térmico del sistema [\%] & $\mathrm{Rh}$ & $67.27 \%$ \\
\hline Rendimiento de referencia para la generación eléctrico [\%] & RefE & $44.00 \%$ \\
\hline Rendimiento de referencia para la generación térmica [\%] & RefH & $82.00 \%$ \\
\hline Factor de pérdidas de energia eléctrica [\%] & $\mathrm{fp}$ & $94.00 \%$ \\
\hline Rendimiento de referencia para la generación eléctrica a tensión del sistem & RefE' & $41.36 \%$ \\
\hline Combustible utilizado atribuible a la producción de calor útil [kWh/año] & $\mathrm{Fh}$ & $539,423.87$ \\
\hline Combustible utilizado a la generación de energia eléctrica [kWh/año] & $\mathrm{Fe}$ & $118,078.94$ \\
\hline Eficiencia atribuible a la generación eléctrica [\%] & $\mathrm{EE}$ & $158.92 \%$ \\
\hline Energia eléctrica generada por una central convencional eficiente $[\mathrm{kWh} /$ añc & Econv & $51,954.73$ \\
\hline Energia primaria del análisis separado [kWh/año] & EP & $993,122.96$ \\
\hline Ahorro de energia primaria del análisis separado [kWh/año] & AEP & $335,620.14$ \\
\hline Ahorro porcentual de energia primaria del análisis separado [\%] & APEP & $33.79 \%$ \\
\hline Aporte relativo de energia limpia de una central eléctrica CHP con relación & AREL & $284.23 \%$ \\
\hline Energia eléctrica libre de combustible (de carácter renovable) [kWh/año] & ELC & $147,672.86$ \\
\hline Porcentaje de energia libre de combustible [\%] & $\%$ ELC & $78.70 \%$ \\
\hline Certificado de energia libre de combustible [MWh/año] & CEL & 147.67 \\
\hline
\end{tabular}

Figura 72.- Interface de cálculo para la metodología de cogeneración eficiente. 


\section{Capítulo 8. Conclusiones}

En este trabajo se encontró que las condiciones de operación de la MTG Capstone C30 es con una $\pi=3.2$ y TET $=1050^{\circ} \mathrm{C}$. A partir del coeficiente de derrateo se observa que se tiene un decremento en la potencia del $5.5 \%$ por cada $500 \mathrm{msnm}$ y del $3.9 \%$ por cada $5^{\circ} \mathrm{C}$ que aumenta la temperatura promedio del medio ambiente. Para la MTG Capstone C65 se encontró que opera con una $\pi=3.7 \mathrm{y}$ TET $=1200{ }^{\circ} \mathrm{C}$. A partir del coeficiente de derrateo se observa que se tiene un decremento en la potencia del $6.2 \%$ por cada $500 \mathrm{msnm}$ y del $3.8 \%$ por cada $5^{\circ} \mathrm{C}$ que aumenta la temperatura promedio del medio ambiente.

Para empleo de los gases de combustión de las MTG's en un GV, en principio ambas puedes ser utilizadas, pero para obtener la mayor eficiencia en el sitemas de MCHP se buscaría acoplar las MTG's cuando se emplee la mayor cantidad de energía disponible. Es decir, para cuando $\alpha$ es menor a cero. Por lo tanto, se encontró que las MTG's pueden cubrir las necesidades de los GV's para cuando $\alpha<0$ y se aprovecha toda de la siguiente manera:

- Gas Natural

- C30 cubre un intervalo de potencias de 50-100 BHP

- C65 cubre un intervalo de potencias de 80-100 BHP

- Gas LP

- C30 cubre un intervalo de potencias de 50-100 BHP

- C65 cubre un intervalo de potencias de 80-100 BHP

- Hidrógeno

- C30 cubre un intervalo de potencias de 70-100 BHP

- C65 para toda la gama de calderas $\alpha>0$

Una vez que se ubica la turbina bajo condiciones de operación real, a una temperatura y presión determinadas, que en este caso son las de la Ciudad de México, se evaluó el efecto que se tiene en el coeficiente convectivo de transferencia de calor del lado de los gases en el GV, con el suministro de los gases de combustión provenientes de la MGT para sustituir una fracción del aire requerido para la combustión y generación de vapor. El ahorro de energía determinado en este trabajo equivale al 10\% del combustible suministrado de forma convencional, a este ahorro hay que sumarle los beneficios que se obtienen con el incremento del coeficiente convectivo de transferencia de calor, que con un bajo exceso de aire (10\%) se puede tener entre $15-20 \%$ de incremento en ese coeficiente dependiendo del combustible usado (GN, GLP e $\mathrm{H}_{2}$ ).

Al tener este incremento en ese coeficiente y demostrar que, si es factible emplear los gases provenientes de la MTG e ingresarlos al hogar de un GV pre-existente, se evita la inversión de una caldera de recuperación de calor (HRSG) o en su caso de un economizador para el GV. Sin embargo, será necesario cambiar el quemador del GV por uno que soporte altas temperaturas $\left(270-320^{\circ} \mathrm{C}\right)$, como por ejemplo el quemador de la marca SAACKE modelo DDZG-GTM, su ficha técnica se muestra en el Anexo C.

Sumado a esto se tiene una reducción sustancial en las emisiones de $\mathrm{CO}_{2}$ generadas por cada MWh/año, siendo este en promedio del 8\% dependiendo del combustible empleado. Adicionalmente se tiene el beneficio de la generación eléctrica in situ que permite garantizar un suministro continuo, sin cortes y con una excelente calidad. Se tiene también el acceso a certificados de energía limpia (CEL) a través de la energía libre de combustible determinada en el último capítulo. Como puede 
observarse entre mayor sea la turbina su eficiencia es mayor y, por consecuencia, se pueden obtener mejores resultados económicos.

Por lo anterior, se demuestra ampliamente la ventaja de emplear un sistema convencional de generación de vapor para la MCHP y se tendría un impacto positivo en la meta nacional de reducción de emisiones al emplearse este tipo de sistemas en todo el país.

Finalmente, de los resultados obtenidos de este trabajo se participó en congresos y se hizo una publicación de difusión los cuales se muestran en el Anexo D. 


\section{Capítulo 9. Recomendaciones y perspectivas}

Con los resultados obtenidos en este trabajo, se hace evidente que haría falta la puesta en práctica de este tipo de sistemas y por ende hacer una instalación de una MTG a un GV pirotubular pre-existente en la industria y hacer el monitoreo de todas las variables para contrastarlas con la metodología planteada en este. Así también se abre una oportunidad de capacitación en el mismo centro donde se haga la instalación y poder replicar el sistema acorde a las necesidades particulares de cada industria. Finalmente se podría realizar un análisis del potencial de MCHP en México, el cual actualmente se desconoce.

El estudio se hizo para 3 combustibles (GN, GLP e $\mathrm{H}_{2}$ ), donde el hidrógeno puede ser proveniente de una fuente fósil o renovable. Cuando el hidrógeno proviene de una fuente renovable, esta puede ser por concentración solar a través de un proceso termoquímico. Se puede aprovechar el recurso solar para el calentamiento del aire después de ser comprimido en el ciclo de la turbina de gas, si con esta energía es suficiente para alcanzar las condiciones de operación no se requerirá de la inyección de hidrógeno, con lo cual se podría trabajar durante el día con concentración solar y por la noche quemando hidrógeno, que ha sido demostrada ampliamente su factibilidad.

Si se llegará a ocupar hidrógeno con oxígeno puro, probablemente se tendría problemas en los alabes de la MTG, ya que se alcanzarían temperaturas muy elevadas poniendo en riesgo la resistencia de los alabes y de ser así se tendrían que fabricar con algún cerámico que soporte las altas temperaturas. 


\section{Referencias}

[1] GIZ México, “Micro y Pequeña Cogeneración y Trigeneración en México,” 2013.

[2] WADE, “Guide to Decentralized Energy Technologies," 2003.

[3] M. Pehnt, M. Cames, C. Fischer, B. Praetorius, L. Schneider, and K. Schumacher, Micro Cogeneration Towards Decentralized Energy Systems. 2006.

[4] H. I. Onovwiona and V. I. Ugursal, "Residential cogeneration systems: review of the current technology," Renew. Sustain. Energy Rev., vol. 10, no. 5, pp. 389-431, Oct. 2006.

[5] IPCC, "Climate Change 2013: The Physical Science Basis. Contribution of Working Group I to the Fifth Assessment Report of the Intergovernmental Panel on Climate Change," p. 33, 2013.

[6] FAO, "Cambio climático," 2017. [Online]. Available: http://www.fao.org/climate-change/es/.

[7] A. Bautista, M. Martínez, and G. Paredes, "Guía de Usuario Registro Nacional de Emisiones (RENE)," Manager, no. Julio, pp. 1-64, 2015.

[8] Cámara de Diputados del H. Congreso de la Unión, "Ley General De Cambio Climático," Dof 13-07-2018, pp. 1-58, 2018.

[9] CONUEE, CRE, and GIZ México, "Estudio sobre Cogeneración en el Sector Industrial en México," Conuee, Com. Nac. Para el Uso Efic. la Energ., pp. 1-156, 2009.

[10] P. A. Pilavachi, "Mini- and micro-gas turbines for combined heat and power," Appl. Therm. Eng., vol. 22, no. 18, pp. 2003-2014, 2002.

[11] T. Giampaolo, Gas Turbine Handbook: Principles and Practice. Fairmont Press, 2009.

[12] F. Draft, "Industrial Applications for Micropower: A Market Assessment," no. November, 1999.

[13] M. Public, U. Commission, R. D. Corporation, and R. D. Corporation, "Assessment of Distributed Generation Technology Applications," vol. 22182, no. February, 2001.

[14] E. P. Agency, C. Protection, P. Division, and E. N. Group, "Technology Characterization : Microturbines," no. March, 2002.

[15] M. P. Boyce, Gas Turbine Engineering Handbook, Fourth Edi. 2012.

[16] SEMARNAT, "Informe de la situación del Medio Ambiente en México," Compend. Estadísticas Ambient. Indicadores Clave, Desempeño Ambient. y Crecim. Verde., 2015.

[17] SENER, "Prospectiva del Sector Eléctrico 2017-2031," 2017.

[18] SENER, "Reporte de Avances de Energías Limpias: Primer Semestre 2018,” 2018.

[19] Cámara De Diputados Del H Congreso de la Unión, "LEY DE TRANSICIÓN ENERGÉTICA,”pp. 1-40, 2015.

[20] IEA, “CHP and DHC Applicationse," 2019. [Online]. Available: https://www.iea.org/chp/chpanddhcapplications/.

[21] IEA, “Linking Heat and Electricity Systems," Int. Energy Agency, p. 58, 2014.

[22] IEA, "Cogeneration and District Energy," 2009.

[23] M. M. Maghanki, B. Ghobadian, G. Najafi, and R. J. Galogah, "Micro combined heat and 
power (MCHP) technologies and applications," Renew. Sustain. Energy Rev., vol. 28, pp. 510524, Dec. 2013.

[24] A. Llamas and O. Probst, "On the role of e ffi cient cogeneration for meeting Mexico 's clean energy goals," Energy Policy, vol. 112, no. October 2017, pp. 173-183, 2018.

[25] J. A. Orlando, R. of Heating, and A.-C. Engineers, Cogeneration Design Guide. American Society of Heating, Refrigerating and Air-Conditioning Engineers, 1996.

[26] A. Santamaria and H. Romero-Paredes R., "Análisis paramétrico y evaluación estadística de motores alternativos comerciales empleados para cogeneración hasta 2077 kW," FIDE, vol. 4, pp. 12-19, 2017.

[27] Fenercom, "Microcogeneracion,” p. 72, 2012.

[28] Capstone, "C30 MicroTurbine Natural Gas," Power, 2017.

[29] A. Schuster, S. Karellas, E. Kakaras, and H. Spliethoff, "Energetic and economic investigation of Organic Rankine Cycle applications," Appl. Therm. Eng., vol. 29, no. 8-9, pp. 1809-1817, Jun. 2009.

[30] J. Gómez Ribelles, Termodinámica técnica. Universidad Politécnica de Valencia, 2002.

[31] Y. A. Çengel and M. A. Boles, Termodinámica (8a. ed.). 2015.

[32] R. Karaali and I. T. Öztürk, "Thermoeconomic optimization of gas turbine cogeneration plants," Energy, vol. 80, pp. 474-485, 2015.

[33] J. L. H. Backman, "Microturbine systems for small combined heat and power (CHP) applications 7," vol. 1, 2011.

[34] E. S. Barbieri, P. R. Spina, and M. Venturini, "Analysis of innovative micro-CHP systems to meet household energy demands," Appl. Energy, vol. 97, pp. 723-733, 2012.

[35] M. J. Kim, J. H. Kim, and T. S. Kim, "Program development and simulation of dynamic operation of micro gas turbines," Appl. Therm. Eng., vol. 108, pp. 122-130, 2016.

[36] W. De Paepe, S. Abraham, P. Tsirikoglou, F. Contino, A. Parente, and G. Ghorbaniasl, "Operational Optimization of a Typical micro Gas Turbine," Energy Procedia, vol. 142, pp. 1653-1660, 2017.

[37] H. Cohen, G. F. C. Rogers, P. Straznicky, H. I. H. Saravanamuttoo, and A. Nix, Gas Turbine Theory. Pearson Education Limited, 2017.

[38] M. Moran and H. Shapiro, Fundamentals of engineering thermodynamics, vol. 91. 2017.

[39] SENER, "PRONTURARIO ESTADÍSTICO mayo 2017,” 2017.

[40] K. Rayaprolu, Boiler for Power and Process. 2009.

[41] A. Plauchú, Eficiencia en sistemas de generación y distribución de vapor, 2006th ed. México, 2006.

[42] N. O. M. semarnat-, "NOM-085-SEMARNAT-2011, Contaminación atmosférica-Niveles máximos permisibles de emisión de los equipos de combustión de calentamiento indirecto y su medición,” pp. 1-14, 2012.

[43] F. Basrawi, T. Yamada, K. Nakanishi, and S. Naing, "Effect of ambient temperature on the performance of micro gas turbine with cogeneration system in cold region," Appl. Therm. Eng., vol. 31, no. 6-7, pp. 1058-1067, 2011. 
[44] Capstone, “C65 \& C65-ICHP MicroTurbine Natural Gas,” Power, no. 4, pp. 9-10, 2017.

[45] SENER, "Lista de combustibles 2018 que se considerarán para identificar a los usuarios con un patrón de alto consumo, así como los factores para determinar las equivalencias en términos de barriles equivalentes de petróleo," 2018.

[46] N. academy of Engineering, The Hydrogen Economy: Opportunities, Costs, Barriers and R\&D Needs. 2004.

[47] A. Santamaria, H. Romero-Paredes R., and N. Segura-Pacheco, "Análisis paramétrico y evaluación estadística de las turbinas de gas comerciales empleadas para cogeneración hasta 50MW," Rev. Investig. y Desarro., vol. 2, pp. 82-91, 2016.

[48] C. Brooks, “15-100 HP Boilers Steam and Hot Water,” p. 54, 2010.

[49] H. L. Sadik Kakac, Heat Exchangers: Selection, rating, and thermal design, Third Edit. 2012.

[50] W. M. Rohsenow and J. R. Hartnett, HANDBOOK OF HEAT TRANSFER, Third edit. 1998.

[51] N. Afgan and F. Schiünder, Heat Exchangers: Design and theory sourcebook. 1974.

[52] Y. A. Çengel, Heat and Mass Transfer: Fundamentals \& Applications, Fifth Edit. 2015.

[53] J. Thome, The Heat Transfer Engineering Data Book III. 2016.

[54] Cleaver Brooks, “Termiz Soluciones térmicas Cleaver Brooks,” 2018.

[55] A. Santamaria and H. Romero-Paredes R., "Gas turbine for Micro-CHP system and heat recovery with a conventional steam boiler: Thermodynamic analysis," 2018 IEEE 38th Cent. Am. Panama Conv. (CONCAPAN XXXVIII), pp. 1-7, 2018.

[56] C. F. Curtiss and J. O. Hirschfelder, "Transport properties of multicomponent gas mixtures," J. Chem. Phys., vol. 17, no. 6, pp. 550-555, 1949.

[57] C. R. Wilke, “A viscosity equation for gas mixtures,” J. Chem. Phys., vol. 18, no. 4, pp. 517$519,1950$.

[58] R. B. Bird, W. E. Stewart, and E. N. Lightfoot, Fenômenos de Transporte, Segunda Edição. 2004.

[59] G. Xiao et al., "Recuperators for micro gas turbines: A review," Appl. Energy, vol. 197, pp. 83-99, 2017.

[60] K. Thu, B. B. Saha, K. J. Chua, and T. D. Bui, "Thermodynamic analysis on the part-load performance of a microturbine system for micro/mini-CHP applications," Appl. Energy, vol. 178, pp. 600-608, 2016.

[61] S. Bracco and F. Delfino, "A mathematical model for the dynamic simulation of low size cogeneration gas turbines within smart microgrids," Energy, vol. 119, pp. 710-723, Jan. 2017.

[62] S. Murugan and B. Horák, "A review of micro combined heat and power systems for residential applications," Renew. Sustain. Energy Rev., vol. 64, pp. 144-162, Oct. 2016.

[63] M. Gambini and M. Vellini, "High Efficiency Cogeneration: Electricity from Cogeneration in CHP Plants," Energy Procedia, vol. 81, pp. 430-439, Dec. 2015.

[64] M. Dentice d'Accadia, M. Sasso, S. Sibilio, and L. Vanoli, "Micro-combined heat and power in residential and light commercial applications," Appl. Therm. Eng., vol. 23, no. 10, pp. 12471259, Jul. 2003. 
[65] G. Comodi, L. Cioccolanti, and M. Renzi, "Modelling the Italian household sector at the municipal scale: Micro-CHP, renewables and energy efficiency," Energy, vol. 68, pp. 92-103, Apr. 2014.

[66] O. Kurata et al., "Micro gas turbine cogeneration system with latent heat storage at the University: Part I: Plan and energy flow test," Appl. Therm. Eng., vol. 65, no. 1-2, pp. 513523, Apr. 2014.

[67] G. Angrisani, A. Rosato, C. Roselli, M. Sasso, and S. Sibilio, "Experimental results of a microtrigeneration installation," Appl. Therm. Eng., vol. 38, pp. 78-90, May 2012.

[68] M. Lipošćak, N. H. Afgan, N. Duić, and M. da Graça Carvalho, "Sustainability assessment of cogeneration sector development in Croatia," Energy, vol. 31, no. 13, pp. 2276-2284, Oct. 2006. 


\section{Apéndice A}

Para el cálculo de la viscosidad y conductividad térmica de la mezcla de gases de combustión con y sin gases provenientes de una MTG se sigue la metodología esta descrita en los diagramas de flujo siguientes:

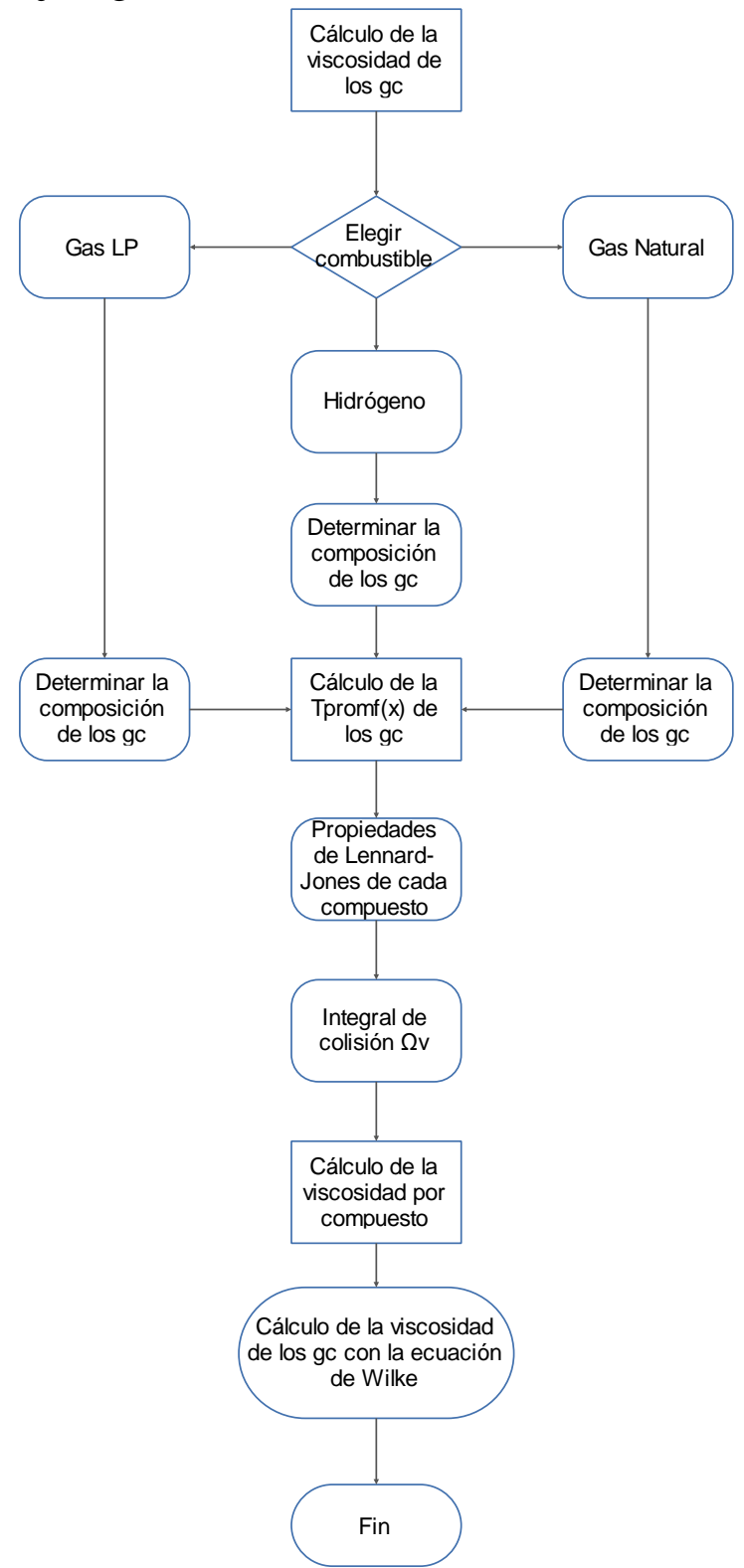

Figura 73.- Diagrama de flujo para el cálculo de la viscosidad de los gc

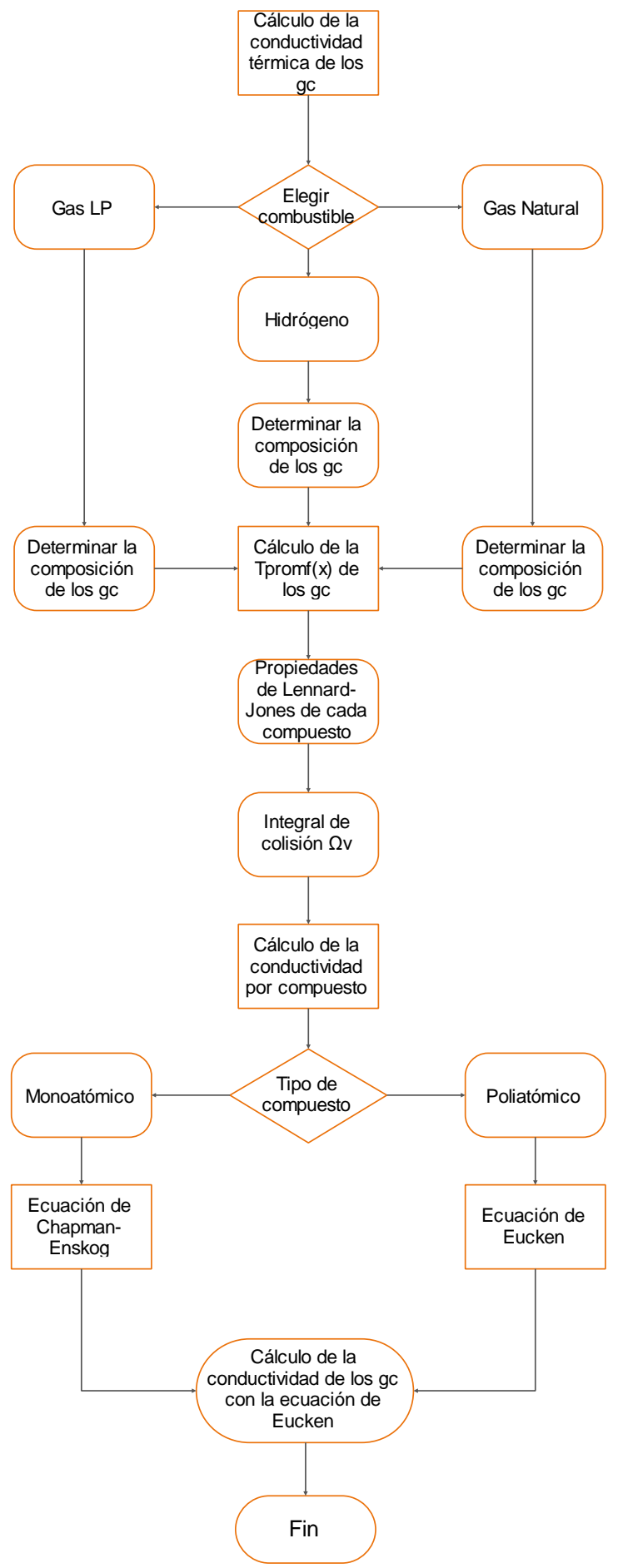

Figura 74.- Diagrama de flujo para el cálculo de la conductividad térmica de los gc

Las propiedades de Lennard-Jones, la integral de colisión $\Omega \mathrm{v}$ y demás constantes se encuentran en las tablas siguientes: 
Tabla 28.- Propiedades de Lennard-Jones determinados a partir de datos de viscosidad

\begin{tabular}{|c|c|c|c|c|}
\hline Molécula & Compuesto & $\mathrm{b}_{\mathrm{o}}, \mathrm{cm}^{3} / \mathrm{gmol}$ & $\sigma, \mathrm{A}$ & $\varepsilon_{0} / \mathrm{k},{ }^{\circ} \mathrm{K}$ \\
\hline $\mathbf{A}$ & Argón & 46,08 & 3,542 & 93,3 \\
\hline $\mathrm{He}$ & Helio & 20,95 & 2,551 & 10,22 \\
\hline $\mathrm{Kr}$ & Criptón & 61,62 & 3,655 & 178,9 \\
\hline $\mathrm{Ne}$ & Neón & 28,30 & 2,820 & 32,8 \\
\hline $\mathrm{Xe}$ & Xenón & 83,66 & 4,047 & 231,0 \\
\hline Aire & Aire & 64,50 & 3,711 & 78,6 \\
\hline $\mathrm{AsH}_{3}$ & Arsina & 89,88 & 4,145 & 259,8 \\
\hline $\mathrm{BCl}_{3}$ & Cloruro de Boro & 170,1 & 5,127 & 337,7 \\
\hline $\mathrm{BF}_{3}$ & Fluoruro de Boro & 93,35 & 4,198 & 186,3 \\
\hline $\mathrm{B}\left(\mathrm{OCH}_{2}\right)_{3}$ & Borato Metílico & 210,3 & 5,503 & 396,7 \\
\hline $\mathrm{Br}_{2}$ & Bromo & 100,1 & 4,296 & 507,9 \\
\hline $\mathrm{CCl}_{4}$ & $\begin{array}{l}\text { Tetracloruro de } \\
\text { Carbono }\end{array}$ & 265,5 & 5,947 & 322,7 \\
\hline $\mathrm{CF}_{4}$ & $\begin{array}{l}\text { Tetrafluoruro de } \\
\text { Carbono }\end{array}$ & 127,9 & 4,662 & 134,0 \\
\hline $\mathrm{CHCl}_{3}$ & Cloroformo & 197,5 & 5,389 & 340,2 \\
\hline $\mathrm{CH}_{2} \mathrm{Cl}_{2}$ & Cloruro de metileno & 148,3 & 4,898 & 356,3 \\
\hline $\mathrm{CH}_{3} \mathrm{Br}$ & Bromuro de metilo & 88,14 & 4,118 & 449,2 \\
\hline $\mathrm{CH}_{3} \mathrm{Cl}$ & Cloruro de Metilo & 92,31 & 4,182 & 350 \\
\hline $\mathrm{CH}_{3} \mathrm{OH}$ & Metanol & 60,17 & 3,626 & 481,8 \\
\hline $\mathrm{CH}_{4}$ & Metano & 66,98 & 3,758 & 148,6 \\
\hline $\mathrm{CO}$ & $\begin{array}{l}\text { Monóxido de } \\
\text { Carbono }\end{array}$ & 63,41 & 3,690 & 91,7 \\
\hline $\mathrm{COS}$ & Sulfuro de Carbonilo & 88,91 & 4,130 & 336,0 \\
\hline $\mathrm{CO}_{2}$ & Dióxido de Carbono & 77,25 & 3,941 & 195,2 \\
\hline $\mathrm{CS}_{2}$ & Disulfuro de Carbono & 113,7 & 4,483 & 467 \\
\hline $\mathrm{C}_{2} \mathrm{H}_{2}$ & Acetileno & 82,79 & 4,033 & 231,8 \\
\hline $\mathrm{C}_{2} \mathrm{H}_{4}$ & Etileno & 91,06 & 4,163 & 224,7 \\
\hline $\mathrm{C}_{2} \mathrm{H}_{6}$ & Etano & 110,7 & 4,443 & 215,7 \\
\hline $\mathrm{C}_{2} \mathrm{H}_{5} \mathrm{Cl}$ & Cloruro etílico & 148,3 & 4,898 & 300 \\
\hline $\mathrm{C}_{2} \mathrm{H}_{5} \mathrm{OH}$ & Etanol & 117,3 & 4,530 & 362,6 \\
\hline $\mathrm{C}_{2} \mathrm{~N}_{2}$ & Cianógeno & 104,7 & 4,361 & 348,6 \\
\hline $\mathrm{CH}_{3} \mathrm{OCH}_{3}$ & Eter metílico & 100,9 & 4,307 & 395,0 \\
\hline $\mathrm{CH}_{2} \mathrm{CHCH}_{3}$ & Propileno & 129,2 & 4,678 & 298,9 \\
\hline $\mathrm{CH}_{3} \mathrm{CCH}$ & Metilacetileno & 136,2 & 4,761 & 251,8 \\
\hline $\mathrm{C}_{3} \mathrm{H}_{6}$ & Ciclopropano & 140,2 & 4,807 & 248,9 \\
\hline $\mathrm{C}_{3} \mathrm{H}_{8}$ & Propano & 169,2 & 5,118 & 237,1 \\
\hline $\mathrm{n}-\mathrm{C}_{3} \mathrm{H}_{7} \mathrm{OH}$ & Alcohol n-propílico & 118,8 & 4,549 & 576,7 \\
\hline $\mathrm{CHCOCH}_{3}$ & Acetona & 122,8 & 4,600 & 560,2 \\
\hline
\end{tabular}




\begin{tabular}{|c|c|c|c|c|}
\hline Molécula & Compuesto & $\mathrm{b}_{\mathrm{o}}, \mathrm{cm}^{3} / \mathrm{gmol}$ & $\sigma, \mathrm{A}$ & $\varepsilon_{0} / \mathrm{k},{ }^{\circ} \mathrm{K}$ \\
\hline $\mathrm{CH}_{3} \mathrm{COOCH}_{3}$ & Acetato metílico & 151,8 & 4,936 & 469,8 \\
\hline $\mathrm{n}-\mathrm{C}_{4} \mathrm{H}_{10}$ & n-Butano & 130,0 & 4,687 & 531,4 \\
\hline Iso- $\mathrm{C}_{4} \mathrm{H}_{10}$ & Isobutano & 185,6 & 5,278 & 330,1 \\
\hline $\mathrm{C}_{2} \mathrm{H}_{5} \mathrm{OC}_{2} \mathrm{H}_{5}$ & Eter etílico & 231,0 & 5,678 & 313,8 \\
\hline $\mathrm{CH}_{3} \mathrm{COOC}_{2} \mathrm{H}_{5}$ & Acetato etílico & 178,0 & 5,205 & 521,3 \\
\hline $\mathrm{n}-\mathrm{C}_{2} \mathrm{H}_{12}$ & n-Pentano & 244,2 & 5,784 & 341,1 \\
\hline $\mathrm{C}\left(\mathrm{CH}_{3}\right)_{4}$ & 2,2-Dimetilpropano & 340,9 & 6,464 & 193,4 \\
\hline $\mathrm{C}_{6} \mathrm{H}_{6}$ & Benceno & 193,2 & 5,349 & 412,3 \\
\hline $\mathrm{C}_{6} \mathrm{H}_{12}$ & Ciclohexano & 298,2 & 6,182 & 297,1 \\
\hline $\mathrm{n}-\mathrm{C}_{6} \mathrm{H}_{14}$ & n-hexano & 265,7 & 5,949 & 399,3 \\
\hline $\mathrm{Cl}_{2}$ & Cloro & 94,65 & 4,217 & 316,0 \\
\hline $\mathrm{F}_{2}$ & Fluor & 47,75 & 3,357 & 112,6 \\
\hline $\mathrm{HBr}$ & Acido bromhídrico & 47,58 & 3,353 & 449 \\
\hline $\mathrm{HCN}$ & Acido cianhídrico & 60,37 & 3,630 & 569,1 \\
\hline $\mathrm{HCl}$ & Acido clorhídrico & 46,98 & 3,339 & 344,7 \\
\hline $\mathrm{HF}$ & Acido fluorhídrico & 39,37 & 3,148 & 330 \\
\hline HI & Acido yodhídrico & 94,24 & 4,211 & 288,7 \\
\hline $\mathrm{H}_{2}$ & Hidrógeno & 28,51 & 2,827 & 59,7 \\
\hline $\mathrm{H}_{2} \mathrm{O}$ & Agua & 23,25 & 2,641 & 809,1 \\
\hline $\mathrm{H}_{2} \mathrm{O}_{2}$ & Peróxido de hidrógeno & 93,24 & 4,196 & 289,3 \\
\hline $\mathrm{H}_{2} \mathrm{~S}$ & Acido sulfídrico & 60,02 & 3,623 & 301,1 \\
\hline $\mathrm{Hg}$ & Mercurio & 33,03 & 2,969 & 750 \\
\hline $\mathrm{HgBr}_{2}$ & Bromuro mercúrico & 165,5 & 5,080 & 686,2 \\
\hline $\mathrm{HgCl}_{2}$ & Cloruro mercúrico & 118,9 & 4,550 & 750 \\
\hline $\mathrm{HgI}_{2}$ & Yoduro mercúrico & 224,6 & 5,625 & 695,6 \\
\hline $\mathrm{I}_{2}$ & Yodo & 173,4 & 5,160 & 474,2 \\
\hline $\mathrm{NH}_{3}$ & Amoníaco & 30,78 & 2,900 & 558,3 \\
\hline $\mathrm{NO}$ & Oxido nítrico & 53,74 & 3,492 & 116,7 \\
\hline $\mathrm{NOCl}$ & Cloruro de nitrosilo & 87,75 & 4,112 & 395,3 \\
\hline $\mathrm{N}_{2}$ & Nitrógeno & 69,14 & 3,798 & 71,4 \\
\hline $\mathrm{N}_{2} \mathrm{O}$ & Oxido nitroso & 70,80 & 3,828 & 232,4 \\
\hline $\mathrm{O}_{2}$ & Oxígeno & 52,60 & 3,467 & 106,7 \\
\hline $\mathrm{PH}_{3}$ & Fosfina & 79,63 & 3,981 & 251,5 \\
\hline $\mathrm{SF}_{6}$ & Hexafluoruro de azufre & 170,2 & 5,128 & 222,1 \\
\hline $\mathrm{SO}_{2}$ & Dióxido de azufre & 87,75 & 4,112 & 335,4 \\
\hline $\mathrm{SiF}_{4}$ & Tetrafluoruro de silicio & 146,7 & 4,880 & 171,9 \\
\hline $\mathrm{SiH}_{4}$ & Hidruro de silicio & 85,97 & 4,084 & 207,6 \\
\hline $\mathrm{SnBr}_{4}$ & Bromuro estánico & 329,0 & 6,388 & 563,7 \\
\hline $\mathrm{UF}_{6}$ & Hexafluoruro de uranio & 268,1 & 5,967 & 236,8 \\
\hline
\end{tabular}

Tabla 29.- Valores de la integral de colisión $\Omega v$ para la viscosidad y de la función de temperatura y viscosidad.

\begin{tabular}{|c|c|c|c|c|c|}
\hline $\mathbf{k T} / \varepsilon \mathbf{0}$ & $\mathbf{\Omega v}$ & $\mathbf{f 1}(\mathbf{k T} / \mathbf{\varepsilon o})$ & $\mathbf{k T / \varepsilon \mathbf { 0 }}$ & $\mathbf{\Omega v}$ & $\mathbf{f 1 ( k T / \varepsilon o )}$ \\
\hline 0.30 & 2.785 & 0.1969 & 2.7 & 1.069 & 15.417 \\
\hline 0.35 & 2.628 & 0.2252 & 2.8 & 1.058 & 15.861 \\
\hline 0.40 & 2.492 & 0.254 & 2.9 & 1.048 & 16.298 \\
\hline 0.45 & 2.368 & 0.2834 & 3 & 1.039 & 16.728 \\
\hline 0.5 & 2.257 & 0.3134 & 3.1 & 1.030 & 17.154 \\
\hline 0.55 & 2.156 & 0.344 & 3.2 & 1.022 & 17.573 \\
\hline 0.6 & 2.065 & 0.3751 & 3.3 & 1.014 & 17.983 \\
\hline 0.65 & 1.982 & 0.4066 & 3.4 & 1.007 & 18.388 \\
\hline 0.7 & 1.908 & 0.4384 & 3.5 & 1.000 & 18.789 \\
\hline 0.75 & 1.841 & 0.4704 & 3.6 & 0.993 & 19.186 \\
\hline
\end{tabular}




\begin{tabular}{|c|c|c|c|c|c|}
\hline kT/عo & $\Omega v$ & f1(kT/عo) & kT/عo & $\Omega v$ & f1(kT/عo) \\
\hline 0.8 & 1.780 & 0.5025 & 3.7 & 0.987 & 19.576 \\
\hline 0.85 & 1.725 & 0.5346 & 3.8 & 0.981 & 19.962 \\
\hline 0.9 & 1.675 & 0.5566 & 3.9 & 0.976 & 20.343 \\
\hline 0.95 & 1.629 & 0.5985 & 4 & 0.97 & 20.719 \\
\hline 1 & 1.587 & 0.6302 & 4.1 & 0.9649 & 21.090 \\
\hline 1.05 & 1.549 & 0.6616 & 4.2 & 0.96 & 21.457 \\
\hline 1.1 & 1.514 & 0.6928 & 4.3 & 0.9553 & 21.820 \\
\hline 1.15 & 1.482 & 0.7237 & 4.4 & 0.9507 & 22.180 \\
\hline 1.2 & 1.452 & 0.7544 & 4.5 & 0.9464 & 22.536 \\
\hline 1.25 & 1.424 & 0.7849 & 4.6 & 0.9422 & 22.888 \\
\hline 1.3 & 1.399 & 0.8151 & 4.7 & 0.9382 & 23.237 \\
\hline 1.35 & 1.375 & 0.8449 & 4.8 & 0.9343 & 23.583 \\
\hline 1.4 & 1.353 & 0.8744 & 4.9 & 0.9305 & 23.926 \\
\hline 1.45 & 1.333 & 0.9026 & 5 & 0.9269 & 24.264 \\
\hline 1.5 & 1.314 & 0.9325 & 6 & 0.8963 & 2.751 \\
\hline 1.55 & 1.296 & 0.9611 & 7 & 0.8727 & 3.053 \\
\hline 1.6 & 1.279 & 0.9894 & 8 & 0.8538 & 3.337 \\
\hline 1.65 & 1.264 & 10.174 & 9 & 0.8379 & 3.607 \\
\hline 1.7 & 1.248 & 10.453 & 10 & 0.8242 & 3.866 \\
\hline 1.75 & 1.234 & 10.729 & 20 & 0.7432 & 6.063 \\
\hline 1.8 & 1.221 & 10.999 & 30 & 0.7005 & 7.880 \\
\hline 1.85 & 1.209 & 11.264 & 40 & 0.6718 & 9.488 \\
\hline 1.9 & 1.197 & 11.529 & 50 & 0.6504 & 10.958 \\
\hline 1.95 & 1.186 & 11.790 & 60 & 0.6335 & 12.324 \\
\hline 2 & 1.175 & 12.048 & 70 & 0.6194 & 13.615 \\
\hline 2.1 & 1.156 & 12.558 & 80 & 0.6076 & 14.839 \\
\hline 2.2 & 1.138 & 13.057 & 90 & 0.5973 & 16.010 \\
\hline 2.3 & 1.122 & 13.547 & 100 & 0.5882 & 17.137 \\
\hline 2.4 & 1.107 & 14.028 & 200 & 0.532 & 26.8 \\
\hline 2.5 & 1.093 & 14.501 & 400 & 0.4811 & 41.9 \\
\hline 2.6 & 1.081 & 14.962 & & & \\
\hline
\end{tabular}

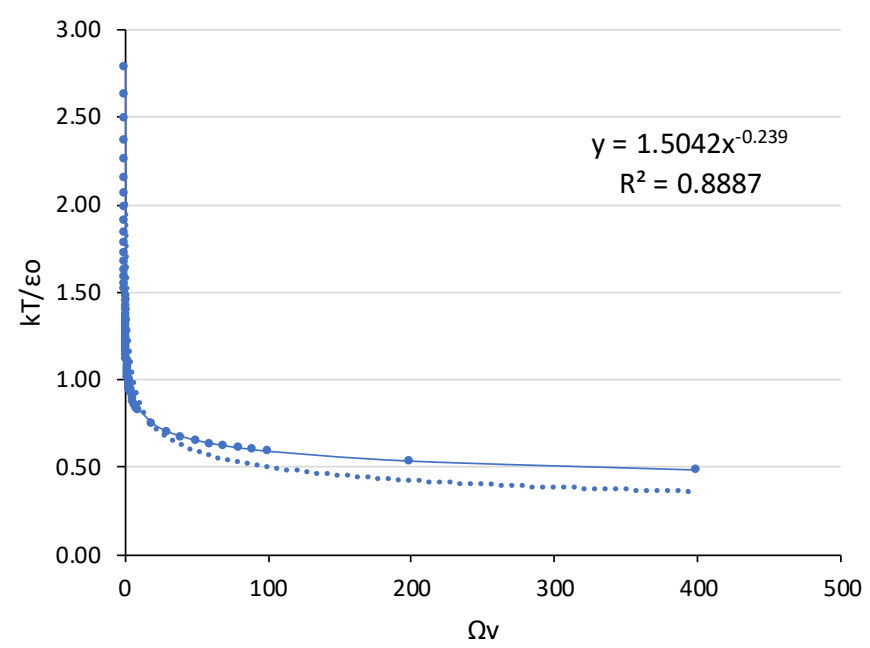

Figura 75.- Integral de colisión $\Omega v$ para la viscosidad y de la función de temperatura y viscosidad. 


\section{Apéndice B}

Tabla 30.- Capacidad calorífica de diferentes compuestos.

Calores específicos de gas ideal de varios gases comunes (conclusión)

c) Como una función de la temperatura

$$
\bar{c}_{p}=a+b T+c T^{2}+d T^{3}
$$

( $T$ en $\mathrm{K}, c_{p}$ en $\mathrm{kJ} / \mathrm{kmol} \cdot \mathrm{K}$ )

\begin{tabular}{|c|c|c|c|c|c|c|c|c|}
\hline \multirow[b]{2}{*}{ Sustancia } & \multirow[b]{2}{*}{ Fórmula } & \multirow[b]{2}{*}{$a$} & \multirow[b]{2}{*}{$b$} & \multirow[b]{2}{*}{ c } & \multirow[b]{2}{*}{$d$} & \multirow{2}{*}{$\begin{array}{l}\text { Rango de } \\
\text { temp., K }\end{array}$} & \multicolumn{2}{|c|}{$\%$ de error } \\
\hline & & & & & & & Máx. & Prom. \\
\hline Acetileno & $\mathrm{C}_{2} \mathrm{H}_{2}$ & 21.8 & $9.2143 \times 10^{-2}$ & $-6.527 \times 10^{-5}$ & $18.21 \times 10^{-9}$ & $273-1500$ & 1.46 & 0.59 \\
\hline Aire & - & 28.11 & $0.1967 \times 10^{-2}$ & $0.4802 \times 10^{-5}$ & $-1.966 \times 10^{-9}$ & $273-1800$ & 0.72 & 0.33 \\
\hline Amoniaco & $\mathrm{NH}_{3}$ & 27.568 & $2.5630 \times 10^{-2}$ & $0.99072 \times 10^{-5}$ & $-6.6909 \times 10^{-9}$ & $273-1500$ & 0.91 & 0.36 \\
\hline Azufre & $\mathrm{S}_{2}$ & 27.21 & $2.218 \times 10^{-2}$ & $-1.628 \times 10^{-5}$ & $3.986 \times 10^{-9}$ & $273-1800$ & 0.99 & 0.38 \\
\hline Benceno & $\mathrm{C}_{6} \mathrm{H}_{6}$ & -36.22 & $48.475 \times 10^{-2}$ & $-31.57 \times 10^{-5}$ & $77.62 \times 10^{-9}$ & $273-1500$ & 0.34 & 0.20 \\
\hline i-Butano & $\mathrm{C}_{4} \mathrm{H}_{10}$ & -7.913 & $41.60 \times 10^{-2}$ & $-23.01 \times 10^{-5}$ & $49.91 \times 10^{-9}$ & $273-1500$ & 0.25 & 0.13 \\
\hline$n$-Butano & $\mathrm{C}_{4} \mathrm{H}_{10}$ & 3.96 & $37.15 \times 10^{-2}$ & $-18.34 \times 10^{-5}$ & $35.00 \times 10^{-9}$ & $273-1500$ & 0.54 & 0.24 \\
\hline Cloruro de & & & & & & & & \\
\hline hidrógeno & $\mathrm{HCl}$ & 30.33 & $-0.7620 \times 10^{-2}$ & $1.327 \times 10^{-5}$ & $-4.338 \times 10^{-9}$ & $273-1500$ & 0.22 & 0.08 \\
\hline $\begin{array}{l}\text { Dióxido de } \\
\text { azufre }\end{array}$ & $\mathrm{SO}_{2}$ & 25.78 & $5.795 \times 10^{-2}$ & $-3.812 \times 10^{-5}$ & $8.612 \times 10^{-9}$ & $273-1800$ & 0.45 & 0.24 \\
\hline $\begin{array}{l}\text { Dióxido de } \\
\text { carbono }\end{array}$ & $\mathrm{CO}_{2}$ & 22.26 & $5.981 \times 10^{-2}$ & $-3.501 \times 10^{-5}$ & $7.469 \times 10^{-9}$ & $273-1800$ & 0.67 & 0.22 \\
\hline $\begin{array}{l}\text { Dióxido de } \\
\text { nitrógeno }\end{array}$ & $\mathrm{NO}_{2}$ & 22.9 & $5.715 \times 10^{-2}$ & $-3.52 \times 10^{-5}$ & $7.87 \times 10^{-9}$ & $273-1500$ & 0.46 & 0.18 \\
\hline Etano & $\mathrm{C}_{2} \mathrm{H}_{6}$ & 6.900 & $17.27 \times 10^{-2}$ & $-6.406 \times 10^{-5}$ & $7.285 \times 10^{-9}$ & $273-1500$ & 0.83 & 0.28 \\
\hline Etanol & $\mathrm{C}_{2} \mathrm{H}_{6} \mathrm{O}$ & 19.9 & $20.96 \times 10^{-2}$ & $-10.38 \times 10^{-5}$ & $20.05 \times 10^{-9}$ & $273-1500$ & 0.40 & 0.22 \\
\hline Etileno & $\mathrm{C}_{2} \mathrm{H}_{4}$ & 3.95 & $15.64 \times 10^{-2}$ & $-8.344 \times 10^{-5}$ & $17.67 \times 10^{-9}$ & $273-1500$ & 0.54 & 0.13 \\
\hline n-Hexano & $\mathrm{C}_{6} \mathrm{H}_{14}$ & 6.938 & $55.22 \times 10^{-2}$ & $-28.65 \times 10^{-5}$ & $57.69 \times 10^{-9}$ & $273-1500$ & 0.72 & 0.20 \\
\hline Hidrógeno & $\mathrm{H}_{2}$ & 29.11 & $-0.1916 \times 10^{-2}$ & $0.4003 \times 10^{-5}$ & $-0.8704 \times 10^{-9}$ & $273-1800$ & 1.01 & 0.26 \\
\hline Metano & $\mathrm{CH}_{4}$ & 19.89 & $5.024 \times 10^{-2}$ & $1.269 \times 10^{-5}$ & $-11.01 \times 10^{-9}$ & $273-1500$ & 1.33 & 0.57 \\
\hline Metanol & $\mathrm{CH}_{4} \mathrm{O}$ & 19.0 & $9.152 \times 10^{-2}$ & $-1.22 \times 10^{-5}$ & $-8.039 \times 10^{-9}$ & $273-1000$ & 0.18 & 0.08 \\
\hline $\begin{array}{l}\text { Monóxido de } \\
\text { carbono }\end{array}$ & $\mathrm{CO}$ & 28.16 & $0.1675 \times 10^{-2}$ & $0.5372 \times 10^{-5}$ & $-2.222 \times 10^{-9}$ & $273-1800$ & 0.89 & 0.37 \\
\hline Nitrógeno & $\mathrm{N}_{2}$ & 28.90 & $-0.1571 \times 10^{-2}$ & $0.8081 \times 10^{-5}$ & $-2.873 \times 10^{-9}$ & $273-1800$ & 0.59 & 0.34 \\
\hline Óxido nítrico & NO & 29.34 & $-0.09395 \times 10^{-2}$ & $0.9747 \times 10^{-5}$ & $-4.187 \times 10^{-9}$ & $273-1500$ & 0.97 & 0.36 \\
\hline Óxido nitroso & $\mathrm{N}_{2} \mathrm{O}$ & 24.11 & $5.8632 \times 10^{-2}$ & $-3.562 \times 10^{-5}$ & $10.58 \times 10^{-9}$ & $273-1500$ & 0.59 & 0.26 \\
\hline Oxígeno & $\mathrm{O}_{2}$ & 25.48 & $1.520 \times 10^{-2}$ & $-0.7155 \times 10^{-5}$ & $1.312 \times 10^{-9}$ & $273-1800$ & 1.19 & 0.28 \\
\hline$n$-Pentano & $\mathrm{C}_{5} \mathrm{H}_{12}$ & 6.774 & $45.43 \times 10^{-2}$ & $-22.46 \times 10^{-5}$ & $42.29 \times 10^{-9}$ & $273-1500$ & 0.56 & 0.21 \\
\hline Propano & $\mathrm{C}_{3} \mathrm{H}_{8}$ & -4.04 & $30.48 \times 10^{-2}$ & $-15.72 \times 10^{-5}$ & $31.74 \times 10^{-9}$ & $273-1500$ & 0.40 & 0.12 \\
\hline Propileno & $\mathrm{C}_{3} \mathrm{H}_{6}$ & 3.15 & $23.83 \times 10^{-2}$ & $-12.18 \times 10^{-5}$ & $24.62 \times 10^{-9}$ & $273-1500$ & 0.73 & 0.17 \\
\hline $\begin{array}{l}\text { Trióxido de } \\
\text { azufre }\end{array}$ & & $16 \triangle 0$ & 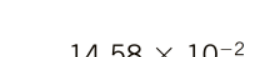 & 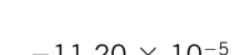 & 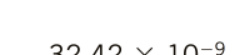 & & 20 & 013 \\
\hline $\begin{array}{l}\text { azutre } \\
\text { Agua (vapor) }\end{array}$ & $\mathrm{H}_{2} \mathrm{O}$ & $\begin{array}{l}10.40 \\
32.24\end{array}$ & $0.1923 \times 10^{-2}$ & $1.055 \times 10^{-5}$ & $\begin{array}{r}32.42 \times 10^{-9} \\
-3.595 \times 10^{-9}\end{array}$ & $\begin{array}{l}273-1300 \\
273-1800\end{array}$ & $\begin{array}{l}0.29 \\
0.53\end{array}$ & 0.24 \\
\hline
\end{tabular}

Fuente: B. G. Kyle, Chemical and Process Thermodynamics, Englewood Cliffs, Nueva Jersey, Prentice Hall, 1984. 


\section{Apéndice C}

Para el caso de la MTG C30 se tiene una pendiente positiva en la variación del coeficiente convectivo de transferencia de calor con respecto al exceso de air, debido a que predomina la variación del Nusselt, en la Figura 76 y 74 se muestra el comportamiento del Nusselt y conductividad térmica respectivamente. Se observa que la pendiente del $\mathrm{Nu}>>$ k, por lo tanto, la pendiente es positiva. En la Tabla 31 se muestran los datos con los que fueron construidas las gráficas y se observa que el Nusselt tiene una incremento máximo del $25 \%$ y la conductividad del $23 \%$. Este $2 \%$ de diferencia entre los parámetros hace la diferencia entre que sea positiva o negativa la pendiente en el incremento del coeficiente convectivo de transferencia de calor.

Tabla 31.- Variación del Nusselt y la Conductividad térmica con respecto al exceso de aire con MTG C30

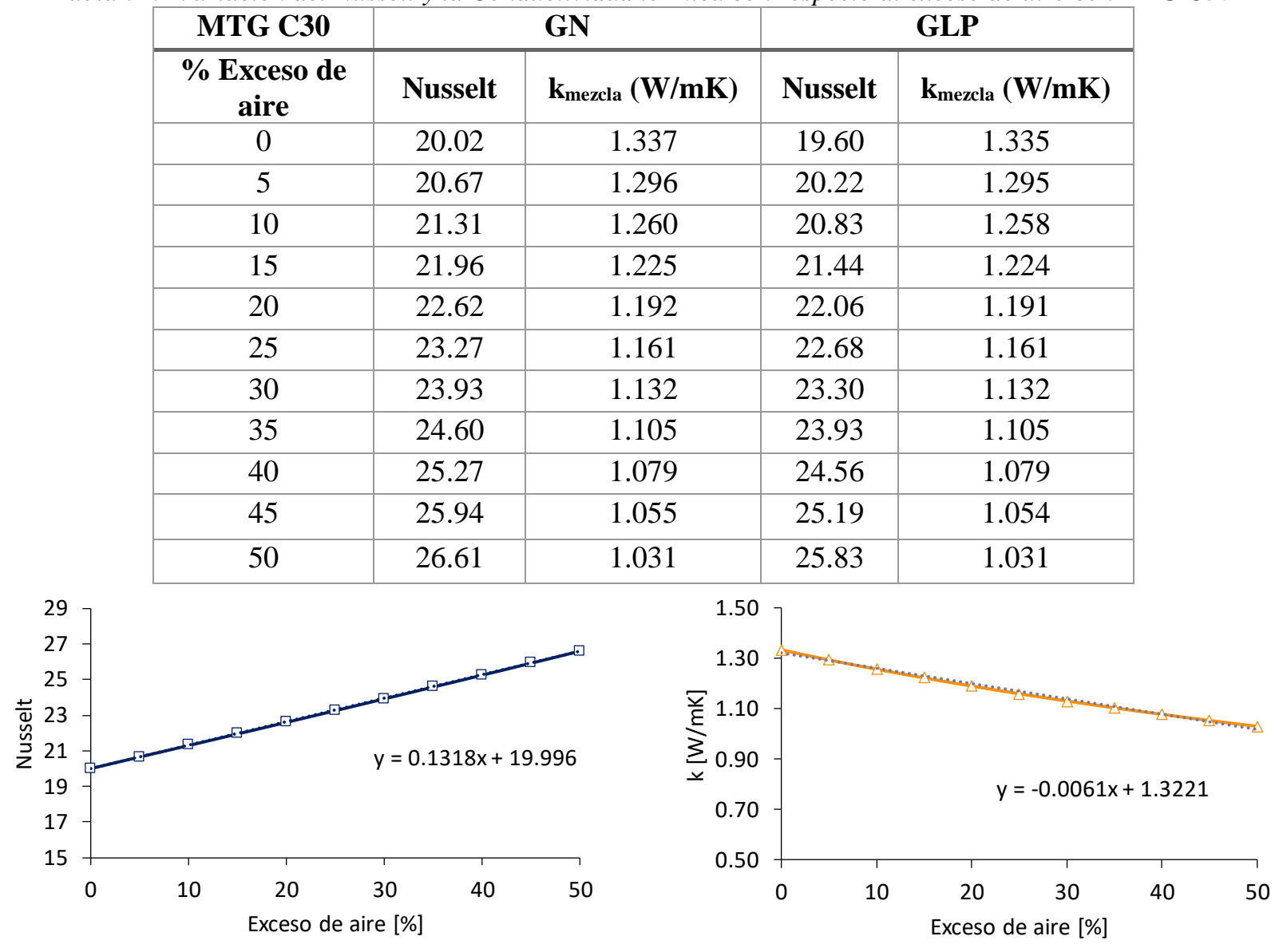

Figura 76.- Nusselt con GN en función del exceso de aire usando la MTG C30

Figura 77.- Conductividad térmica con GN en función del exceso de aire usando la MTG C30

Para el caso de la MTG C65 se tiene una pendiente negativa en la variación del coeficiente convectivo de transferencia de calor debido a que predomina la variación de la conductividad térmica de los gases. En las Figura 78 y 76 se muestra el comportamiento del Nusselt y conductividad térmica respectivamente. Se observa que la pendiente de $\mathrm{k}>>\mathrm{Nu}$, por lo tanto, la pendiente es positiva. En la Tabla 32 se muestran los datos con los que fueron construidas las gráficas y se observa que la conductividad tiene una incremento máximo del 23\% y el Nusselt del 18\%. Este 5\% de diferencia entre los parámetros hace la diferencia entre que sea positiva o negativa la pendiente en el incremento del coeficiente convectivo de transferencia de calor. 
Tabla 32.- Variación del Nusselt y la Conductividad térmica con respecto al exceso de aire con MTG 65

\begin{tabular}{|c|c|c|c|c|}
\hline MTG C65 & \multicolumn{2}{|c|}{ GN } & \multicolumn{2}{c|}{ GLP } \\
\hline $\begin{array}{c}\text { \% Exceso de } \\
\text { aire }\end{array}$ & Nusselt & $\mathbf{k}_{\text {mezcla }}(\mathbf{W} / \mathbf{m K})$ & Nusselt & $\mathbf{k}_{\text {mezcla }}(\mathbf{W} / \mathbf{m K})$ \\
\hline 0 & 21.27 & 1.337 & 20.85 & 1.335 \\
\hline 5 & 21.74 & 1.297 & 21.28 & 1.296 \\
\hline 10 & 22.19 & 1.261 & 21.70 & 1.260 \\
\hline 15 & 22.64 & 1.227 & 22.10 & 1.226 \\
\hline 20 & 23.08 & 1.194 & 22.51 & 1.194 \\
\hline 25 & 23.52 & 1.164 & 22.91 & 1.164 \\
\hline 30 & 23.96 & 1.135 & 23.31 & 1.136 \\
\hline 35 & 24.40 & 1.108 & 23.70 & 1.109 \\
\hline 40 & 24.84 & 1.082 & 24.10 & 1.083 \\
\hline 45 & 25.27 & 1.058 & 24.49 & 1.059 \\
\hline 50 & 25.71 & 1.035 & 24.87 & 1.036 \\
\hline
\end{tabular}

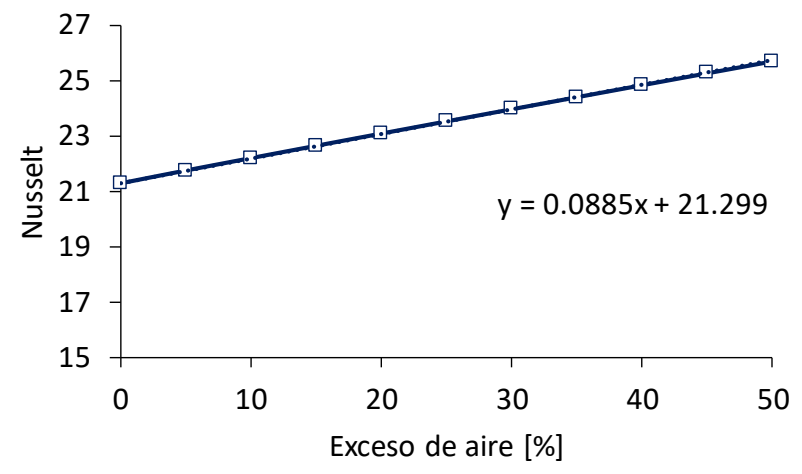

Figura 78.- Nusselt con GN en función del exceso de aire usando la MTG C65

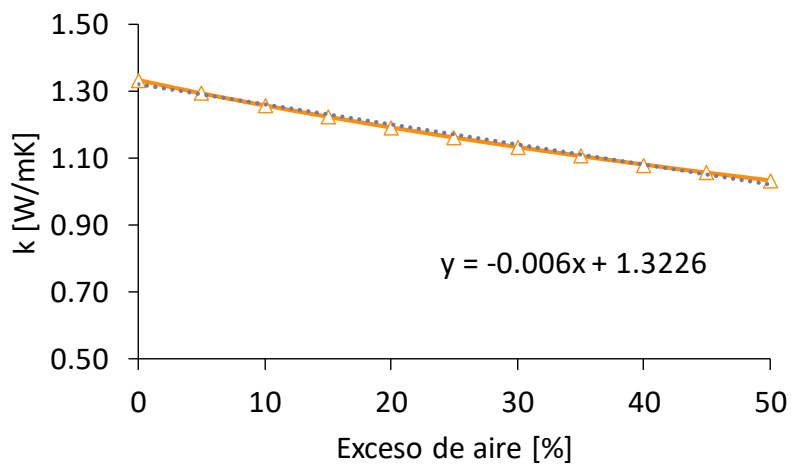

Figura 79.- Conductividad térmica con GN en función del exceso de aire usando la MTG C65 


\section{Apéndice D}

Tabla 33.- Valores de referencia de Gorenflo (1993) con $\alpha_{0}$ en $\mathrm{W} / \mathrm{m}^{2} \mathrm{~K}$ a $P_{r 0}=0.1, q_{0}=20,000 \mathrm{Wm} 2$ y $R_{p 0}=0.4 \mu m$ con $P_{c r i}$ en bar

\begin{tabular}{|c|c|c|}
\hline Fluid & Pcri & $\alpha_{0}$ \\
\hline Methane & 46 & 7000 \\
\hline Ethane & 48.8 & 4500 \\
\hline Propane & 42.4 & 4000 \\
\hline n-Butane & 38 & 3600 \\
\hline n-Pentane & 33.7 & 3400 \\
\hline i-Pentane & 33.3 & 2500 \\
\hline n-Hexane & 29.7 & 3300 \\
\hline n-Heptane & 27.3 & 3200 \\
\hline Benzene & 48.9 & 2750 \\
\hline Toluene & 41.1 & 2650 \\
\hline Diphenyl & 38.5 & 2100 \\
\hline Ethanol & 63.8 & 4400 \\
\hline n-Propanol & 51.7 & 3800 \\
\hline i-Propanol & 47.6 & 3000 \\
\hline n-Butanol & 49.6 & 2600 \\
\hline i-Butanol & 43 & 4500 \\
\hline Acetone & 47 & 3950 \\
\hline R-11 & 44 & 2800 \\
\hline R-12 & 41.6 & 4000 \\
\hline R-13 & 38.6 & 3900 \\
\hline R-13B1 & 39.8 & 3500 \\
\hline R-22 & 49.9 & 3900 \\
\hline $\mathbf{R}-23$ & 48.7 & 4400 \\
\hline R-113 & 34.1 & 2650 \\
\hline R-114 & 32.6 & 2800 \\
\hline R-115 & 31.3 & 4200 \\
\hline R-123 & 36.7 & 2600 \\
\hline R-134 ${ }^{\mathrm{a}}$ & 40.6 & 4500 \\
\hline $\mathbf{R}-152^{\mathbf{a}}$ & 45.2 & 4000 \\
\hline R-226 & 30.6 & 3700 \\
\hline R-2277 & 29.3 & 3800 \\
\hline RC318 & 28 & 4200 \\
\hline R-502 & 40.8 & 3300 \\
\hline Chloromethane & 66.8 & 4400 \\
\hline Tetrafluormethane & 37.4 & 4750 \\
\hline Hydrogen (on Cu) & 12.97 & 24000 \\
\hline Neon (on Cu) & 26.97 & 20000 \\
\hline Nitrogen (on $\mathrm{Cu}$ ) & 26.5 & 10000 \\
\hline Nitrogen (on Pt) & 34 & 7000 \\
\hline Argon (on Cu) & 34 & 8200 \\
\hline Argon (on Pt) & 49 & 6700 \\
\hline Oxygen (on Cu) & 50.5 & 9500 \\
\hline Oxygen (on Pt) & 50.5 & 7200 \\
\hline Water & 220.6 & 5600 \\
\hline Ammonia & 113 & 7000 \\
\hline Carbon Dioxide & 73.8 & 5100 \\
\hline Sulfur Hexafluoride & 37.6 & 3700 \\
\hline
\end{tabular}




\section{Anexo A}

\section{Caso de estudio 1 (Alemania 1997)}

\section{Proyecto: Complejo industrial y de servicios "Königstadt-Terrassen"}

Hoy, el complejo industrial y de servicios "Königstadt-Terrassen", en el terreno de una antigua fábrica de cerveza tradicional del mismo nombre, aloja numerosas empresas industriales, oficinas, tiendas y apartamentos en una superficie de más de 40.000 metros cuadrados. Aquí, en el distrito central de Berlin Pankow, la empresa de servicios de energía Berliner Energieagentur opera una moderna central de producción combinada de calor y electricidad y de generación combinada de calor, refrigeración y electricidad. La planta, con gas natural como combustible, se compone de dos unidades de cogeneración, dos calderas de carga punta y un refrigerador de absorción.

\section{Motivación:}

- En verano, el supermercado del edificio necesita el sistema de aire acondicionado

- Demanda de calefacción sobre todo en invierno; no se utiliza agua caliente en el edificio

- Sistema de electricidad de emergencia (220 kVA) necesario para la tecnología del edificio (ascensores, etc)

\section{Solución:}

- Inicio del proyecto:

- Sector:

- Tecnología:

- Combustible:

- Potencia instalada:

- Clasificación:

- Prod. De electricidad:

- Prod. De calor:

- Uso final de calor/frío:

- Equipo complementario:

- Ahorro de costos:

- Ahorro de $\mathrm{CO}_{2}{ }^{1}$ :

\section{7}

Comercial

2 unidades de cogeneración y un refrigerador de absorción Gas natural Unidades de cogeneración: $120 \mathrm{kWe}$ y $214 \mathrm{kWth}$ cada una. Refrig. Absorción 135 $\mathrm{kW}$

Micro-trigeneración 560 a $980 \mathrm{MWh}_{\mathrm{e}} / \mathrm{año}$ 1,100 a $1950 \mathrm{MWh}_{\text {th }} /$ año Calor para calefacción en invierno y frío para refrigeración del supermercado en verano 2 calderas de baja temp. De 1,400 kWth cada una.

Aprox. 2,500 MWh/año Aprox. 700 toneladas/año

${ }^{1}$ En comparación con generación convencional de energía Fuente: Berliner Energieagentur GmbH 
Caso de estudio 2 (California 2005)

\section{Proyecto: Hotel "The Ritz-Carlton" en San Francisco}

El proyecto de trigeneración en este hotel de 336 habitaciones, gimnasio, piscina cubierta y "vaporarios" fue implementado en octubre de 2005 y combina la generación de frío, calor y electricidad (trigeneración).

\section{Solución:}

- Inicio del proyecto:

2005

- Sector:

- Tecnología:

$-$

- Combustible:

- Potencia instalada:

- Clasificación:

- Prod. De electricidad:

- Prod. De calor:

- Prod. Frío:

- Uso final de calor/frío:

$-$

Hotel

$$
4
$$
refrigeración por absorción

Gas natural

$4 \times 60 \mathrm{kWe}$

Micro-trigeneración

No disp.

No disp.

120 ton. de refrigeración

Agua caliente y aire acondicionado

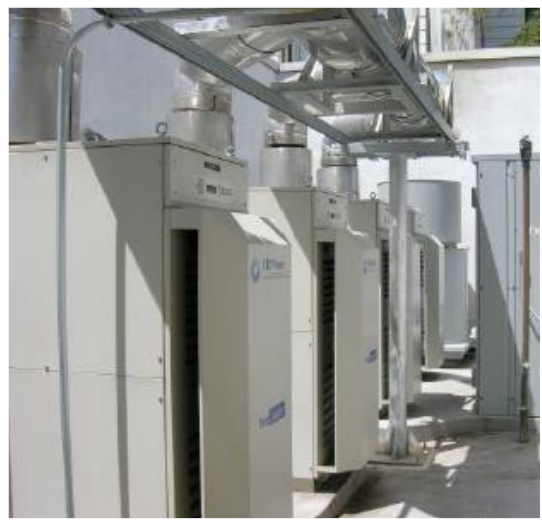

- Equipo complementario:

- Ahorro de costos:

- Ahorro de $\mathrm{CO}_{2}{ }^{1}$ :

Refrigeración por absorción

120,000 \$/año

No disp.

Fuente: Pacific Region cogeneración Application Center for California, Nevada, and Hawaii 
Caso de estudio 3 (España 2010)

\section{Proyecto: "Edificio 94 Viviendas", Colmenar Viejo - Madrid}

Este proyecto de cogeneración en un edificio de 94 viviendas, en Colmenar Viejo (Madrid), se llevó a cabo en noviembre de 2010. La cogeneración se basa en una microturbina de gas natural y una caldera de gas. La electricidad generada se cede directamente a una red de $0,4 \mathrm{kV}$. Demanda anual de electricidad: 2,145,204 kWh.

\section{Solución:}

- Inicio del proyecto:

- Sector:

- Tecnología:

- Combustible:

- Potencia instalada:

- Clasificación:

- Prod. De electricidad

- Prod. De calor:

- Uso final de calor/frío:

- Equipo complementario:

- Ahorro de costos:

- Ahorro de $\mathrm{CO}_{2}{ }^{1}$ :
2010

Público (viviendas)

1 micro-turbina

Gas natural

$65 \mathrm{kWe}$ y $120 \mathrm{kWth}$

Micro-cogeneración

No disp.

No disp.

Agua caliente

Caldera de gas (980kWth)

No disp.

No disp..

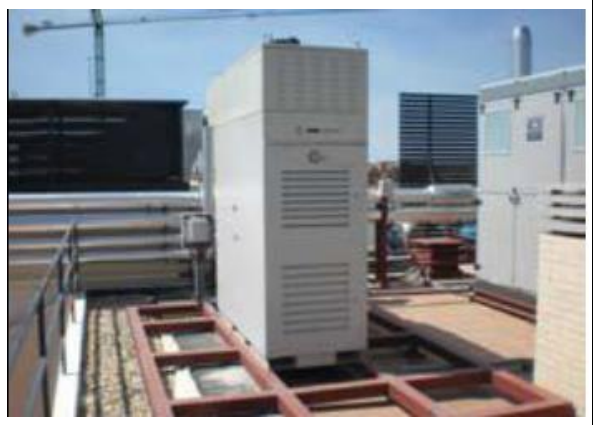

Fuente: COGEN España: quién es quién en Cogen España 2011 
Caso de estudio 4 (Reino Unido 2011)

\section{Proyecto: Residencia de estudiantes Mansfield Halls (Reading)}

La residencia Mansfield Halls, cerca de Reading, ofrece alojamiento a estudiantes de la University of Reading. Los bloques A a F del complejo albergan un total de 457 dormitorios, y el bloque $\mathrm{G}$ aloja 147 dormitorios y 14 pequeños apartamentos.

- Demanda anual de electricidad: 2,145,204 kWh

- Precio de la electricidad: $11.46 ф \mathrm{GBP} / \mathrm{kWh}$

- Consumo anual de gas: 575,293 kWh

- Precio del gas: $3.10 ф \mathrm{GBP} / \mathrm{kWh}$

\section{Reto:}

Establecer un sistema de agua caliente para uso doméstico que reduzca el consumo de energía y las emisiones de $\mathrm{CO} 2$ y, así, apoyar la estrategia de sostenibilidad de la universidad. El sistema debía, además, ser altamente flexible para responder a una demanda de agua caliente muy variable.

\section{Solución:}

- Inicio del proyecto:

- Sector:

- Tecnología:

- Combustible:

- Potencia instalada:

- Clasificación:

- Prod. De electricidad:

- Prod. De calor:

- Uso final de calor/frío:

- Equipo complementario:

- Ahorro de costos:

- Ahorro de $\mathrm{CO}_{2}{ }^{1}$ :
2011

Público (Universidad)

3 unidades de cogeneración

Gas natural

$5 \mathrm{kWe}$ y $30 \mathrm{kWth}$

Micro-cogeneración

No disp.

No disp.

Agua caliente

3 módulos de 1,000 litros de almacenamiento de calor Aprox. 29,000 GBP/año Aprox. 105 toneladas/año

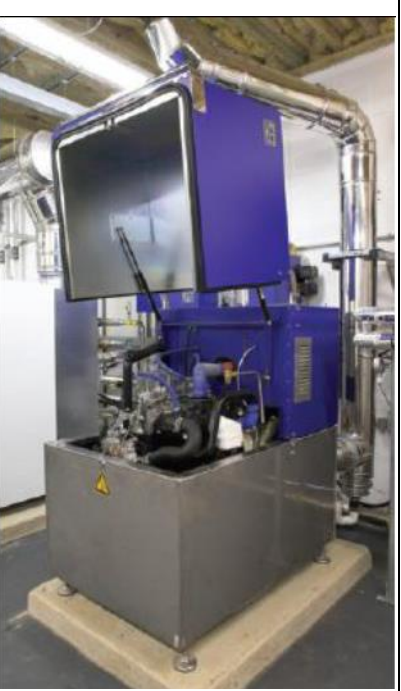

${ }^{1}$ En comparación con generación convencional de energía Fuente: http://www.chpa.co.uk/mansfield-halls_952.html 
Tabla 34.- Antecedentes de micro cogeneración en el mundo.

\begin{tabular}{|c|c|c|}
\hline Autor & Descripción & Ref. \\
\hline $\begin{array}{l}\text { Stefano } \\
\text { Bracco }\end{array}$ & $\begin{array}{l}\text { El documento describe el modelo matemático que se ha desarrollado para simular en condiciones de diseño y } \\
\text { transitorias la operación de una micro-turbina de cogeneración de } 65 \mathrm{~kW}_{\text {el }} \text { instalada dentro de una microred } \\
\text { inteligente. El documento informa las principales ecuaciones del modelo, centrándose en la arquitectura del } \\
\text { simulador y el sistema de control de micro-turbina; además, también se informan los resultados más significativos } \\
\text { derivados de la fase de validación, refiriéndose a la microturbina instalada en la Microrred Smart Polygeneration } \\
\text { del Campus de Savona en la Universidad de Génova en Italia }\end{array}$ & [61] \\
\hline $\begin{array}{l}\text { Marco } \\
\text { Gambini }\end{array}$ & $\begin{array}{l}\text { En este trabajo los autores han analizado los diferentes tipos de plantas en operación en Italia y han calculado el } \\
\text { porcentaje de electricidad a partir de la cogeneración de cada tipo de planta, teniendo en cuenta los parámetros de } \\
\text { funcionamiento típicos. El resultado de este documento puede, por lo tanto, ser una herramienta valiosa para los } \\
\text { operadores del sector de la cogeneración con el fin de identificar la idoneidad de una inversión en este sector. }\end{array}$ & {$[63]$} \\
\hline $\begin{array}{l}\text { M. Dentice } \\
\text { d'Accadia }\end{array}$ & $\begin{array}{l}\text { Este artículo trata de la aplicación de la micro-cogeneración (potencia eléctrica }<15 \mathrm{~kW} \text { ) a usuarios de pequeña } \\
\text { escala (aplicaciones residenciales y comerciales ligeras). Se describe la instalación de prueba diseñada y construida } \\
\text { para evaluar el rendimiento del sistema micro-CHP y el modo de funcionamiento óptimo para que coincida con } \\
\text { las cargas térmicas y eléctricas identificadas del usuario. }\end{array}$ & [64] \\
\hline $\begin{array}{l}\text { Gabriele } \\
\text { Comodi }\end{array}$ & $\begin{array}{l}\text { Este estudio investiga el potencial de la eficiencia energética, las energías renovables y la micro-cogeneración para } \\
\text { reducir el consumo de los hogares en un pueblo italiano medio y analiza el alcance de las políticas locales } \\
\text { municipales. Modelaron dos escenarios de energía a largo plazo (hasta 2030) utilizando el modelo de generador } \\
\text { MarkAL-TIMES: BAU (negocios habituales), que es el escenario de referencia, y EHS (sector doméstico } \\
\text { ejemplar), que implica objetivos de penetración para energías renovables y micro -cogeneración. Este estudio } \\
\text { encuentra que las tecnologías de micro-CHP con menores relaciones de potencia-calor (principalmente motores } \\
\text { Stirling y micro-turbinas) }\end{array}$ & {$[65]$} \\
\hline
\end{tabular}




\begin{tabular}{|c|c|c|}
\hline $\begin{array}{l}\text { Osamu } \\
\text { Kurata }\end{array}$ & $\begin{array}{l}\text { El Instituto de Investigación de Tecnología Energética (ETRI) del Instituto Nacional de Ciencia y Tecnología } \\
\text { Industrial Avanzada (AIST) demostró un sistema de cogeneración de turbina de gas micro con almacenamiento } \\
\text { de calor latente en la Universidad de Sapporo City. Como no había habido un sistema de cogeneración con el } \\
\text { almacenamiento de calor latente en condiciones de servicio, este sistema fue la primera demostración. El uso } \\
\text { adecuado del sistema de almacenamiento de calor latente ahorrará energía de manera económica, almacenará alta } \\
\text { densidad de energía, reducirá las emisiones de GEI y ahorrará costos operativos. }\end{array}$ & [66] \\
\hline G. Angrisani & $\begin{array}{l}\text { En el Laboratorio de Control del Medio Ambiente Construido de la Seconda Università degli Studi di Napoli, se } \\
\text { ha instalado un micro-cogenerador basado en un motor de combustión interna alternativo impulsado por gas natural } \\
\text { que se puede acoplar con un sistema de absorción termoquímica para investigar experimentalmente en el sitio las } \\
\text { prestaciones del sistema de micro-cogeneración y el sistema de micro-trigeneración en condiciones reales de } \\
\text { funcionamiento. Este artículo trata de la descripción de la planta experimental y los resultados de las primeras } \\
\text { pruebas experimentales. }\end{array}$ & {$[67]$} \\
\hline $\begin{array}{l}\text { Marko } \\
\text { Liposcak }\end{array}$ & $\begin{array}{l}\text { El documento presenta un intento de evaluar la sostenibilidad del desarrollo futuro del sector de cogeneración } \\
\text { croata. La evaluación de sostenibilidad requiere una evaluación multicriterio de los escenarios específicos que } \\
\text { deben tomarse en consideración. A este respecto, se tienen en cuenta tres escenarios del desarrollo futuro del sector } \\
\text { de cogeneración croata y para cada uno de ellos se definen y calculan indicadores de sostenibilidad ambiental, } \\
\text { social y económica. }\end{array}$ & [68] \\
\hline
\end{tabular}




\section{Anexo B}

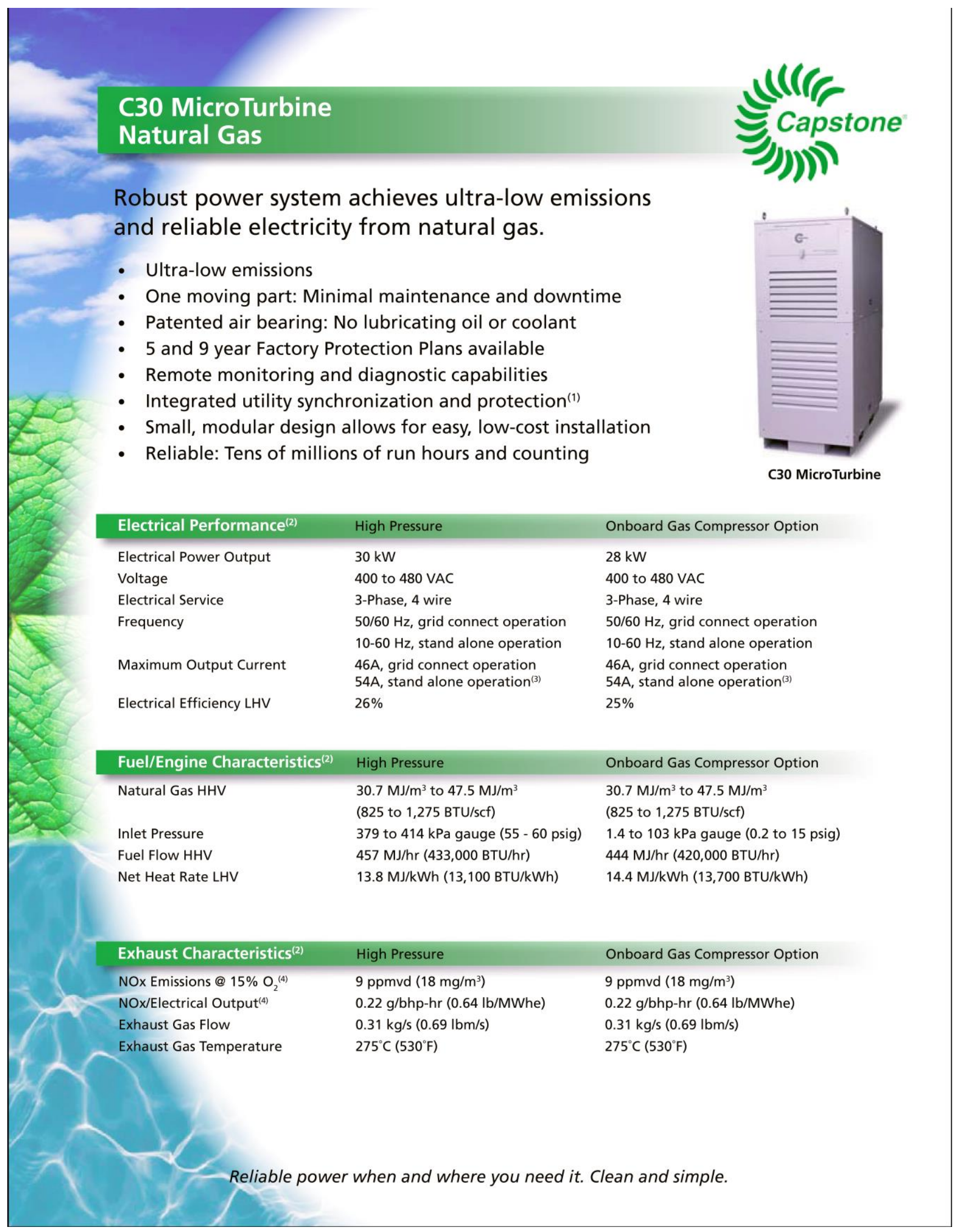




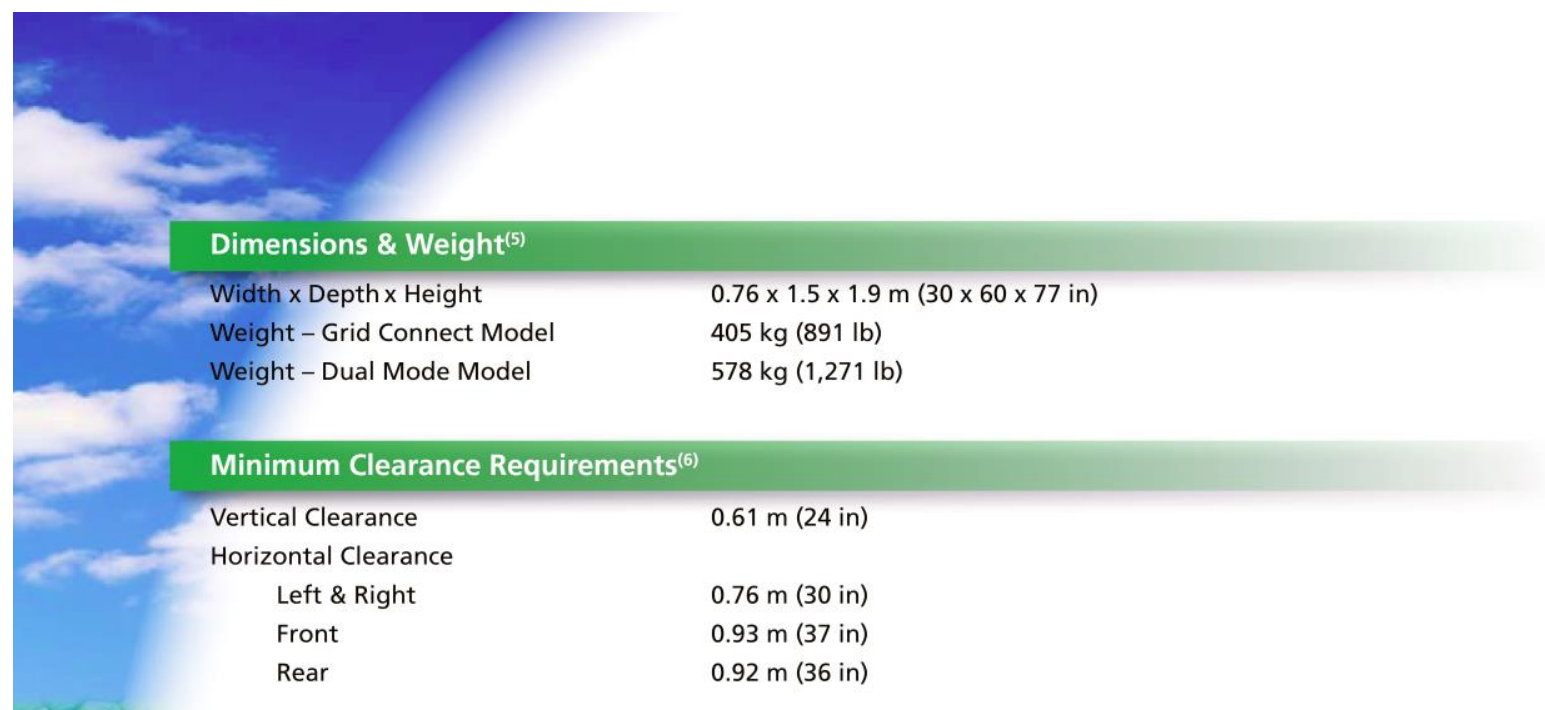

\section{Sound Levels}

Acoustic Emissions at Full Load Power

Nominal at $10 \mathrm{~m}(33 \mathrm{ft})$

$65 \mathrm{dBA}$

\section{Certifications}

- Certified to UL 2200 for stand alone natural gas operation (UL files AU2687, E209370)

- Materials Equipment Acceptance (MEA) approval for New York City

- Models available with optional equipment for CE Marking
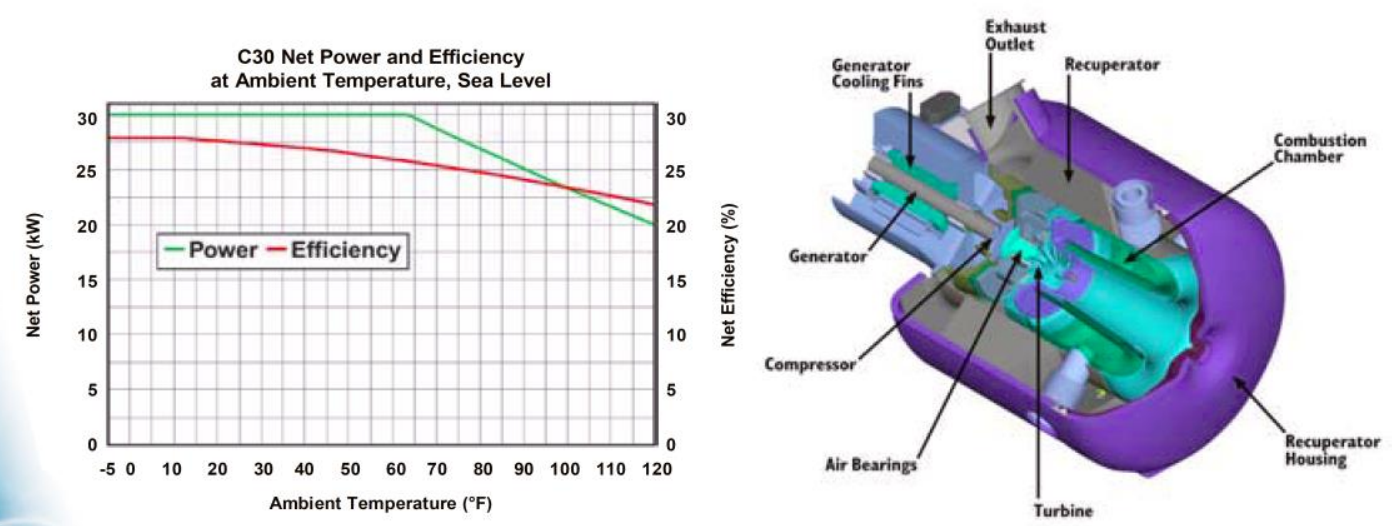

(1) Some utilities may require additional equipment for grid interconnectivity

2) Nominal full power performance at ISO conditions: $59^{\circ} \mathrm{F}, 14.696 \mathrm{psia}, 60 \% \mathrm{RH}$

(3) With linear load

Exhaust issions for standard Natural Gas at $39.4 \mathrm{MJ} / \mathrm{Nm}^{3}$ (1,000 BTU/scf) (HHV)

(5) Approximate dimensions and weights

Specifications are nuirements may increase due to local code considerations

ted and are subject to change without notice.

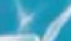

21211 Nordhoff Street • Chatsworth • CA • $91311 \bullet 877.716 .2929 \bullet 818.407 .3770 \bullet$ www.capstoneturbine.com

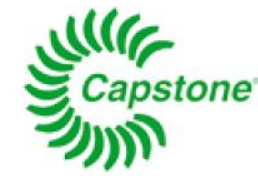

() 2008 Capstone Turbine Corporation. 10/08 Capstone P/N 331031B 


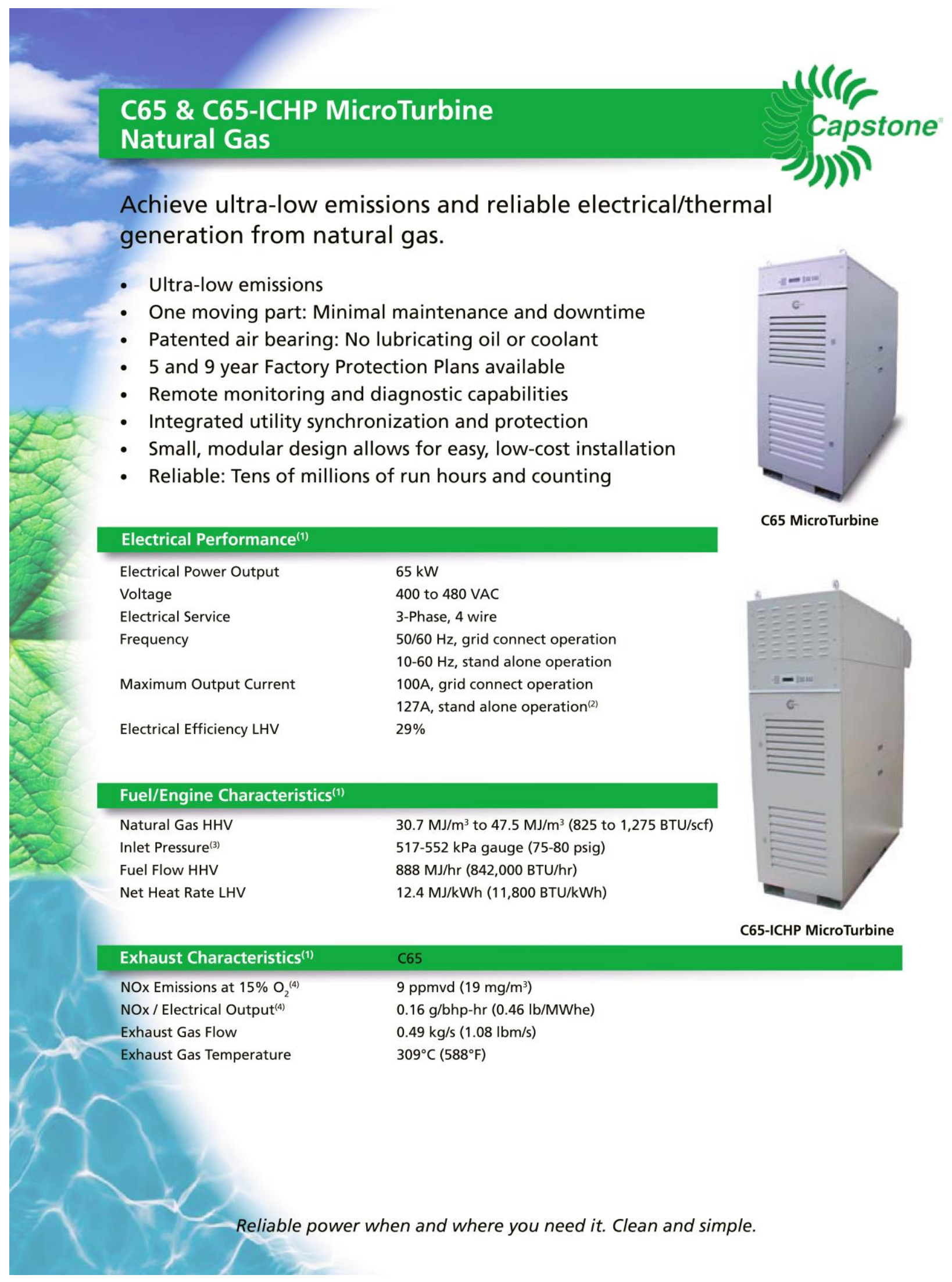




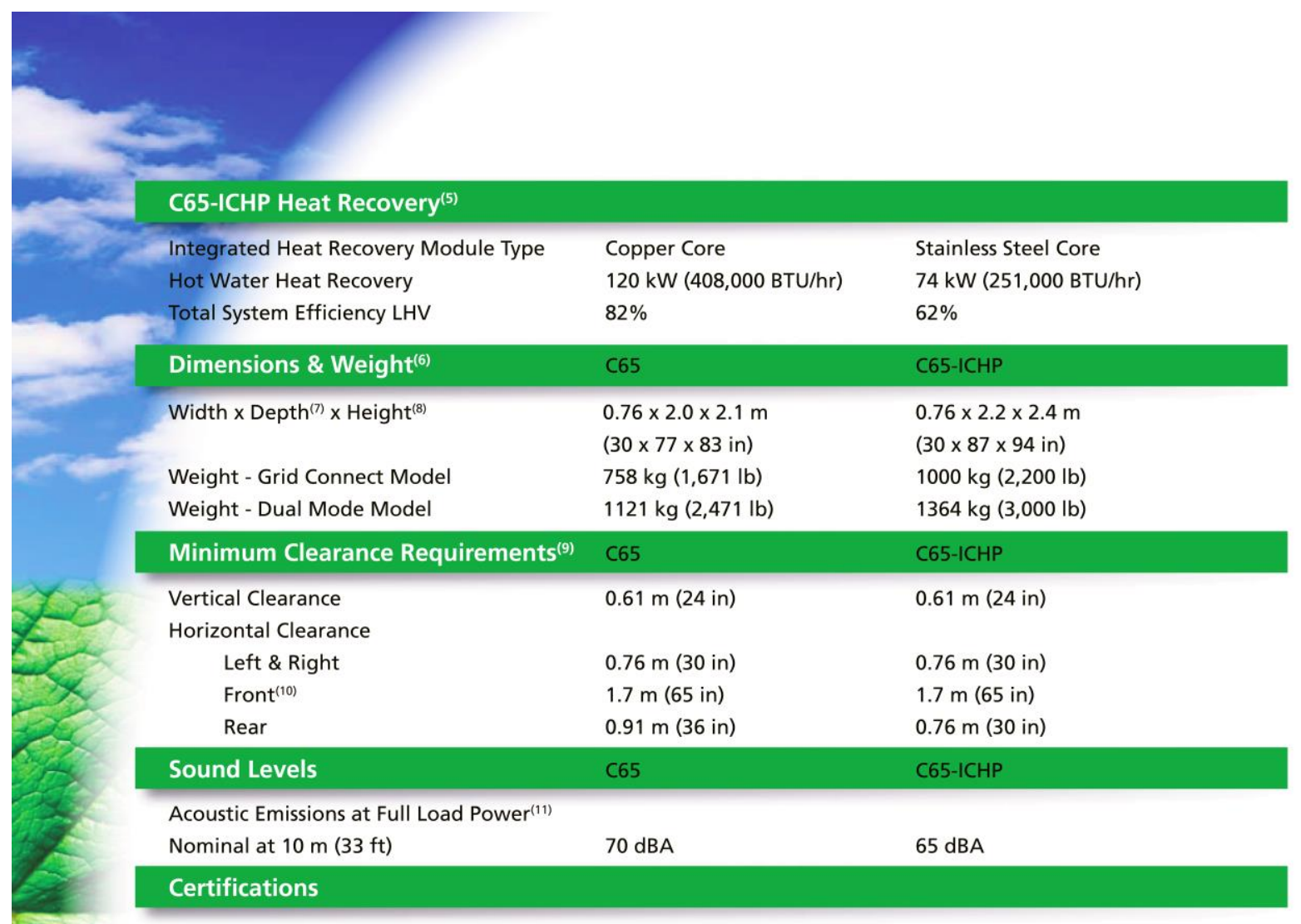

- Certified to UL 2200 and UL 1741 for natural gas operation (UL files AU2687, E209370)

- Complies with IEEE 1547 and meets statewide utility interconnection requirements for California Rule 21 and the New York State Public Service Commission

- Materials Equipment Acceptance (MEA) approval for New York City

- Models available with optional equipment for CE Marking

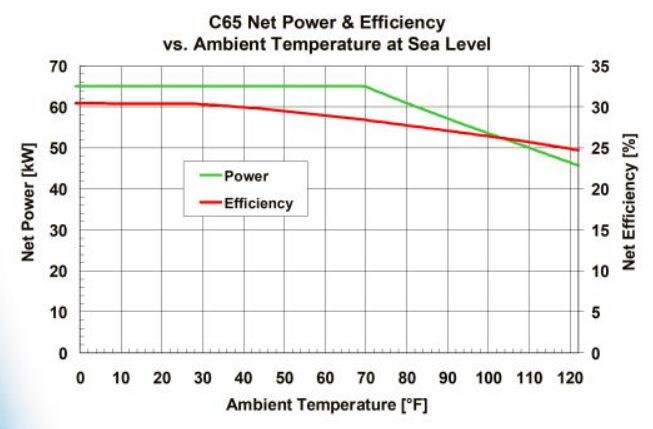

(1) Nominal full power performance at ISO conditions: $59^{\circ} \mathrm{F}, 14.696 \mathrm{psia}, 60 \% \mathrm{RH}$

(2) With linear load

Inlet pressure for standard natural gas at $39.4 \mathrm{MJ}^{\mathrm{N} \mathrm{Nm}^{3}}(1,000 \mathrm{BUT} / \mathrm{scf})$ (HHV)

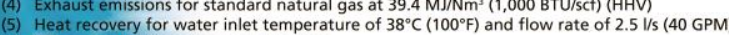

(6) Approximate dimensions and weights

(7) Depth includes 10 inch extension for the heat recovery module rain hood on ICHP versions

(8) Height dimensions are to the roof line. Exhaust outlet extends at least 7 inches above the roof line

(9) Clearance requirements may increase due to local code consideration

(10) Dual Mode MicroTurbine configuration for Battery Removal clearance

(11) The optional acoustic inlet hood kit can reduce acoustic emissions at the front of the MicroTurbine by up to $5 \mathrm{dBA}$

Specifications are not warranted and are subject to change without notice.

21211 Nordhoff Stre

(2008 20 stone Turbine Corporation

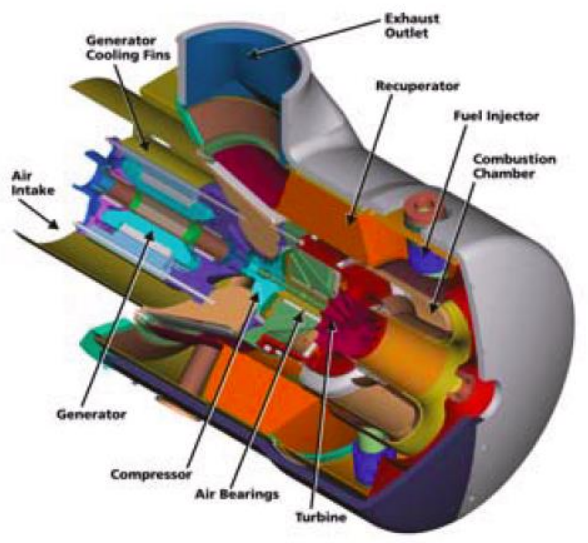

(2008 Capstone Turbine Corporation. 10/08 Capstone P/N 331035B

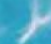




\section{Anexo C}

Technical datasheet

Gas turbine exhaust burner DD(Z)G-GTM

SAACKE

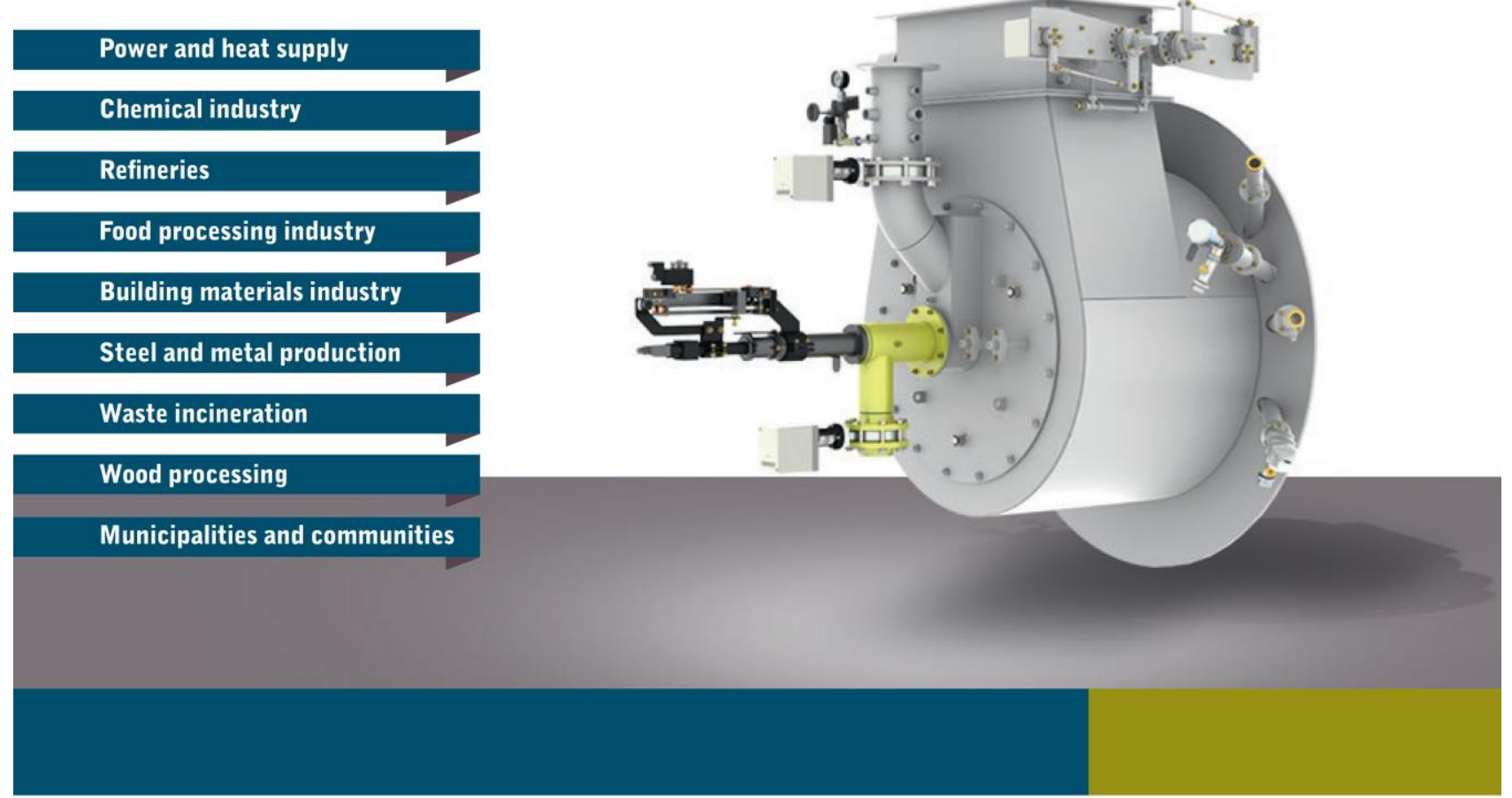

\section{Gas turbine exhaust burner DD(Z)G-GTM}

SAACKE Micro-CHP-System: energy-efficient electrical power and heat generation

Until now, small to medium-scale combined heat and power (CHP) generation was usually realized in cogeneration plants, which are unfortunately very maintenance-intensive. As an alternative, SAACKE is offering an extremely low-maintenance special burner from the DD(Z)G-GTM line for these types of CHP projects. A gas turbine generates 50 to $1,000 \mathrm{~kW}$ electrical power. The high-energy turbine gas, which is fired in the downstream heat generator, is utilized up to 100 percent as combustion air in the DD(Z)G-GTM SAACKE gas turbine exhaust burner. Depending on design and configuration of the heat generator, this type of CHP system generates heat, steam or hot water - and produces valuable electrical energy. This combination of turbine and burner always guarantees continuously low emission levels which are reliably below requirements across the entire range of $\mathrm{EU}$ regulations.

\section{Technical data: DD(Z)G-GTM}

\begin{tabular}{ll}
\hline Areas of application & $\begin{array}{l}\text { Shell boilers, water-tube boilers, } \\
\text { thermal oil heaters, hot gas generators }\end{array}$ \\
\hline Burner capacity (max.) & $2-20 \mathrm{MW}$ \\
\hline $\begin{array}{l}\text { Combustion air } \\
\text { temperature }\end{array}$ & $5-300^{\circ} \mathrm{C}$ \\
\end{tabular}


SAACKE

\section{DD(Z)G-GTM dimensions}

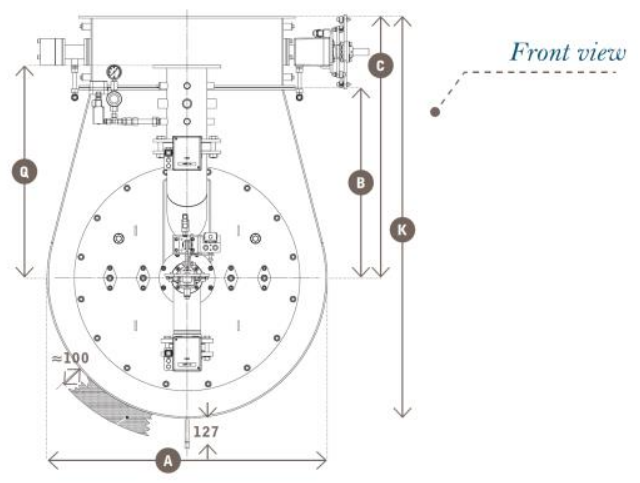

Burner size (mm)

\begin{tabular}{lrrrrrrrr}
\hline Size & A & B & C & D & E & $\begin{array}{c}\text { F } \\
\text { DozG }\end{array}$ & $\begin{array}{c}\text { GDG GozG } \\
\text { DDGG }\end{array}$ \\
\hline $\mathbf{2}$ & 630 & 450 & 900 & 240 & 475 & 1.185 & 320 & 555 \\
\hline $\mathbf{5}$ & 780 & 560 & 860 & 335 & 550 & 1.220 & 350 & 585 \\
\hline $\mathbf{8}$ & 980 & 700 & 1.000 & 395 & 650 & 1.270 & 375 & 665 \\
\hline $\mathbf{1 3}$ & 1.180 & 800 & 1.100 & 520 & 800 & 1.270 & 375 & 665 \\
\hline $\mathbf{2 0}$ & 1.450 & 1.000 & 1.300 & 680 & 850 & 1.270 & 375 & 665 \\
\hline
\end{tabular}

\begin{tabular}{lrrrrrrrr} 
& $\begin{array}{c}\text { H } \\
\text { DDG/DOZG }\end{array}$ & DDGG & K & L & $\begin{array}{c}\text { M } \\
\text { DOG }\end{array}$ & DDZG & DDGG & N \\
\hline $\mathbf{2}$ & 150 & 150 & 1.215 & 161 & 1.195 & 1.860 & 1.195 & 177 \\
\hline $\mathbf{5}$ & 150 & 150 & 1.250 & 161 & 1.300 & 1.970 & 1.300 & 246 \\
\hline $\mathbf{8}$ & 275 & 200 & 1.490 & 161 & 1.440 & 2.120 & 1.440 & 289 \\
\hline $\mathbf{1 3}$ & 275 & 200 & 1.690 & 161 & 1.590 & 2.270 & 1.590 & 289 \\
\hline $\mathbf{2 0}$ & 275 & 200 & 2.025 & 161 & 1.640 & 2.320 & 1.640 & 289
\end{tabular}

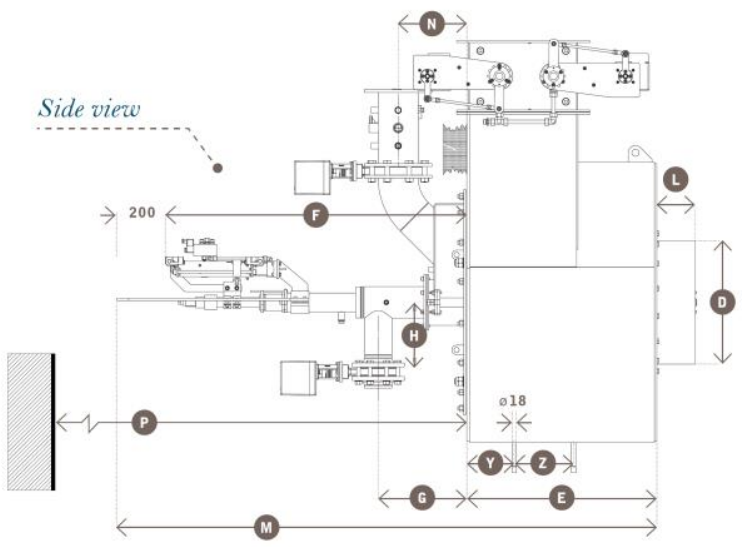

\begin{tabular}{lccccrr} 
& DDG & $\begin{array}{c}\text { P } \\
\text { DDZ6 }\end{array}$ & DOGG & Q & \multicolumn{1}{c}{$\mathbf{Y}$} & $\mathbf{Z}$ \\
\hline $\mathbf{2}$ & 1.150 & 1.860 & 1.410 & 860 & 50 & 80 \\
\hline $\mathbf{5}$ & 1.275 & 2.000 & 1.550 & 950 & 80 & 165 \\
\hline $\mathbf{8}$ & 1.425 & 2.200 & 1.740 & 896 & 100 & 230 \\
\hline $\mathbf{1 3}$ & 1.575 & 2.350 & 1.890 & 896 & 130 & 250 \\
\hline $\mathbf{2 0}$ & 1.625 & 2.400 & 1.940 & 896 & 150 & 300 \\
\hline
\end{tabular}

\section{Burner weight $(\mathrm{kg})$}

\begin{tabular}{lrrr}
\hline Size & DDG-GTM & DDGG-GTM & DDZG-GTM \\
\hline $\mathbf{2}$ & 450 & 470 & 500 \\
\hline $\mathbf{5}$ & 510 & 530 & 560 \\
\hline $\mathbf{8}$ & 760 & 790 & 810 \\
\hline $\mathbf{1 3}$ & 1.000 & 1.030 & 1.050 \\
\hline $\mathbf{2 0}$ & 1.420 & 1.450 & 1.470 \\
\hline
\end{tabular}

\section{Product notes}

$\searrow$ Easy retrofitting of existing heat generators as well as short downtime for installation and commissioning

$\searrow$ Long maintenance intervals for turbine and burner

$\searrow$ High system availability

$\searrow$ Very low emission levels and quick ROI thanks to outstanding efficiency

$\searrow$ Designed for all standard and special liquid and gas fuels such as biogas

$\searrow$ Turbine and burner can be operated independently

$\searrow$ Increased production flexibility and independence of energy cost fluctuations

\section{Maximum burner capacity in MW}

\begin{tabular}{|c|c|c|c|}
\hline \multirow[t]{2}{*}{ Size } & \multirow{2}{*}{$\begin{array}{c}\text { Maximum } \\
\text { power }\end{array}$} & \multicolumn{2}{|c|}{ Maximum control range } \\
\hline & & Oil operation & Gas operation \\
\hline 2 & 2 & $1: 2 / 1,0 \mathrm{MW}$ & $1: 4 / 0,5 \mathrm{MW}$ \\
\hline 5 & 5 & $1: 3 / 1,7 \mathrm{MW}$ & $1: 5 / 1,0 \mathrm{MW}$ \\
\hline 8 & 8 & $1: 3 / 2,7 \mathrm{MW}$ & $1: 6 / 1,3 \mathrm{MW}$ \\
\hline 13 & 13 & $1: 3 / 4,3 \mathrm{MW}$ & $1: 6 / 2,2 \mathrm{MW}$ \\
\hline 20 & 20 & $1: 3 / 6,7 \mathrm{MW}$ & $1: 6 / 3,3 \mathrm{MW}$ \\
\hline
\end{tabular}

Emission levels*

\begin{tabular}{cc}
\hline Natural gas & Light oil"* \\
\hline $80-100 \mathrm{NO}_{x}\left[\mathrm{mg} / \mathrm{m}^{3}\right]$ & $130-180 \mathrm{NO}_{\mathrm{x}}\left[\mathrm{mg} / \mathrm{m}^{3}\right]$ \\
(with flue gas recirculation) & (with flue gas recirculation)
\end{tabular}




\section{Anexo D}

Las aportaciones de esta tesis fueron expuestas en dos trabajos, uno en la participación del congreso IEEE CONCAPAN 2018 XXXVIII Convención de Centroamérica y Panamá, que se llevó a cabo en San Salvador, El Salvador en el centro de convenciones del Hotel Crowne Plaza San Salvador del 7 al 9 de noviembre de 2018. El tema en torno al cual giraba el congreso era "Ingeniería al Servicio del Desarrollo Sostenible". Se participó en la modalidad de conferencista, con el trabajo titulado "Gas turbine for Micro-CHP system and heat recovery with a conventional steam boiler: Thermodynamic analysis", cuyos autores son: Adriana Santamaria Padilla y Hernando Romero Paredes Rubio.

El trabajo está publicado en la revista IEEE con el DOI: 10.1109/CONCAPAN.2018.8596672. Electronic ISBN: 978-1-5386-6122-2, USB ISBN: 978-1-5386-6121-5, Print on Demand(PoD) ISBN: 978-1-5386-6123-9.

El Segundo trabajo fue expuesto en World Sustainable Energy Days bajo el lema Clean. Competitive. Connected. En Wels, Austria del 27 de Febrero al 1 de marzo de 2019. Se participó en el Young Energy Researchers Conference- Energy Efficiency, con el trabajo titulado: "Heat recovery with steam boiler of waste heat from MGT: Thermodynamic analysis", cuyos autores son: Adriana Santamaria Padilla y Hernando Romero Paredes Rubio. 


\section{IEEE}

El Comité Organizador de la Trigésima Octava Convención de Ingeniería Eléctrica y Electrónica de Centroamérica y Panamá

Otorga el presente Diploma a:

\section{ADRIANA SANTAMARIA}

Por su valiosa participación como PONENTE en la CONCAPAN XXXVIII, El Salvador 2018 con la charla técnica

Gas turbine for Micro-CHP system and heat recovery with a conventional steam

boiler: Thermodynamic analysis

Dado en San Salvador, el 8 de noviembre de 2018
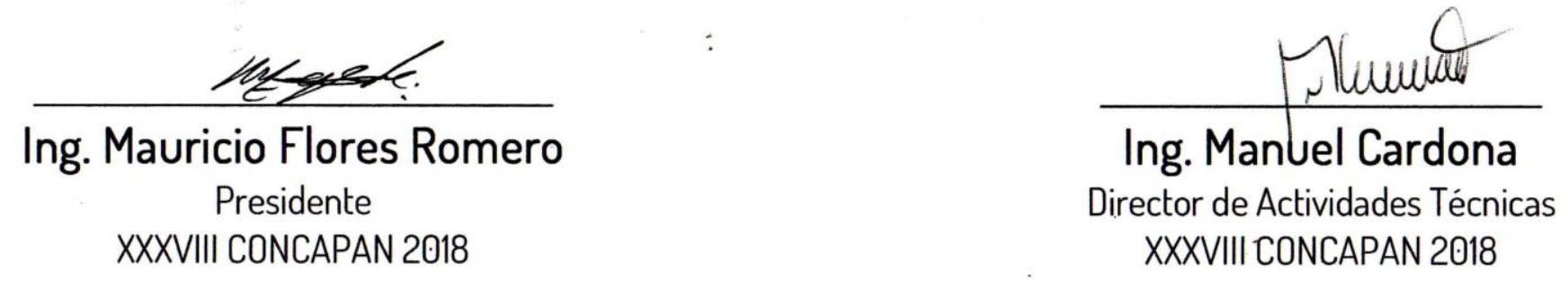


\section{Gas turbine for Micro-CHP system and heat recovery with a conventional steam boiler: Thermodynamic analysis}

\author{
$1^{\text {st }}$ Adriana Santamaria Padilla \\ Posgrado en Energía y Medio Ambiente \\ Universidad Autónoma Metropolitana-Iztapalapa \\ Ciudad de México, México \\ aspenergia@xanum.uam.mx
}

\author{
$2^{\text {nd }}$ Hernando Romero Paredes R. \\ Departamento de Ingenieria de Procesos e Hidraulica \\ Universidad Autónoma Metropolitana-Iztapalapa \\ Ciudad de México, México \\ hrp@xanum.uam.mx
}

\begin{abstract}
The main objective of this paper is to demonstrate the advantages of using the micro gas turbine (MGT) exhaust gasses to substitute the air needed in the combustion camera, conventional steam boiler. It was evaluated the performance of the Capstone C30 gas turbine. Because the exhaust gasses from de MGT comes with a high air excess they can be used to feed the air needed in a conventional steam boiler. In this work was evaluated the effect that has the use of those gasses on the heat convection transfer coefficient in order to know the advantage or disadvantage using those gasses. A thermodynamic analysis model was developed to know the behavior of a conventional steam boiler when the exhaust gasses are used as air for the combustion process. It was gotten the operation parameters of the MGT which are: pressure ratio $=3.2$ and the temperature turbine inlet $=1050^{\circ} \mathrm{C}$. Derating coefficient of this turbine was calculated and it was estimated that for each 500 meters above sea level the generated power decrease $5.5 \%$ and when the temperature increase $5^{\circ} \mathrm{C}$ power decrease $3.9 \%$. Meanwhile, when the exhaust gasses of the MGT are feeded to the steam boiler it could save about of $10 \%$ of fuel. Because MGT exhaust gasses are relatively poor in oxygen the mass flow feeded to the steam boiler is increased and then the flow regime is also increased. Whit these phenomena the convection heat transfer coefficient is higher than the conventional ones. In this work was found that this coefficient increased more than $12 \%$ respect to the original system.
\end{abstract}

Keywords-Micro-CHP; derating coeffcient; steam boiler; heat recovery exhaust gasses; convective heat transfer coefficient

\section{INTRODUCCIÓN}

Uno de los grandes problemas de la industria nacional es la ineficiencia en el uso de la energía. Existen muchas acciones que se puede emprender para reducir los consumos de energía en las empresas industriales, comerciales y de servicios. Todas esas acciones requieren de un análisis preciso, una evaluación técnica y económica para determinar su viabilidad técnica y económica. De acuerdo con la CONUEE (2009) algunas de las tecnologías que se están utilizando para reducir los consumos de energía son el ciclo combinado, la cogeneración y la micro-cogeneración, donde esta última se ha desarrollado muy poco en México [1].
En el artículo 36, fracción II, de la Ley de la Industria Eléctrica y en su Reglamento derivado, artículo 103 puntualiza que se entiende por cogeneración a la:

I. Producción de energía eléctrica conjuntamente con vapor u otro tipo de energía térmica secundaria, o ambas.

II. Producción directa o indirecta de energía eléctrica a partir de energía térmica no aprovechada en los procesos de que se trate

III. Producción directa o indirecta de energía eléctrica utilizando combustibles producidos en los procesos de que se trate.

El consumo de energía térmica en México representa alrededor de $77 \%$ del consumo energético total, el aprovechamiento del calor residual sólo se emplea parcialmente en algunas industrias. El rendimiento de las instalaciones de cogeneración está alrededor del 80\% [2]; razón por la que actualmente se están fomentando este tipo de instalaciones.

En la Fig. 1 se muestra la evolución histórica de la capacidad instalada de cogeneración en México por la Comisión Reguladora de Energía (CRE) que tiene registrados en el período de 1994 a 2017 [3]. Se observa que a partir del año 2015 se instalaron un gran número de sistemas de cogeneración, debido a la reforma energética en México.

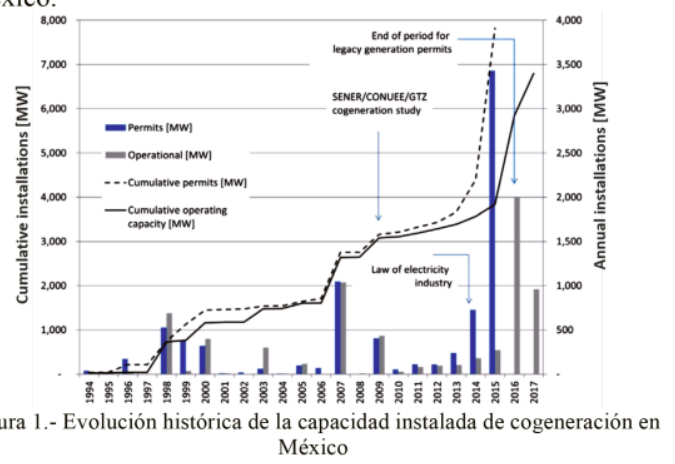

La cogeneración al producir conjuntamente calor y electricidad en el centro de consumo hace posible utilizar los 
gases efluentes de una micro-turbina de gas (MTG) para diferentes aplicaciones, algunas de ellas son: el calentamiento de agua, producción de vapor, refrigeración por absorción, calefacción, entre otras [4]. Las industrias que tienen el mayor potencial de mercado estimado para el uso de MTG en CHP son: alimentos, bebidas, pulpa y papel, textiles, hospitales, hoteles y edificios institucionales.

Los sistemas de micro-cogeneración (MCHP) generalmente implican un motor principal, los cuales pueden ser: un motor Stirling, motores alternativos, MTG, ciclo Rankine y celdas de combustible [5]. Existe un interés particular en el empleo potencial de tecnologías como MTG y motores alternativos.

En este trabajo se analiza la factibilidad de emplear una MTG y acoplar sus gases de escape a una caldera convencional para sustituir el aire de combustión y reducir el consumo energético. Se hace la evaluación con una MTG Capstone C30 y una caldera de 60 BHP Cleaver Brooks. El objetivo es determinar los posibles efectos que se puedan tener en la operación de la caldera al suministrarle los gases calientes efluentes de una MTG.

\section{NOMENCLATURA}

\begin{tabular}{|c|c|c|c|}
\hline Cp & $\begin{array}{l}\text { calor específico; } \\
{[\mathrm{kJ} / \mathrm{kgK}]}\end{array}$ & disp & disponible, \\
\hline CTU & $\begin{array}{l}\text { consumo térmico } \\
\text { unitario; }[\mathrm{kJ} / \mathrm{kWh}] \text {, }\end{array}$ & elec & eléctrica, \\
\hline D & diámetro; $[\mathrm{m}]$ & gc & $\begin{array}{l}\text { gases de } \\
\text { combustión, }\end{array}$ \\
\hline Ec & $\begin{array}{l}\text { energía de combustible; } \\
{[\mathrm{kW}]}\end{array}$ & int & interior, \\
\hline $\mathrm{h}$ & $\begin{array}{l}\text { coeficiente convectivo; } \\
{\left[\mathrm{W} / \mathrm{m}^{2}{ }^{\circ} \mathrm{C}\right]}\end{array}$ & loss & pérdidas, \\
\hline $\mathrm{k}$ & $\begin{array}{l}\text { Conductividad térmica; } \\
{\left[\mathrm{W} / \mathrm{m}^{\circ} \mathrm{C}\right]}\end{array}$ & $\mathrm{m}$ & motor, \\
\hline m & Flujo másico; $[\mathrm{kg} / \mathrm{s}]$, & mec & mecánica, \\
\hline $\mathrm{Nt}$ & número de tubos, & Nom & Nominal, \\
\hline $\mathrm{Nu}$ & Nusselt, & real & \\
\hline $\mathrm{P}$ & presión; [bar], & sic & $\begin{array}{l}\text { isoentrópico del } \\
\text { compresor }\end{array}$ \\
\hline $\operatorname{Pr}$ & Prandtl, & sit & $\begin{array}{l}\text { isoentrópico de la } \\
\text { turbina }\end{array}$ \\
\hline Q & calor; $[\mathrm{kW}]$ & st & estequiometrica, \\
\hline Qc & & sum & suministrado, \\
\hline $\mathrm{R}$ & $\begin{array}{l}\text { constante de los gases } \\
\text { ideales; }[\mathrm{kJ} / \mathrm{kmolK}] \text {, }\end{array}$ & $\mathrm{T}$ & turbina, \\
\hline $\operatorname{Re}$ & $\begin{array}{l}\text { Reynolds, } \\
\text { relación aire }\end{array}$ & tu & tubos, \\
\hline RAC & $\begin{array}{l}\text { combustible; } \\
{\left[\mathrm{kg}_{\text {aire }} / \mathrm{kg}_{\text {comb }}\right]}\end{array}$ & th & térmica, \\
\hline RPM & $\begin{array}{l}\text { revoluciones por } \\
\text { minuto; [min] }\end{array}$ & $\mathrm{u}$ & universal, \\
\hline $\mathrm{T}$ & $\begin{array}{l}\text { temperatura; }\left[{ }^{\circ} \mathrm{C}\right], \\
\text { coeficiente global de }\end{array}$ & vap & vapor \\
\hline $\mathrm{U}$ & $\begin{array}{l}\text { transferencia de calor; } \\
{\left[\mathrm{W} / \mathrm{m}^{2 \circ} \mathrm{C}\right]}\end{array}$ & Letras & griegas \\
\hline $\mathrm{V}$ & $\begin{array}{l}\text { volumen especifico; } \\
{\left[\mathrm{m}^{3} / \mathrm{kg}\right]}\end{array}$ & $\pi$ & $\begin{array}{l}\text { relación de } \\
\text { compresión, }\end{array}$ \\
\hline W & $\begin{array}{l}\text { trabajo por unidad de } \\
\text { masa; }[\mathrm{kJ} / \mathrm{kg}]\end{array}$ & $\eta$ & eficiencia; [\%], \\
\hline$\dot{W}$ & potencia; $[\mathrm{MW}, \mathrm{kW}]$ & $\rho$ & densidad; $\left[\mathrm{kg} / \mathrm{m}^{3}\right]$ \\
\hline $\mathrm{x}$ & exceso de aire; [\%], & \multicolumn{2}{|c|}{ Abreviaturas } \\
\hline Sub indices & & $\mathrm{CC}$ & $\begin{array}{l}\text { Cámara de } \\
\text { combustion, }\end{array}$ \\
\hline
\end{tabular}

\begin{tabular}{|c|c|c|c|}
\hline $\mathrm{amb}$ & ambiente, & CHP & $\begin{array}{l}\text { combined heat and } \\
\text { power, }\end{array}$ \\
\hline aire & aire, & GE & $\begin{array}{l}\text { generador } \\
\text { eléctrico, }\end{array}$ \\
\hline & caldera, & GEI & $\begin{array}{l}\text { gases de efecto } \\
\text { invernadero, }\end{array}$ \\
\hline omb & combustible, & GN & gas natural, \\
\hline & combustible-aire, & MM & masa molecular, \\
\hline mbtotal & combustible total, & PCI & $\begin{array}{l}\text { poder calorifico } \\
\text { inferior. }\end{array}$ \\
\hline
\end{tabular}

\section{METODOLOGÍA}

\section{A. Turbina de gas}

Los gases de combustión de una MTG salen a altas temperaturas, alrededor de los 270 a $320^{\circ} \mathrm{C}$, dependiendo de la potencia de esta, los cuales en sistemas convencionales son mandados directamente a la atmósfera. Razón por la cual es importante realizar un análisis de factibilidad de la implementación de un sistema continuo de aprovechamiento de los gases de combustión de la MTG en una caldera convencional que evitara la inversión de un recuperador de calor.

En la Tabla 1 se muestra las especificaciones de la MTG de estudio proporcionados por el fabricante [6].

TABLA 1.- Especificaciones de la MTG de estudio.
\begin{tabular}{|l|c|}
\hline Modelo & C30 \\
\hline Potencia nominal de salida $[\mathrm{kW}]$ & 30 \\
\hline Rendimiento eléctrico $[\%]$ & 26 \\
\hline Presión de entrada del comb $[\mathrm{bar}]$ & 3.79 a 4.14 \\
\hline Flujo de combustible $\mathrm{HHV}[\mathrm{MJ} / \mathrm{h}]$ & 444 \\
\hline $\mathrm{CTU}[\mathrm{kJ} / \mathrm{kWh}]$ & 13800 \\
\hline NOx $@ 15 \% \mathrm{O}_{2}$ ppmvd & 9 \\
\hline Caudal de gases de escape $[\mathrm{kg} / \mathrm{s}]$ & 0.31 \\
\hline Temperatura salida de gases[ $\left[{ }^{\circ} \mathrm{C}\right]$ & 275 \\
\hline Calor disponible a $70^{\circ} \mathrm{C}[\mathrm{kW}]$ & 65 \\
\hline
\end{tabular}

El análisis se realizará usando como combustible gas natural, con la composición mostrada en la Tabla 2, obteniéndose un $\mathrm{PCI}=38,563 \mathrm{~kJ} / \mathrm{m}^{3}[7]$.

TABLA 2.- Composición del gas natural
\begin{tabular}{|c|c|}
\hline Compuesto & Porcentaje [\%] \\
\hline $\mathrm{CH}_{4}$ & 94 \\
\hline $\mathrm{C}_{2} \mathrm{H}_{6}$ & 3 \\
\hline $\mathrm{CO}_{2}$ & 1 \\
\hline $\mathrm{N}_{2}$ & 1 \\
\hline $\mathrm{C}_{3} \mathrm{H}_{8}$ & 0.5 \\
\hline $\mathrm{C}_{4} \mathrm{H}_{10}$ & 0.3 \\
\hline $\mathrm{C}_{5} \mathrm{H}_{12}$ & 0.2 \\
\hline
\end{tabular}

Se determinan las condiciones de operación a partir de los datos del fabricante de la MTG bajo condiciones ISO. El flujo de combustible se calcula usando (2).

$$
\begin{aligned}
& E_{c}=\dot{m}_{\text {comb }} P C I \\
& \dot{m}_{c o m b}=\frac{E_{c}}{P C I}
\end{aligned}
$$

Una vez obtenido éste, se determina el flujo de aire a partir del caudal de los gases de escape:

$$
\dot{m}_{\text {aire }}=\dot{m}_{g c}-\dot{m}_{\text {comb }}
$$

Conocido ambos flujos se calcula la $R A C_{\text {real }}$ de acuerdo $\operatorname{con}(4)$. 


$$
R A C_{\text {real }}=\frac{\dot{m}_{\text {aire }}}{\dot{m}_{\text {comb }}}
$$

Para obtener la $R A C_{s t}$ se utiliza la ecuación de combustión (5) con base a la composición del gas natural mostrada en la Tabla 2.

$$
\mathrm{C}_{\mathrm{H} H m}+(1+x)\left(n+\frac{m}{4}\right)\left(\mathrm{O}_{2}+3.76 \mathrm{~N}_{2}\right) \rightarrow \mathrm{nCO}_{2}+\frac{m}{2} \mathrm{H}_{2} \mathrm{O}+x\left(n+\frac{m}{4}\right) \mathrm{O}_{2}+(1+x)\left(n+\frac{m}{4}\right) 3.76 \mathrm{~N}_{2}(5)
$$

Bajo estas condiciones se obtiene la forma compacta siguiente:

$$
C_{1.037} H_{4.034}
$$

Cuya masa molecular es:

$$
M M=12 n+m
$$

Una vez conocido lo anterior, se calcula la $R A C_{s t}$ usando (8)

$$
R A C_{s t}=\frac{\left(n+\frac{m}{4}\right) * 4.76 * 29}{M M}
$$

La $R A C_{\text {real }}$ se calcula de la siguiente manera:

$$
R A C_{\text {real }}=(1+x)\left(R A C_{s t}\right)
$$

De la ecuación (9) se obtiene el exceso de aire:

$$
x=\frac{R A C_{\text {real }}}{R A C_{s t}}-1
$$

La Fig. 2 muestra el diagrama esquemático de operación de la MTG. Se observa que el aire medioambiental es succionado por el compresor y comprimido hasta una presión dada. Éste entra a un recuperador de calor para después entrar a la cámara de combustión donde se mezcla con el combustible utilizado y se efectúa la combustión. Estos gases se expanden en la turbina de gas produciendo un trabajo y finalmente pasan por el recuperador. Una parte del trabajo obtenido se emplea la operación del compresor y la diferencia es convertida en energía eléctrica por medio del generador [8].

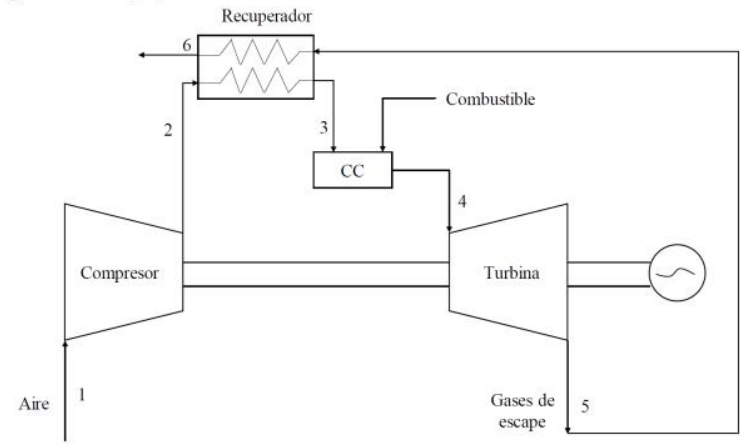

Figura 2.- Diagrama esquemático de una MTG

Las propiedades termodinámicas de las MTG de estudio se determinan de la siguiente manera:

Estado 1.- Admisión de aire al compresor. Se toma como estado de referencia las siguientes condiciones atmosféricas: $\mathrm{T}=15{ }^{\circ} \mathrm{C}$ y $\mathrm{P}=1.013$ bar. Con esos valores y utilizando la ecuación de gas ideal se obtiene el volumen específico considerando una mol de aire.

$$
v=\frac{R_{\text {aire }} T}{P}
$$

Para calcular el volumen específico del aire de la MTG se utiliza el flujo de aire a condiciones ISO, el cual se mantendrá constante en todas las variaciones del presente análisis puesto que la turbina está diseñada para ese volumen específico sin importar las condiciones atmosféricas. Sin embargo, sí cambia el flujo másico:

$$
\dot{V}_{\text {aire }}=v_{1} \dot{m}_{\text {airelSo }}
$$

Estado 2.- Salida del aire del compresor. El fabricante no proporciona la $\pi$, por tal razón se tiene que iterar. Conforme a lo encontrado en la literatura ésta puede operar en un intervalo de $\pi=1.5$ a $7 ; \pi$ se varió en ese intervalo $[9,10]$. Una vez obtenida $\pi$ se obtiene $P_{2}$ y a partir de la eficiencia del compresor (se considera en primera instancia de 0.8 ) se obtiene $\mathrm{T}_{2}$ :

$$
T_{2}=T_{1}\left[1+\frac{1}{\eta_{\text {sic }}}\left(\pi^{x}-1\right)\right]
$$

Una vez conocido $T_{2}$ y $P_{2}$ se puede obtener el volumen específico $\mathrm{V}_{2}$ usando (11).

Estado 4.- Entrada de los gases de combustión a la turbina. El fabricante no proporciona la temperatura a la entrada de los gases. Para obtener esta temperatura se buscó en la literatura el intervalo de temperaturas en el cual operan estas turbinas y acotar las iteraciones, encontrando un intervalo que va de 700 a $1300^{\circ} \mathrm{C}[11,12,13]$. Además, considerando que no hay caídas de presión en el regenerador y en la cámara de combustión, $\mathrm{P}_{4}=\mathrm{P}_{2}$, conocidas $\mathrm{T}_{4}$ y $\mathrm{P}_{4}$ usando (11) se obtiene $\mathrm{v}_{4}$.

Estado 5s.- Salida de la turbina isoentrópico. Considerando la expansión isoentrópica de la turbina se obtiene $\mathrm{T}_{5 \mathrm{~s}}$ con la siguiente ecuación:

$$
T_{5 s}=T_{4}\left(\frac{1}{\pi^{x}}\right)
$$

Estado 5.- Salida de la turbina real. A partir de la eficiencia de la turbina se obtiene $\mathrm{T}_{5}$ :

$$
\eta_{\text {sit }}=\frac{T_{4}-T_{5}}{T_{4}-T_{5 s}}
$$

Estado 3.- Entrada a la cámara de combustión. La temperatura del aire a la entrada de la cámara de combustión se obtiene a partir de la definición de la eficacia del regenerador:

$$
E=\frac{T_{3}-T_{2}}{T_{5}-T_{2}}
$$

Estado 6.- Salida del regenerador de la corriente caliente. La temperatura $T_{6}$, que descargan el regenerador a proceso, se obtiene a partir de un abalance de materia y energía como se muestra a continuación:

$$
\dot{m} C p\left(T_{5}-T_{2}\right)=\dot{m} C p\left(T_{5}-T_{6}\right)
$$

También se puede obtener a partir de la eficacia del regenerador:

$$
T_{6}=T_{5}-E\left(T_{5}-T_{2}\right)
$$

Una vez conocidos los estados termodinámicos se puede calcular el calor disponible de la MTG:

$$
Q_{d i s p}=\dot{m}_{g c} C p_{g c}\left(T_{5}-T_{r e q}\right)
$$

Se sabe que, para generar potencia mecánica a partir de la combustión de una mezcla de combustible y aire en determinadas condiciones de presión y temperatura las condiciones ambientales juegan un papel importante $[9,14]$. El coeficiente de derrateo se calcula variando la presión 
como función de la altura SNM (de 0 a $3500 \mathrm{msnm}$, con intervalos de $500 \mathrm{~m}$ ) y la temperatura $\left(\right.$ de 5 a $50^{\circ} \mathrm{C}$, con intervalos de $5^{\circ} \mathrm{C}$ ) [15].

\section{B. Caldera}

Las calderas son equipos diseñados para transferir calor producido por combustión, o mediante electricidad, a un fluido determinado, genera y entrega vapor en la cantidad y calidad requeridas por el usuario final [16]

Las calderas pirotubulares (tubos de humo) son las más empleadas en la mayor parte de las plantas industriales, donde los gases calientes fluyen por el interior de los tubos que son sumergidos en agua dentro de un casco como se muestra en la Fig. 3. Las presiones operativas de diseño máximas son a 15 bar. La capacidad varía entre 10 y 800 BHP, equivalentes a producciones de vapor de 160 a 12,500 $\mathrm{kg} / \mathrm{h}[17]$.

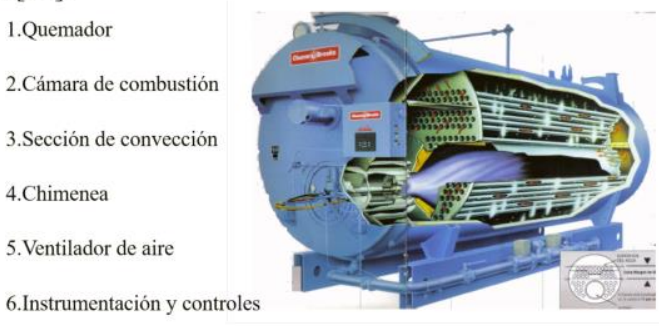

Figura 3.- Caldera pirotubular (tubos de humo) [18].

En la Fig. 4 se muestra el diagrama esquemático de la caldera de estudio de 60 BHP, 4 pasos Dry-Back. El objetivo de este trabajo es sustituir el flujo de aire por los gases provenientes de una MTG y así aprovechar el calor de esos gases para reducir el consumo de combustible. Debido a que esos gases vienen con un elevado exceso de aire en principio no perturban el proceso de combustión convencional de la caldera. Bajo esta premisa se reduce el consumo de combustible. Se debe por lo tanto conocer la magnitud de la perturbación en los coeficientes de transferencia de calor en el as de tubos. En la Tabla 5 se muestran las especificaciones de la caldera de 60 BHP. Para determinar el coeficiente convectivo de transferencia de calor del lado de los gases de combustión se analizan las condiciones de operación de la caldera bajo condiciones estándar $[19,20]$ :

TABLA 3.- Especificaciones caldera Cleaver Brooks de 4 pasos Dry-Back
\begin{tabular}{|l|c|}
\hline BHP & $\mathbf{6 0}$ \\
\hline Producción de vapor $[\mathrm{kg} / \mathrm{s}]$ desde $\mathrm{Y}$ hasta $100^{\circ} \mathrm{C}$ & 0.26082 \\
\hline Potencia de la caldera $[\mathrm{kW}]$ & 938.94 \\
\hline Superficie de calentamiento (Fireside) $\left[\mathrm{m}^{2}\right]$ & 27.87 \\
\hline Flujo de combustible $[\mathrm{kg} / \mathrm{s}]$ & 0.01463 \\
\hline Diámetro del tubo $[\mathrm{m}]$ & 0.0381 \\
\hline Espesor del tubo $[\mathrm{m}]$ & 0.00241 \\
\hline Longitud de los tubos $[\mathrm{m}]$ & 2.971 \\
\hline Conductividad térmica del acero al carbón $\left[\mathrm{W} / \mathrm{m}^{\circ} \mathrm{C}\right]$ & 29 \\
\hline Numero de tubos en la caldera & 74 \\
\hline
\end{tabular}

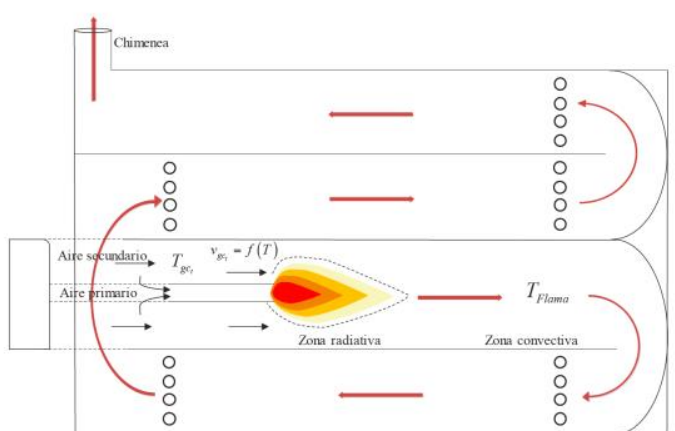

Figura 4.- Diagrama esquemático de una caldera de 4 pasos Dry-Back

1. A partir de los datos del fabricante se obtiene la cantidad de vapor estándar disponible mediante:

$$
\dot{Q}_{\text {vap }}=\dot{m}_{\text {vapNom }} \Delta h
$$

Donde ese $\Delta h$ está en función de las entalpias de condiciones de operación y ambientales, por lo tanto, al ser definidas para operar bajo nuevas condiciones se recalcula el flujo de vapor real con la siguiente ecuación:

$$
\dot{m}_{\text {vap Real }}=\frac{\dot{m}_{\text {vapNom }}}{f e}
$$

Donde: fe es el factor de evaporación.

2. Conocido el flujo de vapor real, se puede calcular el calor suministrado por el combustible:

$$
\dot{Q}_{\text {sum }}=\frac{\dot{Q}_{v a p}}{\eta}
$$

3. Se calcula el flujo de combustible:

$$
\dot{m}_{\text {comb }}=\frac{\dot{Q}_{\text {sum }}}{P C I}
$$

4. Posteriormente el flujo de gases de combustión en función del exceso de aire, el cual varia de $0-25 \%$, se determina usando (24).

$$
\dot{m}_{g c}=\left(R A C_{\text {real }}+1\right) \dot{m}_{\text {comb }}
$$

5. Se obtiene el flujo de aire de la caldera de forma estequiométrica $(\mathrm{x}=0)$ y con exceso de aire $(\mathrm{x} \neq 0)$.

$$
\dot{m}_{\text {aire }}=\dot{m}_{g c}-\dot{m}_{\text {comb }}
$$

Conocidos estos flujos se puede calcular el coeficiente convectivo de los gases de combustión [21]:

- Usando (24), se obtiene el flujo de gases de combustión para cada tubo:

$$
\dot{m}_{g c} / t u=\frac{\dot{m}_{g c}}{N_{T}}
$$

- Se determina la velocidad de los gases por tubo:

$$
v_{g c, t u}=\frac{\dot{V}_{g c} / t u}{\rho_{g c\left(\bar{I}_{g c}\right.} A_{t u}}
$$

Donde la densidad de los gases de combustión está en función de una temperatura promedio que llevan éstos según:

$$
\bar{T}_{g c}=\frac{T_{\text {Flama }}-T_{p r o p, g c}}{2}
$$


- Conocida la velocidad de los gases de combustión se determina el Reynolds:

$$
\operatorname{Re}=\frac{v_{t u} d_{\text {int }}}{v}
$$

A partir del número de Reynolds se emplean correlaciones del número de Nusselt dependiendo del tipo de flujo obtenido (laminar, transitorio o turbulento). Con el número de Nusselt se obtiene el coeficiente convectivo buscado.

$$
h_{1}=\frac{N u k}{D}
$$

Donde el Nusselt se determina en función del régimen de flujo [21]:

Flujo turbulento: $N u=0.22 \operatorname{Re}^{0.8} \operatorname{Pr}^{0.5}$

$$
\text { Flujo en transición: } N u=\frac{(f / 2)(\mathrm{Re}-1000) \operatorname{Pr}}{1+12.7 \sqrt{f / 2}\left(\operatorname{Pr}^{2 / 3}-1\right)}
$$

Donde: $f=(1.58 \ln \operatorname{Re}-3.28)^{-2}$

\section{Acoplamiento gases de combustión MTG-Caldera}

Para hacer el análisis del acoplamiento de los gases efluentes de una MTG a una caldera se necesita conocer su composición. Se obtiene usando (5) para cuando esta reacción se lleva a cabo con una cierta cantidad de kilo moles $\left(\mathrm{Na}_{T}\right)$ de combustible y un cierto exceso de aire. Con los datos de las MTG se conoce la cantidad de combustible con la que se alimentan y se puede calcular el número de $\mathrm{Na}_{T}$ alimentados. Usando (7) se conoce la masa molecular del combustible y se determina el número de $N a_{T}$ :

$$
N a_{T}=\frac{\dot{m}_{c o m b T}}{M M_{c}}
$$

La ecuación (5) se puede reescribir para determinar la composición de los gases de escape:

$$
\begin{aligned}
& N a_{T} C_{n} H_{n}+\left(1+x_{T}\right)\left[N a_{T}\left(n+\frac{m}{4}\right)\left(O_{2}+3.76 N_{2}\right)\right] \rightarrow N a_{T} n C O_{2}+ \\
& N a_{T} \frac{m}{2} H_{2} O+x_{T} N a_{T}\left(n+\frac{m}{4}\right) O_{2}+\left(1+x_{T}\right)\left[N a_{T}\left(n+\frac{m}{4}\right)\right] 3.76 N_{2}
\end{aligned}
$$

Conocida la composición de los gases de escape y el flujo de los mismos se puede calcular el calor disponible usando (19) y considerando el valor de $\mathrm{T}_{\text {req }}=25^{\circ} \mathrm{C}$. Con la cantidad de calor por los gases de combustión y el calor requerido de la caldera usando (22) se calcula el nuevo flujo de combustible que requiere la caldera al introducir los gases de combustión a la misma con:

$$
\begin{aligned}
& Q_{\text {comb2 } 2}=Q_{s i m}-Q_{g c T} \\
& \dot{m}_{\text {comb } 2}=\frac{Q_{\text {comb2 }}}{P C I}
\end{aligned}
$$

Con este flujo de combustible de la caldera se calcula la cantidad de oxigeno que requiere de forma estequiométrica usando (37).

$$
N a_{C} C_{n} H_{m}+\left[N a_{C}\left(n+\frac{m}{4}\right)\right]\left(O_{2}+3.76 N_{2}\right) \rightarrow N_{c} n C O_{2}+N a_{c} \frac{m}{2} H_{2} O+\left[N a_{C}\left(n+\frac{m}{4}\right)\right] 3.76 N_{2}
$$

Empleando la composición de los gases provenientes de la MTG se realiza el balance de masa de la combustión, de suerte de conocer si existe exceso o deficiencia de oxigeno con esos gases:

$$
\begin{aligned}
& N a_{C} C_{n} H_{m}+N a_{T} n C_{2}+N a_{T} \frac{m}{2} H_{2} O+x_{T} N a_{T}\left(n+\frac{m}{4}\right) O_{2}+\left(1+x_{T}\right)\left[\mathrm{Na}_{C}\left(n+\frac{m}{4}\right)\right] 3.76 N_{2} \\
& \rightarrow \mathrm{Na}_{T} n \mathrm{CO}_{2}+\mathrm{Na}_{C} n \mathrm{CO}_{2}+\mathrm{Na}_{T} \frac{m}{2} \mathrm{H}_{2} \mathrm{O}+\mathrm{Na} a_{C} \frac{m}{2} \mathrm{H}_{2} \mathrm{O}+\alpha O_{2}+\left(1+x_{T}\right)\left[\mathrm{Na}\left(n+\frac{m}{4}\right)\right] 3.76 \mathrm{~N}_{2}
\end{aligned}
$$

Donde: $\quad \alpha<0$ se tiene déficit de oxigeno

$$
\begin{aligned}
& \alpha=0 \text { es estequiométrico } \\
& \alpha>0 \text { se tiene exceso de oxigeno }
\end{aligned}
$$

Tomando un valor de $\alpha<0$ la ecuación de combustión queda:

$$
\begin{aligned}
& \mathrm{Na}_{C} \mathrm{C}_{n} \mathrm{H}_{w}+\mathrm{Na}_{T} n \mathrm{CO}_{2}+\mathrm{Na}_{T} \frac{m}{2} \mathrm{H}_{2} \mathrm{O}+x_{T} \mathrm{Na}_{T}\left(n+\frac{m}{4}\right) \mathrm{O}_{2}+\left(1+x_{T}\right)\left[\mathrm{Na}_{C}\left(n+\frac{m}{4}\right)\right] 3.76 \mathrm{~N}_{2} \\
& +\left(1+x_{C}\right)\left[\alpha\left(\mathrm{O}_{2}+3.76 \mathrm{~N}_{2}\right)\right] \rightarrow \mathrm{Na}_{T} n \mathrm{CO}_{2}+\mathrm{Na}_{C} n \mathrm{CO}_{2}+\mathrm{Na}_{T} \frac{m}{2} \mathrm{H}_{2} \mathrm{O}+\mathrm{Na}_{C} \frac{m}{2} \mathrm{H}_{2} \mathrm{O}+x_{C} \alpha \mathrm{O}_{2} \\
& +\left(1+x_{T}\right)\left[\mathrm{Na}_{T}\left(n+\frac{m}{4}\right)\right] 3.76 \mathrm{~N}_{2}+\left(1+x_{C}\right) \alpha 3.76 \mathrm{~N}_{2}
\end{aligned}
$$

La ecuación (39) se emplea para determinar el flujo de gases de combustión al variar el exceso de aire. Con estos flujos se determina el número de Reynolds, número de Nusselt y el coeficiente convectivo de transferencia de calor del lado de los gases.

\section{RESULTADOS}

\section{A. Turbina de gas}

Como se desconocen $\pi$ y la temperatura de entrada de los gases a la turbina (TET), se realizó la iteración de 3 variables: trabajo motor, TET y la relación de presiones, como se muestra en la Fig. 5. De esta evaluación se obtuvo que la MTG C30 opera con una $\pi=3.2$ y TET $=1050{ }^{\circ} \mathrm{C}$. A esas condiciones se obtiene la potencia reportada por el fabricante y el calor disponible para proceso reportado en la literatura $[22,23]$.

Conocidos $\pi$ y TET, se obtiene el coeficiente de derrateo (Fig.6). Se observa que el comportamiento de la MTG C30 variando las condiciones atmosféricas $(T, P)$, tiene un decremento en la potencia del $5.5 \%$ por cada $500 \mathrm{msnm}$ y del $3.9 \%$ por cada $5^{\circ} \mathrm{C}$ que aumenta la temperatura.

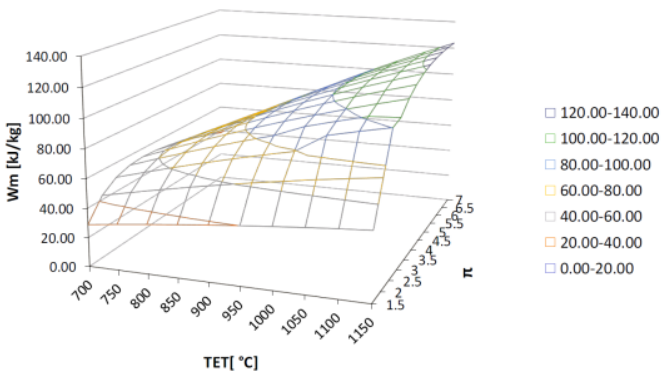

Figura 5.- Variación de parámetros de la MTG Capstone C30. 


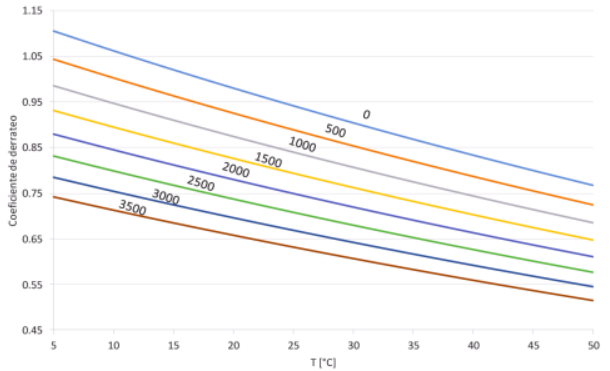

Figura 6.- Derrateo de la MTG Capstone C30.

\section{B. Acoplamiento de gases de combustión en la caldera}

Una vez que se conoce la variación de flujo de gases en función del exceso de aire en la caldera operando de manera convencional o empleando los gases provenientes de la MTG (Fig. 7), se puede observar que el flujo de gases provenientes de la MTG es mayor al empleado convencionalmente al empleado por la caldera. Esta situación puede ser negativa si el régimen de operación se conserva laminar porque el coeficiente de transferencia de calor, en el caso limite, permanecería constante y por consiguiente la temperatura de los gases en la chimenea de la caldera se incrementaría. Para corroborar este proceso, se evalúa el coeficiente convectivo de transferencia de calor para ambos casos (Fig. 8). Se observa que el coeficiente convectivo de transferencia de calor es mayor cuando se suministran los gases efluentes de la MTG. No obstante, la diferencia se va reduciendo en la medida que se incrementa el exceso de aire. Es de notarse que una caldera que opera con gas natural difícilmente llegará a tener un exceso de aire mayor al $20 \%$. De esta manera se demuestra la conveniencia del empleo de los gases de combustión de la MTG en una caldera convencional.

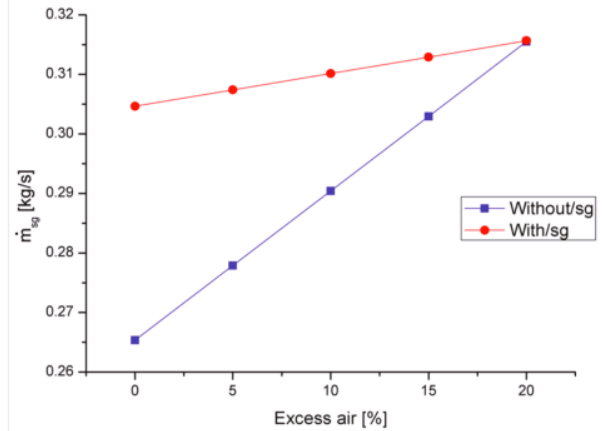

Figura 7.- Flujo de gases de combustión en función del exceso de aire

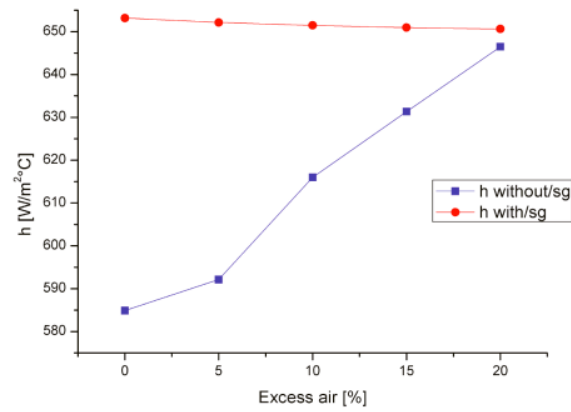

Figura 8.- Coeficiente convectivo de transferencia de calor en función del exceso de aire

\section{CONCLUSIONES}

En este trabajo se encontró que las condiciones de operación de la MTG de Capstone C30 es con una $\pi=3.2$ y TET $=1050{ }^{\circ} \mathrm{C}$. A partir del coeficiente de derrateo se observa que se tiene un decremento en la potencia del $5.5 \%$ por cada $500 \mathrm{msnm}$ y del $3.9 \%$ por cada $5^{\circ} \mathrm{C}$ que aumenta la temperatura promedio del medio ambiente.

Una vez que se ubica la turbina bajo condiciones de operación real, a una temperatura y presión determinadas, que en este caso son las de la Ciudad de México, se evaluó el efecto que se tiene en el coeficiente convectivo de transferencia de calor del lado de los gases en la caldera, con el suministro de los gases de combustión provenientes de la MTG para sustituir una fracción del aire requerido para la combustión y generación de vapor. El ahorro de energía determinado en este trabajo equivale al $10 \%$ del combustible suministrado de forma convencional, a este ahorro hay que sumarle los beneficios que se obtienen con el incremento del coeficiente convectivo de transferencia de calor, que con un bajo exceso de aire (10\%) se puede tener un $12 \%$ de incremento en ese coeficiente. Lo que demuestra ampliamente la ventaja adicional de emplear un sistema convencional de generación de vapor para la cogeneración.

\section{ACKNOWLEDGMENT}

The authors acknowledge the financial support received through the Mexican Center for Innovation in Solar Energy (CeMIE-Sol), as part of the Call 2013-02, the SECTOR FUND CONACYT-SENER-ENERGY SUSTAINABILITY within Strategic Project No. 10, entitled: "SOLAR FUELS AND INDUSTRIAL PROCESSES", the support scholarship by CONACYT-SENER with number 814358 and the collaboration with the agency Deutsche Gesellschaft für Internationale Zusammenarbeit GIZ México for the development of this work.

\section{REFERENCES}

[1] CONUEE, "Estudio sobre Cogeneración en el Sector Industrial en México", México D.F., 2009

[2] H.I. Onovwiona, V.I. Ugursal "Residential cogeneration systems: review of the current technology" Renewable and Sustainable Energy Reviews 2006, vol. 10, pp. 389-431.

[3] Llamas A., Probst O., "On the role of efficient cogeneration for meeting Mexico's clean energy goals" Energy Policy, 2018, 112, pag. 173-183

[4] Energy Nexus Group. "Technology characterization micro-turbines". USA: Environmental Protection Agency. 2002

[5] Murugan S., B. H. "A review of micro combined heat and power systems for residential applications". Renewable and Sustainable Energy Reviews, 2016 pp. 144-162.

[6] Capstone. "Capstone Turbine Corporation". Obtenido de https://www.capstoneturbine.com/, 2017

[7] SENER, "Balance nacional de energía 2014", Primera edición, México, 2015

[8] Cengel Y., Boles M. (2015), "Termodinámica", Mc Grawll Hill, octava edición.

[9] Backman J., Kaikko J., (2011), "Microturbine systems for small combined heat and power (CHP) applications", University of Technology, Finland.

[10] Arav B., Shulman R., (2016), "Basic concepts for forcing of lowpower micro turbine generators", Procedia Engineering, 150, pag. $1384-1390$.

[11] Visser W., Shakariyants A., (2011), "Development of a $3 \mathrm{~kW}$ Microturbine for CHP Applications", Engineering for Gas Turbines and Power, vol 133 
[12] Kesseli J., Wolf T., (2003), "Micro, industrial, and advanced gas turbines employing recuperators", Proceedings of ASME Turbo Expo 2003.

[13] Xiao G., Yang T., (2017), "Recuperators for micro gas turbines: A review", Applied Energy, 197, pag. 83-93.

[14] Van Wiley, "Fundamentos de termodinámica", Limusa Wiley, 2 edición, México, 2008.

[15] A. Santamaria, H. Romero-Paredes y N. Segura. "Análisis paramétrico y evaluación estadística de las turbinas de gas comerciales empleadas para cogeneración hasta 50MW". Revista de Investigación y Desarrollo 2016, vol. 2, pp. 82-91.

[16] Plauchú A., "Eficiencia en sistemas de generación y distribución de vapor", Edición 2006, México

[17] Rayaprolu K. "Boilers for power and process", CRC Press, United States of American, 2009.

[18] Cleaver Brooks, "Model CB, 15 - 100 HP Steam and Hot Water Dryback Integral Burner", http://www.cleaverbrooks.com/
[19] Rohsenow W., Hartnett J., "Handbook of heat transfer", Mc Graw Hill, 1973

[20] Afgan N., Schiünder E., "Heat Exchangers: Design and theory sourcebook", Mc Graw Hill, 1974.

[21] Kakac S., "Heat Exchangers, Selection, rating, and termal design", CRC Press, Thrid edition, 2012.

[22] Xiao G., Yang T., "Recuperators for micro gas turbines: A review", Applied Energy, 2017, 197, pag. 83-93

[23] Thu K., Baran B., "Thermodynamic analysis on the part-load perfomance of a microturbine system for micro/mini CHP applications", Applied Energy, 2016, 178, pag. 600-608

[24] Bracco S., Delfino F., "A mathematical model for the dynamic simulation of low size cogeneration gas turbines within Smar microgrids", Energy, 2017, 119, pag. 710-723. 


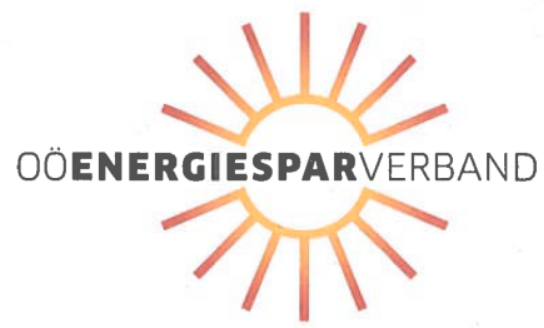

Estudiante de Posgrado de Energía y Medio Ambiente

Universidad Autónoma Metropolitana-Iztapalapa

Av. San Rafael Atlixco No. 186

09340 Col. Vicentina, Deleg. Iztapalapa

México, D. F

Wels, March 2019

\section{Confirmation of participation}

WORLD SUSTAINABLE ENERGY DAYS, 27 February - 1 March, Wels/Austria

of Ms. Adriana Santamaria Padilla

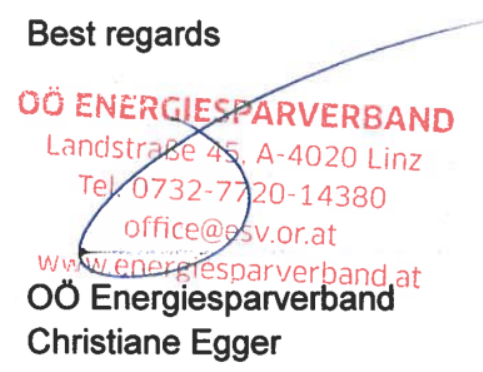

OÖ ENERGIESPARVERBAND, Landstraße 45, A-4020 Linz, Austria (Eingang: Martin-Luther-Platz 3) Tel. +43-732-7720-14380, office@esv.or.at, www.esv.or.at, UID: ATU 39283707, zVR 171568947 Partner der Netzwerke FEDARENE und EUFORES 


\title{
Heat recovery with steam boiler of waste heat from MGT: Thermodynamic analysis.
}

\begin{abstract}
Adriana Santamaria Padilla, Eng. in energy, Master's student Posgrado en Energía y Medio Ambiente. Universidad Autónoma Metropolitana-Iztapalapa. Av. San Rafael Atlixco No. 186, Col. Vicentina, Del. Iztapalapa, C.P. 09340, Ciudad de México, México. Cell phone: (+521) 5539868634, E-mail: aspenergia@xanum.uam.mx Website: https://www.researchgate.net/profile/Adriana_Santamaria_Padilla
\end{abstract}

Hernando Romero Paredes Rubio. PhD in energy, Research Professor. Departamento de Ingeniería de Procesos e Hidráulica. Universidad Autónoma Metropolitana-Iztapalapa. Posgrado en Energía y Medio Ambiente. Av. San Rafael Atlixco No. 186, Col. Vicentina, Del. Iztapalapa, C.P. 09340, Ciudad de México, México. Cell phone: (+521) 5554057986, E-mail: hrp@xanum.uam.mx Website: https://www.researchgate.net/profile/Hernando_Romero-Paredes_R. En período sabático en el Instituto de Energías Renovables, Universidad Nacional Autónoma de México.

\begin{abstract}
The objective of this paper is to demonstrate the advantages of using micro gas turbine (MGT) exhaust gasses to substitute the necessary air inside the boiler combustion chamber. The C65 and AE100 MGT performance was evaluated. Because the exhaust gasses from MGT have high excess air, they can be used to feed the necessary air inside the boiler burner. This work evaluates the use of those gasses on the heat convection transfer coefficient inside the boiler fluxes effect and the amount of energy saving. A thermodynamic analysis model was developed to know the boiler behavior when the exhaust gasses are used as air for the combustion process. It was gotten the operational parameters of the MGT C65 which are: pressure ratio $=3.7$ and the temperature turbine inlet $=1200^{\circ} \mathrm{C}$. Derating coefficient of C65 and AE100 turbine was evaluated and then for each 500 meters above sea level the generated power decrease 6.2 and $6.5 \%$ and when temperature increase $5^{\circ} \mathrm{C}$ power decrease 3.8 and $4.7 \%$ respectively. Meanwhile, when the exhaust gasses of the MGT are supplied to the boiler it could saving about of $10 \%$ of fuel due to the temperature. Because MGT exhaust gasses have less oxygen than air, the mass flow supplied to the boiler is increased and then the flow regime too. With these phenomena the convection heat transfer coefficient is higher than the conventional ones. It was found that this coefficient increased between $15-20 \%$ depending of the fuel used (NG or LPG) respect to the original system.
\end{abstract}

\section{Nomenclature}

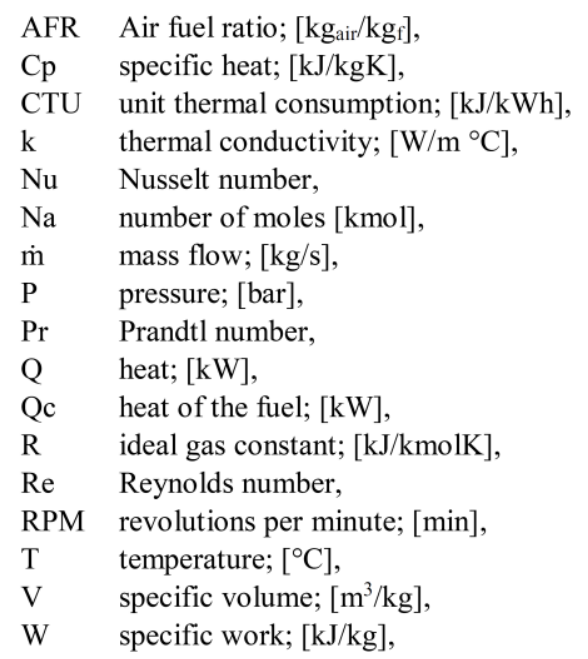

$\begin{array}{ll}\text { real } & \text { real, } \\ \text { sg } & \text { stack gases, } \\ \text { sic } & \text { isentropic compressor, } \\ \text { sit } & \text { isentropic turbine, } \\ \text { st } & \text { stoichiometric, } \\ \text { sum } & \text { supplied, } \\ \text { T } & \text { turbine, } \\ \text { tu } & \text { tube, } \\ \text { th } & \text { thermal, } \\ \text { u } & \text { universal, } \\ \text { Greek letters } \\ \pi & \text { compressor ratio, } \\ \eta & \text { efficiency; }[\%] \\ \rho & \text { density; }\left[\mathrm{kg} / \mathrm{m}^{3}\right] \\ \alpha & \text { mole of oxygen }[\mathrm{kmol}] \\ \mu & \text { viscosity }[\mathrm{kg} / \mathrm{ms}]\end{array}$



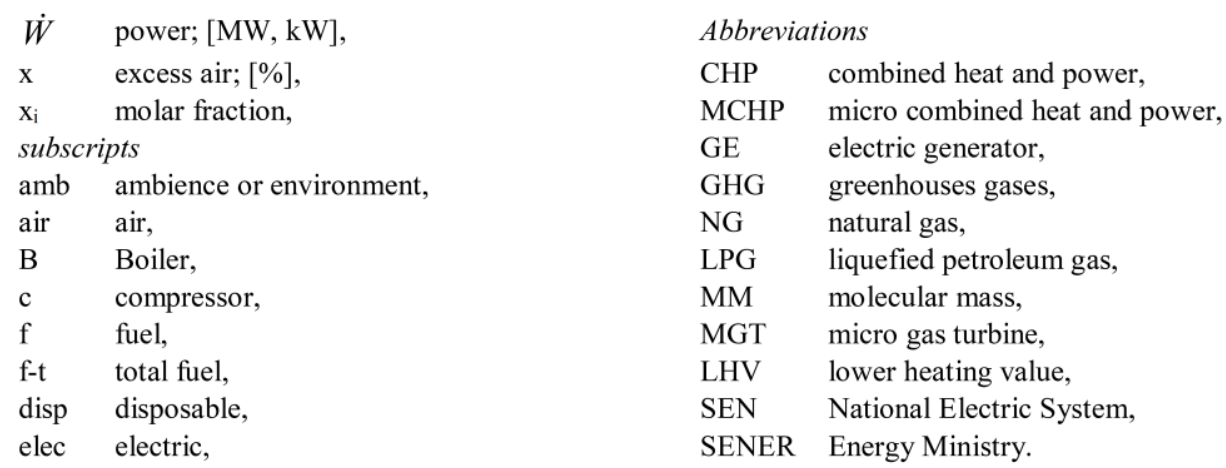

\section{Introduction}

Nowadays the ecosystems have been affected by contaminants emissions of all kinds of substances and unfortunately grow continuously. In the case of the atmosphere it is affected seriously due to the combustion of fossil fuels participating with a little more than $70 \%$ of the emissions that contributes substantially in the global warming. In order to reduce such affectations, it is important to make an efficient use of fuels, and, where appropriate, their replacement by renewable energies.

The CHP technologies are an option to increase the efficiency of the use of fossil fuels. In Mexico, the cogeneration, according to the article 36, fraction II, of the electrical industry law and their regulation article 103, understand as:

I. Power production together with steam or another secondary thermal energy, or both.

II. Direct or indirect power production from a thermal energy that doesn't use in the process.

III. Direct or indirect power production using fuels generated in the industrial process.

The CHP can have efficiencies as high as $80 \%$ [1]. Then, this is a good alternative to mitigate greenhouses gases (GHG) and promote energy sustainability. The main energy, economics and ecological benefits are:

- Decrease in primary energy consumption

- Decrease in fuel imports

- Reduction of GHG emissions

- Reduction of losses in the electrical system

- Increase in industrial competitiveness

- Motivation for the research and development of energy efficient systems

- Possibility to reduce up to 1000 tons of $\mathrm{CO}_{2}$ per $\mathrm{GWh}$ of power, depending of the sources heat and power displaced [2]

In Mexico the thermal energy used is around $77 \%$ of total energy consumption. By the way, the use of waste heat is not normally used in industries. The CHP can be lower or higher system. The lower system takes his advantage for the use of waste heat for power production. The distributed generation uses different technologies that are based on: steam turbine, gas turbine and reciprocating engine.

Figure 1 shows the historical evolution of the capacity installed of CHP in Mexico with data provided by Energy Regulatory Commission (CRE) into the period from 1994-2017. In 2015, many licenses for cogeneration were agreed. These are due to Mexican energy reform, which impulse this kind of technologies in order to get the goal of GHGs mitigation by the year 2030. By the year 2017 the installed capacity was almost 7,000 MW. According to SENER/CONUEE/GIZ report (2009), The total potential for CHP could be near of 10,000 MW [3]. 
Currently, the cogeneration represents around $9 \%$ of the world's electric generation and it's estimated that by 2030 there be a growth of $30 \%$ [4]. In the Figure 2 shows that there are five countries that have expanded successfully the use of cogeneration to around $30-50 \%$ of total power generation: Denmark, Finland, Russian, Latvia and Netherlands. Each of these countries has their own approach, but their collective experience demonstrates what can be achieved [5].

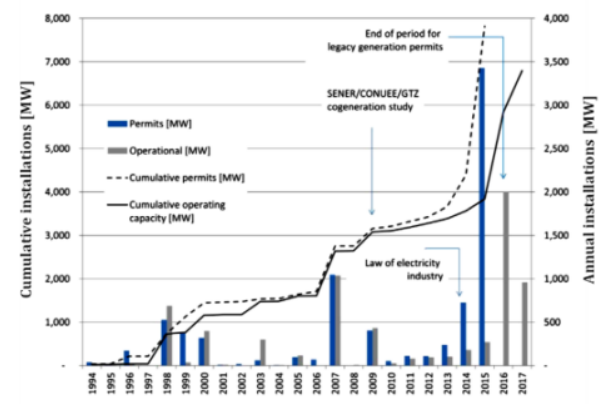

Figure 1.- Historical evolution of the capacity installed of CHP in Mexico (1994-2017).

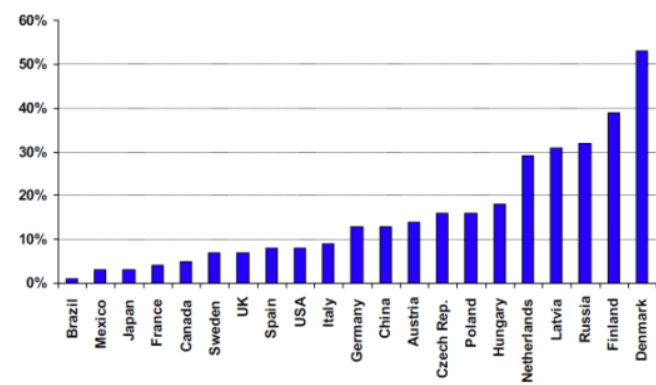

Figure 2.- CHP share of total national power production.

Because cogeneration produces both heat and electricity in the consumption center, it is possible the use of waste heat from micro gas turbine (MGT) for any heat application as: water heating, steam production, absorption refrigeration system, air conditioning, heating, between others [6]. The mayor industrial potential are: Food industry and beverages, pulp and paper, textiles, hospitals, hotels, administration buildings.

The micro-generation systems (MCHP) usually implies a primary motor: Stirling motor, alternative engines, MGT, Steam turbines, and fuel cells [7]. There exists a particular interest on the potential use of MTG and alternative engines for the small applications.

In this work, a feasible analysis to use MTG waste gases into the conventional steam boiler to substitute the combustion air and reduce the energy consumption is made. The analysis and an evaluation of the Capstone C65 and Ansaldo Energia AE100 MGT waste heat gases uses into the Cleaver Brooks 100 and 150 BHP (4WG) steam boilers is made under different operational conditions. The main objective is to determine the possible effects on the steam boiler operation with a higher gas flow (instead of air flow) and a higher temperature from MGT.

\section{Methodology}

\section{Micro gas turbines (MGT)}

The MGT combustion stack gases temperature is between 270 and $320^{\circ} \mathrm{C}$, as a function of generated power and atmospheric conditions. Frequently those gases are sent to the environment. In order to profit of the waste heat, it is important to do a thermodynamic analysis to know the feasibility of implementation the use of that gases into a conventional stem boiler and avoid investing in a heat recovery system.

Table 1 shows the MTG specifications provided by the manufacturers [8] [9].

Table 1.- Specifications of the MGTs.

\begin{tabular}{|l|c|c|}
\hline \multicolumn{1}{|c|}{} & Capstone C65 & Ansaldo Energia AE100 \\
\hline Electrical power output [kW] & 65 & 100 \\
\hline Electrical efficiency LHV [\%] & 29 & 30 \\
\hline Fuel inlet pressure [bar] & $5.17-5.52$ & $0.02-0.1$ \\
\hline Fuel consumption $[\mathrm{kW}]$ & 223 & 333 \\
\hline Exhaust gas flow $[\mathrm{kg} / \mathrm{s}]$ & 0.49 & 0.80 \\
\hline Exhaust gas temperature $\left[{ }^{\circ} \mathrm{C}\right]$ & 309 & 280 \\
\hline Thermal power available at $70^{\circ} \mathrm{C}[\mathrm{kW}]$ & 120 & 192 \\
\hline
\end{tabular}


The analysis will be performed using NG and LPG as fuel, with the composition shown in Table 2, obtaining a LHV of $48,813 \mathrm{~kJ} / \mathrm{kg}$ and $46,024 \mathrm{~kJ} / \mathrm{kg}$ respectively [10].

\begin{tabular}{|c|c|c|c|}
\hline \multicolumn{2}{|c|}{ NG } & \multicolumn{2}{|c|}{ LPG } \\
\hline Composition & Percent [\%] & Composition & Percent $[\%]$ \\
\hline $\mathrm{CH}_{4}$ & 94 & $\mathrm{C}_{3} \mathrm{H}_{8}$ & 30 \\
\hline $\mathrm{C}_{2} \mathrm{H}_{6}$ & 3 & $\mathrm{C}_{4} \mathrm{H}_{10}$ & 70 \\
\hline $\mathrm{CO}_{2}$ & 1 & & \\
\hline $\mathrm{N}_{2}$ & 1 & & \\
\hline $\mathrm{C}_{3} \mathrm{H}_{8}$ & 0.5 & & \\
\hline $\mathrm{C}_{4} \mathrm{H}_{10}$ & 0.3 & & \\
\hline $\mathrm{C}_{5} \mathrm{H}_{12}$ & 0.2 & & \\
\hline
\end{tabular}

The operating conditions are determined from the MGT manufacturer's data under ISO conditions. The fuel flow is calculated using (3):

$$
\begin{aligned}
E c & =\frac{\dot{W}}{\eta_{\text {elec }}} \\
E c & =\dot{m}_{f} L H V \\
\dot{m}_{f} & =\frac{E c}{L H V}
\end{aligned}
$$

The air flow is determined from the flow of the exhaust gases:

$$
\dot{m}_{\text {air }}=\dot{m}_{s g}-\dot{m}_{f}
$$

The air-fuel ratio $\left(A F R_{\text {real }}\right)$ is then calculated:

$$
A F R_{\text {real }}=\frac{\dot{m}_{\text {air }}}{\dot{m}_{f}}
$$

To obtain the air - fuel ratio stoichiometric $\left(A F R_{s t}\right)$, the combustion mass balance is used accordingly with the NG composition, as shown in Table 2, as:

$$
\mathrm{CnHm}+(1+x)\left(n+\frac{m}{4}\right)\left(\mathrm{O}_{2}+3.76 \mathrm{~N}_{2}\right) \rightarrow n \mathrm{CO}_{2}+\frac{m}{2} \mathrm{H}_{2} \mathrm{O}+x\left(n+\frac{m}{4}\right) \mathrm{O}_{2}+(1+x)\left(n+\frac{m}{4}\right) 3.76 \mathrm{~N}_{2}
$$

The following compact formula is obtained:

$$
C_{1.037} H_{4.034}
$$

Whose molecular mass is:

$$
M M=12 n+m
$$

The $A F R_{s t}$ is (9):

$$
A F R_{s t}=\frac{\left(n+\frac{m}{4}\right) * 4.76 * 29}{M M}
$$

The $A F R_{\text {real }}$ is then calculated as follows:

$$
A F R_{\text {real }}=(1+x)\left(A F R_{s t}\right)
$$

From the equation (10) the excess air is obtained: 


$$
x=\frac{A F R_{\text {real }}}{A F R_{s t}}-1
$$

Figure 3 shows the schematic operation diagram of the MGT. The first step is the air suction by the compressor. The air is compressed at a given pressure. Then pass through the heat recovery and enters to the combustion chamber where is mixed with fuel. These gases expand into the gas turbine producing work and finally pass through the heat recovery (recuperators). A fraction of the obtained work is used by the compressor and the other fraction is converted into electrical energy by means of the generator [11].

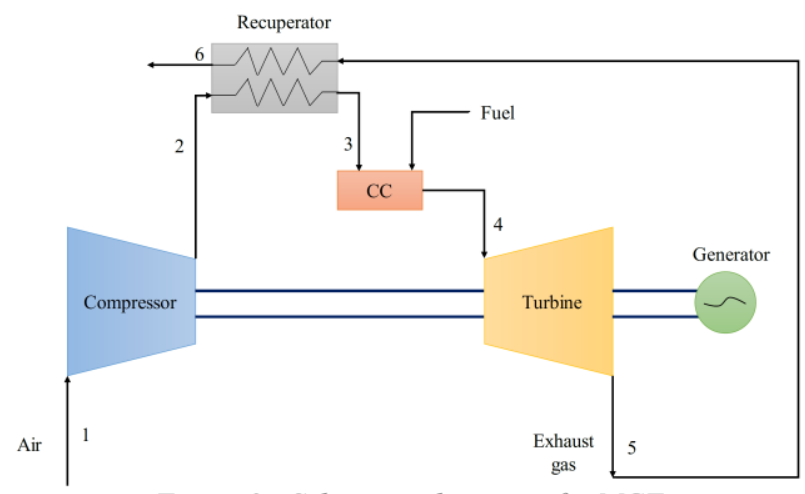

Figure 3.- Schematic diagram of a $M G T$

The thermodynamic properties of the study MGTs are determined as follows:

State 1.- Admission of air to the compressor. The following atmospheric conditions are taken as reference state: $\mathrm{T}=15^{\circ} \mathrm{C}$ and $\mathrm{P}=1.013$ bar. With these values and using the ideal gas equation, the specific volume is obtained considering one mole of air:

$$
v=\frac{R_{a i r} T}{P}
$$

To calculate the specific air volume of the MGT, the air volume at ISO conditions is used, which will remain constant in present analysis, since the turbine is designed for that specific volume regardless of atmospheric conditions. However, the mass flow does change:

$$
\dot{V}_{\text {air }}=v_{1} \dot{m}_{\text {air } I S O}
$$

State 2.- Compressor air outlet. The manufacturer doesn't provide the $\pi$, for that reason it has been determined by iterations. Agreed with literature, it can operate in a range of $\pi=1.5$ to 7 ; therefore $\pi$ was varied in that interval [12][13]. Once $\pi$ is obtained, the compressor air outlet pressure $\left(\mathrm{P}_{2}\right)$ is obtained, and from the efficiency of the compressor (it's considered in the first instance of 0.8$)$ its temperature $\left(T_{2}\right)$ is obtained:

$$
T_{2}=T_{1}\left[1+\frac{1}{\eta_{\text {sic }}}\left(\pi^{x}-1\right)\right]
$$

State 4.- Inlet of stack gases to the turbine. Because manufacturers don't provide the inlet temperature to the MGT, It has to be computed. To obtain it, firstly the temperature range in which these turbines operate was searched in the literature and then by means of iterations it was delimited, finding a range from 700 to $1300{ }^{\circ} \mathrm{C}$ $[14][15][16]$. Also, considering that there aren't pressure drops in the regenerator and in the combustion chamber, $\mathrm{P}_{4}=\mathrm{P}_{2}$, therefore are known $\mathrm{T}_{4}$ and $\mathrm{P}_{4}$, finally $\mathrm{v}_{4}$ is obtained using (12).

State $5 \mathrm{~s}$.- Isentropic turbine outlet. Considering the isentropic expansion of the turbine, $\mathrm{T}_{5 \mathrm{~s}}$ is obtained with the following equation: 


$$
T_{5 s}=T_{4}\left(\frac{1}{\pi^{x}}\right)
$$

State 5.- Polytropic state or real state turbine outlet. From the efficiency of the turbine is determined $\mathrm{T}_{5}$ :

$$
\eta_{s i t}=\frac{T_{4}-T_{5}}{T_{4}-T_{5 s}}
$$

State 3.- Inlet to the combustion chamber. The air temperature at the inlet of the combustion chamber is obtained from the definition of the efficiency of the regenerator:

$$
E=\frac{T_{3}-T_{2}}{T_{5}-T_{2}}
$$

State 6.- Regenerator outlet of flow hot water. The temperature $\mathrm{T}_{6}$, which exit from the regenerator to process, is obtained from a mass and energy balance, as shown below:

$$
\dot{m} C p\left(T_{5}-T_{2}\right)=\dot{m} C p\left(T_{5}-T_{6}\right)
$$

It can also be obtained from the effectiveness of the regenerator:

$$
T_{6}=T_{5}-E\left(T_{5}-T_{2}\right)
$$

Once the thermodynamic states are known, the available heat of the MGTs can be calculated:

$$
\dot{Q}_{\text {disp }}=\dot{m}_{s g} C p_{s g}\left(T_{5}-T_{\text {req }}\right)
$$

It's well known that in order to generate mechanical power from the combustion of a mixture of fuel and air under certain conditions of pressure and temperature, environmental conditions play an important role [12][17]. The derating coefficient is calculated by varying the pressure as a function of the high above sea level (from 0 to 3500 masl, with intervals of 500 masl) and the temperature (from 5 to $50^{\circ} \mathrm{C}$, with intervals of $5^{\circ} \mathrm{C}$ ) [18].

\section{Boiler}

Boilers are equipment designed to transfer heat produced by combustion, or by electricity, to a given fluid, generates and delivers steam in the quantity and quality required by the end user [16].

The pyro-tubular boilers are frequently used in most industrial plants, where hot gases flow through the interior of the tubes that are immersed in water inside a shell as shown in Figure 4. The maximum design operating pressures are at 15 bar. Capacity varies between 10 and $800 \mathrm{BHP}$, equivalent to steam production of 160 to $12,500 \mathrm{~kg} / \mathrm{h} \mathrm{[19].}$
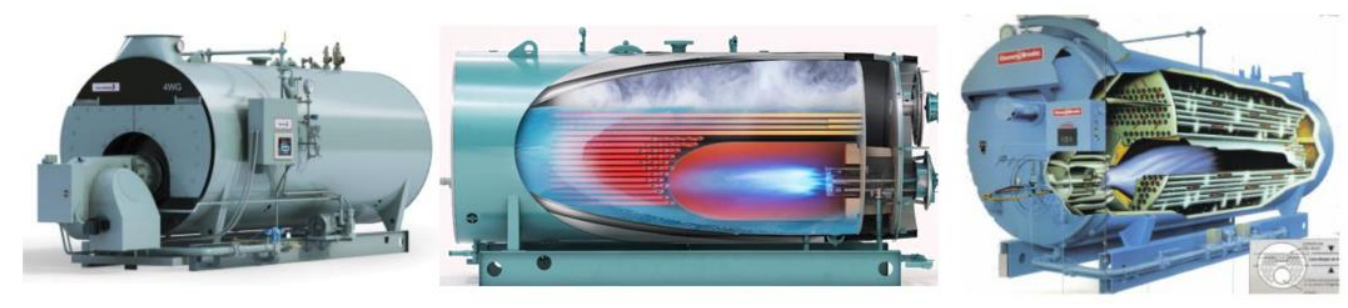

Figure 4.- Pyro-tubular boiler (fire tube) [20]

Figure 5 shows the schematic diagram of a 4-step Wetback boiler. The objective of this work is to replace the air flow with the gases coming from a MGT and thus take advantage of the heat of those gases to reduce the fuel consumption. Because these gases have high excess of air, in principle do not disturb the process of conventional combustion of the boiler. Under this premise, fuel consumption is reduced. It is therefore 
necessary to know the magnitude of the disturbance in the heat transfer coefficients between the tubes and water. Table 3 shows the boiler specifications of 100 and 150 BHP 4WG [21].

\begin{tabular}{|c|c|c|}
\hline BHP & $1004 W G$ & $1504 \mathrm{WG}$ \\
\hline Rated capacity $[\mathrm{kg} / \mathrm{h}]$ from and at $100^{\circ} \mathrm{C}$ & 1564.89 & 2347.34 \\
\hline Power output $[\mathrm{kW}]$ & 981.20 & 1471.51 \\
\hline Heating surface (Fireside) $\left[\mathrm{m}^{2}\right]$ & 46.45 & 69.68 \\
\hline Fuel flow $[\mathrm{kg} / \mathrm{s}]$ & 0.0245 & 0.0367 \\
\hline Tube diameter $[\mathrm{m}]$ & 0.0508 & 0.0508 \\
\hline Tube thickness [m] & 0.00279 & 0.00279 \\
\hline Length of the tubes [m] & 3.25 & 3.60 \\
\hline Number of tubes in the boiler & 85 & 115 \\
\hline
\end{tabular}

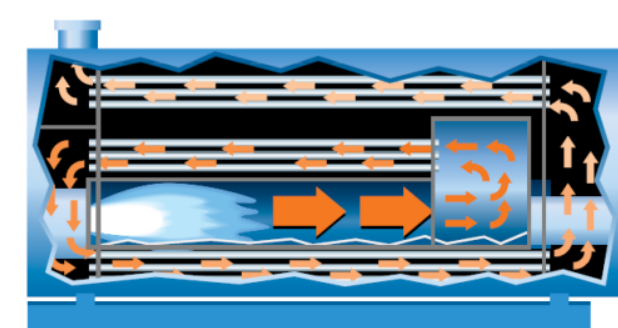

Figure 5.- Schematic diagram of boiler with 4 step Wet-Back [21]

To determine the convective heat transfer coefficient of the combustion gas side, the operating conditions of the boiler are analyzed under standard conditions [22][23]. The amount of standard steam available through the manufacturer's data is obtained:

$$
\dot{Q}_{\text {steam }}=\dot{m}_{\text {steamNom }} \Delta h
$$

Where $\Delta h$ is a function of the enthalpies of operating and environmental conditions, therefore, when defined to operate under new conditions, the actual steam flow is recalculated with the following equation:

$$
\dot{m}_{\text {steam Real }}=\frac{\dot{m}_{\text {steamNom }}}{f e}
$$

Where $f e$ is the evaporation factor.

The heat supplied by the fuel can be calculated:

$$
\dot{Q}_{\text {sum }}=\frac{\dot{Q}_{\text {steam }}}{\eta}
$$

Then the fuel flow is computed as:

$$
\dot{m}_{f}=\frac{\dot{Q}_{\text {sum }}}{L H V}
$$

Subsequently, the flow of combustion gases as a function of excess air, which varies from $0-25 \%$, is determined using (24).

$$
\dot{m}_{s g}=\left(A F R_{\text {real }}+1\right) \dot{m}_{f}
$$

The stoichiometric airflow of the boiler is obtained $(x=0)$ and with excess air $(x \neq 0)$.

$$
\dot{m}_{\text {air }}=\dot{m}_{s g}-\dot{m}_{f}
$$

Once these flows are known, the convective coefficient of the combustion gases can be calculated [21]: 
Using (25), the flue gas flow for each tube is obtained:

$$
\dot{m}_{s g, t u}=\frac{\dot{m}_{s g}}{N_{t u}}
$$

The velocity of the gases per tube is determined:

$$
v_{s g, t u}=\frac{\dot{m}_{s g, t u}}{\rho_{s g\left(\bar{T}_{s g}\right)} A_{t u}}
$$

Where the density of combustion gases is a function of their average temperature:

$$
\bar{T}_{s g}=\frac{T_{\text {Flame }}-T_{\text {prop }, s g}}{2}
$$

Once the velocity of combustion gases is known, the Reynolds is determined:

$$
\operatorname{Re}=\frac{v_{t u} d_{\text {int }}}{v}
$$

From the Reynolds number, correlations of the Nusselt number are used depending on the type of flow obtained (laminar, transient or turbulent). The desired convective coefficient is obtained with the Nusselt number.

$$
h_{1}=\frac{N u k}{D}
$$

Where the Nusselt is determined according to the flow regime [21]:

$$
\begin{aligned}
& \text { Turbulent flow: } N u=0.22 \operatorname{Re}^{0.8} \operatorname{Pr}^{0.5} \\
& \text { Transition flow: } N u=\frac{(f / 2)(\operatorname{Re}-1000) \operatorname{Pr}}{1+12.7 \sqrt{f / 2}\left(\operatorname{Pr}^{2 / 3}-1\right)}
\end{aligned}
$$

Where: $f=(1.58 \ln \operatorname{Re}-3.28)^{-2}$

\section{Coupling of stack gases MGT-Boiler}

In order to make the analysis of the coupling of the effluent gases from a MGT to a boiler, it is necessary to know its composition. It is obtained using (6) when this reaction is carried out with a certain amount of fuel moles $\left(\mathrm{Na}_{T}\right)$ and some excess air. With the data of the MTG the amount of fuel with which they are fed is known and the number of $N a_{T}$ fed can be calculated. Using (8) the molecular mass of the fuel is known and the number of $N a_{T}$ is determined:

$$
N a_{T}=\frac{\dot{m}_{f T}}{M M_{c}}
$$

Equation (6) can be rewritten to determine the composition of the exhaust gases:

$$
\mathrm{Na}_{T} \mathrm{C}_{n} \mathrm{H}_{m}+\left(1+x_{T}\right)\left[N a_{T}\left(n+\frac{m}{4}\right)\left(\mathrm{O}_{2}+3.76 \mathrm{~N}_{2}\right)\right] \rightarrow \mathrm{Na}_{T} n \mathrm{CO}_{2}+\mathrm{Na}_{T} \frac{m}{2} \mathrm{H}_{2} \mathrm{O}+x_{T} \mathrm{Na}_{T}\left(n+\frac{m}{4}\right) O_{2}+\left(1+x_{T}\right)\left[N a_{T}\left(n+\frac{m}{4}\right)\right] 3.76 N_{2}
$$

Once the composition of the exhaust gases and their flow is known, the available heat can be calculated using (20) and considering the value of Treq $=25^{\circ} \mathrm{C}$. With the amount of heat by the combustion gases and the required heat of the boiler using (23) the new fuel flow required by the boiler is calculated:

$$
\dot{Q}_{f 2}=\dot{Q}_{s u m}-\dot{Q}_{s g T}
$$




$$
\dot{m}_{f 2}=\frac{\dot{Q}_{f 2}}{L H V}
$$

With this flow of fuel for the boiler, the stoichiometric amount of oxygen that it requires is calculated:

$$
N a_{B} C_{n} H_{m}+\left[N a_{B}\left(n+\frac{m}{4}\right)\right]\left(O_{2}+3.76 N_{2}\right) \rightarrow N a_{B} n C O_{2}+N a_{B} \frac{m}{2} H_{2} O+\left[N a_{B}\left(n+\frac{m}{4}\right)\right] 3.76 N_{2}
$$

Using the composition of the gases coming from the MTG, the mass balance of the combustion is made, in order to know if there exist excess or deficiency of oxygen in those gasses:

$$
\begin{aligned}
& \mathrm{Na}_{B} \mathrm{C}_{n} \mathrm{H}_{m}+\mathrm{Na}_{T} n \mathrm{CO}_{2}+\mathrm{Na}_{T} \frac{m}{2} \mathrm{H}_{2} \mathrm{O}+x_{T} \mathrm{Na}_{T}\left(n+\frac{m}{4}\right) \mathrm{O}_{2}+\left(1+x_{T}\right)\left[\mathrm{Na}_{B}\left(n+\frac{m}{4}\right)\right] 3.76 \mathrm{~N}_{2} \rightarrow \\
& \mathrm{Na}_{T} n \mathrm{CO}_{2}+\mathrm{Na}_{B} n \mathrm{CO}_{2}+\mathrm{Na}_{T} \frac{m}{2} \mathrm{H}_{2} \mathrm{O}+\mathrm{Na}_{B} \frac{m}{2} \mathrm{H}_{2} \mathrm{O}+\alpha \mathrm{O}_{2}+\left(1+x_{T}\right)\left[\mathrm{Na}_{T}\left(n+\frac{m}{4}\right)\right] 3.76 \mathrm{~N}_{2}
\end{aligned}
$$

Where: $\quad \alpha<0$ it has oxygen deficit

$$
\begin{aligned}
& \alpha=0 \text { It is stoichiometric } \\
& \alpha>0 \text { it has excess oxygen }
\end{aligned}
$$

Taking a value of $\alpha<0$ the combustion equation is:

$$
\begin{aligned}
& \mathrm{Na}_{B} \mathrm{C}_{n} \mathrm{H}_{m}+\mathrm{Na}_{T} n \mathrm{CO}_{2}+\mathrm{Na}_{T} \frac{m}{2} \mathrm{H}_{2} \mathrm{O}+x_{T} \mathrm{Na}_{T}\left(n+\frac{m}{4}\right) \mathrm{O}_{2}+\left(1+x_{T}\right)\left[\mathrm{Na}_{B}\left(n+\frac{m}{4}\right)\right] 3.76 \mathrm{~N}_{2}+\left(1+x_{B}\right)\left[\alpha\left(\mathrm{O}_{2}+3.76 \mathrm{~N}_{2}\right)\right] \rightarrow \\
& \mathrm{Na}_{T} n \mathrm{CO}_{2}+\mathrm{Na}_{B} n \mathrm{CO}_{2}+\mathrm{Na}_{T} \frac{m}{2} \mathrm{H}_{2} \mathrm{O}+\mathrm{Na}_{B} \frac{m}{2} \mathrm{H}_{2} \mathrm{O}+x_{B} \alpha \mathrm{O}_{2}+\left(1+x_{T}\right)\left[\mathrm{Na}_{T}\left(n+\frac{m}{4}\right)\right] 3.76 \mathrm{~N}_{2}+\left(1+x_{B}\right) \alpha 3.76 \mathrm{~N}_{2}
\end{aligned}
$$

Equation (40) is used to determine the combustion gases flow as a function of the excess air into the boiler. With these flows, Reynolds number, Nusselt number and the convective heat transfer coefficient on the gas side are determined. In order to compute those parameters it is necessary to know the thermo-physical properties ( $\mu, \mathrm{k}, \mathrm{Cp}$ and $\mathrm{Pr}$ ) of gasses for both cases (conventional boiler operation and with MGT stack gasses).

The empirical Wilke equation (41) is used to get the $\mu$, the thermal conductivity is computed by Eucken equation (42) and the $\mathrm{Cp}$ and $\mathrm{Pr}$ are computed with equations 43 and 44 respectively. [24]:

$$
\begin{array}{ll}
\mu_{\text {mix }}=\sum_{i=1}^{n} \frac{x_{i} \mu_{i}}{\sum_{j=1}^{n} x_{j} \phi_{i j}} & \text { (41) } k_{\text {mix }}=\sum_{i=1}^{n} \frac{x_{i} k_{i}}{\sum_{j=1}^{n} x_{j} \phi_{i j}} \\
\bar{C} p_{\text {mix }}=\sum_{i=1}^{n} \bar{C} p_{i} \frac{x_{i}}{M M_{i}} & \text { (43) } \operatorname{Pr}=\frac{\bar{C} p \mu}{k}
\end{array}
$$

\section{Results}

\section{MGT analysis.}

Since $\pi$ and the inlet temperature of the gases to the turbine (TIT) are unknown, the iteration of 3 variables was performed: motor work, TIT and the pressure ratio. From this evaluation it was obtained that the MGT C65 operates with a $\pi=3.7$ and TIT $=1200^{\circ} \mathrm{C}$. Under these conditions, the power reported by the manufacturer and the heat available for the process reported in the literature are obtained [16] [25].

Known $\pi$ and TIT, the derating coefficient is obtained (Figure 6). It is observed that the behavior of the MGT C65 varying the atmospheric conditions (T, P), has a decrease in the power of $6.2 \%$ for every 500 masl and of $3.8 \%$ for every $5{ }^{\circ} \mathrm{C}$ that the temperature increases. 
In the case of the MGT AE100 the decrease in power is $6.5 \%$ for every 500 masl and $4.7 \%$ for every $5{ }^{\circ} \mathrm{C}$ that increases the temperature (Figure 7).

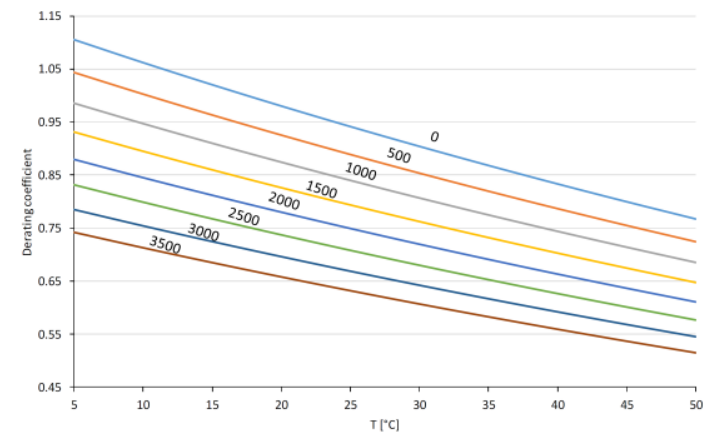

Figure 6.- Derating coefficient of MGT C65.

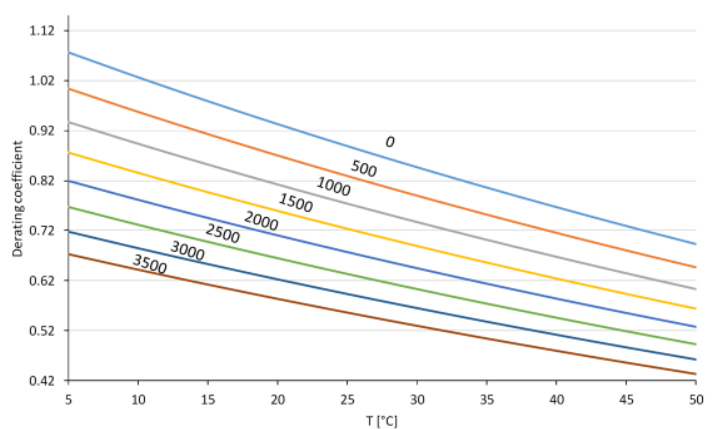

Figure 7.- Derating coefficient of MGT AE100

\section{Coupling of stack gases MGT-Boiler}

Once the variation of gas flow as a function of excess air and the fuel used in the conventional boiler operating or using stack gasses from the MGT are known (Figure 8 y Figure 9). It can be seen that the flow of gases from the MGT is greater than that conventionally used by the boiler. This situation can be negative if the operation regime is kept laminar because the internal global convective heat transfer coefficient, in the limit case, would remain constant and therefore the temperature of the gases in the boiler chimney would increase. Regarding the fuel used, it is observed that there is a greater flow of combustion gases using NG, this is because the $\mathrm{MM}_{\mathrm{LPG}}>\mathrm{MM}_{\mathrm{NG}}$, which implies that the number of moles of fuel required by the boiler is $\mathrm{Na}_{\mathrm{NG}}>\mathrm{Na}_{\mathrm{LPG}}$.

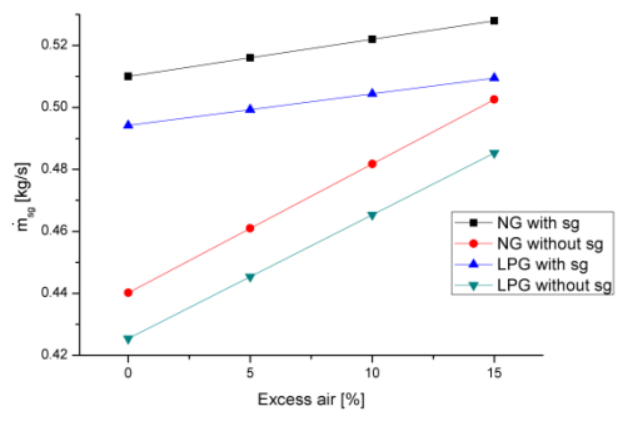

Figure 8.- Flow stack gases of MGT C65 as function of excess air

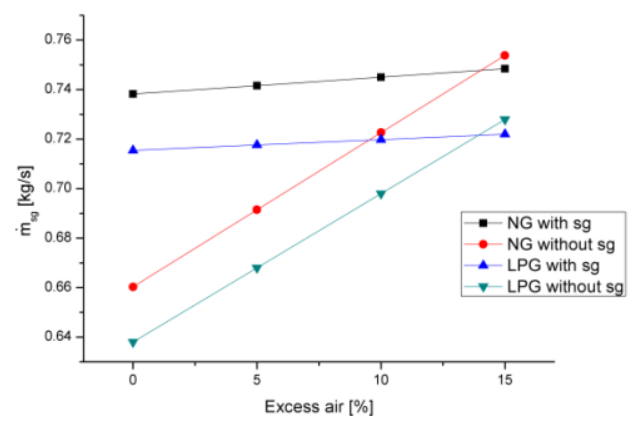

Figure 9.- Flow stack gases of MGT AE100 as function of excess air

The internal global convective heat transfer coefficient is a function of $\mathrm{Nu}, \mu, \mathrm{Cp}$ and $\mathrm{k} . \mu$ and $\mathrm{Cp}$ are directly function of the temperature. Then, because the temperature is inversely proportional to the excess air, the convective coefficient depends on the slope of $\mathrm{k}$ and $\mathrm{Nu}$ and it could be positive or negative. If the slope of $\mathrm{Nu}$ $>>\mathrm{k}$ the convective coefficient slope will be positive and inversely.

In order to corroborate if the behavior of the boiler is affected when using the gases coming from the MGT, the convective coefficient of heat transfer for both cases is evaluated (Figure 10 y Figure 11). It is observed that the convective coefficient is higher when the effluent gases of the MGT are supplied. However, the difference decreases as the excess air increases. It should be noted that a boiler that operates with NG and LPG hardly have an excess of air greater than $20 \%$. In this way the convenience of the use of the combustion gases of the MGT in a conventional boiler is demonstrated. 
It is also observed that in the presented cases, the thermal conductivity is greater than $\mathrm{Nu}$ slopes. Then the convective coefficient as a function of the excess air slope is negative when MGT stack gasses are used in the boiler.

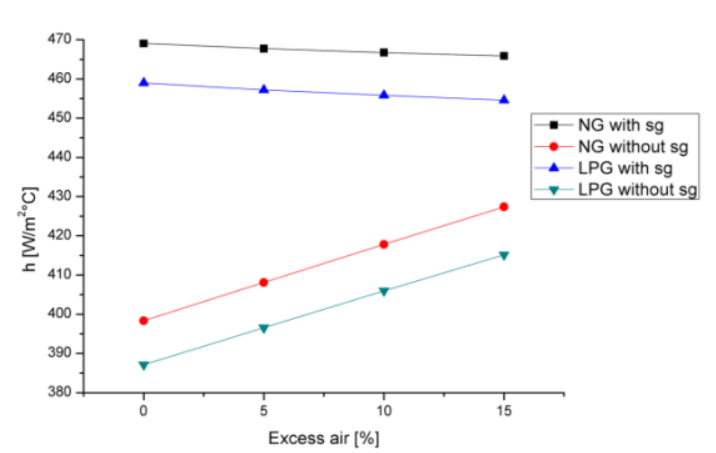

Figure 10.-Convective coefficient of MGT C65 as function of excess air

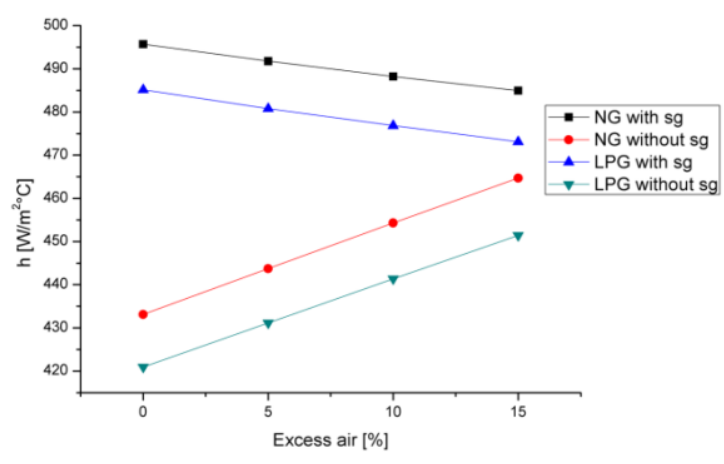

Figure 11.- Convective coefficient of MGT AE100 as function of excess air

\section{Conclusions}

In this work it was found that the operating conditions of the MGT of Capstone C65 is with a $\pi=3.7$ and TIT $=1200{ }^{\circ} \mathrm{C}$. From the derating coefficient it is observed that power decrease $6.2 \%$ for each 500 masl and of $3.8 \%$ for each $5{ }^{\circ} \mathrm{C}$ that increases the average environment temperature. In the case of the MGT AE100 it was found that power decrease $6.5 \%$ for every 500 masl and $4.7 \%$ for each $5{ }^{\circ} \mathrm{C}$ that increases the environment temperature.

Once the turbine is located under real operating conditions, at a certain temperature and pressure, which in this case are those of Mexico City, the effect of the convective heat transfer coefficient on the water side was evaluated. Gases in the boiler, with the supply of combustion gases from the MGT to replace a fraction of the air required for combustion and steam generation. The energy saving determined in this work is equivalent to $10 \%$ of the fuel supplied compared to conventional operation. To this energy saving we must add the benefits obtained with the increase of the convective heat transfer coefficient: with a low excess air (10\%) it can increase between $15-20 \%$ in that coefficient, depending on the fuel used (NG or LPG). The additional advantages have been amply demonstrated when using a conventional steam generator in MCHP systems.

\section{Acknowledgment}

The authors acknowledge the partial financial support received through the Mexican Center for Innovation in Solar Energy (CeMIE-Sol), as part of the Call 2013-02, the SECTOR FUND CONACYT-SENER-ENERGY SUSTAINABILITY within Strategic Project No. 10, entitled: "SOLAR FUELS AND INDUSTRIAL PROCESSES", and the support scholarship by CONACYT-SENER with number 814358.

\section{References}

[1] H. I. Onovwiona and V. I. Ugursal, "Residential cogeneration systems: review of the current technology," Renew. Sustain. Energy Rev., vol. 10, no. 5, pp. 389-431, Oct. 2006.

[2] P. A. Pilavachi, "Mini- and micro-gas turbines for combined heat and power," Appl. Therm. Eng., vol. 22, no. 18, pp. 2003-2014, 2002.

[3] CONUEE, CRE, and GIZ México, "Estudio sobre Cogeneración en el Sector Industrial en México," Conuee, Com. Nac. Para el Uso Efic. la Energ., pp. 1-156, 2009. 
[4] Agency International Energy, "Combined heat and power," 2008.

[5] M. M. Maghanki, B. Ghobadian, G. Najafi, and R. J. Galogah, "Micro combined heat and power (MCHP) technologies and applications," Renew. Sustain. Energy Rev., vol. 28, pp. 510-524, Dec. 2013.

[6] E. P. Agency, C. Protection, P. Division, and E. N. Group, "Technology Characterization: Microturbines Prepared for :," no. March, 2002.

[7] S. Murugan and B. Horák, "A review of micro combined heat and power systems for residential applications," Renew. Sustain. Energy Rev., vol. 64, pp. 144-162, Oct. 2016.

[8] Capstone, “C65 \& C65-ICHP MicroTurbine Natural Gas,” Power, no. 4, pp. 9-10, 2017.

[9] Ansaldo Energia, "Micro gas turbines AE T100 Micro gas turbines AE T100,” p. 10, 2016.

[10] SENER, "Lista de combustibles 2018 que se considerarán para identificar a los usuarios con un patrón de alto consumo, así como los factores para determinar las equivalencias en términos de barriles equivalentes de petróleo," 2018.

[11] Y. A. Çengel and M. A. Boles, Termodinámica (8a. ed.). 2015.

[12] J. L. H. Backman, "Microturbine systems for small combined heat and power (CHP) applications 7," vol. 1, 2011.

[13] B. Arav, R. Shulman, and V. Dooun, "Basic Concepts for Forcing of Low-Power Micro Turbine Generators," Procedia Eng., vol. 150, pp. 1384-1390, 2016.

[14] W. P. J. Visser, "Development of a 3 kW Microturbine for CHP Applications," vol. 133, no. April, 2011.

[15] J. Kesseli, J. Nash, T. Wolf, and S. Freedman, "MICRO, INDUSTRIAL, AND ADVANCED GAS TURBINES EMPLOYING RECUPERATORS," Proc. ASME Turbo Expo 2003, pp. 1-6, 2016.

[16] G. Xiao et al., "Recuperators for micro gas turbines : A review," Appl. Energy, vol. 197, pp. 83-99, 2017.

[17] J. Wiley, Fundamentals of momentun, heat, and mass transfer, 5th Editio. 2008.

[18] A. Santamaria and H. Romero-Paredes R., "Comparative analysis and evaluation of reciprocating engines and gas turbines used in cogeneration systems up to $50 \mathrm{MW}$," in 2017 IEEE 37th Central America and Panama Convention (CONCAPAN XXXVII), 2017, pp. 1-7.

[19] K. Rayaprolu, Boiler for Power and Process. 2009.

[20] Cleaver Brooks, "4WG - ICB Steam \& Hot Water 100-800 HP WET-BACK/DRY BACK FIRETUBES,” 2015.

[21] Cleaver Brooks, Boiler Book 2011. 2011.

[22] N. Afgan and F. Schiünder, Heat Exchangers: Design and theory sourcebook. 1974.

[23] W. M. Rohsenow and J. R. Hartnett, HANDBOOK OF HEAT TRANSFER, Third edit. 1998.

[24] R. B. Bird, W. E. Stewart, and E. N. Lightfoot, Fenômenos de Transporte, Segunda Edição. 2004.

[25] K. Thu, B. B. Saha, K. J. Chua, and T. D. Bui, "Thermodynamic analysis on the part-load performance of a microturbine system for micro/mini-CHP applications," Appl. Energy, vol. 178, pp. 600-608, 2016. 